University of Massachusetts - Amherst ScholarWorks@UMass Amherst

$5-1-2012$

\title{
Probing Novel Properties of Nucleons and Nuclei via Parity Violating Electron Scattering
}

Luis Rafael Mercado

University of Massachusetts - Amherst, 1rmercad@astro.umass.edu

Follow this and additional works at: http://scholarworks.umass.edu/open_access_dissertations

Part of the Physics Commons

\section{Recommended Citation}

Mercado, Luis Rafael, "Probing Novel Properties of Nucleons and Nuclei via Parity Violating Electron Scattering" (2012). Open Access Dissertations. Paper 587. 


\title{
PROBING NOVEL PROPERTIES OF NUCLEONS AND NUCLEI VIA PARITY VIOLATING ELECTRON SCATTERING
}

\author{
A Dissertation Presented \\ by \\ LUIS RAFAEL MERCADO
}

Submitted to the Graduate School of the

University of Massachusetts Amherst in partial fulfillment of the requirements for the degree of

DOCTOR OF PHILOSOPHY

May 2012

Physics Program 
(C) Copyright by Luis Rafael Mercado 2012

All rights reserved 


\title{
PROBING NOVEL PROPERTIES OF NUCLEONS AND NUCLEI VIA PARITY VIOLATING ELECTRON SCATTERING
}

\author{
A Dissertation Presented \\ by \\ LUIS RAFAEL MERCADO
}

Approved as to style and content by:

Krishna Kumar, Chair

Lorenzo Sorbo, Member

David Kawall, Member

Todd Tripp, Member

Donald Candela, Department Head

Physics Department 
To all those who believed in me and supported me through the years...

Specially to my mother for sacrificing so much to make sure I had a good education. 


\section{ACKNowledGEMEnts}

I would like to express my gratitude to everyone that has made this twelve year journey possible. My studies began at Indiana University with a concentration in Astrophysics and Mathematics. There, several professors suggested that I try to do research as part of an REU program. For the next two years, I had the pleasure of working with R. Kent Honeycutt and Constantine Deliyannis at Indiana University and Edward Churchwell at the University of Wisconsin.

After graduating, I was able to attend graduate school for Astrophysics at the University of Massachusetts thanks to a two-year fellowship from the NEAGEP, a group dedicated to increasing the diversity amongst science professors. During that time, my interests moved from understanding astronomical objects to how elementary particles interact to create matter.

I was introduced to the field of elementary physics by the person who would later become my research advisor. Many thanks go to Krishna Kumar for guiding me and making all this possible.

I met many other great individuals during the time I spent at Jefferson Labs that I would like to thank. These include Bryan Moffit, Jack Segal, Ed Folts, Alexandre Camsonne, Douglas Higinbotham, and Kees de Jager. A special thanks goes out to the members of the collaboration who helped train me and worked with me throughout the many stages of the preparations for HAPPEx/PREx. These people are Robert Micheals, Kent Paschke, Mark Dalton and Dustin McNulty. Last but not least, thanks and congratulations to the rest of the graduate students that worked many long shifts and late nights; Rupesh Silwal, Abdurahim Rakhman, Megan Friend, Kiadtisak Saenboonruang, Zafar Ahmed and Chun-Min Jen 


\begin{abstract}
PROBING NOVEL PROPERTIES OF NUCLEONS AND NUCLEI VIA PARITY VIOLATING ELECTRON SCATTERING
\end{abstract}

\author{
MAY 2012 \\ LUIS RAFAEL MERCADO, B.S., INDIANA UNIVERSITY BLOOMINGTON \\ PH.D., UNIVERSITY OF MASSACHUSETTS AMHERST
}

Directed by Krishna Kumar

This thesis reports on two experiments conducted by the HAPPEx (Hall A Proton Parity Experiment) collaboration at the Thomas Jefferson National Accelerator Facility. For both, the weak neutral current interaction (WNC, mediated by the $Z^{0}$ boson) is used to probe novel properties of hadronic targets. The WNC interaction amplitude is extracted by measuring the parity-violating asymmetry in the elastic scattering of longitudinally polarized electrons off unpolarized target hadrons. HAPPEx-III, conducted in the Fall of 2009, used a liquid hydrogen target at a momentum transfer of $Q^{2}=0.62 \mathrm{GeV}^{2}$. The measured asymmetry was used to set new constraints on the contribution of strange quark form factors $\left(G_{E, M}^{s}\right)$ to the nucleon electromagnetic form factors. A value of $A_{P V}=-23.803 \pm 0.778$ (stat) \pm 0.359 (syst) ppm resulted in $G_{E}^{s}+0.517 G_{M}^{s}=0.003 \pm 0.010$ (stat) \pm 0.004 (syst) \pm 0.009 (FF).

PREx, conducted in the Spring of 2010, used a polarized electron beam on a ${ }^{208} \mathrm{~Pb}$ target at a momentum transfer of $Q^{2}=0.009 \mathrm{GeV}^{2}$. This parity-violating asymmetry can be used to obtain a clean measurement of the root-mean-square radius of the neutrons in the ${ }^{208} \mathrm{~Pb}$ nucleus. The $Z^{0}$ boson couples mainly to neutrons; the neutron weak charge is much larger than that of the proton. The value of this asymmetry is at the sub-ppm level and has a projected experimental fractional precision of $3 \%$.

We will describe the accelerator setup used to set controls on helicity-correlated beam asymmetries and the analysis methods for finding the raw asymmetry for HAPPEx-III. We will also discuss in some detail the preparations to meet the experimental challenges associated with measuring such a small asymmetry with the degree of precision required for PREx. 


\section{TABle of Contents}

ABSTRACT vi

LIST OF TABLES. vii

LIST OF FIGURES xii

\section{CHAPTERS}

1 HISTORICAL BACKGROUND AND PHYSICS MOTIVATION

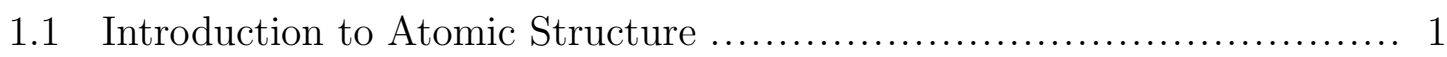

1.2 Modeling the Atom and its Components ............................. 2

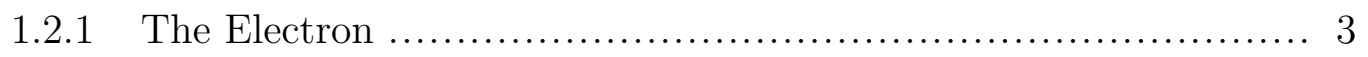

1.2.2 The Nucleus ............................................. 4

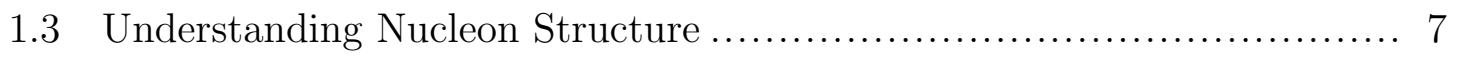

1.4 The Weak Interaction and Parity Violation............................. 10

1.5 Strangeness in Nucleons............................................. 12

1.6 Using Parity Violation to Probe the Nucleon.............................. 13

1.6.1 Electromagnetic Amplitude for Electron-Proton Scattering........ 14

1.6.2 Weak Neutral Interaction Amplitude ............................. 16

1.6.3 The HAPPEx-III Measurement .............................. 18

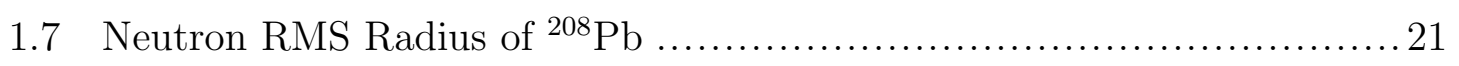

2 EXPERIMENTAL DESIGN ............................................ 26

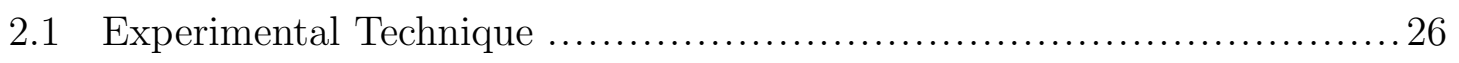

2.1.1 Interpreting our Asymmetry Measurement .......................28 
2.1.2 False Asymmetries and Other Corrections........................29

2.1.3 Backgrounds ............................................... 30

2.2 Polarized Beam....................................................... 30

2.2.1 Pockels Cell ..................................................... 32

2.2.2 Insertable Half-Wave Plate ..................................... 32

2.2.3 GaAs Photocathode ............................................... 32

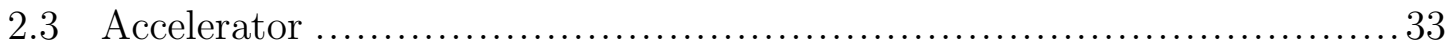

2.4 Beamline Components .................................................. 35

2.4.1 Current Monitors.................................................. 35

2.4.2 Position Monitors ................................................ 36

2.4.3 Modulation Coils ................................................. 37

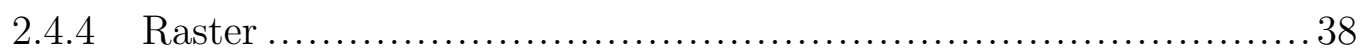

2.5 Target Ladder ........................................................ 39

2.6 Hall A High Resolution Spectrometers ................................ 40

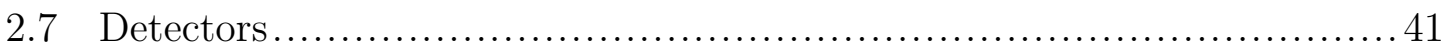

2.7.1 Standard Hall A Detector Package ................................ 42

2.7.2 HAPPEx Detector .......................................... 43

2.8 Data Acquisition ..................................................... 45

2.8.1 Standard DAQ .............................................. 45

2.8.2 Integrating Mode................................................ 45

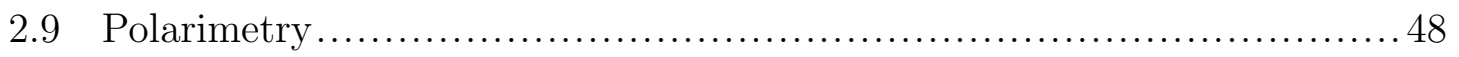

2.9.1 Moller Polarimeter ............................................... 49

2.9.2 Compton Polarimeter ............................................ 49

2.10 Luminosity Monitor ................................................ 52

3 PREX PREPARATIONS................................................. 54

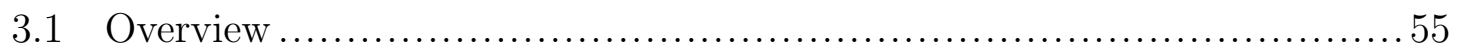

3.2 Double Wien Filter .................................................. 56 


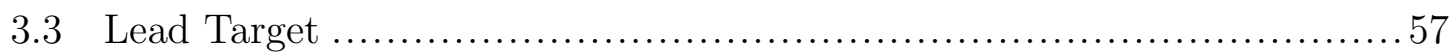

3.3.1 Target Stability $\ldots \ldots \ldots \ldots \ldots \ldots \ldots \ldots \ldots \ldots \ldots \ldots \ldots \ldots \ldots \ldots \ldots \ldots . \ldots \ldots$

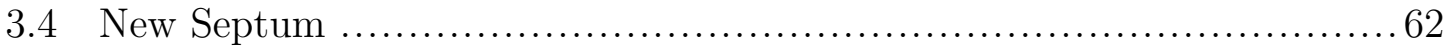

3.5 PREx Detector Package............................................. 63

3.6 Higher Resolution Analog to Digital Converters.......................... 65

3.6.1 Pedestals ..................................................... 67

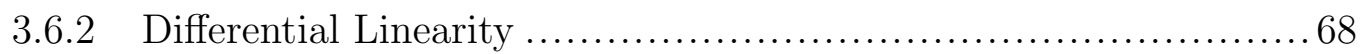

3.6.3 Cross-Talk .................................................... 70

3.7 Detector Linearity ............................................... 71

3.7.1 Experimental Test Setup ...................................... 72

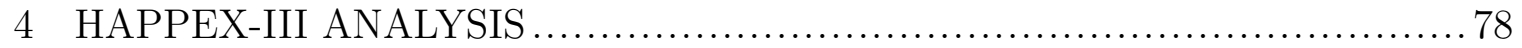

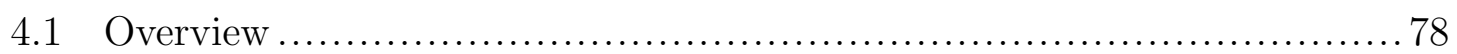

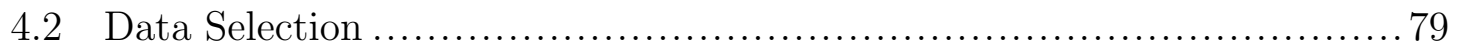

4.3 Raw Asymmetry .................................................. 83

4.4 False Asymmetry Corrections . ....................................... 84

4.4.1 Beam Modulation Analysis ...................................... 88

$4.5 \quad Q^{2}$ Determination............................................... 91

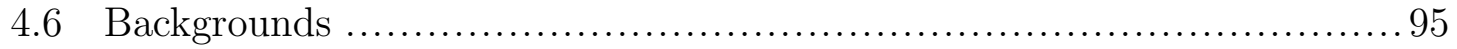

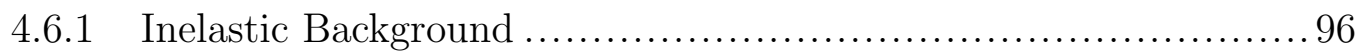

4.6.2 Scattering off target end caps ................................. 99

4.7 Finite Acceptance.......................................................... 99

4.8 Beam Polarization .................................................... 101

4.9 Final Physics Asymmetry ............................................ 104

5 RESULTS AND CONCLUSIONS ........................................... 105

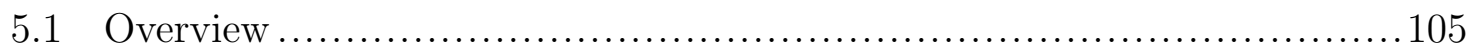

5.2 Determining Strange Quark Contribution .............................. 105

5.3 Summary of Past Results ......................................... 107 


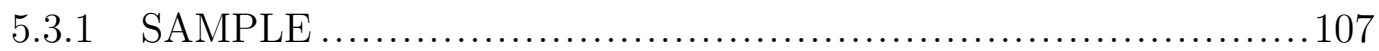

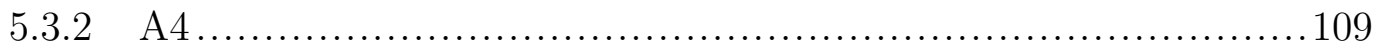

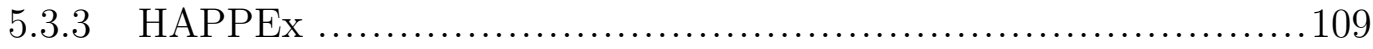

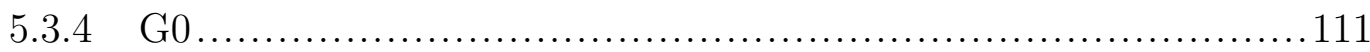

5.3.5 Summary of Measurements at $Q^{2} \approx 0.1 \mathrm{GeV}^{2} \ldots \ldots \ldots \ldots \ldots \ldots . \ldots 113$

5.4 Other Applications of PVES Results .................................. 114

5.5 Implications of HAPPEx-III Result ............................... 117

5.6 The Future of Parity Violation Expriments ............................. 119

5.6.1 A4-III Results and Developments ............................119

5.6.2 PREx-II .................................................... 119

5.6.3 $12 \mathrm{GeV}$ MOLLER .................................................. 120

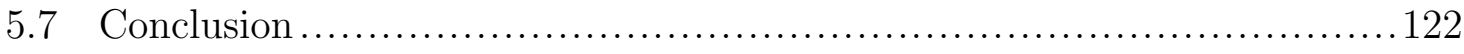

\section{APPENDICES}

A LINEARITY OF PHOTOMULTIPLIER TUBES …........................ 123

B STUDYING NOISE CONTRIBUTIONS WITH LUMIS ........................128

C ENSURING QUALITY OF DATA WITH SYNC MONITOR .................... 137

D MINIMIZING BOILING EFFECTS WITH CRYOTARGETS .................. 145

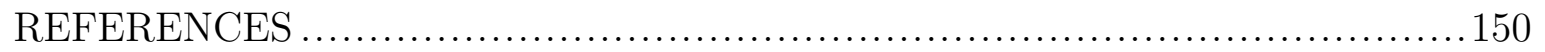




\section{LIST OF TABLES}

Table Page

1.1 Weak charges of leptons and quarks. 16

1.2 Summary of proposed kinematic conditions for HAPPEx-III. 18

2.1 Details about targets installed during the HAPPEx-III experiment. More details about the target configuration can be found in [22].

3.1 Summary of proposed experimental conditions and kinematics. .55

3.2 Pedestal Noise for the lowest gain levels with no signal connected. The value given in the last column is the pedestal difference divided by twice the ideal signal strength, $200 \mathrm{~K}$.

4.1 Summary of threshold cuts and intervals used to remove unwanted data. Extent values refer to the number of windows that have been cut from the data before (lo) and after (hi) an unwanted event. ..........8 80

4.2 Helicity Correlated charge asymmetry and position differences............ 86

4.3 Linear regression slopes ......................................... 86

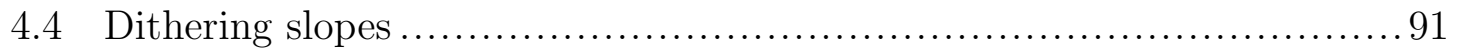

4.5 Summary of asymmetry calculations for the entire data set. The results are seperated by HRS, IHWP state and the type of correction applied (regression or dithering). The last column is the final correction applied, where the last entry is the final correction to the entire data set.

4.6 Summary of average values of $Q^{2}$ over several periods of time were the beam position was slightly different. Note that there is more data from the Right HRS because during that time, one of the sprecrometer magnets was malfunctioning.

4.7 Summary of HAMC simulation results for the acceptance factor $K$. The effects of small variations $( \pm 10 \mathrm{~mm}$ in both $\mathrm{x}$ and $\mathrm{y})$ in the dimensions of the collimator acceptance are also listed and are negligible...100 
4.8 All beam polarization measurements are summarized along with statistical errors of the partial Compton data. The errors quoted for the full data sets include both statistical and systematic contributions....

4.9 Summary of all the values used to calculate the final physics asymmetry, with their systematic errors...................................... 103

4.10 Summary of all contributions to the systematic error of $A_{P V}$

5.1 Values used for the proton and neutron electric and magnetic form factors.

5.2 Values used for kinematic constants determined during the experiment...106

A.1 Sample data set for PMT linearity tests at a photocathode input current of $10 \mathrm{nA}$. The double lines mark the acceptable linearity range.

A.2 Sample data set for PMT linearity tests at a photocathode input current of $5 \mathrm{nA}$. The double lines mark the acceptable linearity range.

A.3 Sample data set for PMT linearity tests at a photocathode input current of $1 \mathrm{nA}$. The double lines mark the acceptable linearity range.

B.1 Listed is a summary of the noise contribution analysis to the luminosity monitors for run 10270. At the time, the incoming beam current was $60 \mu \mathrm{A}$ incident on a thin lead target. Lumi1 was not functional. The top four lumis, which see the full luminosity of the beam, show that after taking into account all measurable sources of noise, there is an additional contribution of about $100 \pm 10 \mathrm{ppm}$ to all the signals. The bottom four lumis listed are those that are either blinded or have a $10 \%$ filter installed.

B.2 Summary of the noise contribution analysis to the luminosity monitors for run 10296. At the time, the incoming beam current was $60 \mu \mathrm{A}$ incident on a thin lead target and the bottom three lumis were shielded. The top four lumis, which see the full luminosity of the beam, show that after taking into account all measurable sources of noise, there is an additional contribution of about $100 \pm 10$ ppm to all the signals. ....134 
B.3 Asymmetry RMS values for luminosity monitor signals. Data was taken with a $60 \mu \mathrm{A}$ beam current and a thin lead target. The values of photocathode current were estimated by using gain curves for all the PMTs used.................................................... 135

D.1 Summary of fan speed boiling test. The data shows that the effects of the fan speed on the detector widths is barely noticeable. For this

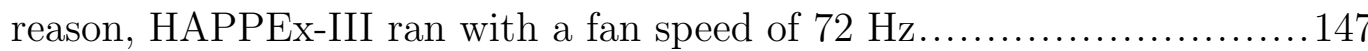




\section{List OF FiguRES}

Figure Page

1.1 On the left is Bohr's model and on the right is the 'cloud' model, with each energy level shown as three dimensional probability distributions.... 3

1.2 Examples of spherically symmetric charge distributions with their corresponding form factors in the Born approximation $[1] \ldots \ldots \ldots \ldots \ldots \ldots . \ldots$

1.3 This picture, taken from Hofstadter's Nobel lecture [2], shows a summary of charge density distributions found by studying form factor data obtained via electron scattering experiments...................... 6

1.4 Cross-section data for electron scattering from the proton. The three theoretical curves show expected values for (a) Mott curve for spin-less point proton, (b) point proton with Dirac magnetic moment and (c) point proton having anomalous Pauli contribution and Dirac magnetic moment. The experimental curve deviates from all three, leading to the need for a proton form factor to describe its own substructure.

1.5 Artist's rendition of the Quark model for a Carbon-12 nucleus [3]. For this isotope of Carbon, there are 6 protons and 6 neutrons, each composed of up (blue) and down quarks (red). The clouds around each quark represent the virtual gluons that keep them together.

1.6 Feynman diagram of the negative beta decay process. It was not until the development of the electroweak theory that the interaction was understood as an exchange of a $\mathrm{W}$ boson.................................. 11

1.7 Feynman diagrams for electromagnetic and weak electron-proton scattering.............................................................. 13

1.8 Results for the linear combination of strange form factors from several parity violation experiments conducted at $Q^{2} \approx 0.1 \mathrm{GeV}^{2}$. The ellipse is a $95 \%$ confidence region and points are located at the best fit value. ... 19

1.9 Results for HAPPEx, G0 and A4 for the linear combination of strange form factors. The dotted lines show a possible trend with increasing $Q^{2}$. The proposed HAPPEx III error is shown, placed at zero............20 
1.10 Predictions of the difference between proton radius and neutron radius for nuclei with mass number $A$. The black data points represent the relativistic mean field NL1 model and the gray points are for the nonrelativistic Skyrme sk-iii interaction. The data point at $R_{n}-R_{p}$ is included to show the impact of the proposed accuracy of the PREx measurement. The calculations used to make this figure can be found in $[12]$.

1.11 Flow chart of the physics data analysis of a neutron radius experiment. It shows how one can find a value for the RMS neutron of ${ }^{208} \mathrm{~Pb}$ by using a measurement of the neutron form factor.

1.12 Neutron form factor for ${ }^{208} \mathrm{~Pb}$ at the momentum transfer of the PREx experiment vs. the RMS neutron radius of ${ }^{208} \mathrm{~Pb}$ for a variety of models $[13]$

2.1 The layout of the entire HAPPEx experiment, emphasizing the components along the beamline and inside Hall A.

2.2 Schematic of source setup shows how the Hall A laser gets used to create a polarized beam of electrons [57]. A similar setup was also used for the previous HAPPEx experiments.

2.3 Diagram of the allowed energy transitions for Gallium Arsenide

2.4 Layout of CEBAF shows the race-track path taken by the electron beam, made up of two linacs and five recirculating arcs connecting them at each end.

2.5 Schematic of Hall A beamline shows location of important beam monitors and modulation coils.

2.6 The Stripline Beam Position Monitors are composed of four wires located symmetrically around the beamline, labeled as the Z-axis.

2.7 Sample plots of beam modulation response. Red and blue data represent horizontal and vertical modulation, accordingly. The magenta data is energy modulation. These plots are representative of one 'dithering' cycle.

2.8 Picture of the cryogenic target ladder inside the scattering chamber 40 
2.9 Detailed diagram of the magnetic components that make up each High Resolution Spectrometer [15].

2.10 Side and top view of the Vertical Drift Chambers used in each Spectrometer.

2.11 The HAPPEx detectors are composed of alternating layers of Lead and Acrylic and a 5-inch PMT housed inside an Aluminum frame.

2.12 Data taken using the standard Hall A detector package and the Counting DAQ. This data shows the alignment of the elastic peak in each Spectrometer.

2.13 Diagram of all HAPPEX DAQ components. 46

2.14 Displayed above are all four signals related to the pseudo-random helicity flips of the polarized electron beam. The top signal, labeled 'Helicity' defines the state of the beam polarization. The other signals are used to define the begining of a window, a pair and a quad (two pairs).

2.15 Circuit diagram of the integrating circuit for the 16-bit ADCs. 47

2.16 (a) Side view and (b) top view of the Moller Polarimeter. Each plot shows simulated events that hit the detectors. .50

2.17 The Compton Polarimeter is located where the beam enters Hall A. .....5 51

2.18 A detailed schematic of the Compton Polarimeter components............ 51

2.19 Schematic of beam pipe showing location of luminosity monitors relative to the target .................................................... 52

2.20 Simple schematic of a singleLuminosity Monitor

3.1 Diagram showing all the optical elements used as part of the Double Wien filter setup at the Injector. .56

3.2 Schematic of a cross-section of the PREx target ladder. .58

3.3 Picture of Copper frame used to house the PREx targets. Note the Liquid Helium intake at the top of the frame. .58 
3.4 Time-dependant target rate measured using counting mode DAQ for the three different Lead-Diamond targets. The target with the thinnest diamond backing ( $4.5 \%$ background) degraded the fastest. Two of the targets melted. The target with the thickest diamond (8\%) did not melt and ran for 4 days at $70 \mu \mathrm{A}$ (and 7.5 days total)

3.5 Variations in the RMS of the measured detector asymmetry throughout all the data runs. The sudden jump to RMS higher than $300 \mathrm{ppm}$ is a clear indicator of the degradation of the target. While most of the data was taken at $50 \mu \mathrm{A}$, runs higher than 4660 were conducted at 70 $\mu \mathrm{A}$.

3.6 Raster scan conducted with the Hall A standard DAQ package shows lower rates at the center of the rectangular raster pattern, indicating degradation of the target and the first steps of melting.

3.7 The left plot shows the correlation in the pulse-pair asymmetry between detectors in the left and right HRS after target degradation. The right plot shows the same signals after synching the raster with the helicity flip rate. These show that this technique effectively cancelled out the effects of target density fluctuations.

3.8 Graphical wire frame designs of the new Septum magnet.

3.9 Location of the focal plane detectors with respect to the vertical drift chambers and the $\mathrm{S} 0$ scintillator.

3.10 Final detector assembly in one of the spectrometers. The $A_{T}$ detector is set up on the yellow beam on the left. The $A_{T}$ and the focal plane detectors are placed on remotely controlled $\mathrm{x}$ - and $\mathrm{y}$ - tracks to facilitate proper alignment with the appropriate signals. ...

3.11 Signal profile for one of the 'thin' quartz detectors, referred to in this plot as lowerQuartz. By dividing the RMS by the mean of the histogram fit, one gets the energy resolution $\sigma_{E} / E$ of the detector.

3.12 Partial circuit diagram of one of the input channels on an 18-bit ADC. The resistor labeled R159 was changed to adjust the maximum current range.

3.13 Pedestal differences in ppm as the input current is increased. 
3.14 Plots (a), (b) and (c) show gaps in the data consistent with missing bits. These gaps go away when DAC is used (d) . .

3.15 When using a slowly varying DAC (left), the histogram of a sine wave is a smooth secant distribution (right).

3.16 The top plot shows the pedestal signal from one of the 18-bit ADC channels. The bottom plot shows the same channel after a pulser signal has been connected on an adjacent channel. No significant effect is observed.

3.17 Black box setup for PMT linearity studies.

3.18 Picture of the linearity test experimental setup. PMT shown was 5inch Burle used in HAPPEx-III.

3.19 Plot on the left shows how the signal varied when the filter returned to the same setting before installing a small aperture. On the right, with the aperture, the signal is now stable.

3.20 Sample raw data from linearity test. The signal is pseudo-randomly varied between six filter settings.

3.21 For the linearity tests, asymmetries are calculated for each filter attenuation and analyzed for any variations. ...

3.22 By fitting the measured asymmetries with respect to the average of $N^{+}$and $N^{-}$, one can extract a slope that represents the degree of nonlinearity for these running conditions.

4.1 This beam current monitor (BCM) data is from a run with a beam trip. The data in black is usable data, while the red data is what was cut out before the analysis was done. All data below 22000, as well as 10 windows before and 40 windows after was marked as 'bad' data.

4.2 Sign-corrected asymmetries, in units of parts-per-million, calculated for all data that passed the most basic data quality cuts. Modulation data is included. 
4.3 Summary plots for the main beam current and beam position monitors used in the final HAPPEx-III analysis. The data in blue was taken with IHWP OUT and the red data was taken with IHWP IN. Note that when averaging both sets of data, the position differences go to zero (black line).

4.4 Sign-corrected regressed asymmetries, in units of parts-per-million, calculated for all data that passed all data quality cuts.

4.5 Sample plots from one dithering cycle. The red data represents kicks in the $\mathrm{x}$-direction, blue represents the $\mathrm{y}$-direction and magenta regresents small energy shifts.

4.6 These plots show the effect of the modulation coils on the beam slope and position at the target.

4.7 Detector signal during a dithering cycle. Note that there is no obvious effect on the detected flux, allowing for this data to be used as food production data.

4.8 Sign-corrected, dither-adjusted asymmetries, in units of parts-per-million, calculated for all data that passed all data quality cuts.

4.9 Both plots show $Q^{2}$ histograms for the Left (red) and Right (blue) HRS. The plot on the right shows the histogram after weighing, which corrects for the attenuation along the detector.

4.10 Average weighted (black triangles) and unweighted (blue circles) $Q^{2}$ values for at data taken throughout the experiment. The variations seen can be explained by differences in the beam position.

4.11 Measurements of the probability for a particle to re-scatter into the detector with respect to the percentage deviation from the nominal beam momentum.

4.12 Energy Spectrum at HAPPEx III kinematics. (Red line - SLAC Model, Blue line - Hall C Model).

4.13 Both plots show simulation results for the vertex asymmetries for both HRS. They also show the results when varying the spectrometer acceptance by $\pm 10 \mathrm{~mm}$ in both the $\mathrm{x}$ and $\mathrm{y}$ directions. These values are conpared with the asymmetries of the observed $Q^{2}$ averages. .100 
4.14 Plot showing the entire collection of Moller and Compton beam polarization measurements throughout HAPPEx-III. The data is divided into four sections, each corresponding to a different beam tune.

5.1 Results from A4, G0 and HAPPEx-H for strange quark vector formfactors. The green and yellow shaded regions represent G0 correlated error and form factor errors.

5.2 Results from the SAMPLE hydrogen (blue lines) and deuteron (red lines) data. Also shown is the theoretical expected value for $G_{A}^{e(T=1)}$ (green lines)

5.3 One sigma results for the linear combination $G_{M}^{s}+\eta G_{E}^{s}$ for A4 backward (solid band) and forward angle (hatched band) measurements at $Q^{2} \approx 0.22 \mathrm{GeV}^{2}$. The ellipses represent the $68 \%$ and $95 \%$ confidence levels. Also show are several theoretical predictions.

5.4 The three plots above summarize the results of the G0 forward- and backward-angle measurements of the strange and anapole form factors (blue data point). The shaded bars represent global systematic uncertainties for those points. Included are results from A4 and SAMPLE, as well as low Q fit points from [48]. Some theoretical calculations are also included $[49,50]$

5.5 Results for all measurements of strange form factors from several parity violation experiments conducted at $Q^{2} \approx 0.1 \mathrm{GeV}^{2}$. The yellow and blue ellipses are $68 \%$ and $95 \%$ confidence level fits. Note that zero strangeness $\left(G_{E}^{s}=G_{M}^{s}=0\right)$ is at the edge of the $95 \%$ C. L. contour. .....114

5.6 The world data of the neutral weak effective couplings. The dotted contour shows a $95 \%$ confidence level fit of all the data before including results from parity violating electron scattering experiments. The filled contour represents the new constraint provided by PVES results, while the blue contour shows the 95\% CL fit of the entire data set.

5.7 Current experimental constraints on the strange form factors when considering all the existing data at $Q^{2} \approx 0.62 \mathrm{GeV}^{2}$. These include bands from the HAPPEx-III and G0 forward- and back-angle measurements. ...116 
5.8 Plotted is the entire set of world data for forward-angle measurements of linear combinations of strange vector form factors. This is an updated version of Figure 1.9 and includes the HAPPEx-III measurement (red data point), which further constrains the linear fit shown, moving it closer to zero.

5.9 Difference error when comparing the 'no strangeness' asymmetry to the measured physics asymmetry for all HAPPEx results. All these measurements are consistent with zero strangeness.

5.10 The red data point shows the final asymmetry result of the PREx experiment. The pink data point shows the proposed error for a PRExII result. Also shown are theoretical values from 8 selected models. The $R_{n}=R_{p}$ line represents the asymmetry if there was no neutron skin.

5.11 Layout of the MOLLER experiment, to be conducted in Hall A at Jefferson Labs. From left to right, it shows the target chamber, toroidal spectrometer and detector package. ...

A.1 Linearity test conducted at $\mathrm{HV}=700$ for an input current of $\approx 20 \mathrm{nA} \ldots 124$

A.2 Linearity test conducted at $\mathrm{HV}=700$ for an input current of $\approx 40 \mathrm{nA} . .124$

A.3 Linearity test conducted at $\mathrm{HV}=700$ for an input current of $\approx 60 \mathrm{nA} \ldots 125$

B.1 All eight lumi signals with respect to a beam current monitor signal. For the odd numbered lumis, which had no ND filters installed, the signals are extremely nonlinear and actually drop when the current is increased.

B.2 Both plots show data taken with one of the luminosity monitors during commissioning. When plotted against one of the BCM signals, certain targets give better linear responses than others

B.3 Luminosity Monitor configuration for the noise level tests. Originally, Lumi 4 had a 10\% filter. Eventually, it was blinded, and later lead bricks were place in front of Lumis 4,5 and 6 in an attempt to minimize background contributions. 
B.4 Summary of luminosity monitor data taken with a carbon target at several beam currents. The lumi signals (y-axis) go up with beam current (x-axis), as expected.

B.5 Summary of noise levels for all the luminosity monitor data taken with carbon. The fits show how the RMS values (y-axis) should decrease as beam current goes up (x-axis).

C.1 Scope trace of main Synchronization Monitor signals. Shown are the MPS signal (pink), GATE (yellow) and its compliment (blue)..............138

C.2 Zoomed in version of Figure C.1. Note that the beginning of the gate starts in the middle of the MPS 'settle time'............................ 138

C.3 Schematic of all the electronic elements used for the main Synchronization Monitor [57].

C.4 Correlations for all GATE and GATE signals from the Left and Right Spectrometers and the Counting House DAQ. The column on the left shows all the GATE correlations. The column on the right shows all the GATE correlations.

C.5 This set of plots shows another method of making sure all the electronics are synchronized, by studying how well correlated the plots in Figure C.4 really are. As expected, most of the data is at '1' and the rest converges as the frequency signal is increased.

C.6 Sample set of plots used to determine the synchronization between the Right Spectrometer and the Counting House crates.

C.7 Example of plots monitored by the shift crew to make sure synchronization between the electronics crates was good.

D.1 RMS width of asymmetries as measured by Detector 1, located in the L-HRS. The blue curve represents data taken at several different currents. The red curve is the expected statistical width according to Poisson statistics. The difference in these curves is caused by target 'boiling' fluctuations. 
D.2 RMS width of the sum of all eight lumi signals. Assuming dominance of statistics at $10 \mathrm{nA}$, data points at very low current behave properly (red curve), but at higher currents, target density fluctuations add non-statistical noise to the distribution. .............................. 146

D.3 RMS width of asymmetries as measured by both detectors with different raster sizes while running at $70 \mu \mathrm{A}$ with a fan speed of $72 \mathrm{~Hz}$. 148

D.4 RMS width of the sum of all eight lumi monitor signals with different raster sizes while running at $70 \mu \mathrm{A}$ with a fan speed of $72 \mathrm{~Hz} \ldots \ldots \ldots \ldots . . .148$ 


\section{CHAPTER 1}

\section{HISTORICAL BACKGROUND AND PHYSICS MOTIVATION}

\subsection{Introduction to Atomic Structure}

For the longest time, it was believed that the atom was the smallest component of matter. In fact, the word itself is derived from the Greek word atomos, meaning 'undivisible.' The concept of the atom was proposed by the Greek philosophers Democritus and Leucippus over two thousand years ago, but it was not until the early 19th century that discoveries in the field of chemistry provided proof of such a fundamental particle.

English chemist John Dalton found that chemical elements were composed of unique types of atoms, and that these elements could be combined in specific ratios to form more complex chemical compounds. The subsequent discoveries of the electron by J.J. Thomson (1897) and the nucleus by Ernest Rutherford (1909) provided evidence of the existence of the atom's substructure. These were the first steps toward developing a complete theory of atomic structure.

J.J. Thomson was the first to propose that an atom was made up of smaller, more fundamental particles that were over a thousand times smaller than Hydrogen, the smallest atom. He conducted an experiment that not only proved this, but also provided the charge-to-mass ratio of these newly discovered particles. Even though he determined that atoms were made up of these tiny negatively charged particles, he still had to explain how atoms could have no net charge. He proposed that the electrons were distributed among a sea of uniform positive charge, like plums in a plum pudding.

Not long after Thomson proposed his model for the atom, Ernest Rutherford, one 
of his former students, conducted an experiment that would provide evidence that the positive charge was not uniformly distributed inside the atomic volume. The experiment consisted of shooting a beam of positively charged alpha particles at a gold foil and studying their scattering angle, if any, after interacting with the gold atoms. If Thomson's model was right, the alpha particles would barely be deflected by the electrostatic forces of the gold atom's positive charge.

Rutherford's experiment showed that this was mostly true, but that there were a few alpha particles that were scattered at very large angles. He explained this by proposing that the positive charge had to be concentrated in a very small radius at the center of the atom. Using the equations of motion to describe the interaction between two point-like particles, he developed an equation to model the scattering, now famously known as the Rutherford Cross-Section

$$
\frac{d \sigma}{d \Omega}=\left(\frac{Z_{1} Z_{2} \alpha}{2 m v^{2} \sin ^{2} \theta / 2}\right)^{2}
$$

where $Z_{1,2}$ are the atomic numbers of both elements involved in the scattering process, $\theta$ is the scattering angle in the lab frame, $\alpha$ is the fine structure constant, $m$ is the mass of the alpha particle and $v$ is its velocity.

Data collected by Rutherford's colleagues, Geiger and Marsden, showed the validity of this equation. With it, they established an upper limit for the radius of the nuclear core of about $10^{-14} \mathrm{~m}$, ten thousand times smaller than the atom itself. Scattering experiments such as this one became the best tool for probing such small distance scales.

\subsection{Modeling the Atom and its Components}

By 1912, physicist Niels Bohr had developed a theory that explained how the negatively charged electrons interacted with the positively charged nucleus. The theory described how electrons maintain stable orbits around the nucleus (see Figure 1.1), characterized by unique sets of quantum numbers. Bohr also proposed that an electron could move to a lower energy orbit by emitting a photon, giving rise to the spectral lines of the hydrogen atom. Other contributions by Schrodinger and Heisenberg in the mid-1920s painted a much more complex picture of the atom, describing it as a 'cloud' of electrons surrounding the nucleus. This cloud is a probability dis- 


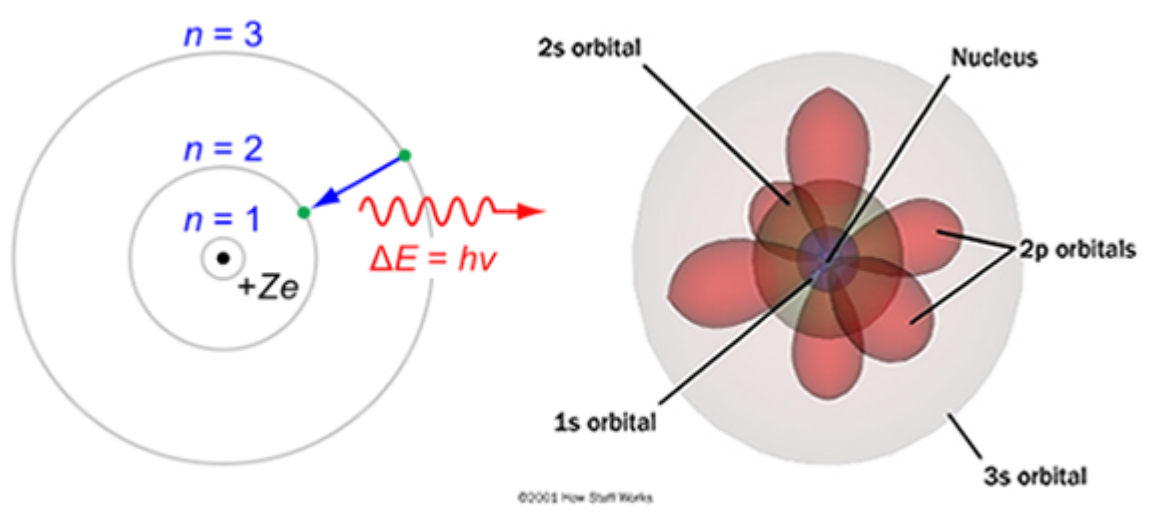

Figure 1.1: On the left is Bohr's model and on the right is the 'cloud' model, with each energy level shown as three dimensional probability distributions.

tribution that defines the most likely locations of the electrons.

\subsubsection{The Electron}

While Schrodinger's equation helped model electron behavior using wave mechanics, Heisenberg developed a theory using matrix mechanics which described electrons as particles, leading to the concept of particle-wave duality. Both of these turned out to be successful in describing mathematically the interactions between electrons in simple atoms.

The most accurate mathematical interpretation of the electron was developed in 1928 by Paul Dirac, who was successful in creating an equation that was consistent with the principles of quantum mechanics and the theory of special relativity. This great achievement led the way toward the development of Quantum Electrodynamics (QED) in the 1940s, a theory that fully accounts for all interactions between matter and light via the exchange of virtual photons.

QED allowed for physicists to make accurate calculations and predictions about scattering experiments. Eventually, the alpha particle in scattering experiments would be replaced with electrons that could be delivered as high energy beams. Due to the electron's particle-wave nature, by which as a particle's energy is increased, its DeBroglie wavelength decreases, a beam of electrons can interact at very small scales and resolve the inner structure of the nucleus. 
For high energy electron scattering, the Rutherford cross-section must be modified to account for relativistic spin effects of the electron. The new expression is called the Mott cross-section

$$
\left(\frac{d \sigma}{d \Omega}\right)_{M o t t}=\frac{4 Z^{2} \alpha^{2} E^{2}}{q^{4}} \cos ^{2} \frac{\theta}{2},
$$

where $Z$ is the atomic number of the target nucleus, $\alpha$ is the fine structure constant, $E$ is the energy of the electron, $\theta$ is the scattering angle and $q$ is a new concept called the 4-momentum transfer. An electron interacts with a nucleus by exchanging a virtual photon with a point-like nucleus in the target medium. The 4-momentum transfer is the magnitude of 4-momentum the scattered electron loses via this electromagnetic interaction and is defined as

$$
q^{2}=-4 E E^{\prime} \sin ^{2} \frac{\theta}{2}
$$

where $E$ is the energy of the incoming electron, $E^{\prime}$ is the energy of the scattered electron and $\theta$ is the angle by which the outgoing electron was deflected. Electron scattering becomes a more useful tool for studying nucleon structure as the momentum transfer is increased, providing a greater resolution at smaller distance scales.

\subsubsection{The Nucleus}

After Rutherford's discovery that the positive charge of an atom was located in a small and massive central core, questions arose as to what it might be composed of. To delve into the structure of the nucleus itself, alpha scattering experiments were continued with various targets. By 1932, Rutherford and another colleague James Chadwick had a clear picture of the nucleus, composed of positively charged protons and neutrons with no charge, both particles having very similar masses.

Over the next few decades, the structure of the nucleus was confirmed by several high energy electron scattering experiments. The first high energy cross-section measurements were conducted in the 1950s at a linear accelerator at Stanford University. Such data showed a divergence from the Mott cross-section formula, indicating that the nucleus was not a point-like structure like the electrons.

At sufficiently high energies, electrons interact with the charge components of the nucleus, instead of the nucleus as a whole, allowing for examination of its substructure. To account for these interactions, the Mott cross section formula must be modified 


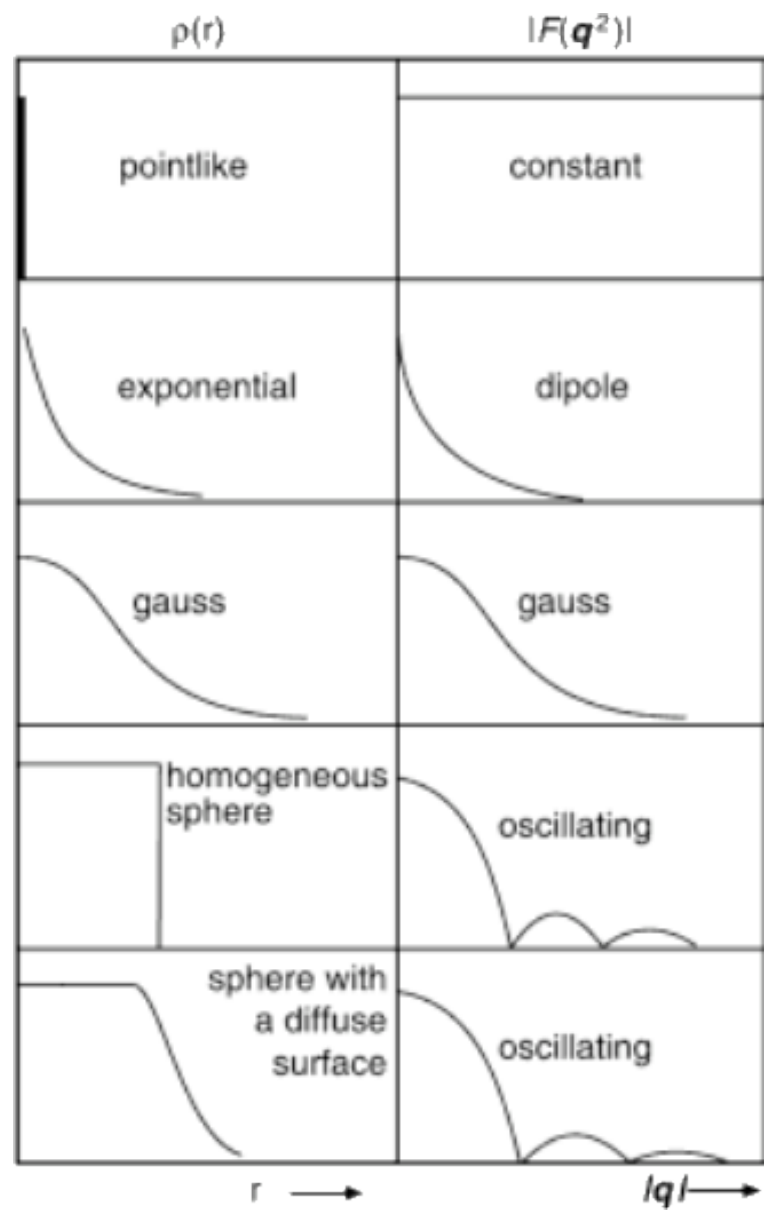

Figure 1.2: Examples of spherically symmetric charge distributions with their corresponding form factors in the Born approximation [1].

by using a function called a form factor

$$
\frac{d \sigma}{d \Omega}=\left(\frac{d \sigma}{d \Omega}\right)_{M o t t}|F(q)|^{2} .
$$

In the Born approximation, which describes the electron wave-functions as plane waves and assumes negligible nuclear recoil, the form factor is defined as the Fourier transform of the electric charge distribution of a nucleus. These functions depend on the resolution of the scattering experiment, which increases as the 4-momentum transfer of the interaction is increased. Figure 1.2 shows how form factor functions vary for several possible charge distributions.

Data taken at a wide range of $q^{2}$ can be used to show that the charge of the nucleus is distributed as a homogeneous sphere with a diffuse surface. Large amounts 


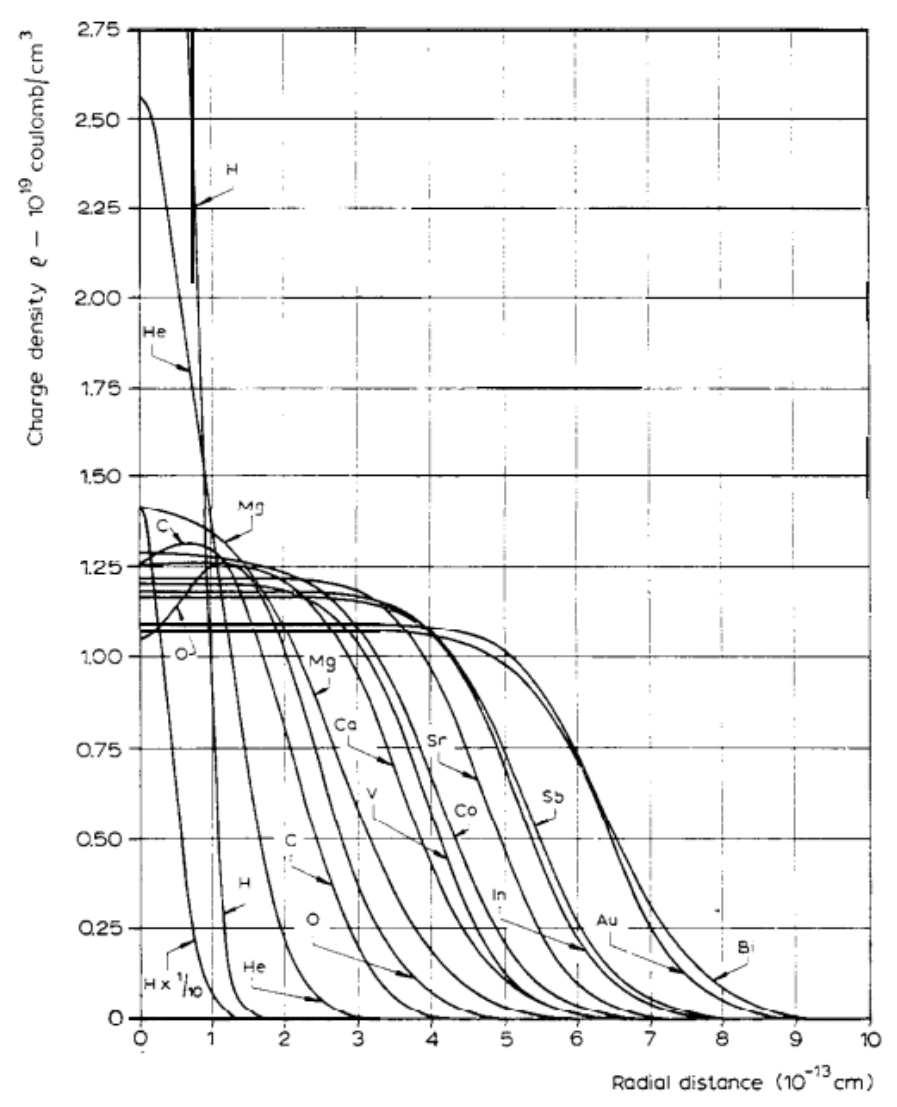

Figure 1.3: This picture, taken from Hofstadter's Nobel lecture [2], shows a summary of charge density distributions found by studying form factor data obtained via electron scattering experiments.

of cross-section data have helped physicists paint a clear picture about the structure of a wide range of atomic nuclei. In 1961, Robert Hofstadter was awarded the Nobel Prize for his work interpreting electron scattering data and providing a clear picture of nuclear structure. Not only did he derive charge distributions for numerous atomic nuclei (see Figure 1.3), he also showed that protons had finite size and therefore were composed of even smaller particles (see Figure 1.4).

During the time in which so many experimental results led to the understanding that an atom is the result of electromagnetic interactions between electrons and the protons and neutrons in the nucleus, others were attempting to explain how protons can remain so tightly packed inside the nucleus. A new strong force was therefore proposed to explain why protons do not repel each other at nuclear distance scales. Hideki Yukawa was one of the first to make an attempt at creating a model to explain 
how this new force kept nucleons together.

\subsection{Understanding Nucleon Structure}

In 1934 Yukawa proposed a new particle called a meson which acted as the carrier of the strong force in the same way that the photon is the carrier of the electromagnetic force. Nucleons were thought to be kept together by a constant exchange of these mesons. One of the first steps at obtaining proof of this new force was the discovery in 1947 of the pion, a particle with the predicted properties of Yukawa's meson. Once particle accelerator experiments could be conducted at high enough energies, physicists were able to gain a deeper understanding of how the strong force actually worked.

Results from various deep inelastic scattering (DIS) experiments finally provided direct evidence of point-like particles inside the protons and neutrons. Such experimental results, along with contributions by James Bjorken and Richard Feynman led to the development of Quantum Chromodynamics (QCD) in the 1970s, a theory that defines the strong force as the interactions between quarks and gluons inside hadrons. According to QCD, protons and neutrons are hadrons composed of three valence quarks and gluons, which are the true mediators of the strong force. It was later understood that the force that keeps nucleons together is a residual force caused by strong interactions between their constituents.

It is currently understood that all matter is made up of quarks and leptons, kept together by strong and electromagnetic interactions. Figure 1.5 shows what a Carbon nucleus might look like when considering its quark structure. While these theories have been very successful in explaining the structure of atoms, questions still remain about how the properties of individual quarks contribute to the observed properties of nucleons. For example, we know through several experiments that certain nucleon properties, such as mass and spin, arise not only from valence quarks (two up and one down for the proton, two down and one up for the proton), but from interactions inside a 'sea' of gluons and other quark-antiquark pairs. 


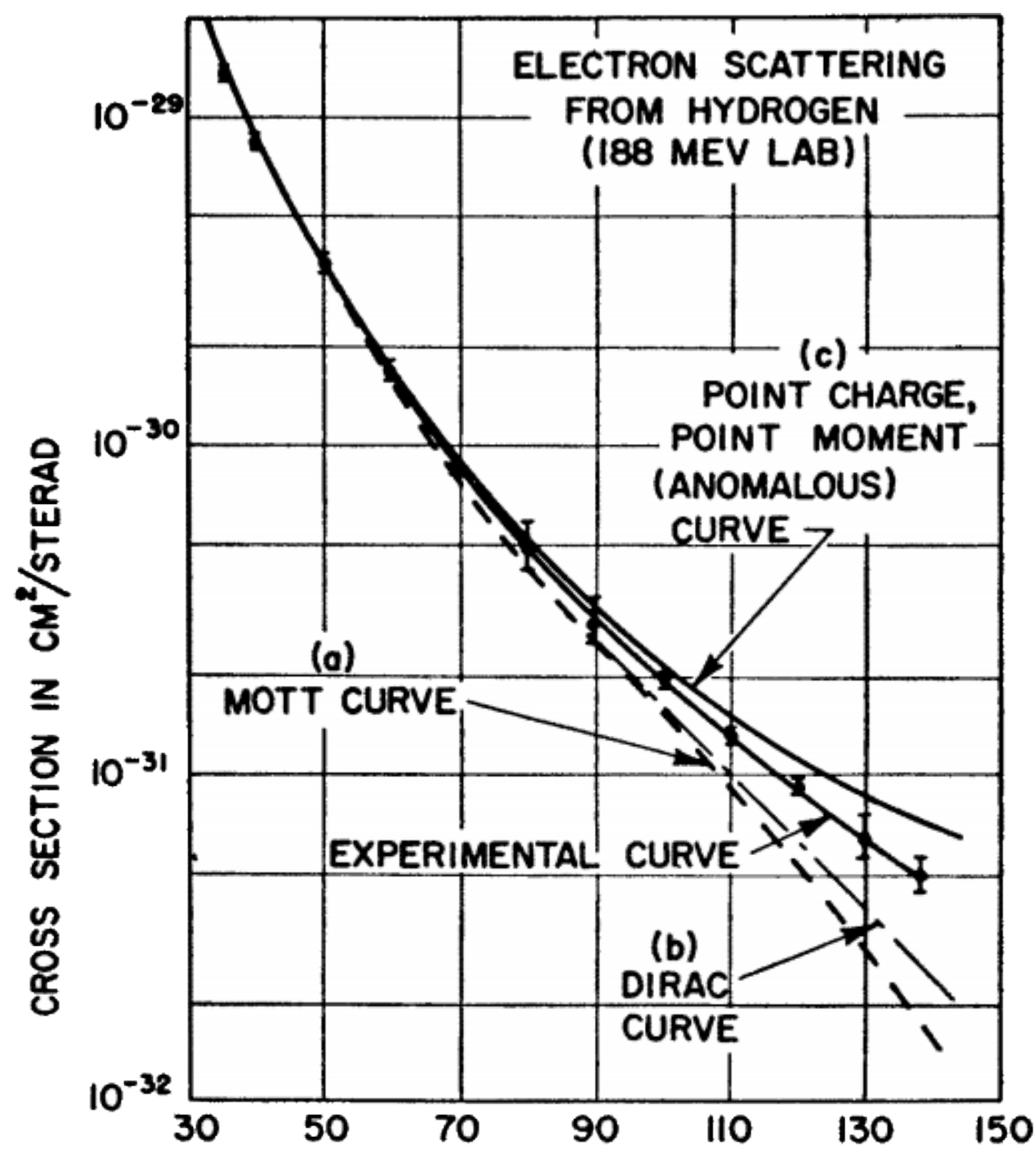

\section{LABORATORY ANGLE OF SCATTERING (IN DEGREES)}

Figure 1.4: Cross-section data for electron scattering from the proton. The three theoretical curves show expected values for (a) Mott curve for spin-less point proton, (b) point proton with Dirac magnetic moment and (c) point proton having anomalous Pauli contribution and Dirac magnetic moment. The experimental curve deviates from all three, leading to the need for a proton form factor to describe its own substructure. 


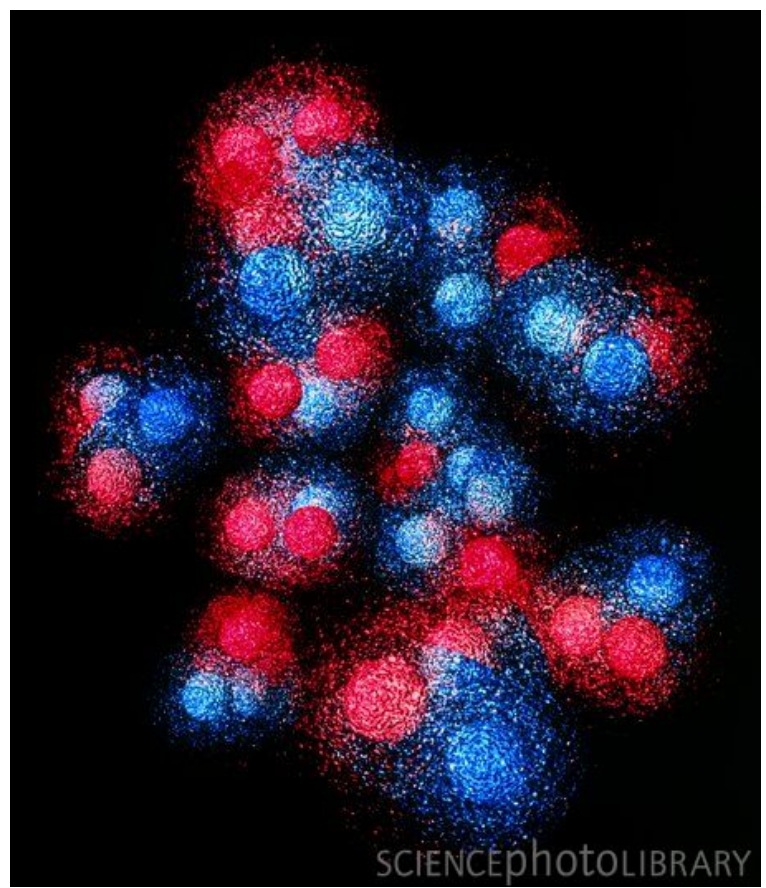

Figure 1.5: Artist's rendition of the Quark model for a Carbon-12 nucleus [3]. For this isotope of Carbon, there are 6 protons and 6 neutrons, each composed of up (blue) and down quarks (red). The clouds around each quark represent the virtual gluons that keep them together. 


\subsection{The Weak Interaction and Parity Violation}

The weak force was developed to explain nuclear beta decay, the process that governs the radioactive decay of an element with $\mathrm{Z}$ protons

$$
{ }^{A} Z \rightarrow{ }^{A}(Z+1)+\beta^{-}+\overline{\nu_{e}} .
$$

The weak force was originally described in 1933 by Enrico Fermi as a four-fermion contact interaction, a process by which a neutron turns into a proton by emitting an electron and an antineutrino. The existence of the neutrino, a neutral particle with practically no mass, had been proposed by Pauli in order to preserve the principle of energy and momentum conservation in the beta decay process.

It was later shown by Glashow, Weinberg and Salam that at very short distance scales, comparable to the size of the nucleus, the weak and electromagnetic forces are different manifestations of the same force. This unified model referred to as the electroweak theory, predicted the existence of several heavy intermediate bosons that work as mediators for the weak force. There are three types of heavy bosons: a positive and negative $\mathrm{W}$ boson, which take part in charged weak interactions such as beta decay (see Figure 1.6), and the $\mathrm{Z}$ boson which mediates neutral weak interactions like electron-electron scattering.

While physicists had been aware of the interactions that were mediated by the $\mathrm{W}$ boson, the existence of a weak neutral current was an important new idea that was produced to make the electroweak unification possible. A weak neutral current interaction was first observed at CERN in 1973, proving its existence and showing the robustness of the theory. The $\mathrm{W}$ and $\mathrm{Z}$ bosons were not to be observed until the mid-1980s when there was finally a particle accelerator of sufficient energy to produce them.

A peculiar feature of the weak force is that it violates one of the basic principles of conservation laws in physics, all of which are related to symmetries of nature. Conservation laws imply that certain mathematical values are preserved when a system is transformed in some way [4]. For example, translations in a homogeneous space give rise to the conservation of linear momentum. There are also discrete symmetries that describe invariances in non-continuous systems. A change such as $\vec{x} \rightarrow \overrightarrow{-x}$ gives rise to parity conservation, the idea that the laws of physics are the same under spatial inversions. Until the mid-1950s, it was believed that all forces conserved parity. 


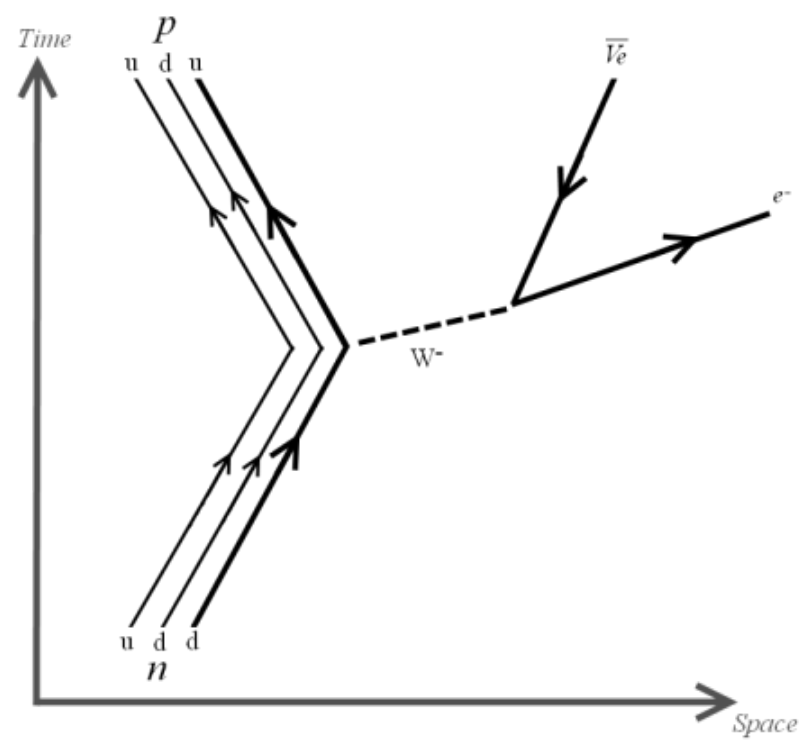

Figure 1.6: Feynman diagram of the negative beta decay process. It was not until the development of the electroweak theory that the interaction was understood as an exchange of a $\mathrm{W}$ boson. 
In 1956, Lee and Yang published a paper questioning parity conservation in weak interactions and suggested several experiments to put this matter to rest [5]. One of these experiments involved studying the beta decay of Cobalt-60. The idea was to use a magnetic field to align the spin of the nuclei in the same direction. If parity is conserved, then the beta rays should be emitted at the same rate from both poles of the nuclei. Any deviation would be evidence that parity is indeed violated in the weak interaction.

This experiment was carried out by Madame Wu of Columbia University and the results provided proof that parity was not conserved in the beta decay of Cobalt60. While the implications of this discovery were not immediately obvious, the parity violating nature of the weak interaction has become a very important tool for studying the structure of nucleons, as will be discussed in the next section.

The unification of the electromagnetic and weak forces, as well as the development of QCD have allowed nuclear physicists to develop a theory that explains all interactions of matter. The Standard Model has been very successful in describing all matter as being composed of quarks and leptons which interact by way of the electromagnetic, weak and strong forces through the exchange of bosons. Many predictions of the theory have been tested successfully through experimental means. Such a prediction, the existence of a Higgs boson responsible for giving particles their mass, is the focus of numerous contemporary collider experiments.

\subsection{Strangeness in Nucleons}

The substructure of the proton can be described as three 'valence' quarks, two up and one down, and a 'sea' composed of gluons and quark-antiquark pairs. It has been shown experimentally that all of the nucleon's properties cannot be fully explained without considering contributions from the quark and gluon sea. It was shown by the EMC collaboration that the valence quarks are not the dominant contribution to the nucleon spin [6], leading to experiments that try to characterize the role of strange quarks and gluons.

Kaplan and Manohar [7] suggested that one could use elastic neutral-current scattering experiments to extract information about the strange quark contributions to the proton. Measurements of the strange matrix element $\langle p|\bar{s} s| p\rangle$ through the pion- 


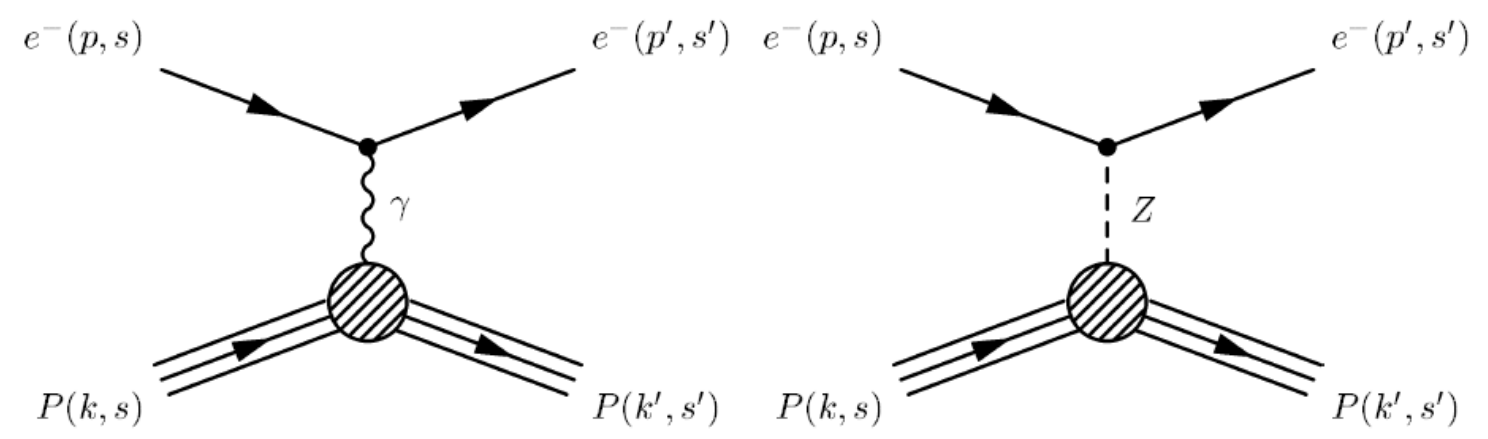

Figure 1.7: Feynman diagrams for electromagnetic and weak electron-proton scattering.

nucleon sigma term, and of $\left\langle p\left|\bar{s} \gamma_{\mu} \gamma_{5} s\right| p\right\rangle$, measured through elastic $\nu$ p and inelastic ep scattering, indicate nonzero values. These results show that there is a small but important strange quark contribution to the properties of the proton.

A parity violation experiment utilizing elastic polarized electron-proton scattering would lead to a third strange matrix element, $\left\langle p\left|\bar{s} \gamma_{\mu} s\right| p\right\rangle$, and, in combination with other non-PV experimental data, would provide information about the strange quark contribution to the electromagnetic nucleon form factors. A clear understanding of such contributions would help clarify results from other experiments, as well as improve our knowledge of sea quark contributions [?].

\subsection{Using Parity Violation to Probe the Nucleon}

When an electron is scattered by a proton, the interaction is mediated by either a photon or a $Z_{0}$ boson. Figure 1.7 shows the first level Feynman diagrams for these interactions. Interference between the electromagnetic and weak amplitudes give rise to a parity violating asymmetry that can be measured experimentally. The crosssection for elastic electron-proton scattering is proportional to the square of the total amplitudes

$$
\sigma^{R, L} \propto\left(\mathcal{M}_{\gamma}+\mathcal{M}^{R, L}\right)^{2}
$$

and can be used to define the parity violating asymmetry as

$$
A_{L R}^{e p}=\frac{\sigma^{R}-\sigma^{L}}{\sigma^{R}+\sigma^{L}} \propto \frac{\left(\mathcal{M}_{\gamma}+\mathcal{M}_{Z}^{R}\right)^{2}-\left(\mathcal{M}_{\gamma}+\mathcal{M}_{Z}^{L}\right)^{2}}{\left(\mathcal{M}_{\gamma}+\mathcal{M}_{Z}^{R}\right)^{2}+\left(\mathcal{M}_{\gamma}+\mathcal{M}_{Z}^{L}\right)^{2}} \approx \frac{\mathcal{M}_{Z}^{R}-\mathcal{M}_{Z}^{L}}{\mathcal{M}_{\gamma}}
$$


assuming that $\mathcal{M}_{Z} \ll \mathcal{M}_{\gamma}$. The size of the asymmetry can further be approximated to give us an order-of-magnitude estimate

$$
A_{L R}^{e p}=\frac{\mathcal{M}_{Z}}{\mathcal{M}_{\gamma}} \approx \frac{q^{2}}{M_{Z}^{2}} \approx 10 p p m,
$$

where $M_{Z}$ is the mass of the $Z_{0}$ boson and $q^{2} \approx 0.1 \mathrm{GeV}^{2}$ is the magnitude of the four-momentum transfer squared from HAPPEx-II.

In order to find the relationship between the measured asymmetry and the strange quark form factors, the electromagnetic and weak interactions must be examined and their amplitudes calculated.

\subsubsection{Electromagnetic Amplitude for Electron-Proton Scattering}

For the exchange of the photon, the electromagnetic scattering amplitude is

$$
\mathcal{M}_{\gamma}=j_{\mu}\left(\frac{1}{q^{2}}\right) J^{\mu}
$$

where $j_{\mu}$ is the electron current

$$
j_{\mu}=-e \bar{u}_{e} \gamma_{\mu} u_{e}
$$

$J^{\mu}$ is the proton current

$$
J^{\mu}=e \bar{u}_{p}\left(F_{1}\left(q^{2}\right) \gamma^{\mu}+\frac{i}{2 M_{p}} F_{2}\left(q^{2}\right) \sigma^{\mu \nu} q_{\nu}\right) u_{p}
$$

In Equation 1.10, $e$ is the electron charge and $u_{e}\left(\bar{u}_{e}\right)$ is the incoming (outgoing) electron's Dirac spinor. Equation 1.11 includes the Dirac and Pauli form factors $F_{1}$ and $F_{2}$, the proton mass $M_{p}$ and the proton spinor $u_{p}$.

The form factors are experimentally measured values dependent on $q^{2}$ used to account for the structure of the proton, since it is not a point-like particle like the electron. A commonly used linear combination of these form factors is known as the Sachs form factors,

$$
G_{E}^{p, n} \equiv F_{1}^{p, n}-\tau F_{2}^{p, n} \quad G_{M}^{p, n} \equiv F_{1}^{p, n}+F_{2}^{p, n}
$$

where $\tau=Q^{2} / 4 M_{p}^{2}, M_{p}$ is the mass of the proton and $Q^{2}=-q^{2}$. These are also known as the electric and magnetic form factors of the proton and neutron. When the 
momentum transfer is sufficiently low, the Sachs form factors can be interpreted as the Fourier transforms of the charge and magnetic distributions. In the limit where $q^{2} \rightarrow 0$, they take on the values

$$
\begin{aligned}
& G_{E}^{p \gamma}=1, \quad G_{M}^{p \gamma}=\mu_{p} \approx 2.79, \\
& G_{E}^{n \gamma}=0, \quad G_{M}^{n \gamma}=\mu_{n} \approx-1.91,
\end{aligned}
$$

where $\mu_{N}=e / 2 M_{p}$ is the nucleon magnetic moment and the superscript $\gamma$ indicates that the form factors refer to the electromagnetic reaction. By combining the Sachs form factors and Equation 1.9, a differential cross-section can be found for unpolarized electron-proton scattering, also known as the Rosenbluth formula [8]

$$
\overline{d \sigma}_{d \Omega b}=\left(\frac{\alpha^{2}}{4 E^{2} \sin ^{4} \theta / 2}\right) \frac{E^{\prime}}{E}\left\{\frac{\left(G_{E}^{p \gamma}\right)^{2}+\tau\left(G_{M}^{p \gamma}\right)^{2}}{1+\tau} \cos ^{2} \frac{\theta}{2}+2 \tau\left(G_{M}^{p \gamma}\right)^{2} \sin ^{2} \frac{\theta}{2}\right\}
$$

where $\alpha$ is the fine structure constant, $E$ is the energy of the incident electron, $E^{\prime}$ is the energy of the scattered electron, and $\theta$ is the scattering angle in the lab frame. Changing the superscript from $p$ to $n$ gives the appropriate cross section for neutrons.

More can be understood about the proton's substructure by expressing the proton current in term of the quark flavors that compose it.

$$
\begin{aligned}
J^{\mu} & =\left\langle p\left|\Sigma_{i=u, d, s} Q_{i} \bar{u}_{i} \gamma^{\mu} u_{i}\right| p\right\rangle \\
& =\bar{u}_{p}\left[\Sigma_{i=u, d, s} Q_{i}\left(F_{1}^{i} \gamma^{\mu}+\frac{i}{2 M_{p}} F_{2}^{i} \sigma^{\mu \nu} q_{\nu}\right)\right] u_{p}
\end{aligned}
$$

where $Q_{i}$ is the electric charge of quark $i, u_{i}$ is the quark spinor and $F_{1}^{i}$ and $F_{2}^{i}$ are the quark flavor form factors. Since the other quark flavors have mass $m_{q} \gg \Lambda_{Q C D}$, their contribution to the proton structure is negligible. $\Lambda_{Q C D}$ is a parameter known as the QCD scale and can be thought of as the boundary at which the strong coupling becomes small and quarks barely interact with each other.

The Sachs form factors can once again be used for convenience, this time expressing them in terms of the quark form factors

$$
G_{E, M}^{\gamma p}=\frac{2}{3} G_{E, M}^{u}-\frac{1}{3} G_{E, M}^{d}-\frac{1}{3} G_{E, M}^{s} .
$$

Because of charge symmetry between the quarks in the proton and neutron

$$
p \rightarrow n \Rightarrow u \rightarrow d, d \rightarrow u, s \rightarrow s
$$




\begin{tabular}{|c|c|c|}
\hline Fermion & $g_{V}$ & $g_{A}$ \\
\hline$\nu_{e}, \nu_{\mu}, \nu_{\tau}$ & +1 & -1 \\
\hline$e, \mu, \tau$ & $-1+4 \sin ^{2} \theta_{W}$ & +1 \\
\hline$u, c, t$ & $1-\frac{8}{3} \sin ^{2} \theta_{W}$ & -1 \\
\hline$d, s, b$ & $-1+\frac{4}{3} \sin ^{2} \theta_{W}$ & +1 \\
\hline
\end{tabular}

Table 1.1: Weak charges of leptons and quarks.

another equation can be found that relates the proton form factors to the quark form factors

$$
G_{E, M}^{\gamma n}=\frac{2}{3} G_{E, M}^{d}-\frac{1}{3} G_{E, M}^{u}-\frac{1}{3} G_{E, M}^{s} .
$$

Now that we have obtained two sets of linearly independent equations for the six quark form factors by studying the electromagnetic interaction, the weak interaction can be used to find a third set. Once this is done, the individual quark form factors can be used to understand each quark's contribution in terms of the Sachs form factors.

\subsubsection{Weak Neutral Interaction Amplitude}

When electron-proton scattering is mediated by the $Z^{0}$ boson, the amplitude of the interaction can be written for low $Q^{2}$ as

$$
\mathcal{M}_{Z}=j_{\mu}^{Z}\left(\frac{1}{M_{Z}^{2}}\right) J^{Z, \mu},
$$

where $j_{\mu}^{Z}$ is the weak electron current

$$
j_{\mu}^{Z}=\bar{u}_{e} \gamma_{\mu}\left(g_{V}^{e}-g_{A}^{e} \gamma_{5}\right) u_{e}
$$

The vector and axial weak charges, $g_{V}^{e}$ and $g_{A}^{e}$, for all the point-like fermions are summarized in Table 1.1. The electroweak mixing angle, $\theta_{W}$, is a parameter that describes how the weak and electromagnetic force couplings are related to each other. The proton current can be written as

$$
J^{Z, \mu}=\bar{u}_{p}\left[\gamma^{\mu} F_{1}^{Z}\left(q^{2}\right)+\frac{i}{2 M_{p}} F_{2}^{Z}\left(q^{2}\right) \sigma^{\mu \nu} q_{\nu}+\gamma^{\mu} \gamma^{5} G_{A}^{Z}+\gamma^{5} q^{\mu} F_{P}^{Z}\right] u_{p},
$$

where $F_{1}^{Z}, F_{2}^{Z}, G_{A}^{Z}$ and $F_{P}^{Z}$ are four proton weak form factors that depend only on $Q^{2}$. $G_{A}^{Z}$ is called the axial form factor, $F_{P}^{Z}$ is the induced pseudoscalar form factor and the 
weak neutral current form factors, $F_{1}^{Z}$ and $F_{2}^{Z}$, are analogous to the electromagnetic form factors that make up the Sachs form factors. Both the axial and pseudoscalar form factors end up contributing little to the proposed kinematics of the HAPPEx-III experiment.

The proton current can also be written in terms of the quark flavors, as was done in Equation 1.16, by using the weak charge $g_{V}^{i}$ instead of the electromagnetic charge $Q^{i}$

$$
J^{\mu}=\bar{u}_{p}\left[\Sigma_{i=u, d, s} g_{V}^{i}\left(F_{1}^{Z, i} \gamma^{\mu}+\frac{i}{2 M_{p}} F_{2}^{Z, i} \sigma^{\mu \nu} q_{\nu}\right)\right] u_{p} .
$$

Once again using the Sachs form factors, we finally obtain the last set of equations needed to determine each quark's electric and magnetic form factor in terms of the proton and neutron form factors

$$
G_{E, M}^{p Z}=\left(\frac{1}{4}-\frac{2}{3} \sin ^{2} \theta_{W}\right) G_{E, M}^{u}-\left(\frac{1}{4}-\frac{1}{3} \sin ^{2} \theta_{W}\right)\left(G_{E, M}^{d}+G_{E, M}^{s}\right) .
$$

Combining this expression with Equations 1.17 and 1.19 yields three sets of equations for the individual quark flavors

$$
\begin{aligned}
G_{E, M}^{u} & =\left(3-4 \sin ^{2} \theta_{W}\right) G_{E, M}^{p \gamma}-G_{E, M}^{p, Z} \\
G_{E, M}^{d} & =\left(2-4 \sin ^{2} \theta_{W}\right) G_{E, M}^{p \gamma}+G_{E, M}^{n \gamma}-G_{E, M}^{p, Z} \\
G_{E, M}^{s} & =\left(1-4 \sin ^{2} \theta_{W}\right) G_{E, M}^{p \gamma}-G_{E, M}^{n \gamma}-G_{E, M}^{p, Z} .
\end{aligned}
$$

Since we are interested in finding how the strange sea quarks contribute to the electric and magnetic properties of the proton, we can use the last expression, along with the previous derivations of the electromagnetic and weak form factors to find the dependance of the parity violating asymmetry to the strange form factors. By starting with the exact expression for the asymmetry [14], found to be

$$
A^{e p}=\frac{-G_{F} Q^{2}}{4 \pi \alpha \sqrt{2}}\left[\frac{\epsilon G_{E}^{\gamma p} G_{E}^{Z p}+\tau G_{M}^{\gamma p} G_{M}^{Z p}-\left(1-4 \sin ^{2} \theta_{W}\right) \epsilon^{\prime} G_{M}^{\gamma p} G_{A}^{Z p}}{\left.\epsilon\left(G_{E}^{p \gamma}\right)^{2}+\tau\left(G_{M}^{p \gamma}\right)^{2}\right)}\right],
$$

and rearranging Equation 1.27 into

$$
G_{E, M}^{Z p}=\left(1-4 \sin ^{4} \theta_{W}\right) G_{E, M}^{\gamma p}-G_{E, M}^{\gamma n}-G_{E, M}^{s}
$$

we arrive at

$$
\begin{aligned}
A_{L R}^{e p}= & {\left[\frac{-G_{F} Q^{2}}{4 \pi \alpha \sqrt{2}}\right]\left\{\left(1-4 \sin ^{2} \theta_{W}\right)-\frac{\epsilon G_{E}^{p \gamma}\left(G_{E}^{n \gamma}+G_{E}^{s}\right)+\tau G_{M}^{p \gamma}\left(G_{M}^{n \gamma}+G_{M}^{s}\right)}{\epsilon\left(G_{E}^{p \gamma}\right)^{2}+\tau\left(G_{M}^{p \gamma}\right)^{2}}\right.} \\
& \left.-\frac{\left(1-4 \sin ^{2} \theta_{W}\right) \epsilon^{\prime} G_{M}^{p \gamma}\left(-G_{A}^{(1)}+\frac{1}{2} G_{A}^{s}\right)}{\left.\epsilon\left(G_{E}^{p \gamma}\right)^{2}+\tau\left(G_{M}^{p \gamma}\right)^{2}\right)}\right\}
\end{aligned}
$$




\begin{tabular}{lr}
\hline Measured Asymmetry & $-22.1 \mathrm{ppm}$ \\
\hline Beam Energy & $3.4 \mathrm{GeV}$ \\
\hline Beam Current & $100 \mu \mathrm{A}$ \\
\hline Beam Polarization & $80 \%$ \\
\hline Target & $25 \mathrm{~cm} \mathrm{LH} 2$ \\
\hline Scattering Angle & $13.7^{\circ}$ \\
\hline Required Statistical Accuracy & $2.5 \%$ \\
\hline Detected Rate (each Spectrometer) & $2.2 \mathrm{MHz}$ \\
\hline Running Time & 30 days \\
\hline
\end{tabular}

Table 1.2: Summary of proposed kinematic conditions for HAPPEx-III.

where $\epsilon=\left[1+2(1+\tau) \tan ^{2}(\theta / 2)\right]^{-1}$ is the transverse polarization of the virtual photon exchanged and $\epsilon^{\prime}=\sqrt{\tau(1+\tau)\left(1-\epsilon^{2}\right)}$. The asymmetry's sensitivity to the strange form factors depends on the kinematics of the experiment. Also, because of the small $1-4 \sin ^{2} \theta_{W}$ factor, there is not much sensitivity to the axial form factor $G_{A}^{Z p}$. The contribution of the other terms depends on the choice of $Q^{2}$ and scattering angle. Since the value of $\epsilon$ becomes larger as $\theta \rightarrow 0$, the sensitivity to $G_{E}^{s}$ is maximized at forward angles.

\subsubsection{The HAPPEx-III Measurement}

Numerous experiments have been conducted to measure this asymmetry at several values of $Q^{2}$ and scattering angle. For the original HAPPEx experiment [44], conducted in 1998-1999, the asymmetry was used to find a linear combination of the strange form factors at $Q^{2}=0.48 \mathrm{GeV}^{2}$. The subsequent second generation HAPPEx$\mathrm{H}$ and HAPPEx-He experiments took place in 2004-2005 and obtained asymmetry measurements at $Q^{2} \approx 0.1 \mathrm{GeV}^{2}$.

Other experiments have been conducted by the A4 collaboration in Mainz [41], SAMPLE at Bates [40] and G0 at Jefferson Labs [46] at this lower momentum transfer of $Q^{2} \approx 0.1 \mathrm{GeV}^{2}$. Figure 1.8 shows the data from all these experiments and the contours represent the most likely values for the strange form factors when taking only the data at this momentum transfer into account.

Figure 1.9 shows the results from all forward-angle scattering experiments that 


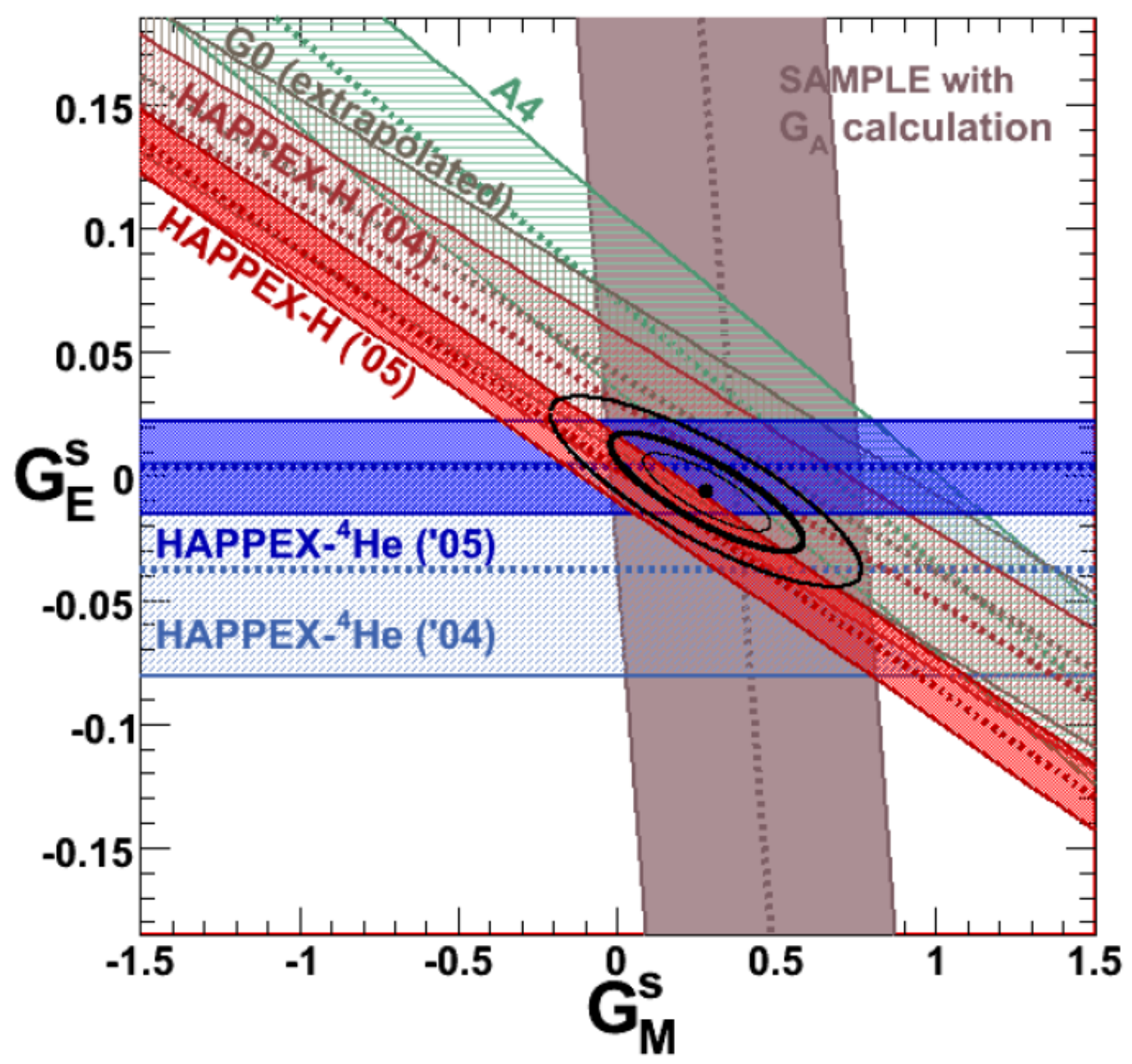

Figure 1.8: Results for the linear combination of strange form factors from several parity violation experiments conducted at $Q^{2} \approx 0.1 \mathrm{GeV}^{2}$. The ellipse is a $95 \%$ confidence region and points are located at the best fit value. 


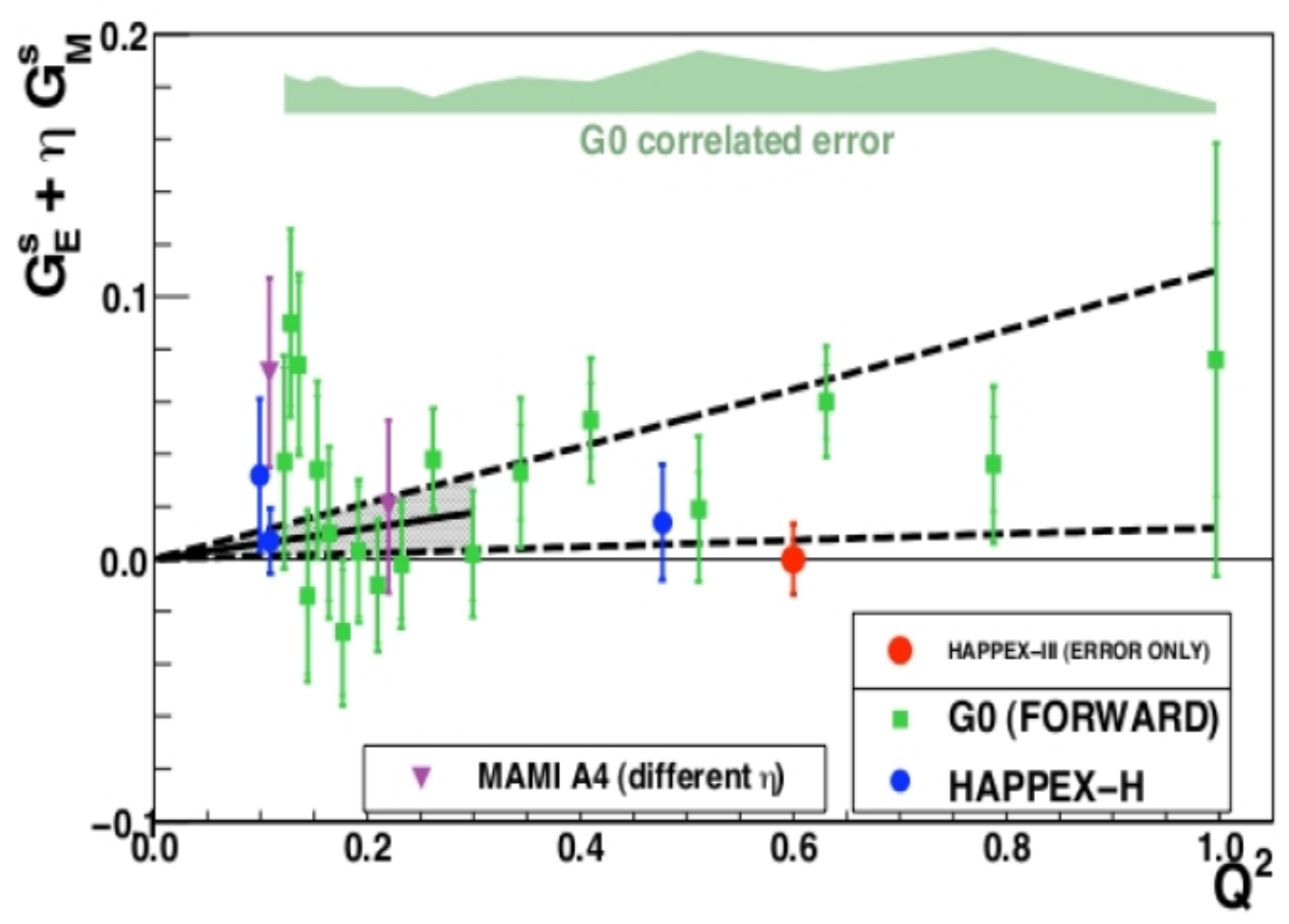

Figure 1.9: Results for HAPPEx, G0 and A4 for the linear combination of strange form factors. The dotted lines show a possible trend with increasing $Q^{2}$. The proposed HAPPEx III error is shown, placed at zero.

have provided constraints on the value of the electric and magnetic strange form factors. The data shown does not rule out a non-zero contribution of the linear combination of strange quark vector form factors to the nucleon form factors. The HAPPEx-III experiment is meant to probe this contribution at a momentum transfer of $Q^{2}=0.6 \mathrm{GeV}^{2}$ with very high precision. The kinematics of this experiment, shown in Table 1.2, were chosen to be sensitive to possibly large non-zero values reported by the G0 collaboration. With such an accurate measurement in that region, a non-zero result would confirm the G0 results and provide evidence for a positive strange form factor contribution. 


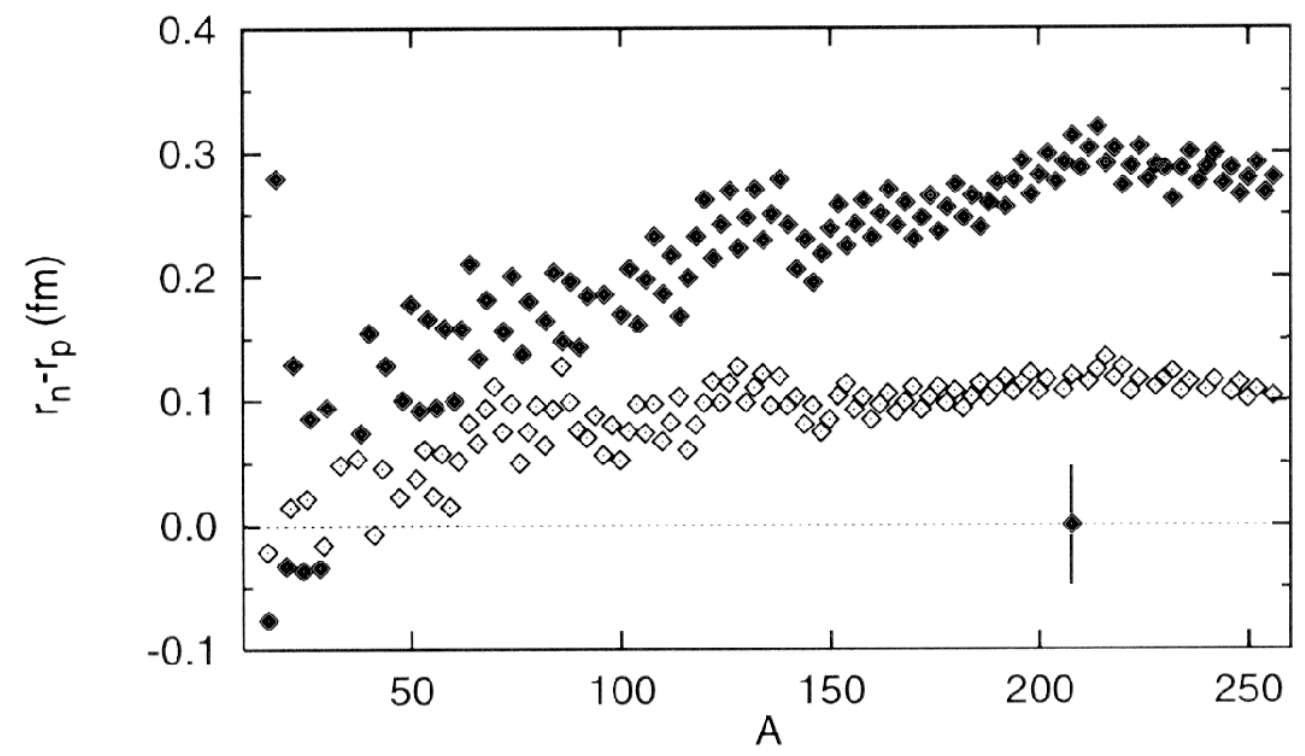

Figure 1.10: Predictions of the difference between proton radius and neutron radius for nuclei with mass number $A$. The black data points represent the relativistic mean field NL1 model and the gray points are for the nonrelativistic Skyrme sk-iii interaction. The data point at $R_{n}-R_{p}$ is included to show the impact of the proposed accuracy of the PREx measurement. The calculations used to make this figure can be found in [12].

\subsection{Neutron RMS Radius of ${ }^{208} \mathrm{~Pb}$}

Heavy nuclei such as ${ }^{208} \mathrm{~Pb}$ have many more neutrons than protons, making it possible for neutrons to form an external layer of a thickness that is not well understood (models suggest skin could range $0-5 \%$ of the nuclear radius). For this reason the current knowledge of nuclear size, obtained through measurements of electromagnetic scattering experiments that only provide information about the proton distribution in the nucleus, needs to be verified to account for the neutron distribution.

According to a paper by Donnelly, Dubach and Sick [9], the techniques involved in parity violation experiments can be useful in measuring the spatial distribution of neutrons in heavy nuclei, and therefore the RMS neutron radius $R_{n}$. This is possible because the $Z^{0}$ boson couples mainly to the neutron at low $Q^{2}$, allowing us to probe the weak charge of the nucleons.

So far, the most accurate values for $R_{n}$ come from various models. Figure 1.10 
shows values for the outer neutron skin thickness $R_{n}-R_{p}$, calculated using the relativistic Skyrme force and a nonrelativistic mean field theory. Included in the plot is a data point at $A=208$ with the proposed error bar of the PREx experiment. A measurement of this value to a high accuracy would be helpful in determining which models are favored. It would also be the first experimental confirmation that there is indeed a neutron skin of measurable extent.

Such an experiment, the Lead Radius Experiment (PREx), took place in the Spring of 2010 at the Thomas Jefferson National Accelerator Facility. PREx measured the parity violating asymmetry in the elastic scattering of polarized electrons from a ${ }^{208} \mathrm{~Pb}$ target. To good approximation, the interaction between an electron and a nucleus can be described by the combination of the electromagnetic vector potential $V(r)$ and the axial potential $A(r)$

$$
\begin{gathered}
V(r)=\int \frac{d^{3} r^{\prime} Z \rho(r)}{|\vec{r}-\vec{r}|}, \\
A(r)=\frac{G_{F}}{2^{2 / 3}}\left[\left(1-4 \sin ^{2} \theta_{W}\right) Z \rho_{p}(r)-N \rho_{n}(r)\right],
\end{gathered}
$$

where $\left.\rho_{(} r\right)$ is the charge density, $\rho_{p}(r)$ is the point proton density and $\rho_{n}(r)$ is the neutron distribution. In the plane-wave Born approximation, the asymmetry becomes

$$
A_{L R}=\frac{-G_{F} Q^{2}}{4 \pi \alpha \sqrt{2}}\left[1-4 \sin ^{2} \theta_{W}-\frac{F_{n}\left(Q^{2}\right)}{F_{p}\left(Q^{2}\right)}\right],
$$

where $G_{F}$ is the Fermi constant, $\alpha$ is the fine structure constant, $F_{p, n}\left(Q^{2}\right)$ are the $Q^{2}$-dependent proton and neutron form factors, respectively

$$
\begin{aligned}
& F_{p}\left(Q^{2}\right)=\frac{1}{4 \pi} \int d^{3} r j_{0}(q r) \rho_{p}(r), \\
& F_{n}\left(Q^{2}\right)=\frac{1}{4 \pi} \int d^{3} r j_{0}(q r) \rho_{n}(r),
\end{aligned}
$$

where $j_{0}$ is the zeroth order spherical Bessel function.

Since the proton form factor is known from other experiments, and the term $\left(1-4 \sin ^{2} \theta_{W}\right)$ is small, Equation 1.33 provides a good measurement of the form factor contribution from the neutrons. From $F_{n}\left(Q^{2}\right)$ one can extract the neutron density after correcting for Coulomb distortions, and therefore make a clean measurement of the RMS neutron radius $R_{n}$, as seen in Figure 1.11 [10]. The PREx result also has implications on atomic physics and astrophysics. 


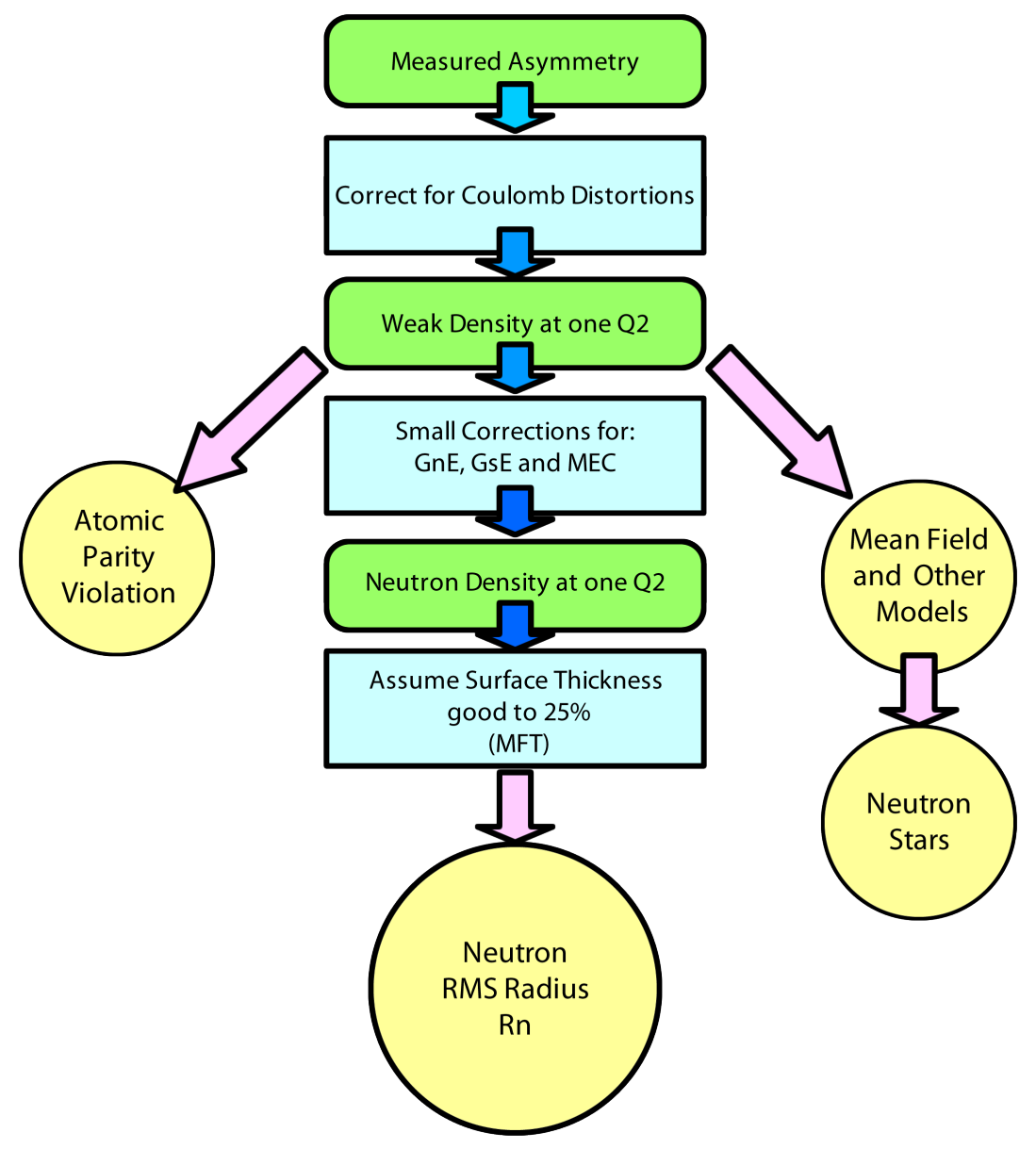

Figure 1.11: Flow chart of the physics data analysis of a neutron radius experiment. It shows how one can find a value for the RMS neutron of ${ }^{208} \mathrm{~Pb}$ by using a measurement of the neutron form factor. 


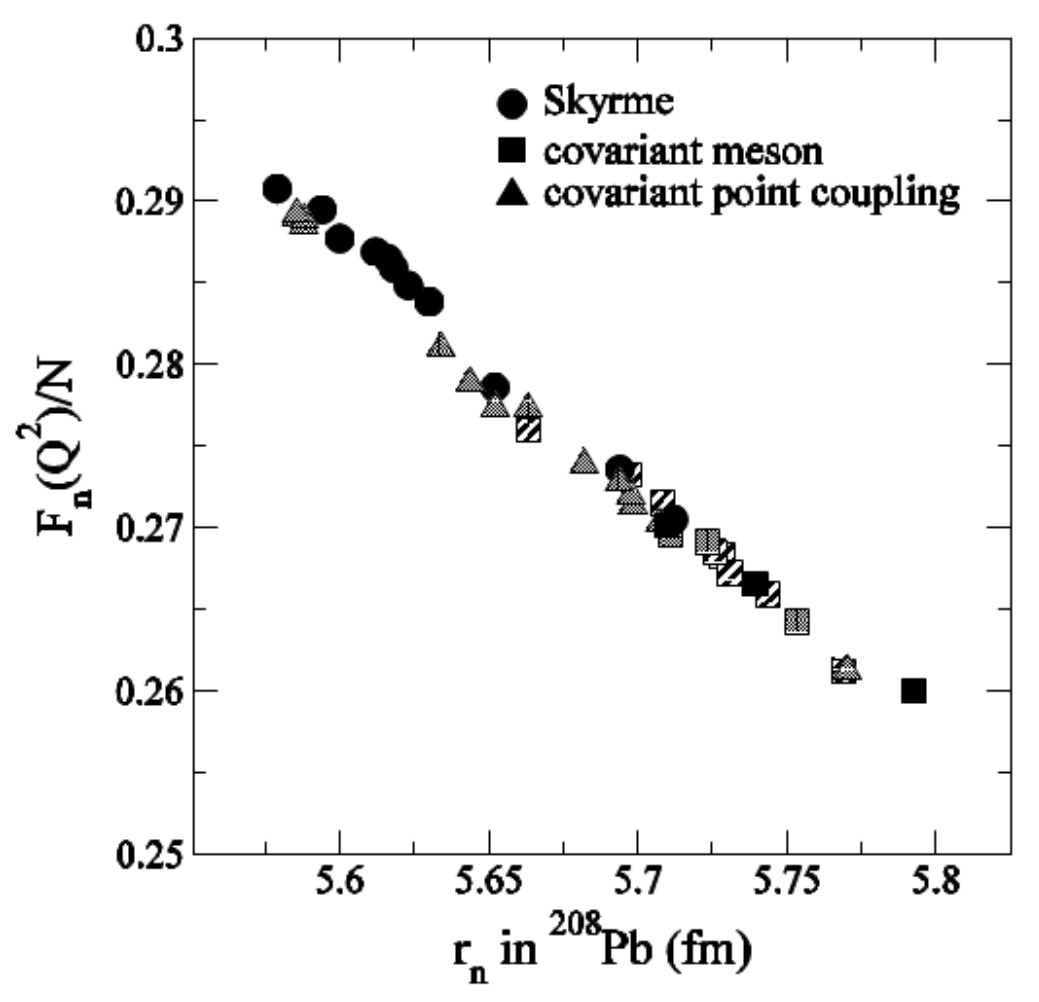

Figure 1.12: Neutron form factor for ${ }^{208} \mathrm{~Pb}$ at the momentum transfer of the PREx experiment vs. the RMS neutron radius of ${ }^{208} \mathrm{~Pb}$ for a variety of models [13].

The measurement of $R_{n}$ can provide contraints on the equation of state (EOS), which describes the pressure of neutron matter as a function of density. There is also a strong correlation between this value and the radius of a neutron star that depends on the structure of the EOS. This relationship helps us understand the symmetry energy $S$, a value that determines how quickly a neutron star cools [11].

Figure 1.12 shows predictions for the value of the neutron radius for numerous mean field theory (MFT) models. The linear correlation to the value of the neutron form factor makes it possible for the PREx experiment to find a value for the neutron radius by measuring the parity violating asymmetry at just one value of four-momentum transfer.

Many challenges were encountered while planning and running the experiment. The asymmetry being measured is at the half ppm level, which requires a very high level of presicion. Since the goal of the experiment was to measure $R_{n}$ to $1 \%$ accuracy, the asymmetry must be measured to within $3 \%$. In order to achieve this, all sources 
of error must be minimized. Some of these include false asymmetries associated with helicity correlation of certain beam parameters, pedestal noise of the electronics setup and instabilities in the target temperature and density. Also, several new components were installed in the beam line and Hall A to make this experiment possible. These developments will be discussed in detail in Chapter 3 of this thesis. 


\section{CHAPTER 2}

\section{EXPERIMENTAL DESIGN}

The HAPPEx collaboration aimed to measure the parity violating asymmetry at a large value of four-momentum transfer with unprecedented accuracy. This was done with a longitudinally polarized beam of electrons produced by the Continuous Electron Beam Accelerator Facility (CEBAF) at Jefferson Labs. By detecting the elastic electrons scattered off a liquid hydrogen target at an angle of $\theta \approx 13.7^{\circ}$, an asymmetry can be calculated.

This measurement, conducted at a four-momentum transfer squared of $Q^{2} \approx 0.62$ $\mathrm{GeV}^{2}$ and beam energy $E_{b}=3.49 \mathrm{GeV}$, will help constrain the positive trend of strange quark contributions to the proton form factors, as discussed in Section 1.6.3. Figure 2.1 shows a schematic of all the components used to implement the experiment with emphasis on Hall A, where it took place. The design of the experiment was dependent on the ability to measure this parts per million (ppm) asymmetry with a small statistical error of $\approx 3 \%$. The methods used to accomplish this will be discussed in this chapter.

\subsection{Experimental Technique}

The goal of many parity violating electron scattering experiments is to measure a very small asymmetry, dependent on the difference over sum of the cross-sections between the scattering of left- and right-handed longitudinally polarized electrons

$$
A_{d e t}=\frac{\sigma_{R}-\sigma_{L}}{\sigma_{R}+\sigma_{L}} .
$$

Electrons polarized parallel to the beam momentum are said to have a right-handed helicity. Those directed anti-parallel to the beam therefore have left-handed helicity. For the HAPPEx experiments, electrons were scattered off unpolarized cryogenic targets. 


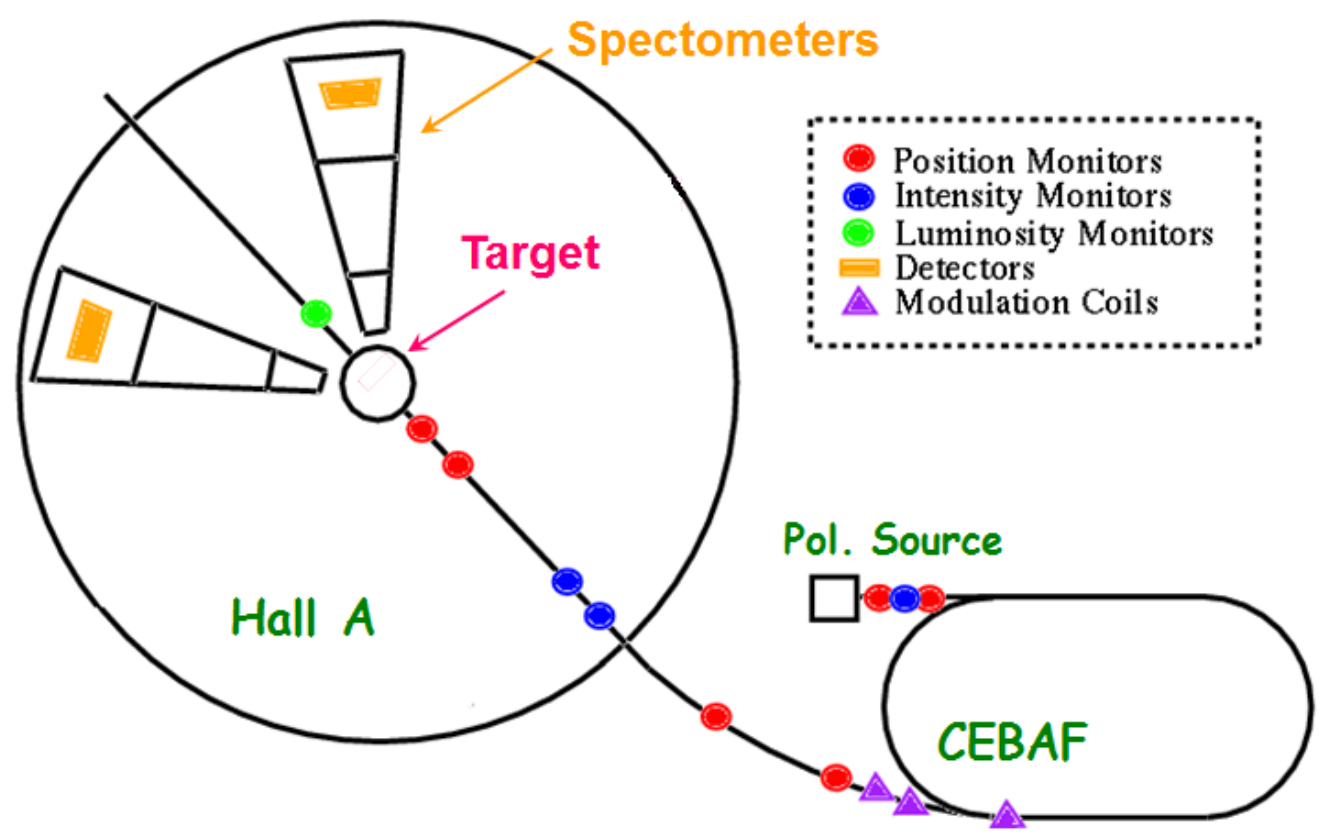

Figure 2.1: The layout of the entire HAPPEx experiment, emphasizing the components along the beamline and inside Hall A.

In order to get an accurate measurement of the asymmetry, several techniques were employed to control and monitor any false asymmetries. The first of these was to ensure proper calibration of the laser and alignment of the source optics used to create the electron beam. This can be done by adjusting the wavelength of the laser light in order to maximize its longitudinal polarization and minimize helicitycorrelated variations of the beam.

Beam properties such as current, position and energy were measured at several locations along the beam line to ensure beam quality and stability. The polarized beam of electrons could also be perturbed intentionally with several magnetic steering coils. This technique was useful in determining how small changes in the beam properties affect the asymmetry measurement.

After accounting for these helicity-correlated false asymmetries, the most important and biggest correction to be made is for the degree of beam polarization. Therefore, two techniques were employed to monitor the beam polarization throughout data collection. These polarimeters are able to measure the longitudinal beam polarization with an accuracy better than $2 \%$. 
The helicity of the beam for the HAPPEx-III experiment was switched between left and right at a rate of $30 \mathrm{~Hz}$ in a pseudo-random fashion, with each state followed by its complement. This allowed for a measurement of the asymmetry at a rate of $15 \mathrm{~Hz}$. This rapid reversal rate is helpful in reducing any systematic errors caused by slow drifts in the properties of the beam. The experiment used integrating detectors to collect the scattered flux during each helicity state, which is digitized and recorded by a data acquisition system. This allows us to calculate independent asymmetry measurements for each adjacent helicity pair. HAPPEx-III was able to collect enough data to accomplish a $3.3 \%$ statistical error and systematics were controlled to the $<2 \%$ level.

\subsubsection{Interpreting our Asymmetry Measurement}

The asymmetry being measured in the HAPPEx experiments depends on the crosssection of electron-proton scattering. It is sufficient to measure a quantity proportional to this cross-section since any common factors will cancel out. This is done by integrating the scattered flux $D$ and using measurements of the beam intensity $I$ to normalize the signal in each helicity window, so that Equation 2.1 becomes

$$
A_{\text {det }}=\frac{D_{R} / I_{R}-D_{L} / I_{L}}{D_{R} / I_{R}+D_{L} / I_{R}}=\frac{\Delta S}{2 S}
$$

so that $S_{R, L}=D_{R, L} / I_{R, L}$ and $\Delta S=S_{R}-S_{L}$.

Ideally, the statistical error of this measurement is due to the counting statistics of the experiment, so that the error for a single pair is

$$
\epsilon=\frac{1}{\sqrt{N_{R}+N_{L}}}
$$

where $N_{R}$ and $N_{L}$ are the number of right- and left-handed electrons detected in each helicity pulse. When studying the distributions of the pair data, counting statistics gives the minimum width that can be obtained. Other sources of noise, such as fluctuations in the target density, can broaden the distribution of pairs by adding in quadrature with counting statistics. Therefore, these contributions must be minimized during the experiment to achieve the statistical goals in the allotted running time.

We must also consider any systematic errors arising from cross-section measurements. The two main sources of error can be described as a common-mode offset 
$\Delta \sigma_{C M}$ and a helicity-correlated offset $\Delta \sigma_{H C}$

$$
A_{d e t}=\frac{\left(\sigma_{R}+\Delta \sigma_{C M}+\Delta \sigma_{H C}\right)-\left(\sigma_{L}+\Delta \sigma_{C M}-\Delta \sigma_{H C}\right)}{\left(\sigma_{R}+\Delta \sigma_{C M}+\Delta \sigma_{H C}\right)+\left(\sigma_{L}+\Delta \sigma_{C M}-\Delta \sigma_{H C}\right)} .
$$

Errors due to common-mode offsets can be caused by slow drifts in the PMT gain or fluctuations in beam parameters, but do not depend on the helicity of the beam. Assuming that $\Delta \sigma_{H C} \ll \sigma_{R(L)}$ and defining $\sigma \equiv\left(\sigma_{R}+\sigma_{L}\right) / 2$, Equation 2.4 can be simplified to show that the common-mode offset produces an error that is proportional to the true asymmetry,

$$
A_{\text {det }}=A_{\text {true }}\left(1-\frac{\Delta \sigma_{C M}}{\sigma}\right)+\frac{\Delta \sigma_{H C}}{\sigma} .
$$

This means that a $1 \%$ error in the measured flux contributes the same $1 \%$ systematic error to the asymmetry. This makes the asymmetry measurement insensitive to common-mode offsets. Any helicity-correlated offsets will not depend on the size of the asymmetry and must be monitored closely. One important goal of the experiment was to control these errors so that they were much lower than the expected statistical error.

\subsubsection{False Asymmetries and Other Corrections}

Small variations in several beam parameters can affect the amount of flux that reaches the detectors. During the experiment, such helicity-correlated fluctuations were monitored so that any contributions to the measured asymmetry could be corrected for during data analysis. Changes in beam position, intensity and energy create false asymmetries that can be corrected for by using Equation 2.6, where $S$ is the detector flux $D$ divided by the intensity $I, E$ is the beam energy, $\Delta x_{i}$ are position differences, and $\alpha_{E, i}$ are correlation slopes.

$$
A_{\text {corr }}=\frac{\Delta S}{2 S}+\alpha_{E} \frac{\Delta E}{2 E}+\Sigma_{i} \alpha_{i} \Delta x_{i} .
$$

These helicity-correlated false asymmetries correspond to the last term of Equation 2.5 and are a very important contribution to the experiment's systematic error. Two independent techniques, linear regression and beam modulation, are used to make the necessary corrections. Once that is done, $A_{\text {corr }}$ must be adjusted for the degree of beam polarization and backgrounds. 


\subsubsection{Backgrounds}

Once the measured detector asymmetry has been corrected for all helicity-correlated false contributions, it is time to consider backgrounds and dilution factors. Even thought the experiment is designed to minimize backgrounds such as inelastically scattered electrons, any small background fraction $f_{i}$ can affect the asymmetry

$$
A_{\text {phys }}=\frac{K}{P_{b}} \frac{A_{\text {corr }}-P_{b} \Sigma_{i} f_{i} A_{i}}{1-\Sigma_{i} f_{i}}
$$

The $A_{i}$ values are the flux-weighed asymmetries of the background processes. For HAPPEx-III, the main sources of background come from the aluminum end-caps of the target cell and particles that re-scatter off the spectrometer walls. The degree of beam polarization $P_{b}$ must also be considered and was carefully measured throughout the experiment using two different techniques.

\subsection{Polarized Beam}

The setup used to produce polarized electrons was designed to minimize any helicity correlated beam systematics [16]. The process begins by creating the right kind of longitudinally polarized laser light $(\lambda=780 \mathrm{~nm})$ with an elaborate optics setup. Each experimental hall has a $499 \mathrm{MHz}$ diode laser which can be optimized independently for their specific needs. This light then passes through a Pockels cell (see Figure 2.2), used to change the linear polarization to left or right circular polarization. The Pockels cell is also designed to allow for rapid helicity flips that help cancel out effects from slow drifts in beam properties.

The circularly polarized light can also pass through an Insertable Half-Wave Plate (IHWP), which reverses the polarization of the beam. This technique is used to cancel possible systematic effects that cannot be accounted for by doing rapid helicity flips. All three lasers are used to create a $1497 \mathrm{MHz}$ bunch that illuminates the surface of a strained 'superlattice' GaAs photocathode. Photons are absorbed by the photocathode and electrons are excited from the valence band to the conduction band (see Figure 2.3). Finally, a potential difference is applied that strips away longitudinally polarized electrons and draws them into the accelerator, creating a continuous wave (CW) electron beam. 


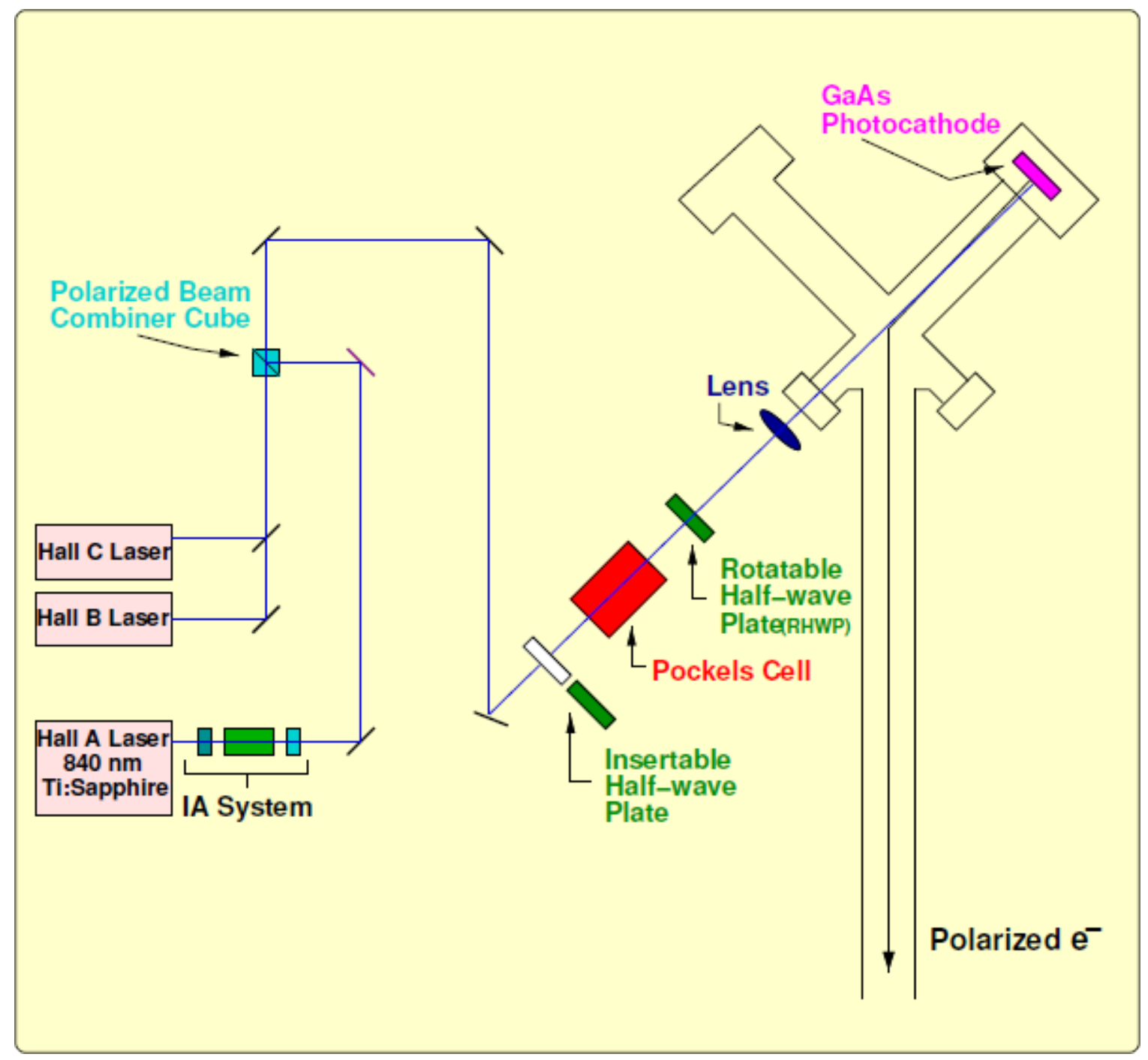

Figure 2.2: Schematic of source setup shows how the Hall A laser gets used to create a polarized beam of electrons [57]. A similar setup was also used for the previous HAPPEx experiments. 


\subsubsection{Pockels Cell}

The Pockels cell is one of the last optical components the polarized light goes through before reaching the GaAs photocathode. It is composed of a variable-wave plate and provides the fast reversal of the electron-beam helicity [16]. When longitudinally polarized laser light goes through, it comes out circularly polarized. This is achieved by applying a very large voltage of $\pm 2.5 \mathrm{kV}$ and rapidly changing the sign of this voltage. The sign of the voltage determines whether the Pockels cell behaves like a positive or negative quarter-wave plate allowing for electrons with left- or righthanded helicity to be produced by the GaAs crystal. The applied voltages must be set carefully to maximize the polarization of the light. Accurate determination of the proper voltage settings will make sure that helicity-correlated false asymmetries are minimized.

\subsubsection{Insertable Half-Wave Plate}

The rapid helicity flips achieved by the Pockels cell only minimize sensitivity to slow drifts. In fact, the rapid flip of the sign of the voltage might introduce other systematic errors via cross-talk with the Data Acquisition (DAQ) electronics that can be caused by ground loops. These and other helicity-correlated false asymmetries can be canceled out by introducing a slow helicity reversal technique.

This can be achieved by using an Insertable Half-wave plate (IHWP) as part of the source optics. This is referred to as a passive flip because it does not affect the magnitude of the measured asymmetry, only its sign. Also, since the DAQ does not 'know' about the flip, it helps cancel out certain false asymmetries. During the experiment, the state of the IHWP was nominally changed after a full day of good data taking, or after collecting one million good asymmetry pairs.

\subsubsection{GaAs Photocathode}

When circularly polarized photons hit the Gallium Arsenide cathode, a longitudinally polarized beam of electrons is produced through photo-emission. This happens when photons of a specific helicity excite electrons from the valence band $\left(P_{3 / 2}, m_{j}= \pm 3 / 2\right)$ to the conduction band $\left(S_{1 / 2}= \pm 1 / 2\right)$. When illuminating the cathode with laser light of the proper wavelength, it is possible to create a nearly $100 \%$ polarized beam of 


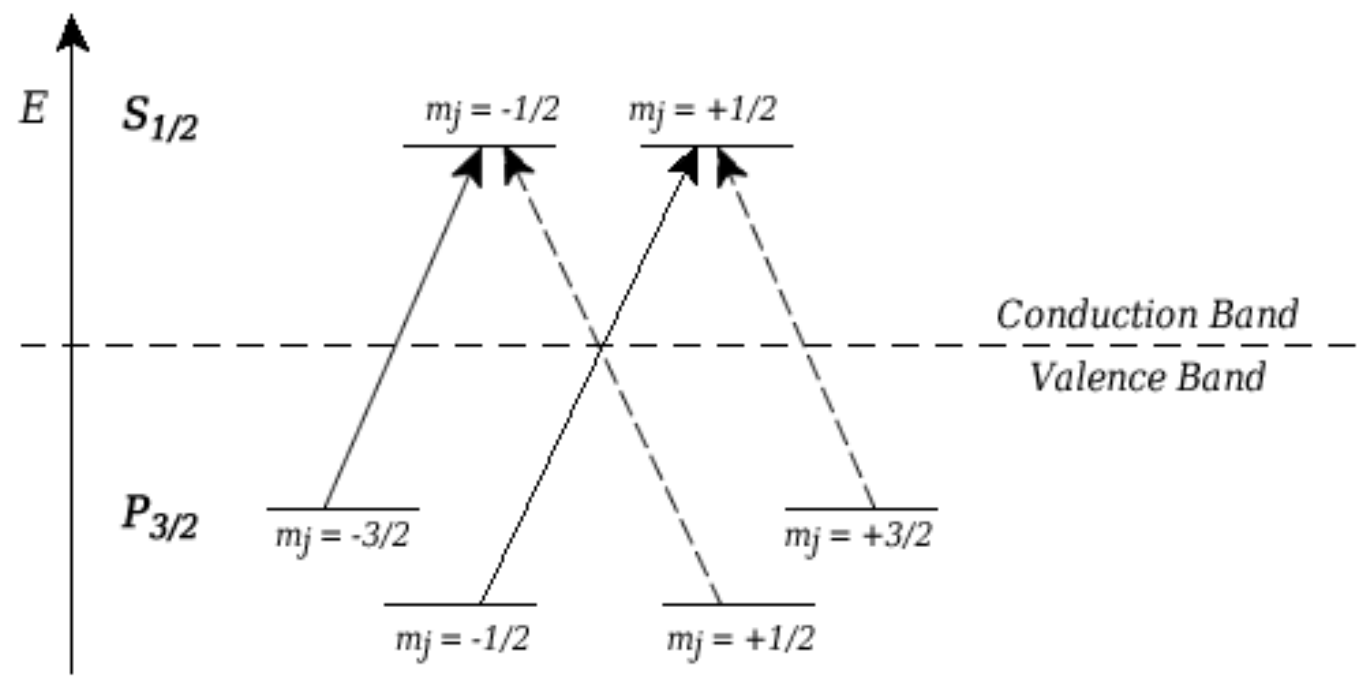

Figure 2.3: Diagram of the allowed energy transitions for Gallium Arsenide

electrons, a feat which is very challenging to accomplish. Figure 2.3 shows a diagram of the allowed energy level transitions, with solid and slashed lines representing the different helicity states.

Since the original HAPPEx experiment, changes have been made to the cathode and laser optics in order to maximize the polarization of the excited electrons. The GaAs crystal is said to be a 'superlattice' cathode and is made up by alternating thin layers of GaAs and GaAsP grown on a thick layer of GaAsP [17]. The 'superlattice' cathode makes it possible to maximize the degree of polarization by breaking the degeneracy of the $P_{3 / 2}$ levels. This allows for the transition from $\left(P_{3 / 2}, m_{j}= \pm 1 / 2\right)$ to be suppressed when the laser has the correct wavelength.

\subsection{Accelerator}

The HAPPEx-III experiment took place during the Fall of 2009 at the Thomas Jefferson National Accelerator Facility (TJNAF) in Newport News, Virginia. This facility is funded by the U.S. Department of Energy's Office of Science and is used by scientists worldwide to conduct research related to the structure of the nucleus. To do this, the Continuous Electron Beam Accelerator Facility (CEBAF) sends beam to 


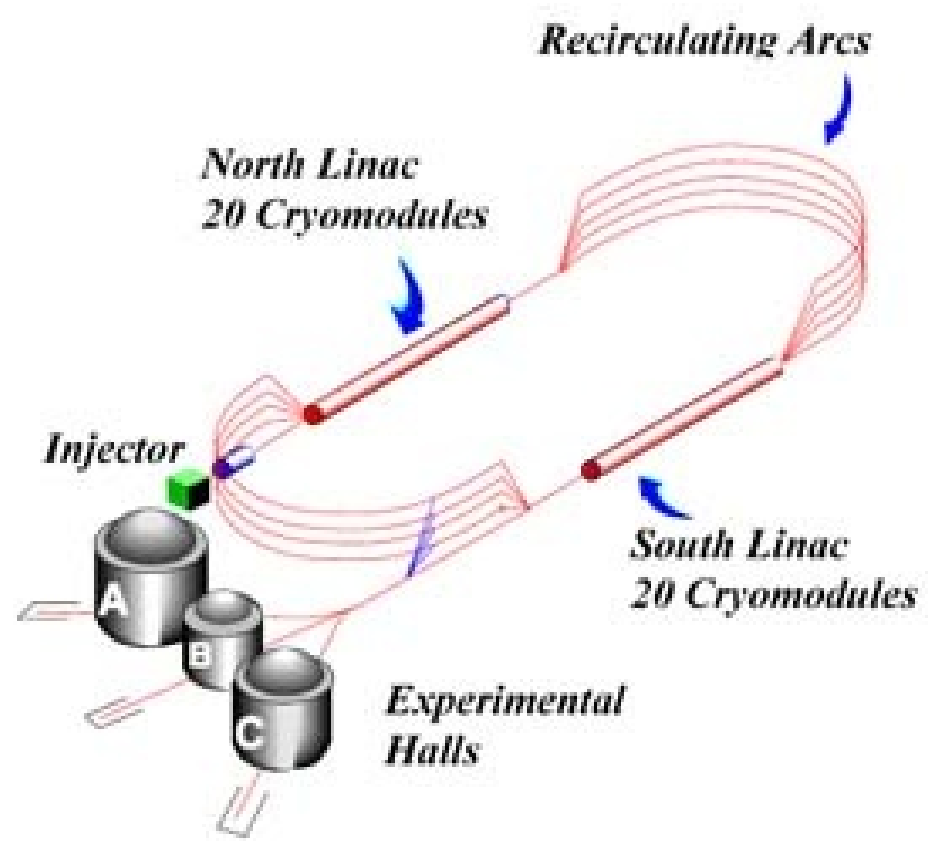

Figure 2.4: Layout of CEBAF shows the race-track path taken by the electron beam, made up of two linacs and five recirculating arcs connecting them at each end.

three experimental halls: A, B and C (see Figure 2.4).

The accelerator itself consists of a series of superconducting radio frequency (SRF) accelerating cavities distributed along two linear accelerator sections, or linacs. The two linacs are connected by recirculating arcs, which bend the beam's trajectory by $180^{\circ}$ so that it follows a racetrack path. The beam can be recirculated up to five times, producing a maximum beam energy of $6 \mathrm{GeV}$ and a beam current of up to 200 $\mu \mathrm{A}$. Once the proper energy is established, the beam can be delivered to one or all of the experimental halls concurrently. The beam energy can be adjusted depending on each hall's beam requirements. The lab is currently working on upgrades that will make it possible to reach beam energies up to $12 \mathrm{GeV}$.

The original HAPPEx experiment, conducted in 1998-99, measured the parity violating asymmetry at $Q^{2} \approx 0.48 \mathrm{GeV}^{2}$ with a beam energy of $E=3.36 \mathrm{GeV}$. HAPPEx-III made a similar measurement at a slightly higher momentum transfer of $Q^{2} \approx 0.62 \mathrm{GeV}^{2}$ and beam energy $E=3.49 \mathrm{GeV}$. This new experiment took advantage of recent developments in the polarization and increased quality of the beam systematics. While the polarization of the beam was about $35-70 \%$ for HAPPEx, for 


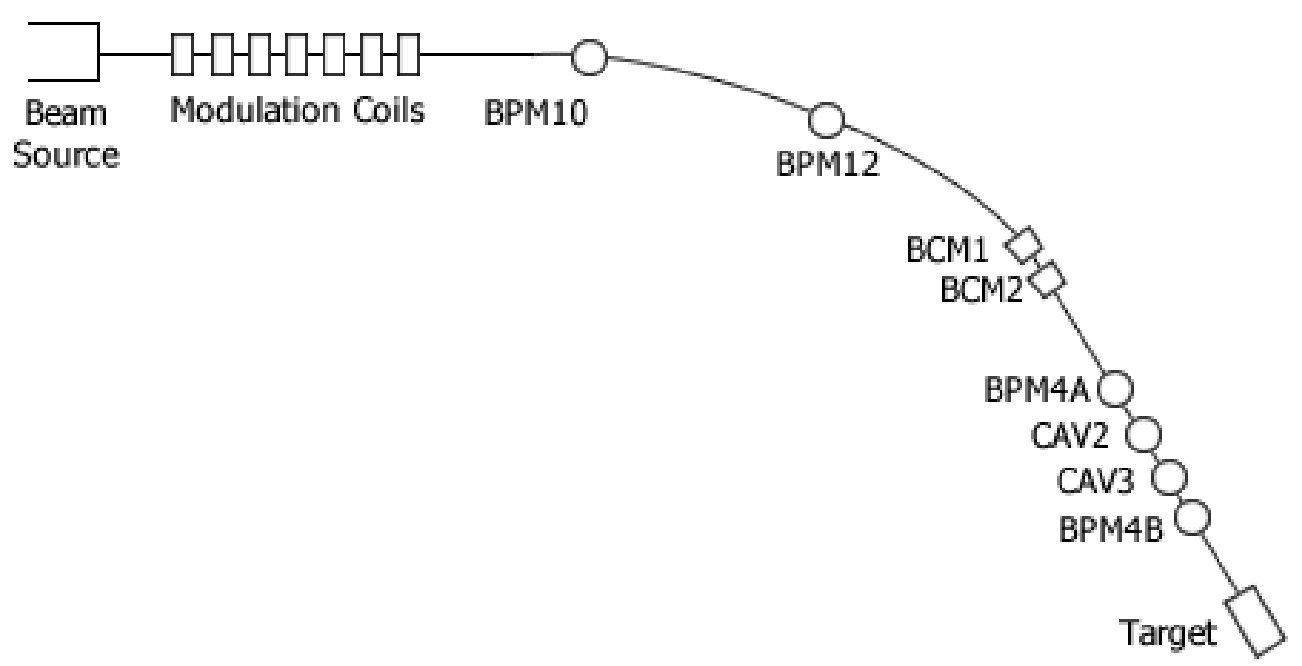

Figure 2.5: Schematic of Hall A beamline shows location of important beam monitors and modulation coils.

HAPPEx-III it was able to reach a value of $\approx 89.4 \%$, allowing for a very high-precision measurement in a short time.

\subsection{Beamline Components}

Once the electron beam is tuned to match the kinematics of the experiment, it must be delivered to each Hall through it's own beamline. There are numerous monitoring systems used along the beamline to keep track of beam properties such as position and energy, some of which are shown in Figure 2.5. There are also several modulation coils used to purposely perturb the beam properties. This technique is helpful in understanding how such changes affect the data collected by the detectors in the Hall.

\subsubsection{Current Monitors}

When calculating the experimental asymmetry, we require the detector signals to be normalized by the beam intensity (see Equation 2.2). For this reason, several beam current monitors (BCMs) are used along the beam line to make this measurement. The two main BCMs are RF resonance cavities located $25 \mathrm{~m}$ upstream of the tar- 


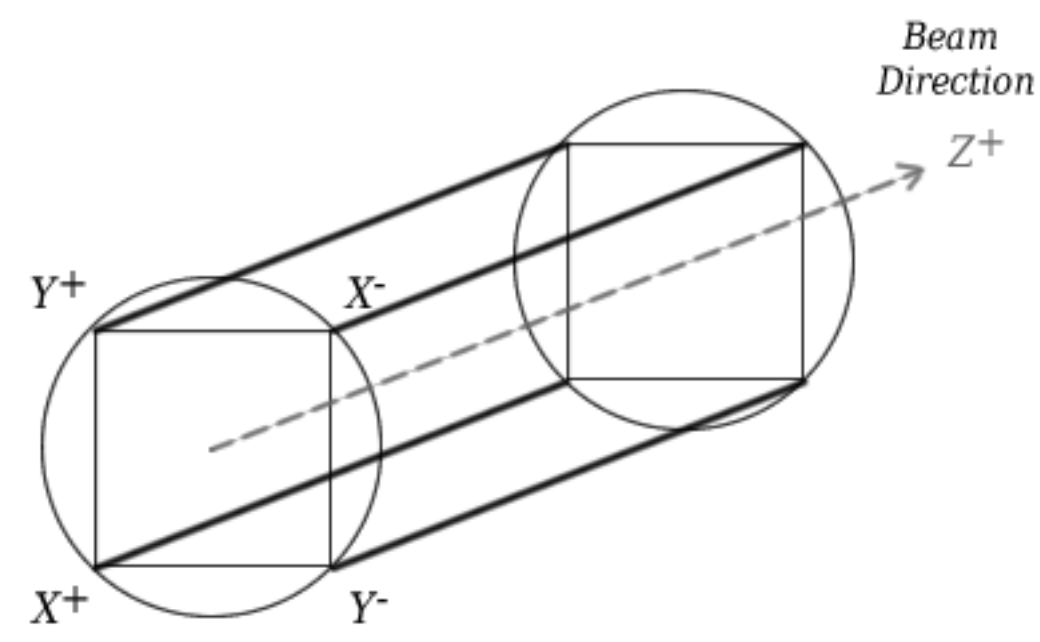

Figure 2.6: The Stripline Beam Position Monitors are composed of four wires located symmetrically around the beamline, labeled as the Z-axis.

get. They output a signal proportional to the beam intensity when tuned to the accelerator's frequency of $1497 \mathrm{MHz}$ [15].

An Unser current monitor was also used to calibrate the linearity of the cavity monitors. The Unser is a Parametric Current Transformer and provides an absolute current reference [19]. It is enclosed in a temperature controlled box to provide good magnetic shielding and help avoid signal drifts. While the Unser signal is much noisier and has a slow drift over time, it is known to be linear down to zero current, whereas the cavity monitors become nonlinear at signals lower than a few $\mu \mathrm{A}$. All these signals were read out by voltage-mode ADCs in the integrating DAQ. The RF cavity signals were additionally read out after being amplified by factors of three and ten to take advantage of the dynamic range of the ADCs.

\subsubsection{Position Monitors}

Between the accelerator and the target in Hall A, there are several beam position monitors (BPMs) used to measure any helicity correlated differences in position, angle and energy (see Figure 2.5). Stripline BPMs [18] consist of four wire antennae oriented parallel to the beam direction and placed symmetrically around the beamline (see 
Figure 2.6). The antennae are rotated $45^{\circ}$ with respect to the Hall A coordinate system. Each wire produces a radio frequency which is processed by electronics to create a DC voltage signal proportional to the beam current and position.

The beam position is found to be the difference over the sum of opposing wires multiplied by a factor related to the distance between wires

$$
X^{\prime}=\frac{\kappa}{2}\left(\frac{X^{+}-X^{-}}{X^{+}+X^{-}}\right), Y^{\prime}=\frac{\kappa}{2}\left(\frac{Y^{+}-Y^{-}}{Y^{+}+Y^{-}}\right) .
$$

This must then be rotated by $45^{\circ}$ to find the actual beam position in the lab coordinate system

$$
\left(\begin{array}{l}
X \\
Y
\end{array}\right)=\left(\begin{array}{rr}
\cos \left(45^{\circ}\right) & -\sin \left(45^{\circ}\right) \\
\sin \left(45^{\circ}\right) & \cos \left(45^{\circ}\right)
\end{array}\right)\left(\begin{array}{l}
X^{\prime} \\
Y^{\prime}
\end{array}\right) .
$$

The HAPPEx-III experiment mainly used two BPMs located near the target and another located where the beamline bends toward Hall A. BPMs 4A and 4B are both located upstream of the target, about $6 \mathrm{~m}$ and $1 \mathrm{~m}$ away, respectively. BPM12 is located in a high dispersion point between bending magnets, providing a position difference that is sensitive to beam energy. BPM12 was used to monitor any helicitycorrelated fluctuations in the beam energy.

\subsubsection{Modulation Coils}

The cross-section of the elastic electron-proton interaction depends on the beam energy and the scattering angle. These quantities must be measured since any small changes can introduce false components to the physics asymmetry. In order to understand the sensitivity of our measurements to these small helicity-correlated parameters, we use a technique called beam modulation (also known as 'dithering'). For this purpose, seven modulation coils are installed along the Hall A beam line, several meters upstream of the main bend.

Four of the coils modulate the beam horizontally while the rest do so vertically. An energy vernier is also used at the end of the accelerator's south linac to modulate the beam energy. Dedicated studies were conducted during production running to make sure that this energy modulation did not affect the other experimental halls negatively. The modulation technique is non-invasive and can run throughout the entire data taking process. 

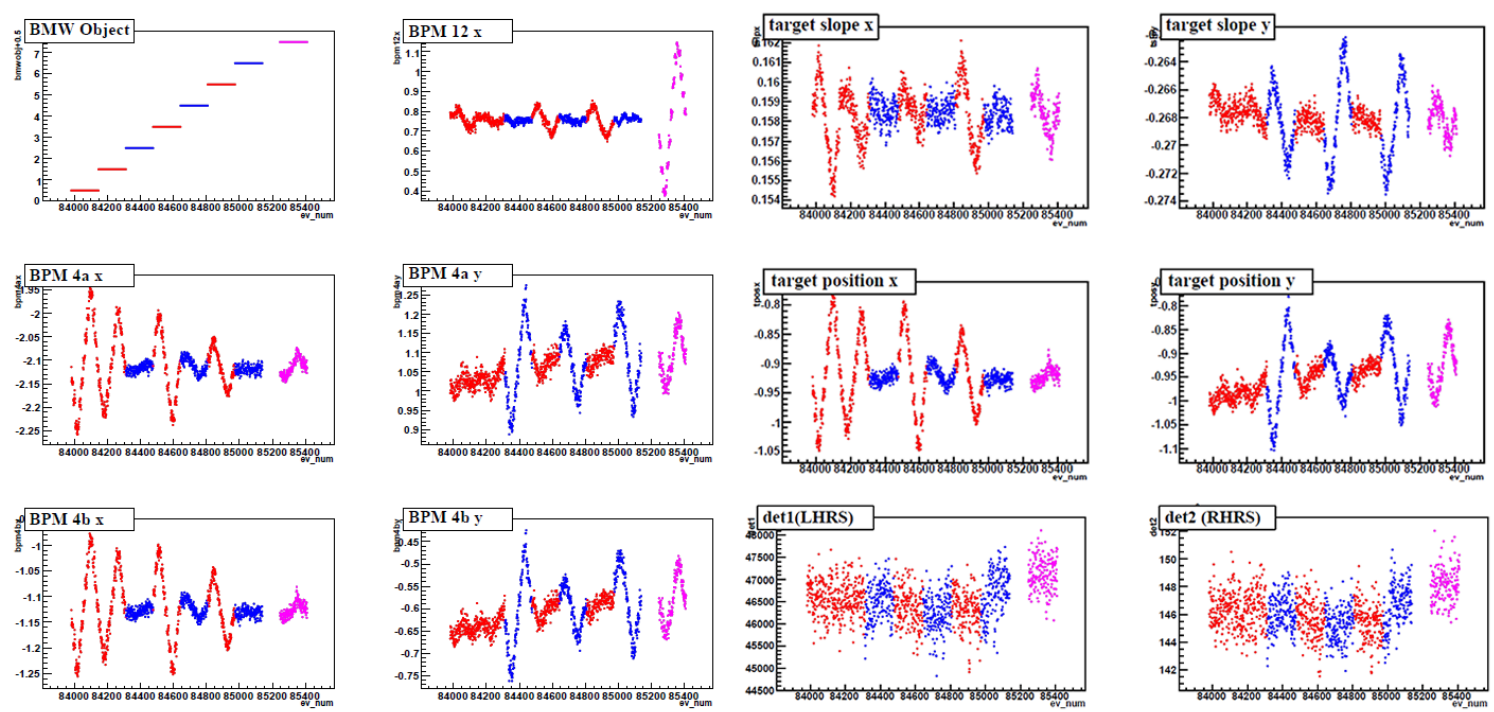

Figure 2.7: Sample plots of beam modulation response. Red and blue data represent horizontal and vertical modulation, accordingly. The magenta data is energy modulation. These plots are representative of one 'dithering' cycle.

During a 'dithering' cycle, we analyze how our detectors are affected by each modulation. Figure 2.7 shows how the BPM signals vary during one of these cycles Eventually, the raw asymmetry will be corrected by using any correlations between the measured flux and the modulated beam parameters. These correlations are calculated by doing a simple matrix inversion, as will be discussed in Section 4.4.1.

\subsubsection{Raster}

The high intensity beam produced by the accelerator, with an intrinsic beam spot size of about $100 \mu \mathrm{m}$, can cause local heating of the target. In turn, this can create density fluctuations inside the target that contribute nonstatistical noise to the asymmetry measurement. This local heating could also compromise the thin aluminum end-caps of the target cell. To reduce these effects, the beam is swept over a small rectangular area by a fast rastering system.

The raster is composed of a pair of horizontal and vertical dipole magnets located $23 \mathrm{~m}$ upstream of the target. The magnets are driven by a triangular wave pattern and have practically no dwell time at the peaks. During the experiment, tests are conducted to establish the raster size that will minimize any noise contributions from 


\begin{tabular}{c|c}
\hline \hline Target & Description \\
\hline Loop 1 Cell & $20 \mathrm{~cm} \mathrm{D2}$ \\
\hline Loop 2 Cell & $20 \mathrm{~cm} \mathrm{H} 2$ \\
\hline Loop 3 Cell & $25 \mathrm{~cm} \mathrm{H} 2$ \\
\hline Optics & Carbon foils $0, \pm 7.5, \pm 15 \mathrm{~cm}$ \\
\hline Dummy Hole & Al foils $2 \mathrm{~mm} \mathrm{hole}$ \\
\hline Dummy target & Al foils $\pm 12.5 \mathrm{~cm}$ \\
\hline Carbon Hole & Carbon foil $2 \mathrm{~mm}$ hole \\
\hline Thin Ta & Tantalum foil \\
\hline Thick Ta & Tantalum foil \\
\hline BeO & BeO \\
\hline \hline
\end{tabular}

Table 2.1: Details about targets installed during the HAPPEx-III experiment. More details about the target configuration can be found in [22].

target density fluctuations, also referred to as 'target boiling.' For the HAPPEx-III experiment, the raster size used was $3.5 \mathrm{~mm}$ by $4.5 \mathrm{~mm}$.

\subsection{Target Ladder}

The target ladder assembly is part of the standard equipment used in Hall A. It consists of three cryogenic loops and a number of solid targets used for optics calibration and background measurements. The assembly is housed inside a scattering chamber along with sub-systems for cooling, temperature and pressure monitoring. The chamber is maintained at a $10^{-6}$ torr vacuum, coupled to the beam pipe and spectrometers. Table 2.1 shows a summary of the targets used during the HAPPEx-III experiment.

The target installed on loop 3, a $25 \mathrm{~cm}$ long, unpolarized, liquid hydrogen cryotarget, was the main target used during data collection. There were two other cryotargets installed. A $20 \mathrm{~cm}$ long liquid deuterium target used for the PVDIS experiment, and a $20 \mathrm{~cm}$ long liquid hydrogen cell, installed as a backup. The target cells are made of Aluminum and are $2 \mathrm{~cm}$ in diameter. An Aluminum dummy target composed of two Aluminum foils located at $\pm 12.5 \mathrm{~cm}$ from the nominal target center was installed to measure backgrounds from the entrance and exit windows of the hydrogen cell. The 


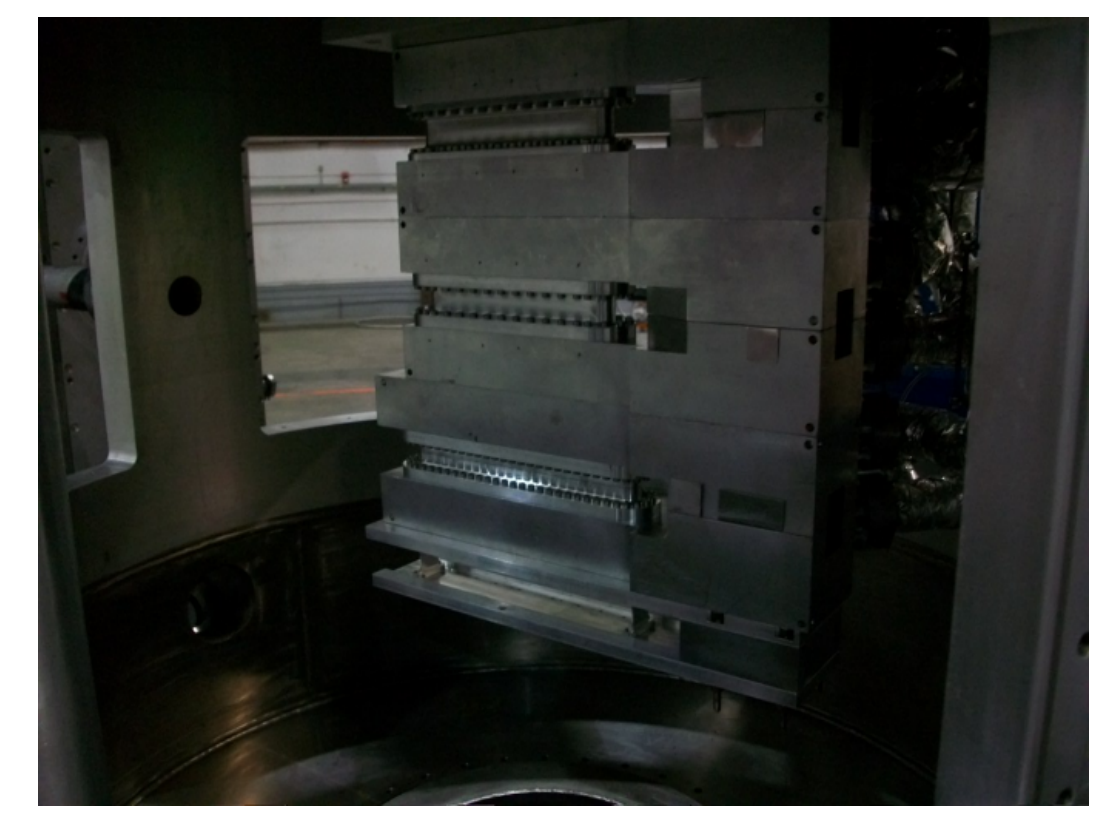

Figure 2.8: Picture of the cryogenic target ladder inside the scattering chamber.

solid targets include Carbon, BeO and two different thicknesses of Tantalum.

\subsection{Hall A High Resolution Spectrometers}

The experimental hall has a set of identical magnetic spectrometers used to double the acceptance of the experiment. These High Resolution Spectrometers [15] were designed to focus and guide particles of interest toward specially designed detector packages. They are each made up of a QQDQ (Q for quadrupole and D for dipole) arrangement of superconducting magnets, symmetrically placed on each side of the beamline. Two main advantages of their symmetry is: 1) to double the amount of counting statistics, and 2) to cancel out any left-right helicity correlated asymmetries. The dimensions and locations of each magnet are shown in detail in Figure 2.9.

The spectrometers have a 5.5-msr acceptance each and are capable of a momentum resolution at the $10^{-4}$ level for a momentum range between $0.8-4.0 \mathrm{GeV}$. The particles that go into the first two superconducting quadrupoles are focused toward a $45^{\circ}$ vertical bending dipole and finally go through one last focusing quadrupole. Settings for all magnets are determined so that the elastically scattered electrons are well separated from inelastic backgrounds and guided toward the focal plane of the 


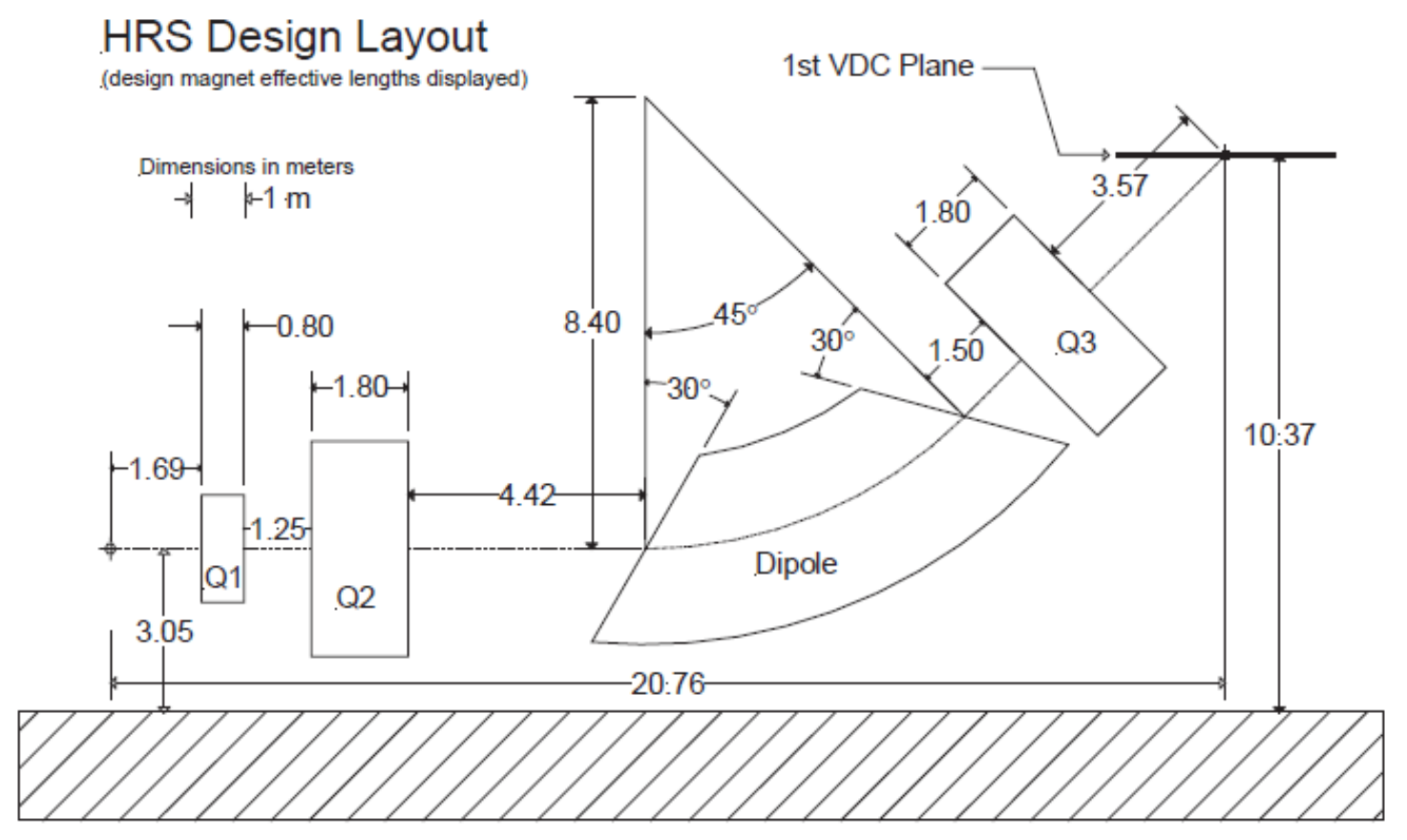

Figure 2.9: Detailed diagram of the magnetic components that make up each High Resolution Spectrometer [15].

detectors, located at the top of the spectrometers.

Because of the size of the spectrometers, the minimum angle at which they can be positioned is $12.5^{\circ}$ from the beamline. Nonetheless, several experiments have run in the past which studied scattering angles down to $5^{\circ}$. This is accomplished by using a prebending septum magnet, positioned between the target chamber and the first quadrupole.

\subsection{Detectors}

There were two main detector systems used throughout the experiment. The Standard Hall A detectors are used for particle identification and tracking in each spectrometer [15]. These include vertical drift chambers and scintillators. Each spectrometer also housed a HAPPEx detector similar to the ones used for the original HAPPEx experiment. The two systems can be used together for $Q^{2}$ and background measurements. 

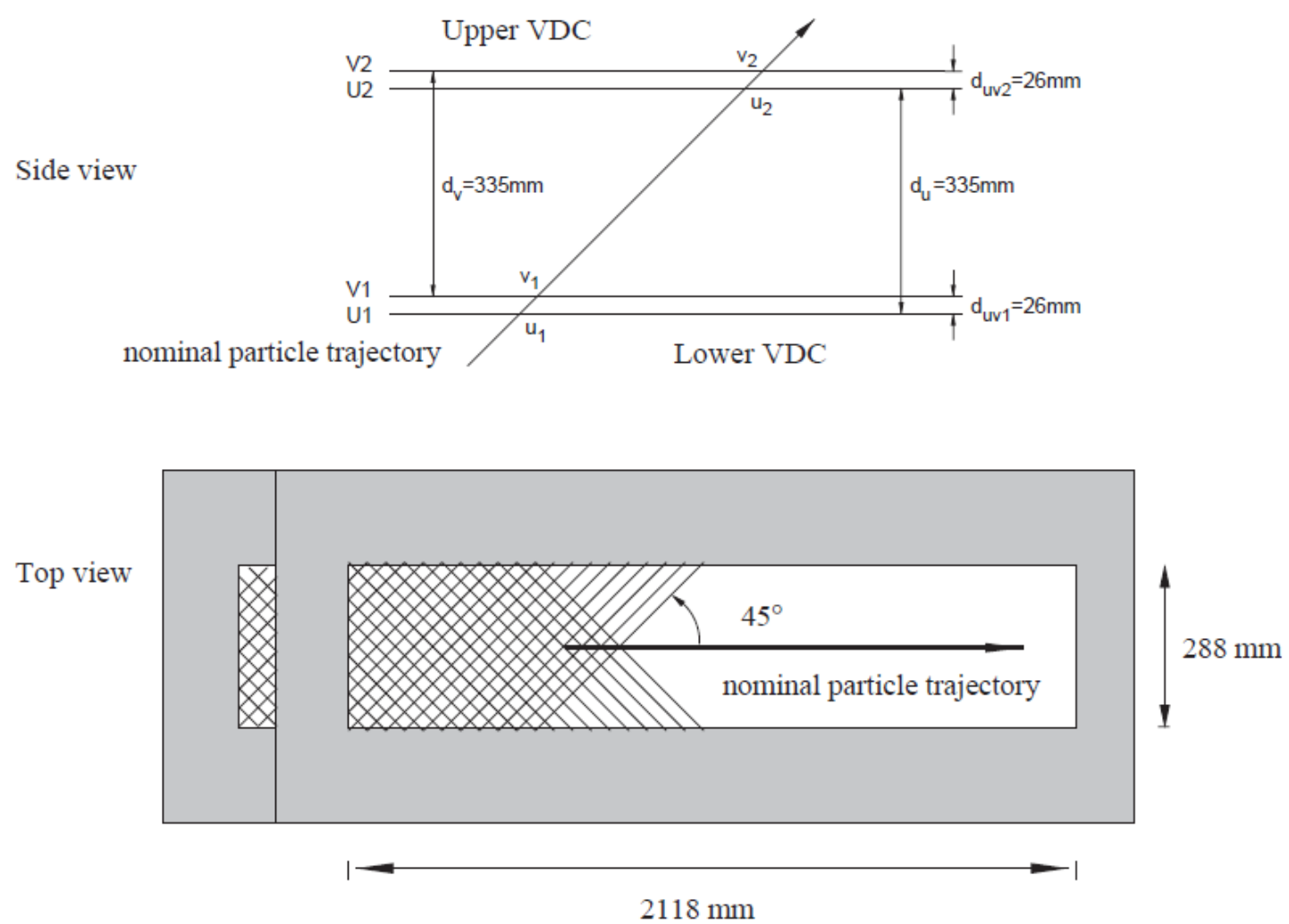

Figure 2.10: Side and top view of the Vertical Drift Chambers used in each Spectrometer.

\subsubsection{Standard Hall A Detector Package}

A pair of vertical drift chambers in each spectrometer provide information about the position and direction of charged particles. Each chamber is made up of two wire planes, with each wire oriented $90^{\circ}$ to one another (see Figure 2.10). They are separated by $335 \mathrm{~mm}$ and lie on a plane that is oriented $45^{\circ}$ from the nominal particle trajectory. When a charged particle goes through them, the electric field inside the VDC is disturbed, creating a signal in the wires that can be read out with the standard DAQ.

Two scintillator planes, S0 and S2, were used as triggers for the data acquisition system. These planes are made up of six overlapping paddles of thin plastic scintillator. The paddles are oriented normal to the nominal particle trajectory and serve to direct light from charged particles that pass through them to two photomultiplier tubes on each side. 
Throughout the experiment, dedicated runs were taken at low beam currents in which these detectors were used to measure background contributions, to determine the average momentum transfer of the detected flux. During regular production data taking, when the beam current and detected rates were high, this equipment was turned off to avoid any damage to the electronics and PMTs.

\subsubsection{HAPPEx Detector}

The design of the HAPPEx detectors, used to collect data in integration mode, is very similar to the one used for the original HAPPEx measurement [44]. These focal-plane detectors are total absorption Cherenkov-shower calorimeters made up by alternating 4 layers of lead and 5 layers of acrylic lucite, materials chosen because of their radiation hardness.

An important specification during the design of the detectors is to optimize their energy resolution. Poor resolution will degrade the integrated signal by increasing the statistical width of the physics asymmetry

$$
\sigma=\frac{1}{\sqrt{N}} \sqrt{1+\left(\frac{\Delta E}{E}\right)^{2}},
$$

where $N$ is the number of scattered electrons detected per integration window, $E$ is the scattered electron energy and $\Delta E$ is the energy resolution. The optimization is accomplished by choosing the proper thicknesses of the layered detector design via GEANT simulations. An energy resolution of $\approx 15 \%$ only broadens the statistical width by about $1 \%$.

When an electron hits the first layer of lead, the resulting shower of electrons emit Cherenkov radiation as they travel through the lucite. The light produced is transmitted with high efficiency toward a 5-inch Burle 8854 photomultiplier tube (PMT). When properly calibrated, the output current of the PMT is proportional to the flux of electrons incident on the detector. Several 'in situ' tests were done to show that the detectors were linear to better than $0.5 \%$ at high currents [23]. The PMT is connected to an analog-to-digital converter (ADC) that integrates the output current. These ADCs are part of the HAPPEx DAQ, made up of several ADCs, scalers and the HAPPEx Timing Board, which controls the rate at which the signals are integrated.

Each ADC channel was calibrated specifically for the expected signal output size 


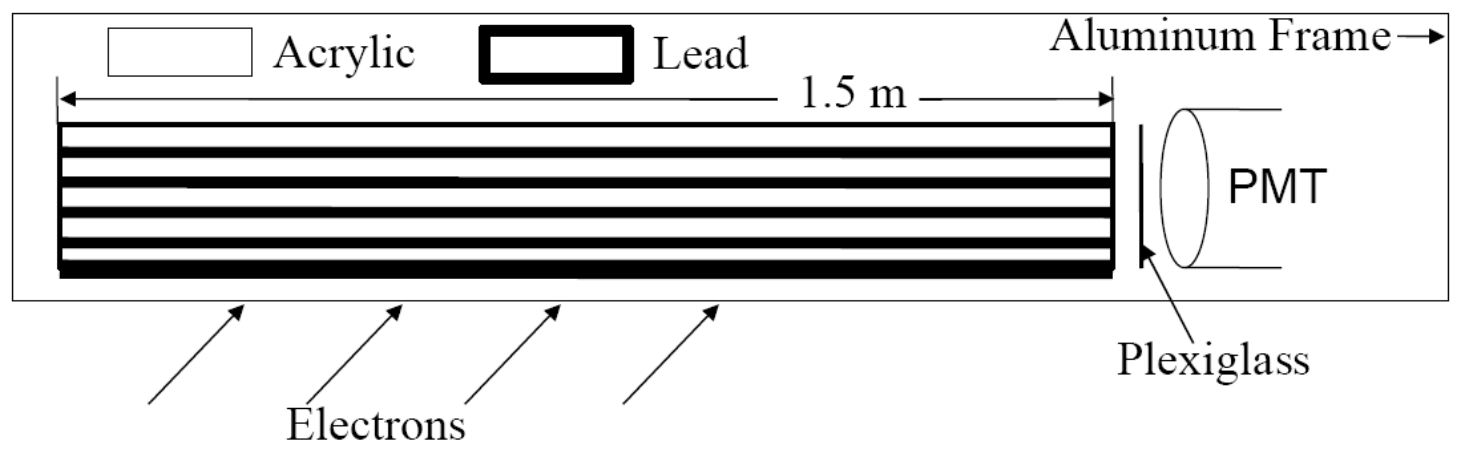

Figure 2.11: The HAPPEx detectors are composed of alternating layers of Lead and Acrylic and a 5-inch PMT housed inside an Aluminum frame.
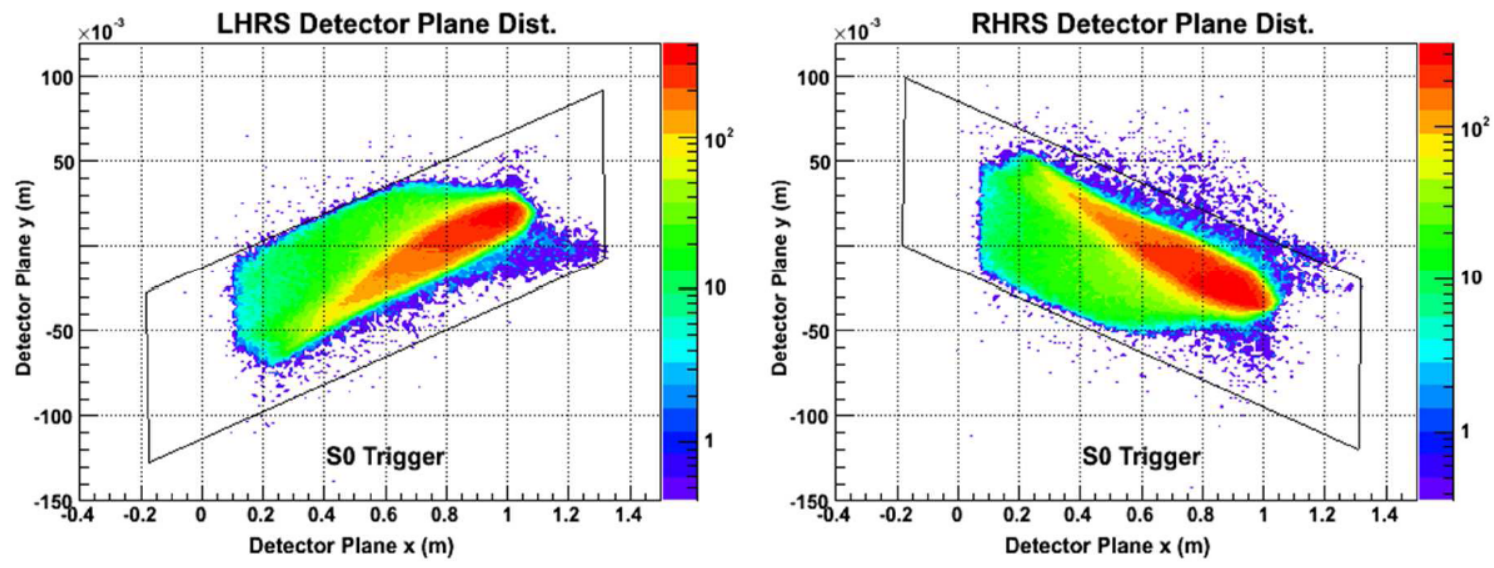

Figure 2.12: Data taken using the standard Hall A detector package and the Counting DAQ. This data shows the alignment of the elastic peak in each Spectrometer. 
of each detector. A 16-bit ADC was used for the detector in the Left HRS and an 18-bit ADC was used in the Right HRS. The full signal of each ADC was about three fourths of their maximum range, designed to be about $5 \mu \mathrm{A}$ at a beam current of 100 $\mu A$. These integrated signals were eventually be used to calculate the asymmetry of elastically scattered electrons. To make sure that the right kind of particle hits the focal plane, the standard VCD and scintillator detectors are used to find the location of the elastic peak. Figure 2.12 shows VDC counts at each detector location, outlined by the actual detector locations on that plane. These plots show that when properly aligned, the detectors are sensitive to the majority of the elastic electrons.

\subsection{Data Acquisition}

Two major data acquisition systems were used throughout data taking. The standard Hall A system, referred to as the 'Counting Mode' DAQ, was used at low currents for detector alignment and measurements of $Q^{2}$. Another system, the HAPPEx 'Integrating Mode' DAQ, was used for our primary detectors, current monitors and other beamline components during the asymmetry measurement.

\subsubsection{Standard DAQ}

The standard Hall A DAQ is used to provide track reconstruction to the target and the detector focal plane event by event [15]. It does so by using the scintillator and HAPPEx detectors to trigger the readout of the Vertical Drift Chambers. When the triggers pass a certain threshold and they coincide, a Trigger Supervisor module signals the DAQ to read out the data, which is then sent to an Event Builder on a Linux workstation. An Event Recorder then writes the data to a disk for later analysis. The entire process is controlled by a software toolkit developed at JLab called CODA (CEBAF On-line Data Acquisition System). This toolkit was designed to facilitate quick and easy changes in the configuration of the DAQ electronics.

\subsubsection{Integrating Mode}

The integration method used in this experiment is ideal for maintaining the high rate necessary to achieve the small statistical error required for this experiment. Instead of 


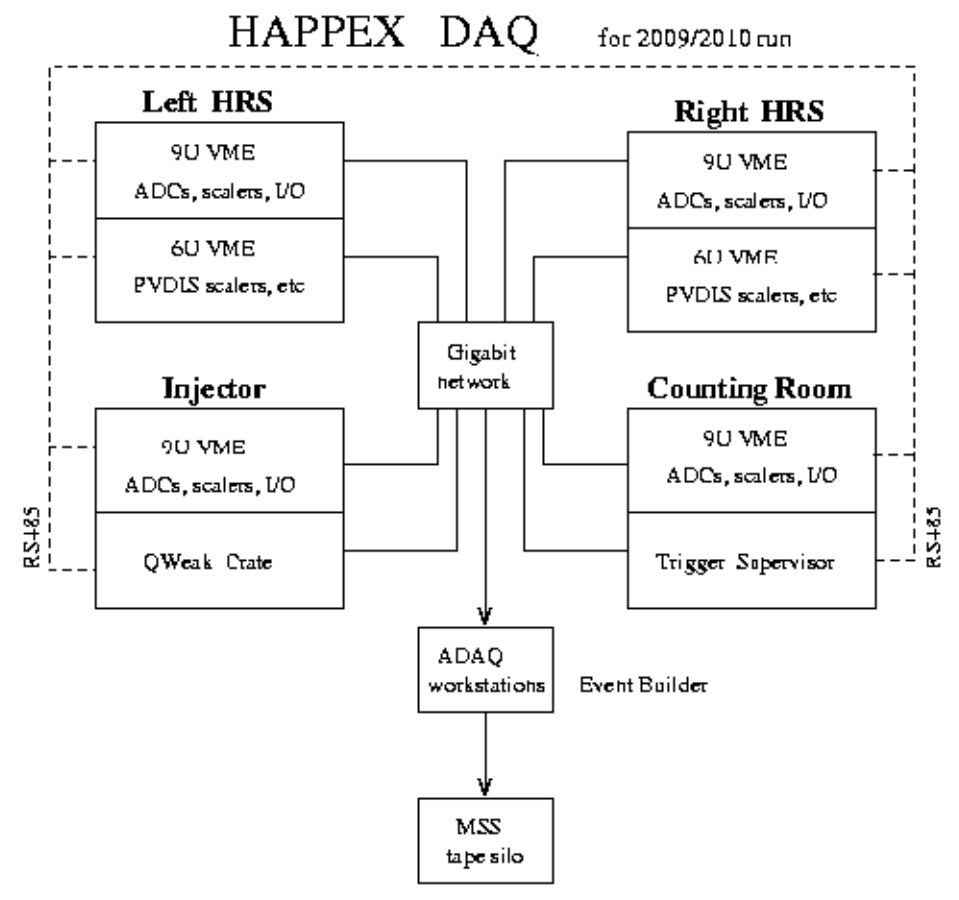

Figure 2.13: Diagram of all HAPPEX DAQ components.

counting individual events, which can lead to DAQ 'dead-time', this method integrates the detected flux over each $33 \mathrm{~ms}$ beam helicity state (or 'helicity window'). The signal flux is measured by the HAPPEx detectors, specially designed total absorption Cherenkov calorimeters which are sensitive to the incident scattered particles. The light produced is detected by a photomultiplier tube and then digitized by 16- and 18-bit ADCs.

The HAPPEx Data Acquisition system is composed of various electronics crates situated all over the accelerator, at the Injector, Counting House and the Left and Right Spectrometer huts in Hall A (see Figure 2.13). Each crate is composed of a Trigger Supervisor, a HAPPEx Timing Board and numerous scalers and ADCs. The Trigger Supervisor is a master signal that drives the start of integration at all the individual crates and keeps them synchronized during data taking. Several tests were conducted throughout the experiment to ensure this synchronization and discussed in Appendix C. The HAPPEx timing boards were designed to control the way signals are integrated, dependent on the structure of the beam helicity signals originating near the polarized source. 


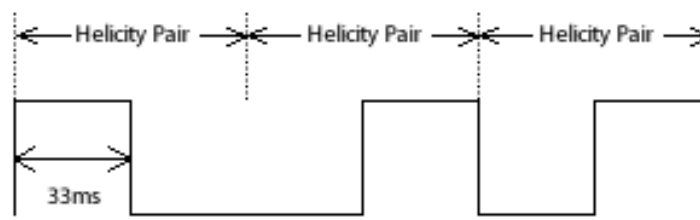

Helicity

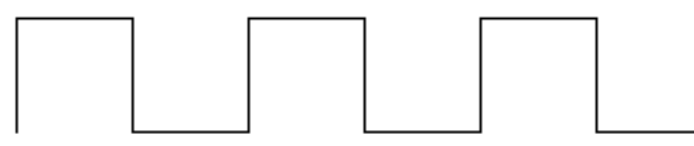

PairSync

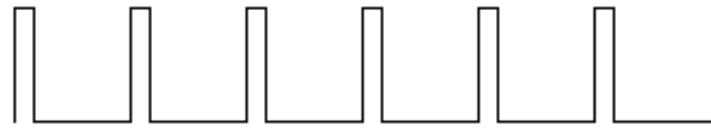

MPS

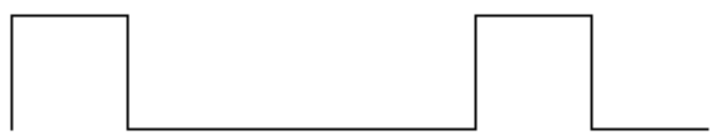

QuadSync

Figure 2.14: Displayed above are all four signals related to the pseudo-random helicity flips of the polarized electron beam. The top signal, labeled 'Helicity' defines the state of the beam polarization. The other signals are used to define the begining of a window, a pair and a quad (two pairs).

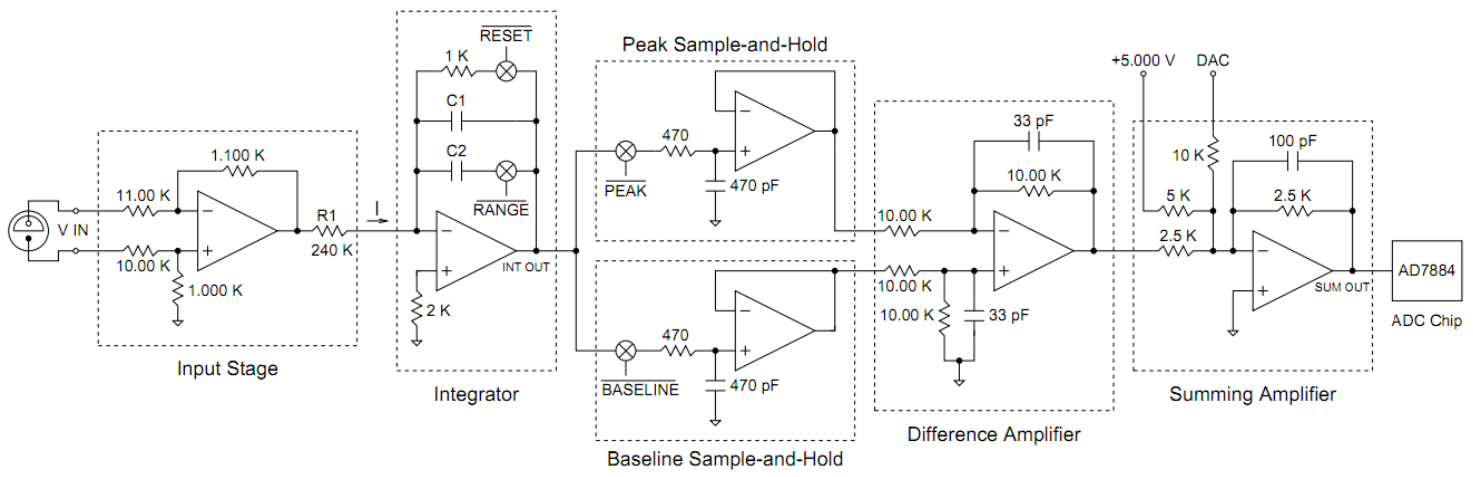

Figure 2.15: Circuit diagram of the integrating circuit for the 16-bit ADCs. 
The main Helicity signal is generated using a number generator that creates a pseudo-random binary sequence at $15 \mathrm{~Hz}$, as shown in Figure 2.14. The second window in each pair is always the complement of the first. This signal corresponds to the actual positive or negative helicity state and is used along with three other signals to properly analyze the data and calculate asymmetries for correct helicity pairs. The PairSync signal is used to distinguish between the first and second window in every pair, characterized as being ON during the first window and OFF during the second. The MPS, or master pulse signal, tells the timing board when a new helicity window starts by being ON for $250 \mu \mathrm{s}$ and $\mathrm{OFF}$ for the rest of the window, at which point the DAQ should start integrating. The width of this pulse is important because it gives the Pockels cell time to settle into its new helicity state. Finally, the QuadSync signal merely groups the windows into sets of four for other analysis purposes.

All the signals of interest, such as beam current monitors (BCMs), beam position monitors (BPMs) and detectors are connected to high-resolution 16-bit and 18-bit analog-to-digital converters (ADCs). Each ADC channel used was tested and modified to accommodate the type of signal being recorded. The 16-bit ADCs were designed for use during the first HAPPEx experiment and their functionality is well known [24]. The 18-bit ADCs were developed for use in the PREx experiment [25]. After extensive testing, they were deployed to be used during HAPPEx since, over time, some of the 16-bit ADCs stopped working. More details on the commissioning of these new high-resolution ADCs will be discussed in Chapter 3.

\subsection{Polarimetry}

The equation used to find the final physics asymmetry shows how the degree of polarization of the electron beam becomes a dilution factor for the detected asymmetry. Even though the source optics are set up to maximize the beam polarization, certain factors make it impossible to achieve $100 \%$ polarization. For this reason, careful determination of the beam polarization is essential for any parity violation experiment. During HAPPEx-III two independent methods used Moller and Compton scattering to make this measurement. The main difference between the two is that the Moller Polarimeter measurement is an invasive procedure while the Compton Polarimeter can run throughout the experiment without affecting the quality of the data. 


\subsubsection{Moller Polarimeter}

The Moller Polarimeter in Hall A uses electron-electron [Moller] scattering to make a measurement of the longitudinal polarization of the beam. This is done by aiming the polarized electron beam toward the atomic electrons in a magnetized ferromagnetic foil. The cross-section of the Moller interaction depends on the polarization of the beam $P^{\text {beam }}$ and the target $P^{\text {target }}$.

$$
\sigma \propto\left[1+\Sigma_{i=X, Y, Z}\left(A_{i i} \cdot P_{i}^{\text {target }} \cdot P_{i}^{\text {beam }}\right)\right]
$$

where $i=X, Y, Z$ are the projections of the polarization and $A_{i i}$ is the analyzing power of Moller scattering. The analyzing power depends on the scattering angle in the center of mass frame

$$
\begin{aligned}
A_{Z Z} & =-\frac{\sin ^{2} \theta_{C M}\left(7+\cos ^{2} \theta_{C M}\right)}{\left(3+\cos ^{2} \theta_{C M}\right)^{2}}, \\
A_{X X} & =\frac{\sin ^{4} \theta_{C M}}{\left(3+\cos ^{2} \theta_{C M}\right)^{2}}, \\
A_{Y Y} & =-A_{X X}
\end{aligned}
$$

where it is assumed that the beam direction is along the Z-axis and that the scattering is in the ZX plane.

The Moller Polarimetry measurement is done by aiming the electron beam at a target foil at symmetric angles $\pm 20^{\circ}$ with respect to the beam direction, where any transverse components of the beam polarization have opposite signs and will cancel out when averaging them. This method also reduces the impact of uncertainties in the target angle measurement [15].

\subsubsection{Compton Polarimeter}

Another method to measure the polarization of the electron beam uses Compton scattering $\left(e^{-} \gamma \rightarrow e^{-} \gamma\right)$. The Compton Polarimeter studies the interaction between longitudinally polarized electrons and circularly polarized photons in a Fabry-Perot cavity $[33,34]$. The polarization of the electron beam can be described as

$$
P_{e}=\frac{N_{e}^{+}-N_{e}^{-}}{N_{e}^{+}+N_{e}^{-}}
$$

where $N_{e}^{+(-)}$is the number of electrons with spin parallel (antiparallel) to the beam direction. To find the value of $P_{e}$, we measure the asymmetry between the integrated 

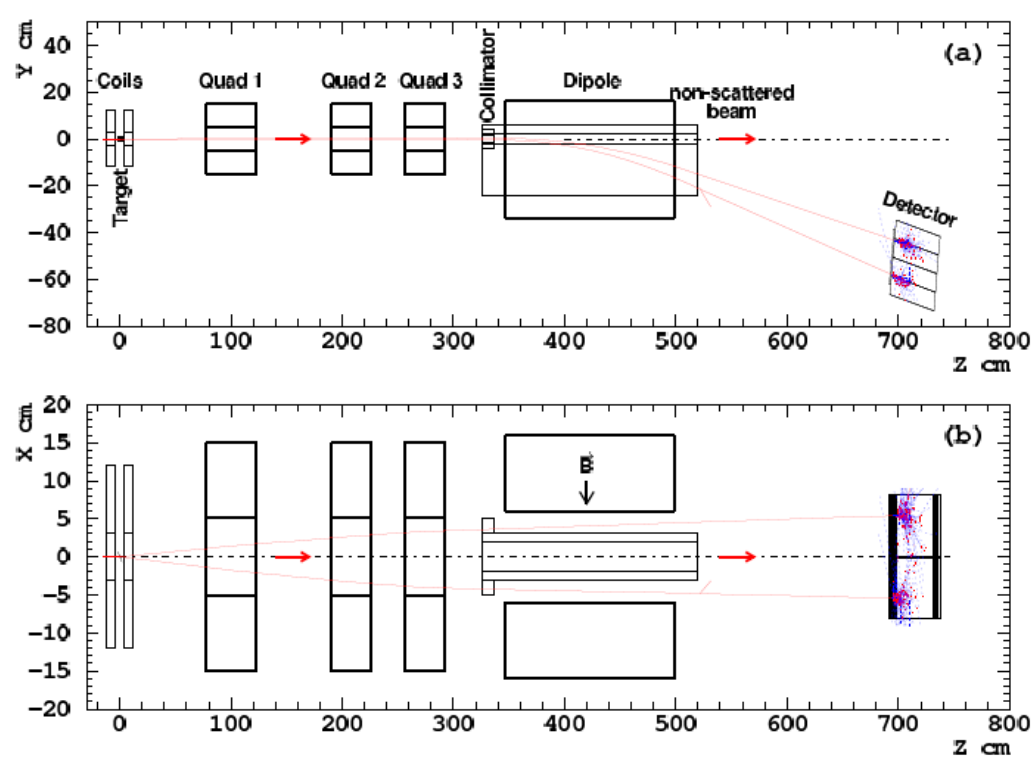

Figure 2.16: (a) Side view and (b) top view of the Moller Polarimeter. Each plot shows simulated events that hit the detectors.

Compton signal $S$ at different helicity states

$$
A_{\text {meas }}=\frac{S^{+}-S^{-}}{S^{+}-S^{-}}=P_{e} P_{\gamma}\left\langle A_{S}\right\rangle
$$

Since the theoretical asymmetry for Compton scattering $A_{S}$ is known and the photon polarization $P_{\gamma}$ is a measured value, we can find the electron beam polarization using Equation 2.16. The layout of the equipment used to make this measurement is shown in Figure 2.17. The Compton Polarimeter is located in the area right before the beam enters Hall A. Figure 2.18 shows a more detailed schematic of this setup

The Compton Polarimeter is designed so that is has a minimal effect on the electron beam (only one electron in $10^{9}$ undergoes Compton scattering) and can run throughout the course of the experiment. The polarimeter is composed of a chicane formed by four dipoles, a Fabry-Perot cavity, an electron detector and a photon detector. After entering from the left (see Figure 2.18), electrons interact with a circularly polarized green light $(\lambda=532)$ laser beam kept in resonance inside the cavity, located between the second and third dipoles. While the primary beam goes through the chicane and into the experimental hall, the scattered electrons are deflected toward an electron detector made up of a silicon microstrip and the scattered photons are detected with a GSO crystal calorimeter. 


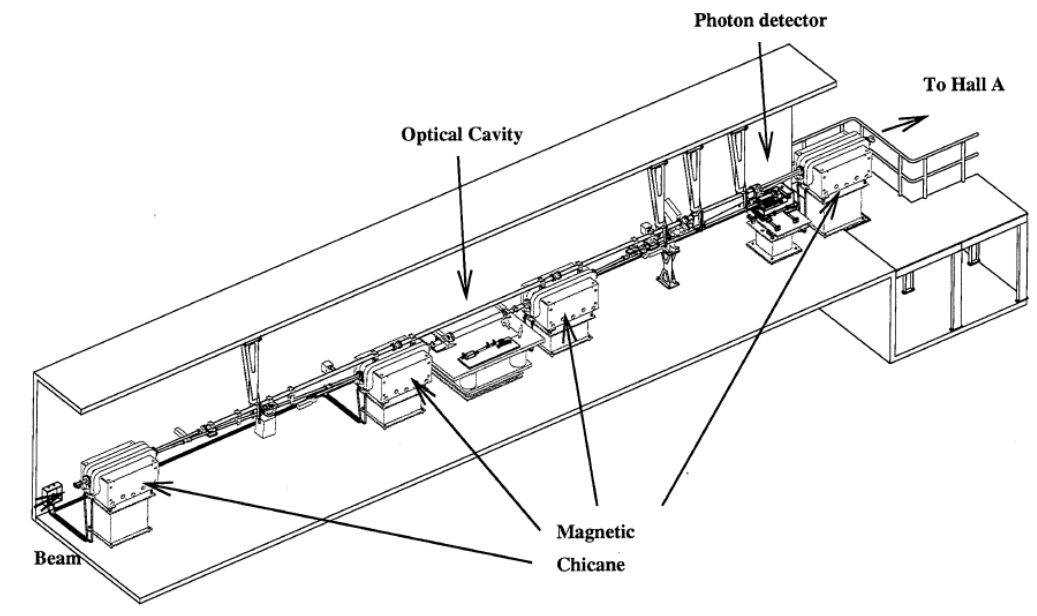

Figure 2.17: The Compton Polarimeter is located where the beam enters Hall A.

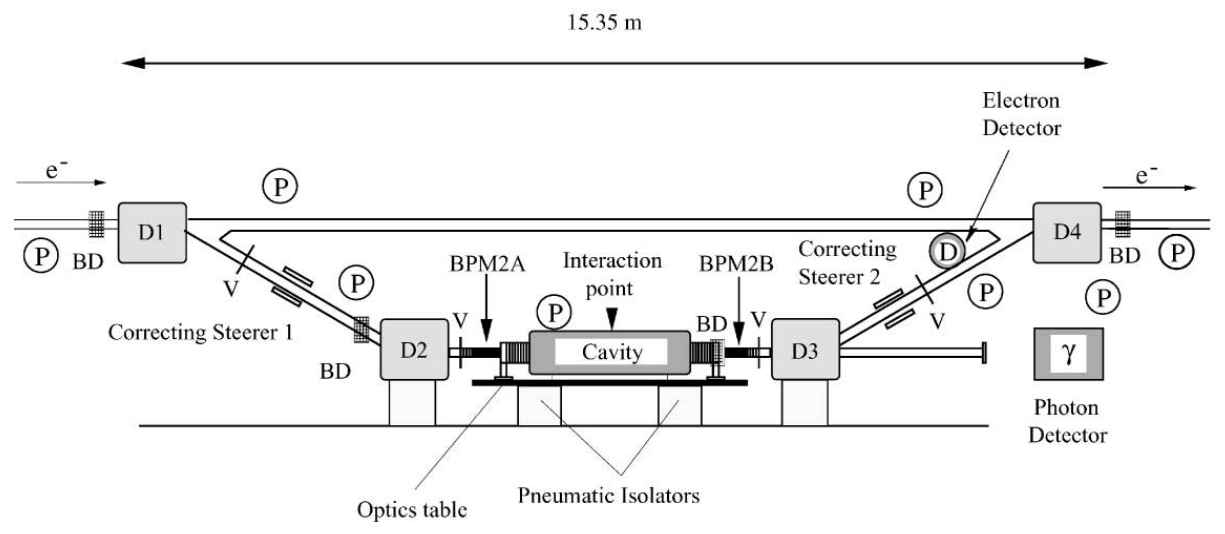

Figure 2.18: A detailed schematic of the Compton Polarimeter components. 


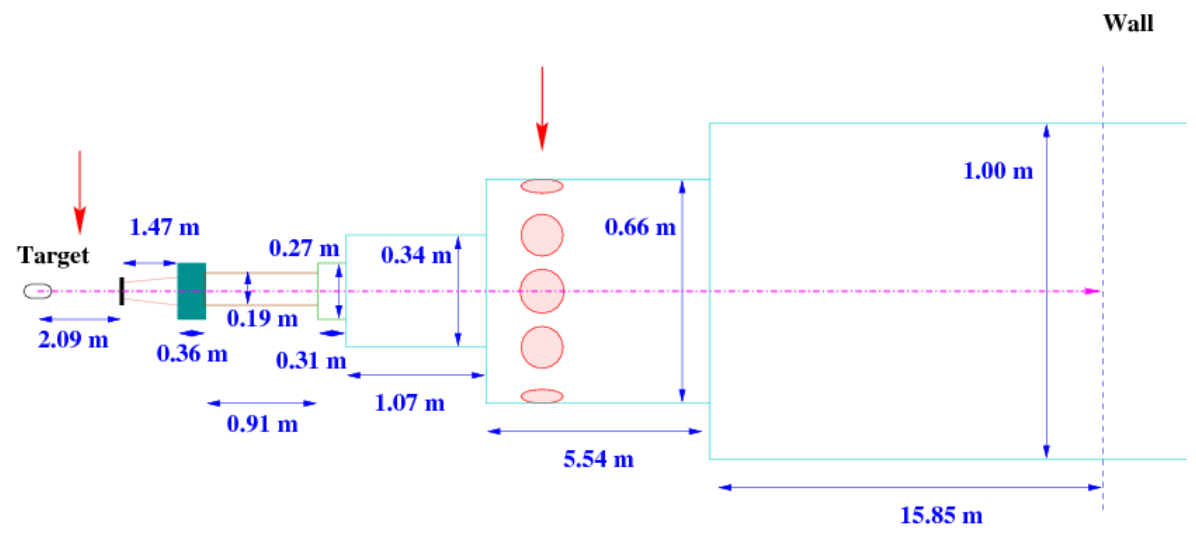

Figure 2.19: Schematic of beam pipe showing location of luminosity monitors relative to the target

For the HAPPEx-III experiment, the precision of the polarization measurement was required to be $1 \%$ or better. The Fabry-Perot cavity helped achieve this by providing a high photon flux when kept in resonance [26]. It is also important to optimize the crossing angle between the photon and electron beams inside the cavity to maximize the luminosity of the scattered signals. To reduce helicity-correlated systematic errors, the laser state varies every few minutes between left- and rightcircular polarization.

\subsection{Luminosity Monitor}

The luminosity monitors, 'lumis' for short, consist of eight Cherenkov detectors symmetrically placed around the primary beam. They are located $7 \mathrm{~m}$ downstream of the target, as shown in Figure 2.19. Each detector is made up of a small rectangular piece of fused quartz, a long aluminum air light guide, a filter box and an R7723 PMT (see Figure 2.20). Every PMT used as a luminosity monitor was studied throughly to learn about its gain and good linearity settings.

The light guide is long enough so that the luminosity monitor is sensitive to very small scattering angles $\left(0.5^{\circ}\right)$, roughly where the quartz piece is located. When the scattered particles hit the quartz, Cherenkov light is produced and travels up the aluminum air light guides. The light reaches the PMT photocathode and produces an electrical signal that can be measured with our electronics. The filter box is 


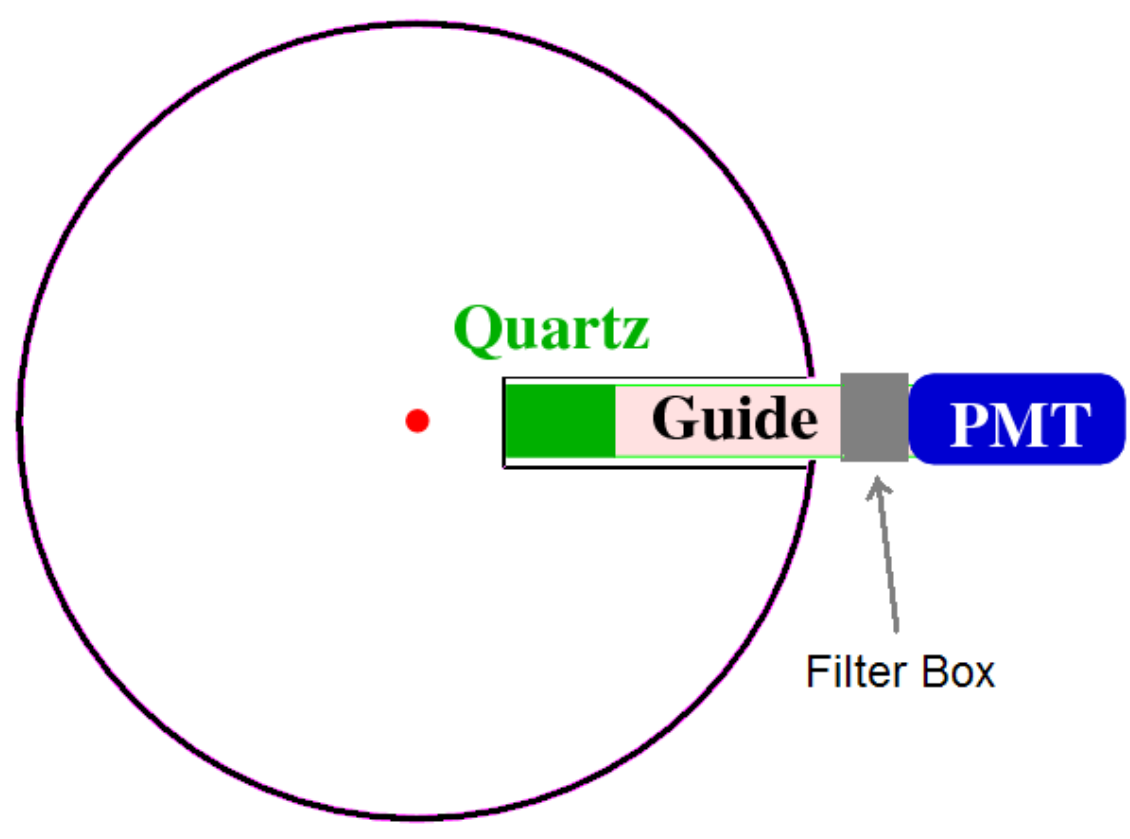

Figure 2.20: Simple schematic of a singleLuminosity Monitor

used when very high rates are expected in order to avoid any non-linearities related to saturation of the PMT. At these small scattering angles, any helicity-correlated asymmetries should go to zero because $Q^{2}$ is close to zero.

The PMT signal is integrated using the HAPPEx DAQ and the width of its asymmetry can be used as a lower limit on the electronics noise. Because the rates hitting the fused quartz are high at such low angles, the integrated signal will have a small statistical width and can be used to quickly detect helicity-correlated beam fluctuations. They can also be useful to monitor any fluctuations in the density of the targets themselves. During HAPPEx data taking, several studies were conducted to determine the optimal conditions for minimum target boiling, the results of which are presented in Appendix D. 


\section{CHAPTER 3}

\section{PREX PREPARATIONS}

Another parity-violation experiment proposed in 1999 aimed at measuring the weak charge distribution of a heavy nucleus [10]. As was discussed in Section 1.7, the ultimate goal of the experiment was to measure the neutron RMS radius of the lead nucleus to unprecedented accuracy. To achieve a $1 \%$ measurement in the RMS radius, a $3 \%$ measurement of the sub-ppm parity-violating asymmetry must be accomplished. Making such a precise measurement required stringent controls over sources of noise and false asymmetries.

Several new components, such as 18-bit ADCs, a new Septum magnet and a specially designed integrating detector package, were tested and installed in Hall A to accomplish this measurement. Several upgrades to the source optics, including a Double Wien Filter configuration, served to keep helicity-correlated beam properties under control. These and other matters, such as target design and stability, will be discussed in detail in this chapter.

The Lead Radius Experiment (PREx) had a successful physics run in the Spring of 2010 with a set of experimental conditions designed to minimize the running time needed to achieve the $3 \%$ accuracy required in the asymmetry measurement. Table 3.1 summarizes the proposed kinematic conditions. Some problems were encountered during production mode running which did not allow the collaboration to collect enough data to achieve the statistical error goal. Nonetheless, the systematic error goal of $2 \%$ was successfully achieved due to the various new techniques used throughout the experiment. 


\begin{tabular}{lr}
\hline Measured Asymmetry & $0.51 \mathrm{ppm}$ \\
\hline Beam Energy & $1.05 \mathrm{GeV}$ \\
\hline Beam Current & $50 \mu \mathrm{A}$ \\
\hline Beam Polarization & $80 \%$ \\
\hline Target & $10 \%$ r.l. $\mathrm{Pb}$ \\
\hline Scattering Angle & $5^{\circ}$ \\
\hline Required Statistical Accuracy & $3 \%$ \\
\hline Energy Cut (due to detector) & $4 \mathrm{MeV}$ \\
\hline Detected Rate (each Spectrometer) & $860 \mathrm{MHz}$ \\
\hline Running Time & $30 \mathrm{days}$ \\
\hline
\end{tabular}

Table 3.1: Summary of proposed experimental conditions and kinematics.

\subsection{Overview}

The experimental technique used for PREx was very similar to that used for the HAPPEx-III measurement, described in Chapter 2. The same kind of polarized electron beam was used, this time with a helicity reversal rate of $120 \mathrm{~Hz}$, as opposed to the $30 \mathrm{~Hz}$ rate for HAPPEx-III. Also, a new double wein technique was deployed for slow helicity reversals similar to the slow IHWP flips, used to cancel out helicity correlated systematic effects.

The rest of the changes took place in some of the instrumentation inside Hall A. The main targets for PREx were composed of a thin lead foil sandwiched between two diamond foils. After interacting with the target, elastically scattered electrons are guided by a new room-temperature septum toward specially designed collimators at the entrance of the first quadrupole of each high resolution spectrometer (see Section 2.6).

Each HRS guided the elastic electrons toward a detector package composed of two main quartz Cherenkov detectors and a special detector to monitor transverse asymmetries. New 18-bit ADCs were used in the PREx DAQ to measure BCM, BPM and PREx detector signals. Also, upgrades to both polarimeters made it possible to measure the degree of polarization to better accuracy than ever before. This chapter will go through some of the essential changes between the HAPPEx-III and the PREx experimental setup. 


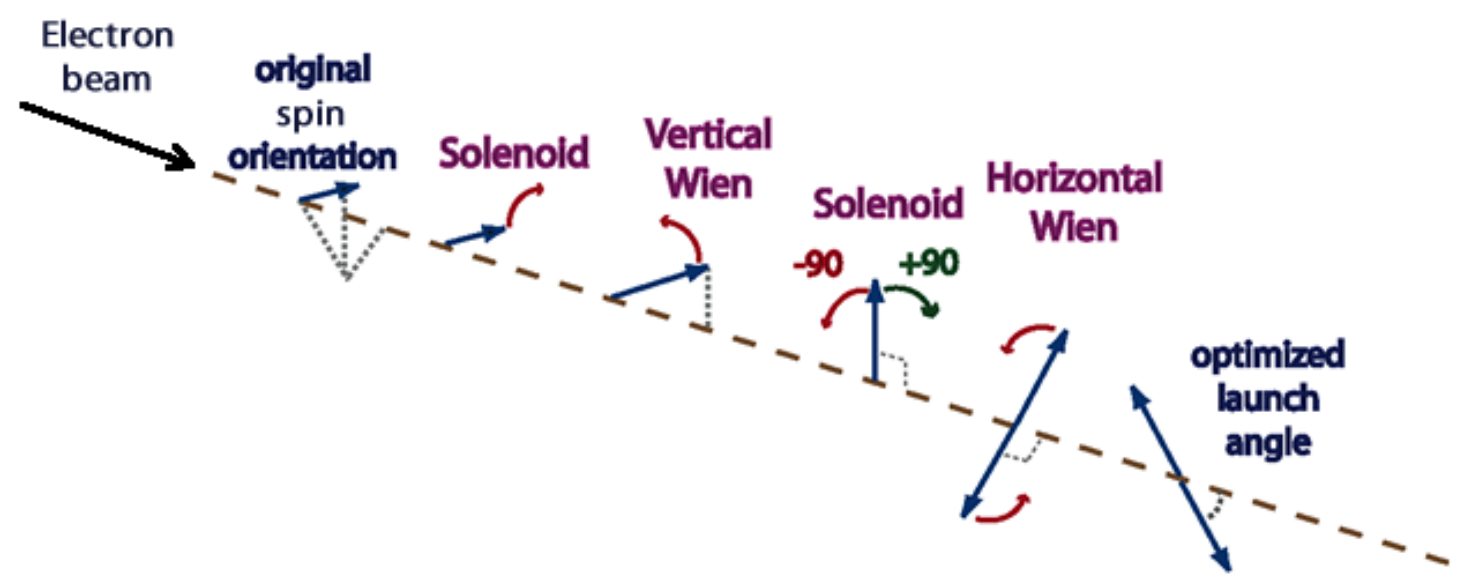

Figure 3.1: Diagram showing all the optical elements used as part of the Double Wien filter setup at the Injector.

\subsection{Double Wien Filter}

The PREx measurement required the strictest controls ever on helicity-correlated false asymmetries. For this reason, an extra set of electromagnetic elements were added to the beamline at the source that would provide a second source of slow helicity reversal. Before this addition, there existed a single Vertical Wien filter which served to fix the launch angle of the electron beam so that its polarization was optimized when it reached the experimental hall. This was necessary to account for the effects of spin precession.

A Wien filter is an optical element with a crossed electric and magnetic field. By balancing the force contributions from both fields, it can be used to change the direction of the beam's polarization without affecting its direction of motion. The longitudinal polarization of the beam can precess due to magnetic fields when travelling down the beam pipe toward the experimental hall. The Wien filter can be used to give the beam a small component of transverse polarization that will cancel out the effects of spin precession [27].

The two new elements, a solenoid and a Horizontal Wien filter (see Figure 3.1), make it possible to passively flip the helicity of the beam without introducing any first-order contributions to false asymmetries. This is done by flipping the solenoid to $\pm 90^{\circ}$. Since the helicity of the beam depends on the sign of the magnetic field inside 
the solenoid, the spin rotation will change signs accordingly. Beam focusing depends on the square of the magnetic field, so that it is unaffected when flipping the spin this way. This provides a suppression of 'second-order' effects such as a helicity-correlated beam spot size. This technique was used as an extra cancellation of small systematic effects that had been unaccounted for in the past.

\subsection{Lead Target}

Designing the target was one of the biggest technical challenges of the experiment. The thermal properties of the lead target had to be improved so that it could withstand the expected beam power without reaching its melting point. The nominal design consisted of a $0.5 \mathrm{~mm}$ foil of ${ }^{208} \mathrm{~Pb}$ sandwiched between two $0.15 \mathrm{~mm}$ sheets of diamond, which is pure ${ }^{12} \mathrm{C}$ (see Figure 3.2). More detailed measurements of the foil thicknesses were conducted and are documented in [28]. The diamond is used to increase target stability due to its high thermal conductivity. These three layers are clamped in a copper block assembly which is cooled by liquid helium (see Figure 3.3).

In January of 2008 the target design was successfully tested up to $100 \mu \mathrm{A}$, twice the beam current proposed for the experiment (see Table 3.1). This high current was incident on the target for about 2 hours with no apparent damage. Still, other tests conducted in the past have shown that these lead targets have a lifetime of about a week when running at such high beam currents.

When putting together the layers of lead and diamond, there must be good thermal contact between them. This maximizes the positive effects of the diamond backing. To accomplish this, a very thin layer of 'vacuum grease' was applied throughout the lead/diamond interface. The three foils are then clamped together and a silver-based paste is applied throughout the area were the diamond and the copper make contact.

\subsubsection{Target Stability}

During PREx running, a few problems were encountered related to the uniformity of the target thickness. Figure 3.4 shows the history of detected rates for all three targets used throughout the experiment. Degradation in the uniformity of the target thickness led to a drop in the detected rates, which resulted in higher pulse-pair 


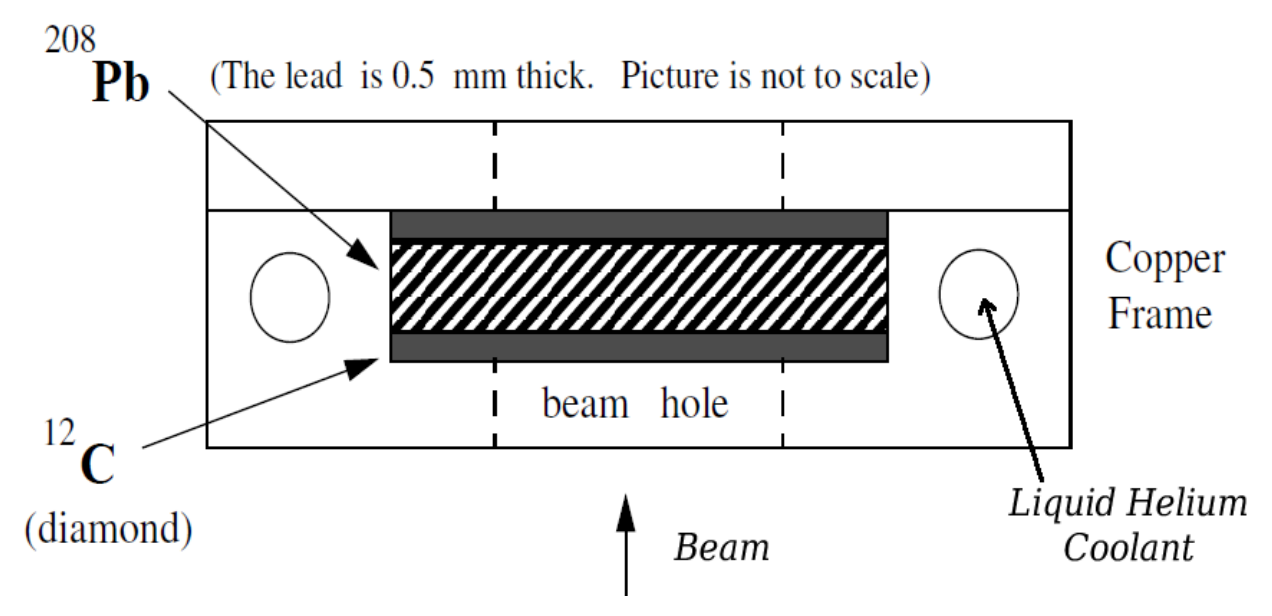

Figure 3.2: Schematic of a cross-section of the PREx target ladder.

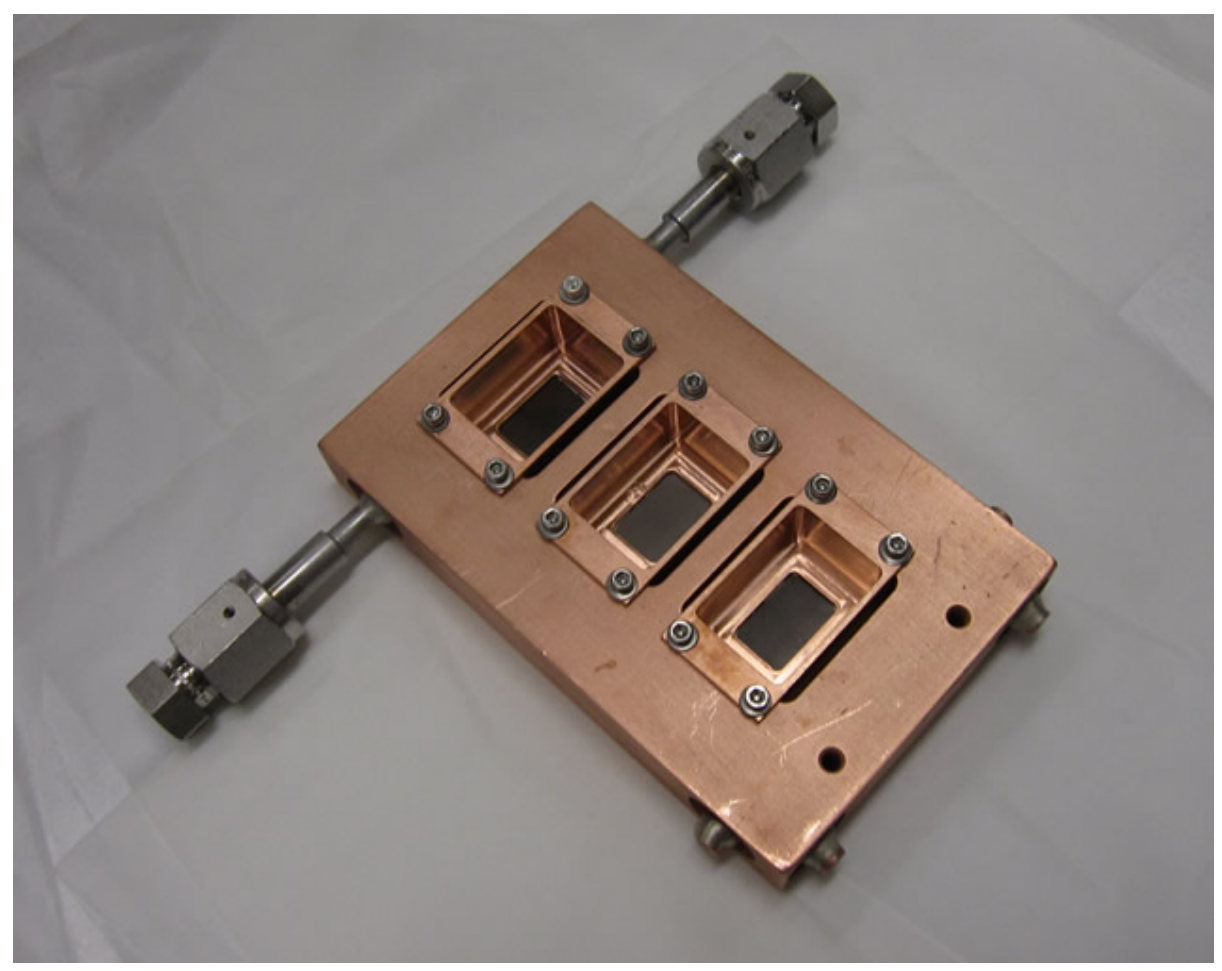

Figure 3.3: Picture of Copper frame used to house the PREx targets. Note the Liquid Helium intake at the top of the frame. 

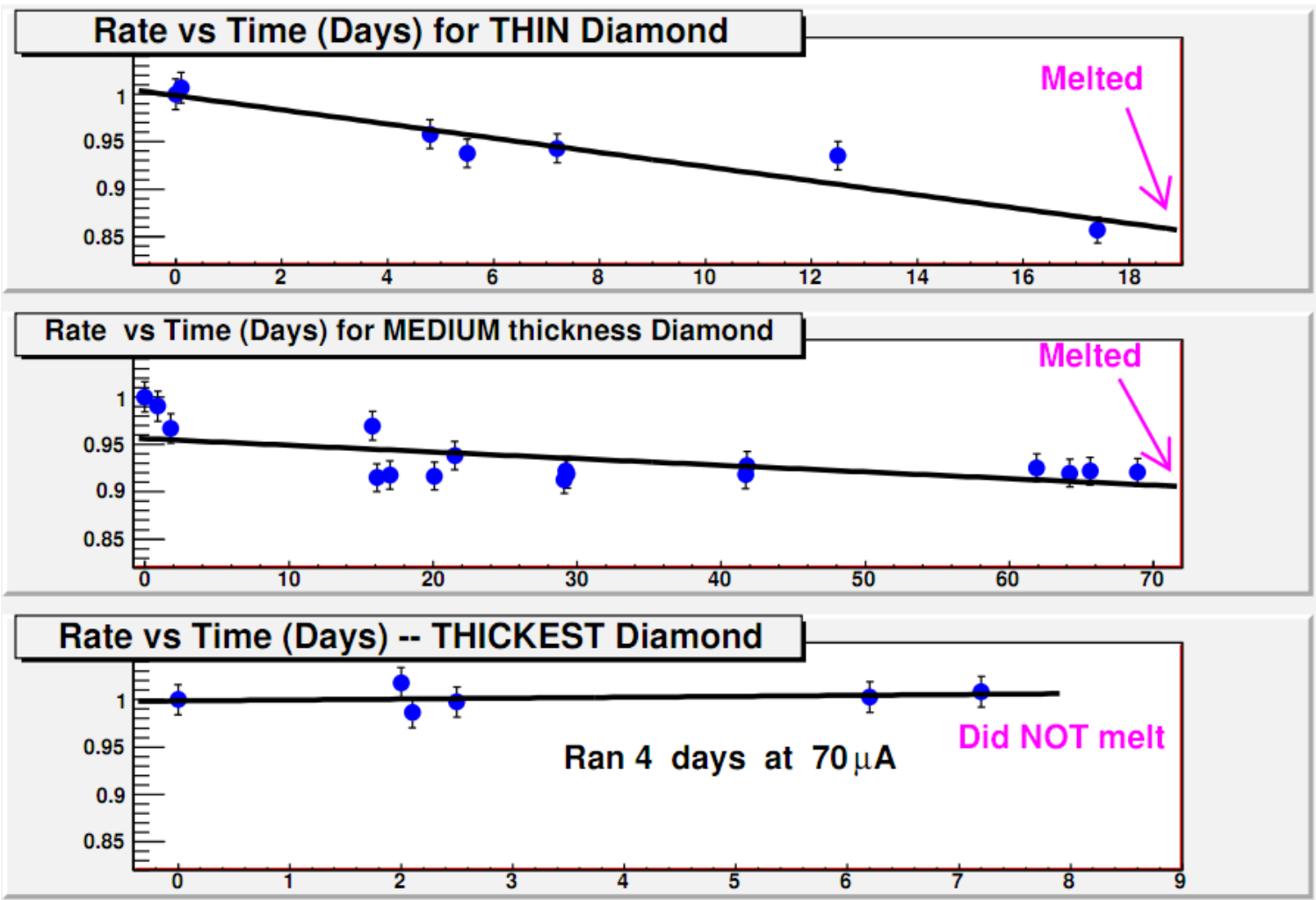

Figure 3.4: Time-dependant target rate measured using counting mode DAQ for the three different Lead-Diamond targets. The target with the thinnest diamond backing (4.5\% background) degraded the fastest. Two of the targets melted. The target with the thickest diamond (8\%) did not melt and ran for 4 days at $70 \mu \mathrm{A}$ (and 7.5 days total) 


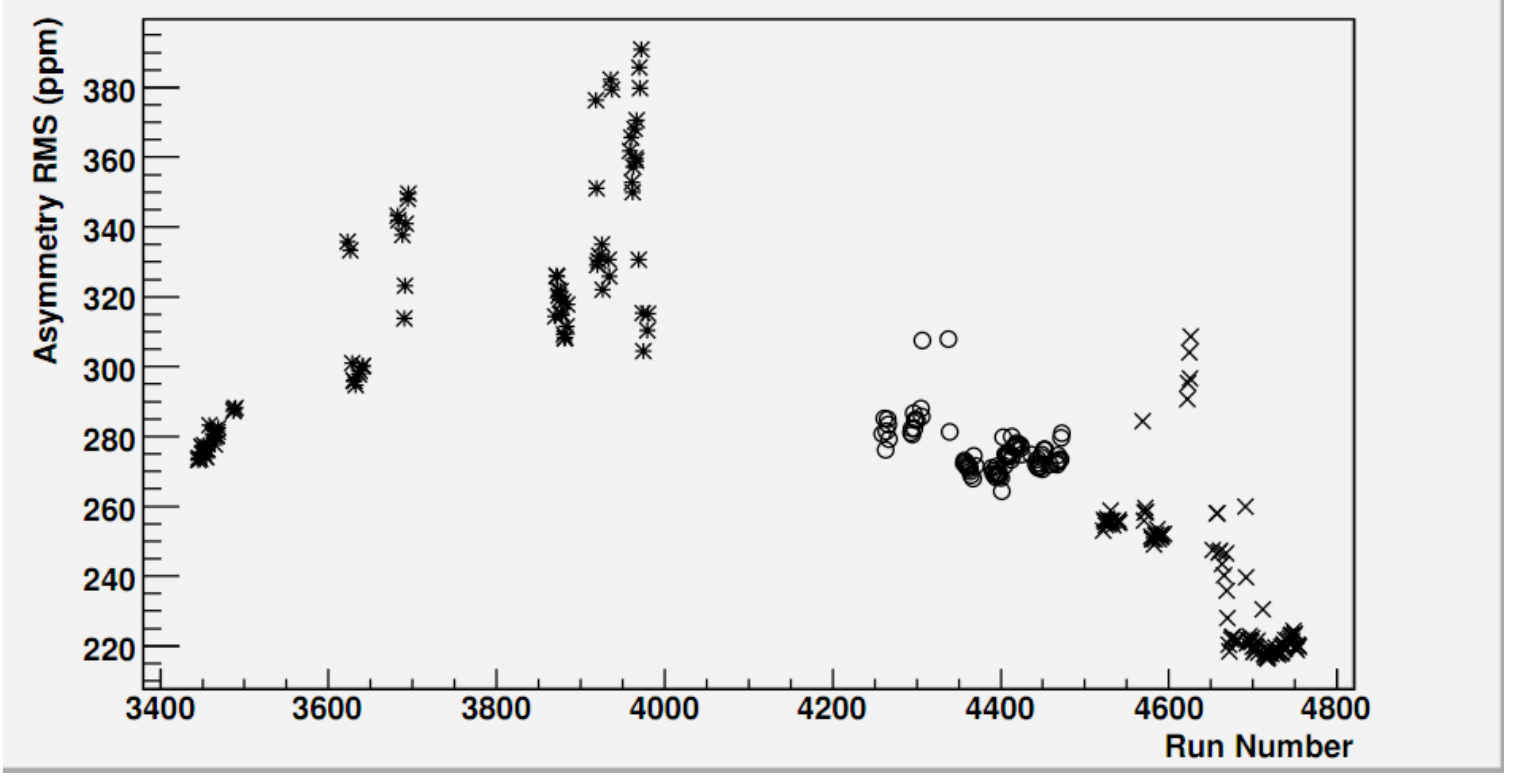

Figure 3.5: Variations in the RMS of the measured detector asymmetry throughout all the data runs. The sudden jump to RMS higher than $300 \mathrm{ppm}$ is a clear indicator of the degradation of the target. While most of the data was taken at $50 \mu \mathrm{A}$, runs higher than 4660 were conducted at $70 \mu \mathrm{A}$.

widths, as seen in Figure 3.5. The essential difference between the three targets was a small variation in the thickness of the diamond foils. The first target used had the thinnest diamond backing and degraded very quickly (about a week's worth of data taking) with a beam current of $50 \mu \mathrm{A}$. The last target used, was fairly stable throughout and was able to withstand a $70 \mu \mathrm{A}$ beam for 4 days.

Figure 3.6 shows the raster profile scan for one of the targets after a hole seemed to develop. This caused extra noise of $\approx 40 \%$ that could be seen as a correlation between the signals measured by the detectors in each spectrometer (see Figure 3.7). Since the two HRS detect different electrons, such correlations should not exist. To address this problem, the raster electronics were modified to develop a precision lock that forced the raster to execute the same orbit between two adjacent helicity cycles. The implemented changes completely cancelled out the extra noise caused by the target degradation when calculating the pair-wise asymmetries. 


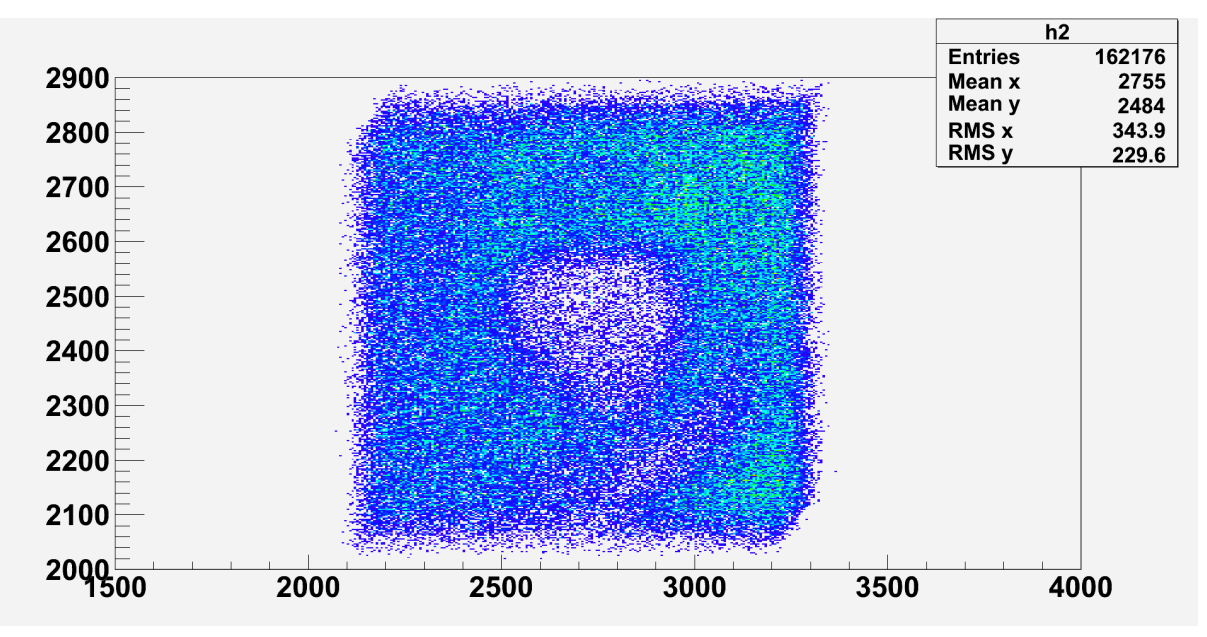

Figure 3.6: Raster scan conducted with the Hall A standard DAQ package shows lower rates at the center of the rectangular raster pattern, indicating degradation of the target and the first steps of melting.
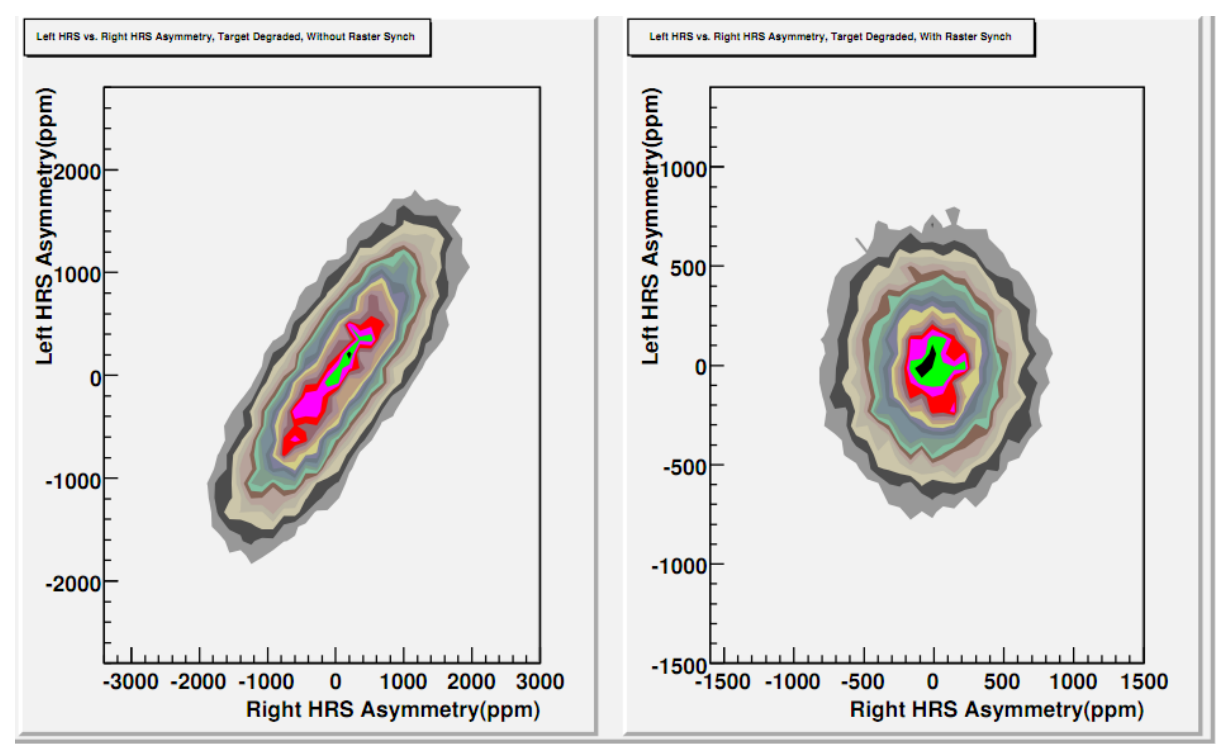

Figure 3.7: The left plot shows the correlation in the pulse-pair asymmetry between detectors in the left and right HRS after target degradation. The right plot shows the same signals after synching the raster with the helicity flip rate. These show that this technique effectively cancelled out the effects of target density fluctuations. 


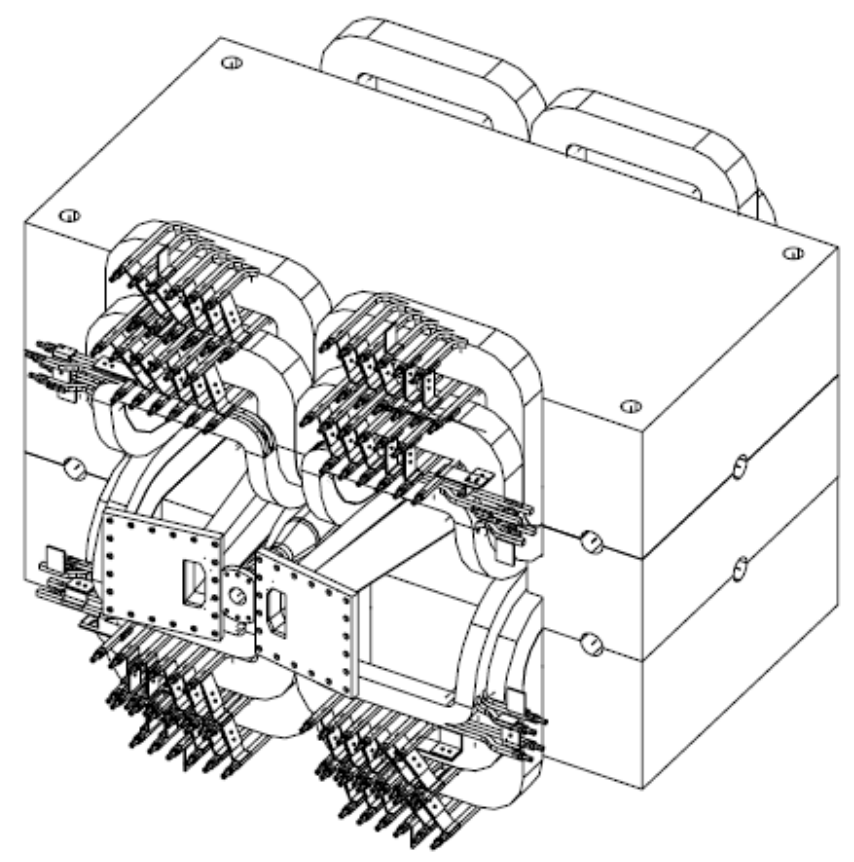

Figure 3.8: Graphical wire frame designs of the new Septum magnet.

\subsection{New Septum}

The High Resolution Spectrometers located in Hall A can only be moved to a minimum scattering angle of $12.5^{\circ}$ from the beam line. For this reason, experiments that are designed with a smaller scattering angle must use a septum magnet between the target and the spectrometers.

The septum magnet is designed to bend the trajectory of the scattered particles toward the spectrometers. Even though experiments such as HAPPEx-II have used superconducting septum magnets in the past that are available for use, they will not work for PREx because the high luminosity expected would induce radiational heating of the superconducting coils. The $5^{\circ}$ Septum magnet required for the PREx experiment is a new room-temperature magnet and has half the magnetic field of the existing superconducting septa. This is possible because the beam energy of the experiment is much lower than previous experiments.

It is challenging to calibrate the magnetic field to get the proper value of $\int B d l$ that optimizes the figure-of-merit (FOM) of the experiment. The FOM for this measurement is the expected error in the value of the neutron RMS radius and is minimized 


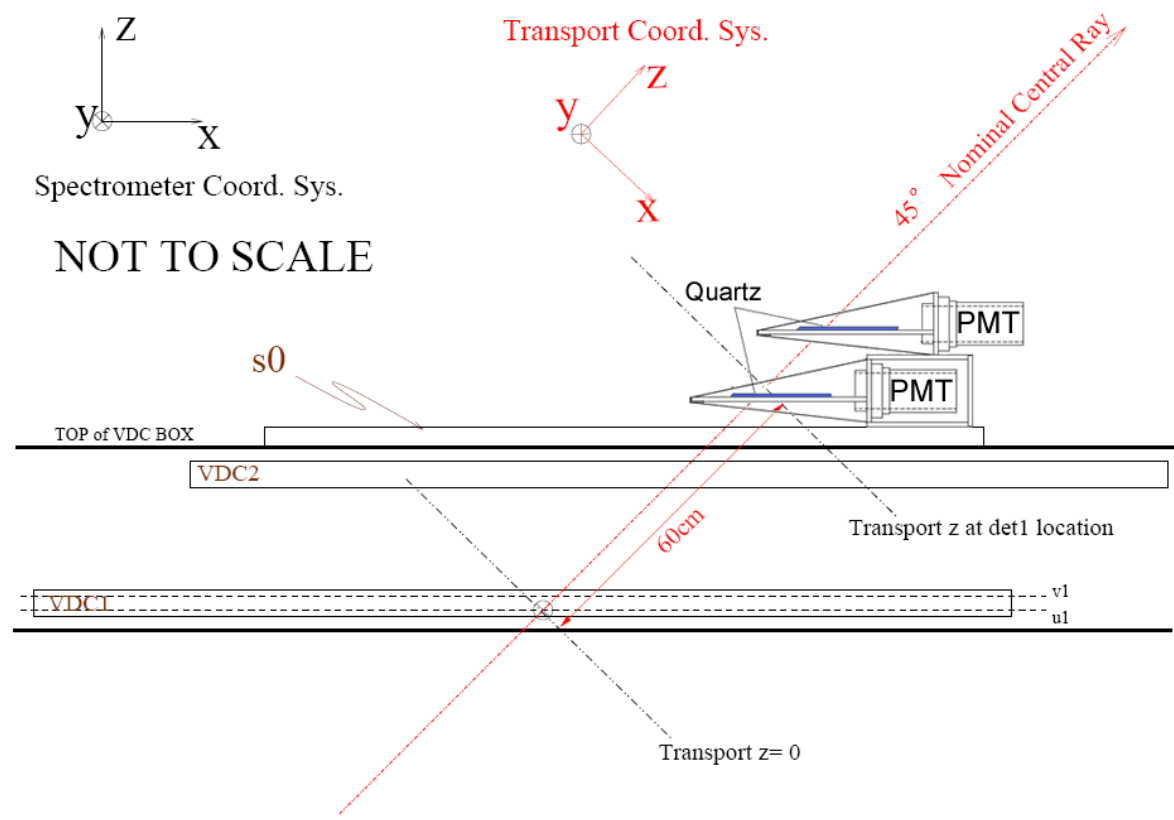

Figure 3.9: Location of the focal plane detectors with respect to the vertical drift chambers and the S0 scintillator.

by maximizing the product $R \times A^{2} \times \epsilon^{2}$, where $R$ is the rate, $A$ is the asymmetry and $\epsilon=d A / A$ is the sensitivity of $A$ to $R_{N}$. After analysis of data taken throughout the experiment, it was found that the magnet strength was set about $6 \%$ lower than the optimal value, leading to lower rates and reducing the FOM by $16 \%$.

\subsection{PREx Detector Package}

A new detector concept was designed by the University of Massachusetts and Smith College for the PREx measurement. The final design was developed after studying the results of a beam test conducted in early 2008 [29]. It consisted of three separate quartz Cherenkov detectors in each spectrometer arm. Two of these detectors, referred to as 'thin' and 'thick' quartz, were located in the focal plane, where they could measure the peak of the elastically scattered electrons. The third one, called the ' $A_{T}$ detector,' was specially positioned to measure a transverse asymmetry arising from a residual vertical polarization. Figure 3.9 shows the design and placement of the focal plane detectors. Each detector is composed of a rectangular slab of quartz housed in 


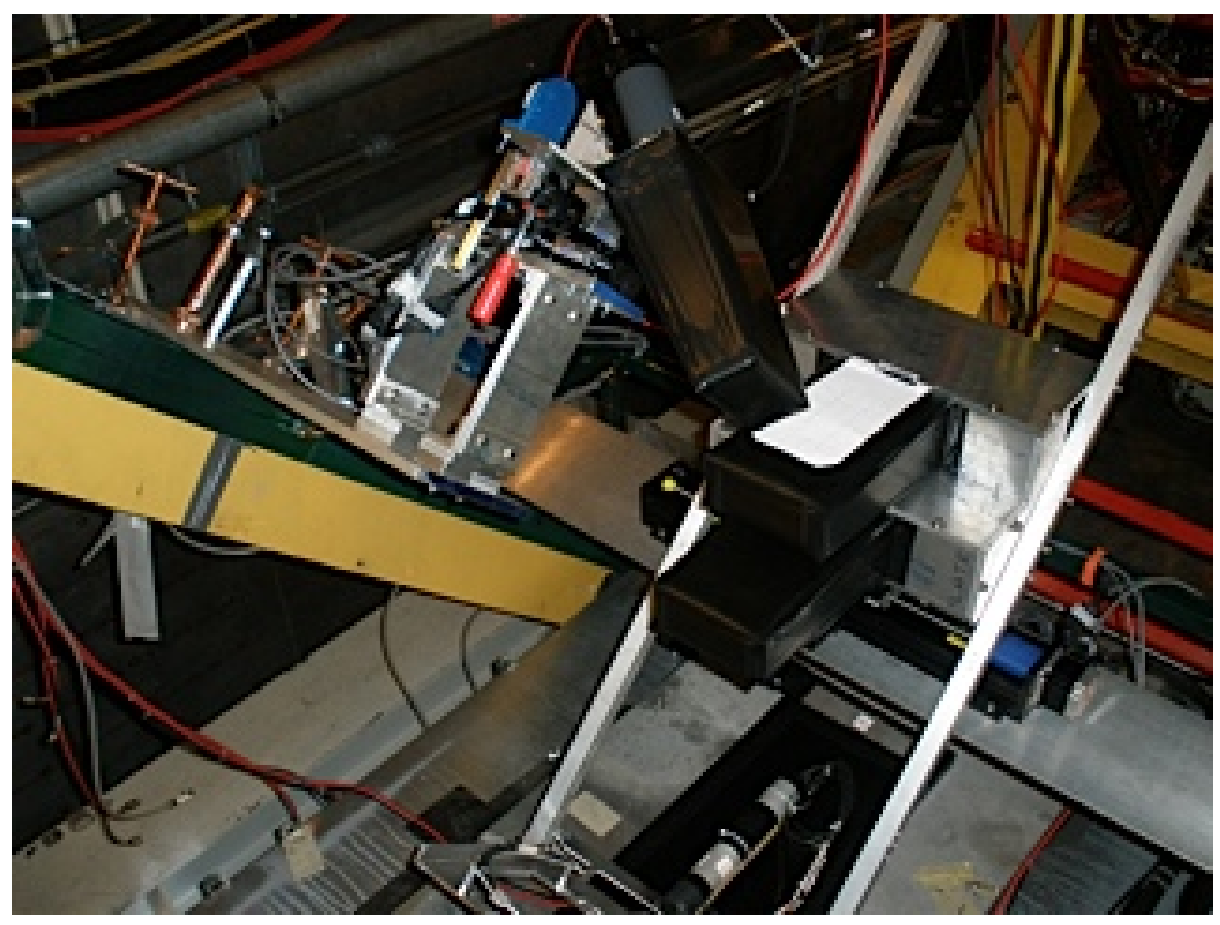

Figure 3.10: Final detector assembly in one of the spectrometers. The $A_{T}$ detector is set up on the yellow beam on the left. The $A_{T}$ and the focal plane detectors are placed on remotely controlled $\mathrm{x}$ - and $\mathrm{y}$ - tracks to facilitate proper alignment with the appropriate signals.

an aluminum lightguide and a 2-inch PMT used to integrate the Cherenkov light.

Figure 3.10 is a picture of the final configuration of all three detectors in one of the spectrometer arms. The $A_{T}$ and focal plane detectors were mounted on tracks that could be controlled remotely, used to ensure proper alignment. Also, each detector had a two LED system installed near the PMT base for use in linearity tests. Both LEDs were outfitted with attenuators, one of them $10 \%$ stronger than the other. By varying the voltage driving the main LED, and keeping the voltage of the $10 \%$ LED constant, linearity can be measured by studying any variations in the difference of the two signals.

The PREx focal plane detectors were designed to get a high electron count and maximize their resolution by optimizing the thickness of the quartz. While the resolution gets better by making the quartz thicker, this leads to a large Landau tail caused by ionization of delta rays generated in the material, which in turn can increase the RMS of the signal. If the quartz is too thin, the detector resolution is poor 


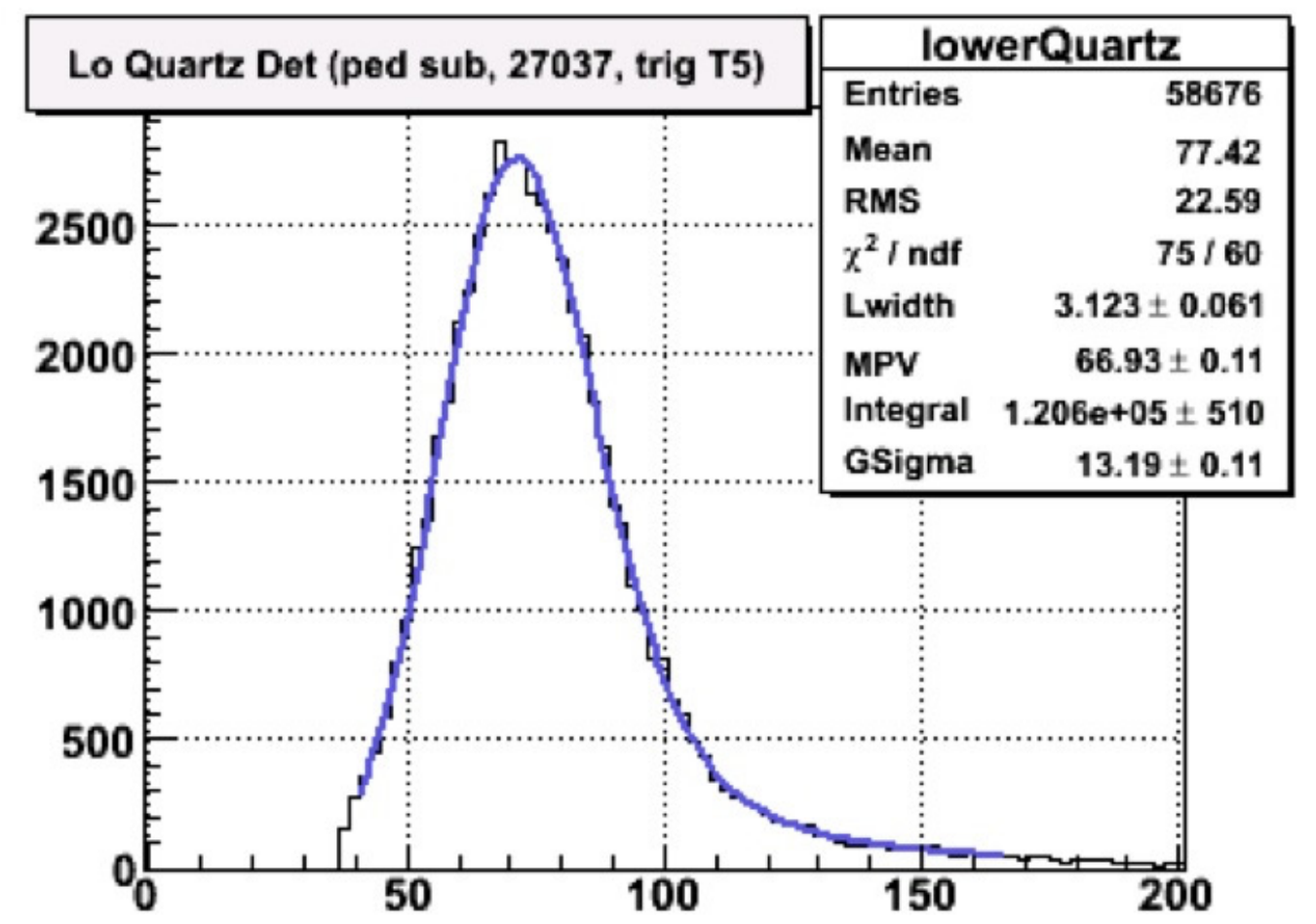

Figure 3.11: Signal profile for one of the 'thin' quartz detectors, referred to in this plot as lowerQuartz. By dividing the RMS by the mean of the histogram fit, one gets the energy resolution $\sigma_{E} / E$ of the detector.

but the Landau tail is minimized. For this reason, the thickness must balance these two effects. The two thicknesses chosen for the final detector design $(0.5$ and $1.0 \mathrm{~cm})$ ended up achieving similar resolutions.

Figure 3.11 shows a profile of the electron signal measured by one of the 'thin' quartz detectors. From this profile, one can obtain the energy resolution, defined as $\sigma_{E} / E$, to be $\approx 29 \%$. This value is important because the statistical noise of the pair-wise asymmetry gets blown up by a factor $\sqrt{\left(1+\left(\sigma_{E} / E\right)^{2}\right)}$. In this case, the statistical noise only gets degraded by a few percent due to the energy resolution.

\subsection{Higher Resolution Analog to Digital Converters}

The previous HAPPEx experiments used 16-bit ADCs to read out integrated detector signals. For PREx, we wanted an even higher level of precision than previous parity 


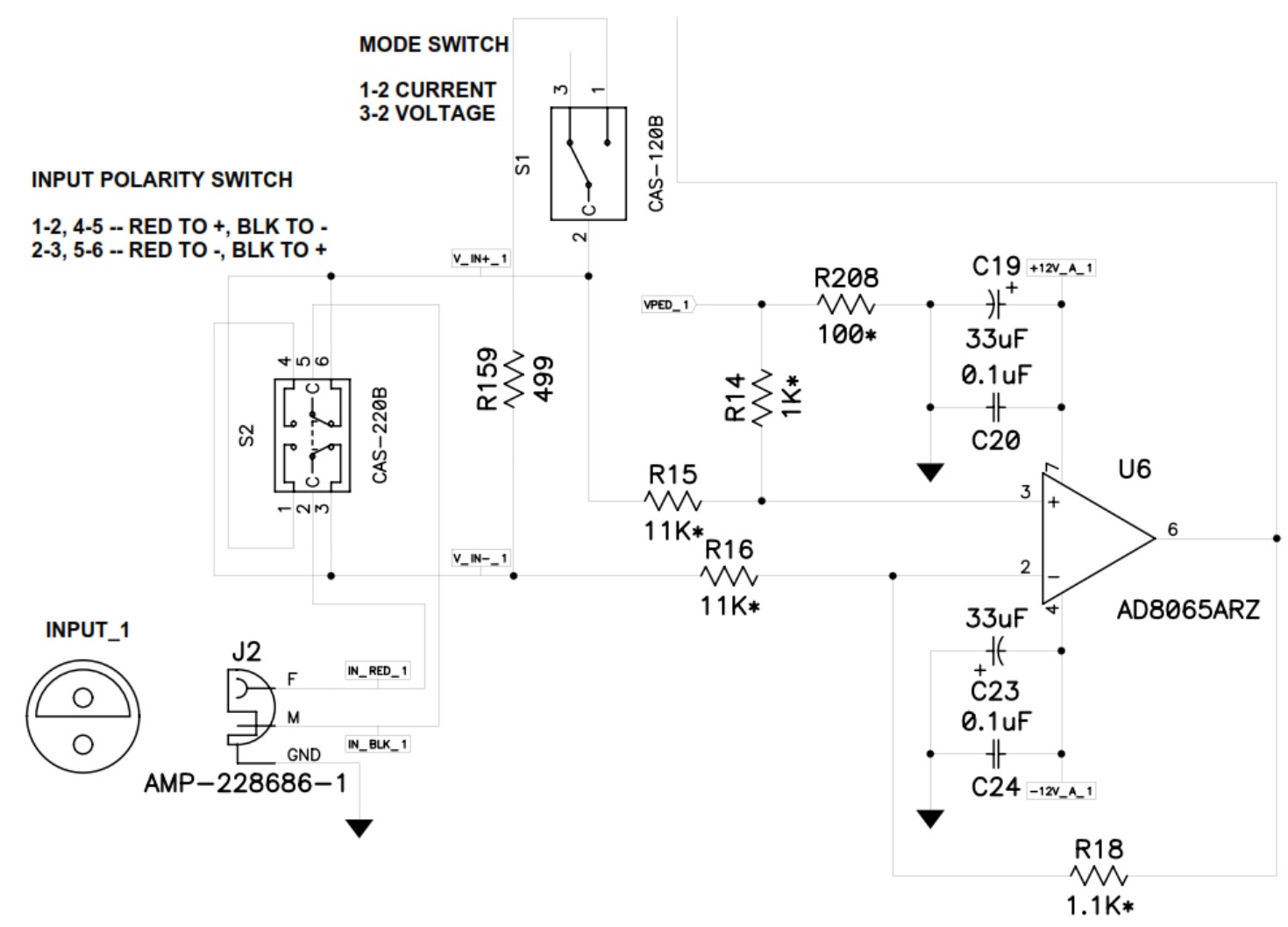

Figure 3.12: Partial circuit diagram of one of the input channels on an 18-bit ADC. The resistor labeled R159 was changed to adjust the maximum current range.

experiments. The 18-bit ADCs were developed in part to help improve noise contribution from pedestals by a factor of 4 . While HAPPEx ran with a helicity frequency of $30 \mathrm{~Hz}$, PREx ran with a frequency of $120 \mathrm{~Hz}$, requiring the ADCs to integrate the data four times faster. These new ADCs were designed by Fernando Barbosa (Fast Electronics Group) and Edward Jastrzembski (Data Acquisition Group) from the Jefferson Labs Physics Division.

These ADCs were designed so that they could be used to read in both voltage and current signals. A voltage-mode ADC was produced to be used for BCMs and BPMs, voltage signals that typically vary from 0 to $+5 \mathrm{~V}$. A current-mode ADC was produced to read in PMT signals from main detectors and luminosity monitors. The difference between the two modes is the value of three resistors on the front end, labeled R14, R16 and R159 in Figure 3.12, which shows a circuit diagram of an ADC input channel. Their values can be modified according to the strength of the expected output voltage and current signals. Adjusting R14 and R16 will change the 
range of the voltage mode, while the resistor labeled R159 was changed to modify the maximum range of the current-mode channels.

The maximum range of these new ADCs can additionally be adjusted by using two gain level settings, referred to as Integration and Conversion gain, Int and Conv for short. Making adjustments to the Int gain, which can range from 0 to 3 with 0 as the maximum value, changes how certain capacitors are used to integrate the signal. Similarly, adjusting the Conv gain, which can range from 0 to 15 with 15 as the maximum value, changes the amount of resistors used to convert the signal. These can be combined to produce up to 64 individual gain levels.

Before any official use, all the 18-bit ADCs were tested extensively (at 30Hz) for pedestal noise, differential linearity, crosstalk and linearity with respect to sampling rate, signal strength and integration time. The results of several of these tests will be discussed thoroughly in this section and some other details can be found in [25].

\subsubsection{Pedestals}

The pedestal of an ADC channel is the output produced when there is no input signal connected. This value must be calibrated to a small positive value before taking any data so it has minimum effect on the maximum signal range and can later be subtracted out during data analysis. While testing, we found that the pedestal values depended on the gain level settings. To compensate for this, 12-bit DACs (digital to analog converters) were installed on every ADC channel to produce a voltage offset that keeps the pedestal signal at a small value. A database of DAC values for all the 18-bit boards was created so that every time a gain setting was changed, the pedestal was automatically re-calibrated.

Since the expected statistical precision during the experiment was about $125 \mathrm{ppm}$, it was desirable to have a much smaller contribution from pedestal noise. When optimizing for minimum pedestal noise, we looked at several values, defined below.

- Open-Circuit Pedestal Difference is the pair-difference in ADC values with nothing plugged in, expressed in channels.

- Pedestal Difference in channels is the pair-difference in ADC values with signal plugged in, expressed in channels. 


\begin{tabular}{|c|c|c|c|}
\hline Gains & Gain Factor & Ped Diff(CH) & Ped Diff $(\mathrm{ppm})$ \\
\hline$(3,0)$ & 1 & 3.45 & 8.61 \\
\hline$(2,0)$ & 1.58 & 4.02 & 10.05 \\
\hline$(3,1)$ & 2 & 4.38 & 10.94 \\
\hline$(1,0)$ & 2.17 & 4.2 & 10.51 \\
\hline$(3,2)$ & 2.9 & 5.85 & 14.63 \\
\hline
\end{tabular}

Table 3.2: Pedestal Noise for the lowest gain levels with no signal connected. The value given in the last column is the pedestal difference divided by twice the ideal signal strength, 200K.

- Pedestal Difference in ppm is the pair-difference divided by the sum of observed ADC values (ideally about $200 \mathrm{~K}$ ) with signal plugged in, expressed in ppm.

It was found that the open-circuit pedestal difference depends only on the gain settings used, and is optimized by using the lowest Int and Conv gain levels. Table 3.2 shows the open-circuit pedestal difference for the lowest five gain settings. The data clearly shows that the pedestal noise gets worse with increasing gain factor. During PREx, only the lowest gain levels were used. An upper limit of $20 \mathrm{ppm}$ was set for any ADC channels used to read out the detector signals.

Further testing included investigating the pedestal difference when there was a current source connected to the ADC channel. The current source originated from a simple PMT and LED test setup. The current was increased in steps by adjusting the high voltage source of the PMT. This test was conducted at the minimum gain settings to take advantage of the board's maximum usability range. The results are shown in Figure 3.13, which shows that as the current is increased, the pedestal difference in ppm settles to a minimum value of about $35 \mathrm{ppm}$, which corresponds to 14 channels in an 18-bit ADC. This means that as the signal increases, the pedestal noise contribution goes up by the same ratio.

\subsubsection{Differential Linearity}

When digitizing data, there is a reduced probability that a hit will be produced at certain bit locations. Figure 3.14 shows the signal being produced by an ADC channel with a varying current connected. The gaps shown always occur at the same ADC 


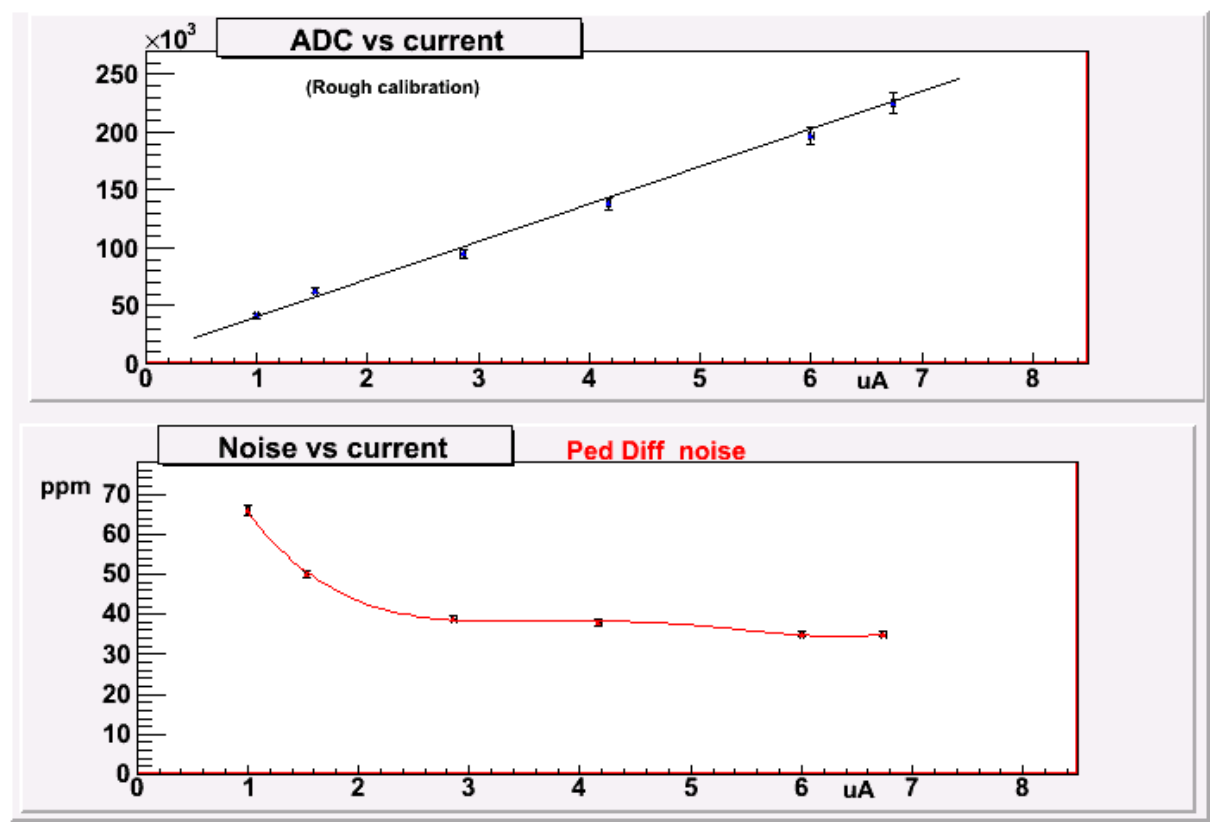

Figure 3.13: Pedestal differences in ppm as the input current is increased.

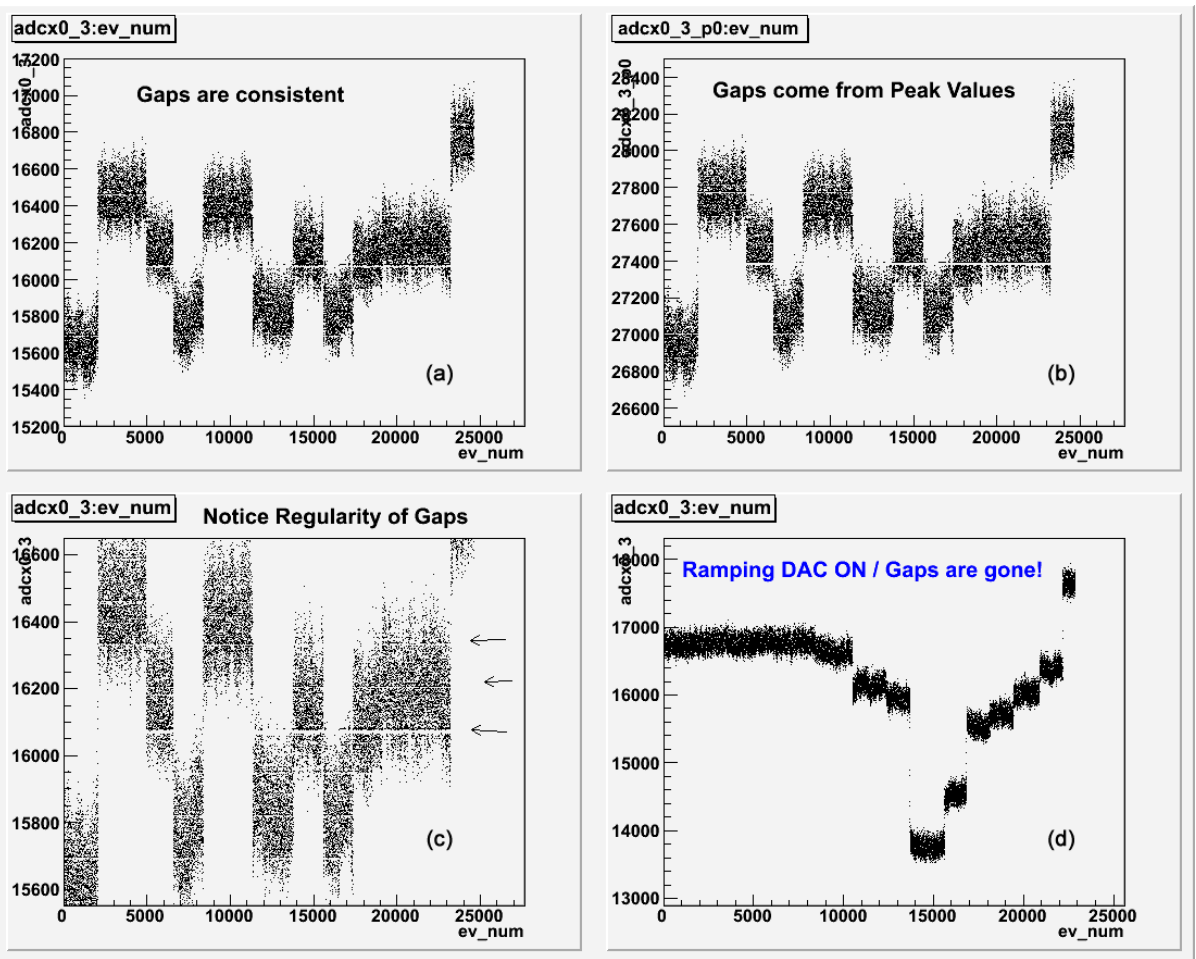

Figure 3.14: Plots (a), (b) and (c) show gaps in the data consistent with missing bits. These gaps go away when DAC is used (d). 

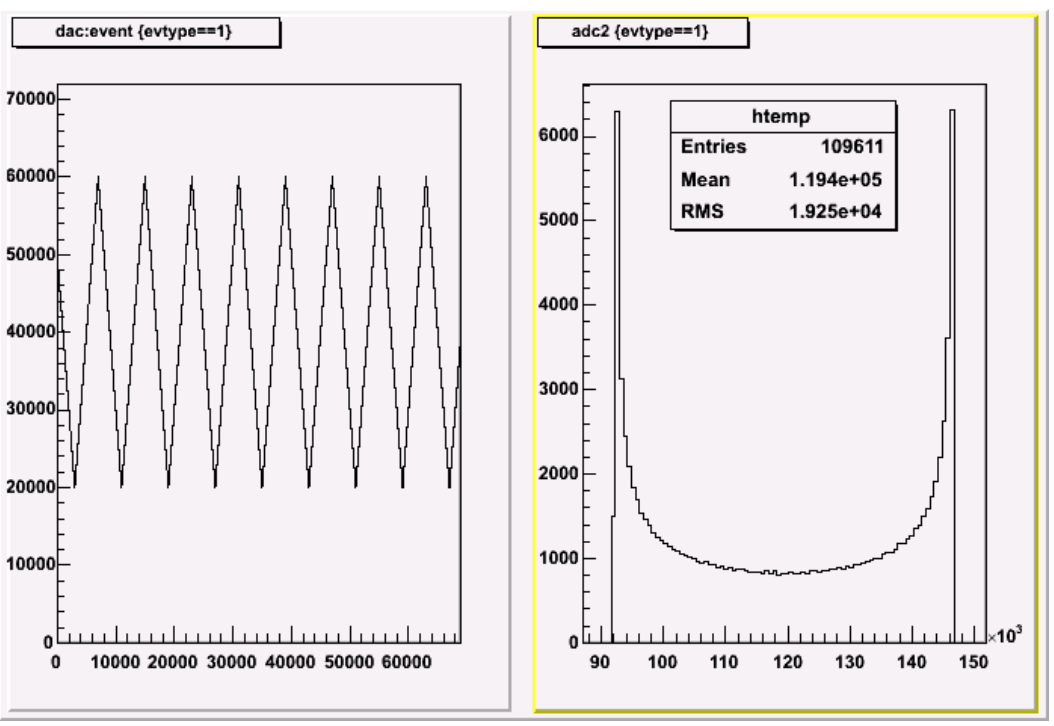

Figure 3.15: When using a slowly varying DAC (left), the histogram of a sine wave is a smooth secant distribution (right).

value and are an effect of the ADC's resolution. To smooth out these gaps, DAC noise is added to each integrated sample of the ADC. DAC noise is defined as a slowly varying or random value that is added during digitization and later removed during data analysis. The bottom right plot in Figure 3.14 shows the effect of the DAC noise after its subtraction, completely removing all the gaps.

Another test used to verify good differential linearity involved using a function generator to create a slowly varying voltage signal. The signal produced by the function generator was a slowly varying sine wave. The left plot of figure 3.15 shows the DAC signal applied when reading out the sine function. As expected, the plot on the right shows a very smooth secant distribution as a result of using DAC noise.

\subsubsection{Cross-Talk}

Another phenomenom that was tested for was cross-talk, which occurs when a large signal, connected to one ADC channel, has an unwanted effect on a neighboring channel. Figure 3.16 shows the results of one of our cross-talk tests. The top plot shows the pedestal of an ADC channel. The bottom plot shows the same signal when a pulser has been connected to an adjacent channel. The pulser signal has no effect on the adjacent channel, indicating no cross-talk. Several other tests were conducted to 


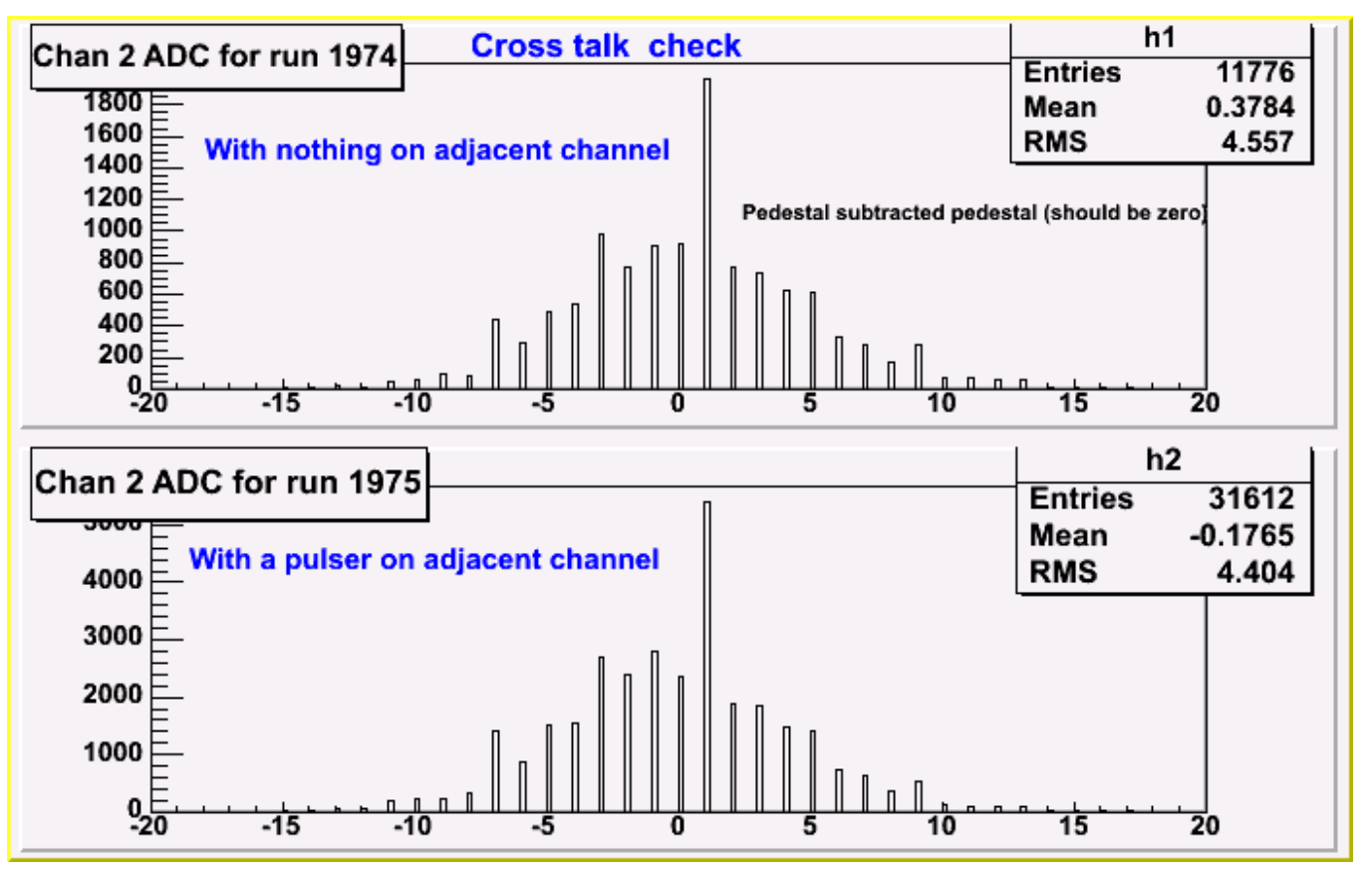

Figure 3.16: The top plot shows the pedestal signal from one of the 18-bit ADC channels. The bottom plot shows the same channel after a pulser signal has been connected on an adjacent channel. No significant effect is observed.

make sure that the helicity signals did not produce any cross-talk, a problem that was observed during previous experiments in Hall A. Making sure all the electronics used in the experiment are properly grounded is essential in avoiding cross-talk effects.

\subsection{Detector Linearity}

One of the most essential parts of our Luminosity Monitors (lumis) and main detectors are the photomultiplier tubes (PMTs) used to integrate the signals we are interested in. The PMTs used for the lumis and for the PREx detectors were model Hamamatsu R7723, with a bialkali photocathode and a 2-inch diameter. They are most efficient (26\% quantum efficiency) for wavelenghts of $420 \mathrm{~nm}$ and have typical gains between $10^{3}-10^{6}$ for its recommended operating range.

The PMTs used for the HAPPEx detectors were 5-inch diameter Burle 8854 tubes. They have a bialkali photocathode of high efficiency, $22.5 \%$ at $385 \mathrm{~nm}$, and much higher gain levels of $10^{4}-10^{8}$. 


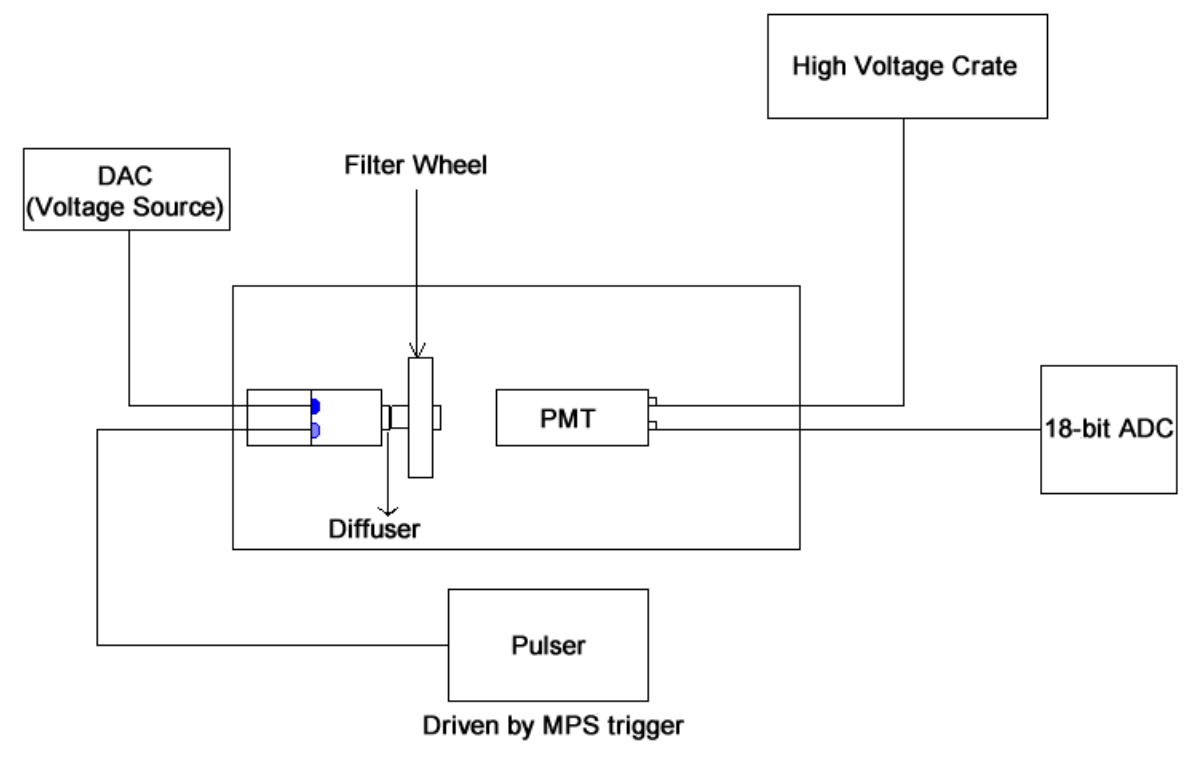

Figure 3.17: Black box setup for PMT linearity studies.

In order to use these PMTs properly, their usability range must be studied thoroughly to avoid any nonlinearities in their signal output. For this purpose, an experimental test setup was created using a light-tight box and the HAPPEx DAQ. About a dozen PMTs were tested using this setup and a good linearity range was found for all of them.

\subsubsection{Experimental Test Setup}

Figure 3.17 is a schematic showing the components used for the test setup. The PMT, filter wheel and LEDs were kept inside a black toolbox which was tested for light leaks. Figure 3.18 shows a picture of the black box setup during tests with a 5-inch Burle PMT. Each phototube is studied at different gain levels by varying the amount of light hitting the photocathode in discrete steps. The output signal of the PMT is then read out using the HAPPEx integrating DAQ and examined with our analysis software.

To first order, a PMT can have a non-linear response to a signal $N$ that can be expressed as

$$
N_{P M T}^{ \pm}=N^{ \pm} \times\left(1+\beta N^{ \pm}\right)
$$




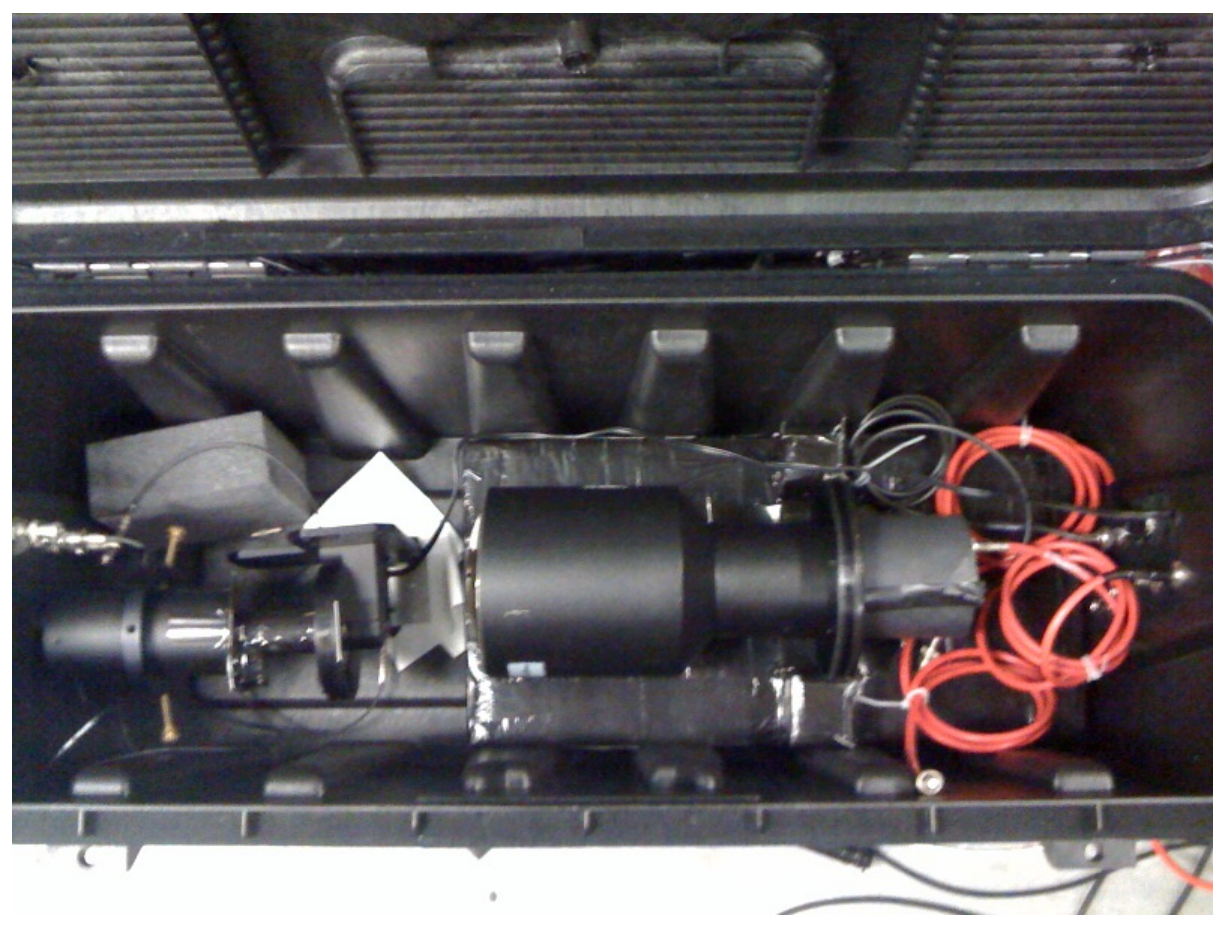

Figure 3.18: Picture of the linearity test experimental setup. PMT shown was 5-inch Burle used in HAPPEx-III.
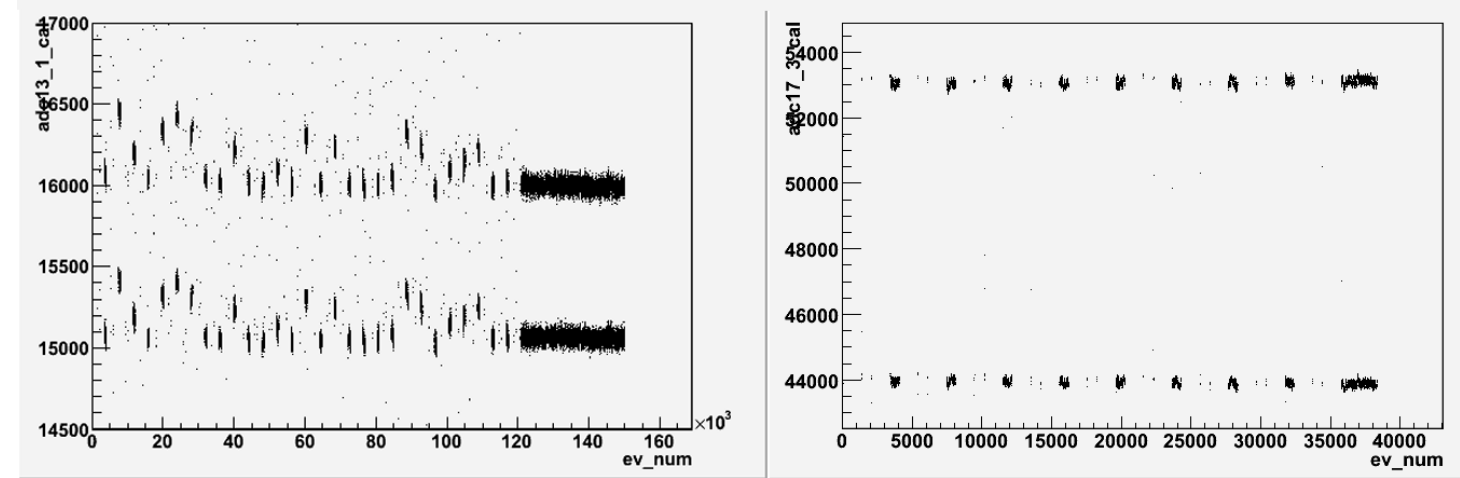

Figure 3.19: Plot on the left shows how the signal varied when the filter returned to the same setting before installing a small aperture. On the right, with the aperture, the signal is now stable. 


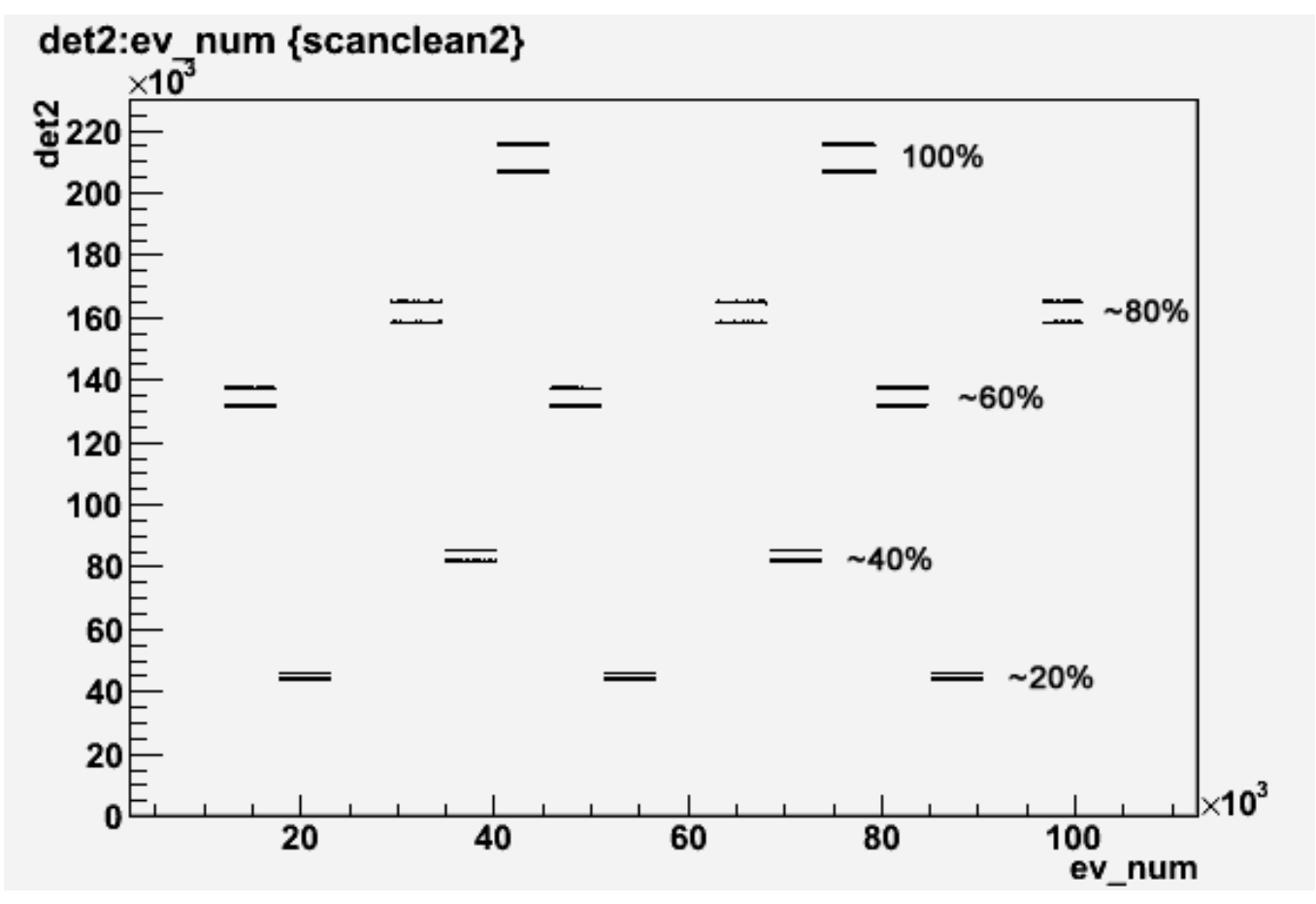

Figure 3.20: Sample raw data from linearity test. The signal is pseudo-randomly varied between six filter settings.

where the \pm superscript corresponds to the two helicity states of the signal. In our setup, we will simulate these two states by using two LEDs, one kept at a constant light level, and the other pulsing at the same rate that the helicity signal will change during the experiment. The constant LED is meant to simulate the continuous signal we expect from the PMT when at nominal running conditions. The pulsing LED toggles on and off so that an asymmetry can be calculated between adjacent helicity windows. The high signal $N_{+}$will end up being the sum of the constant, or baseline LED, and the pulsed LED.

For linear behavior, as the light is attenuated by a certain factor, the value of the asymmetry being read out by our DAQ should remain constant. For our tests, the LED light was attenuated by using an automated filter wheel with six transmission settings: 0, 20, 40,60, 80 and 100\%. The two LEDs were housed in a cylinder with a small aperture (1 cm diameter) on one side. While testing, we found that making this aperture small, as well as using a diffuser, minimized any random variations of the light seen when taking subsequent readings at specific filter settings (see Figure 3.19).

Linearity tests were conducted at several light levels in a wide range of High 

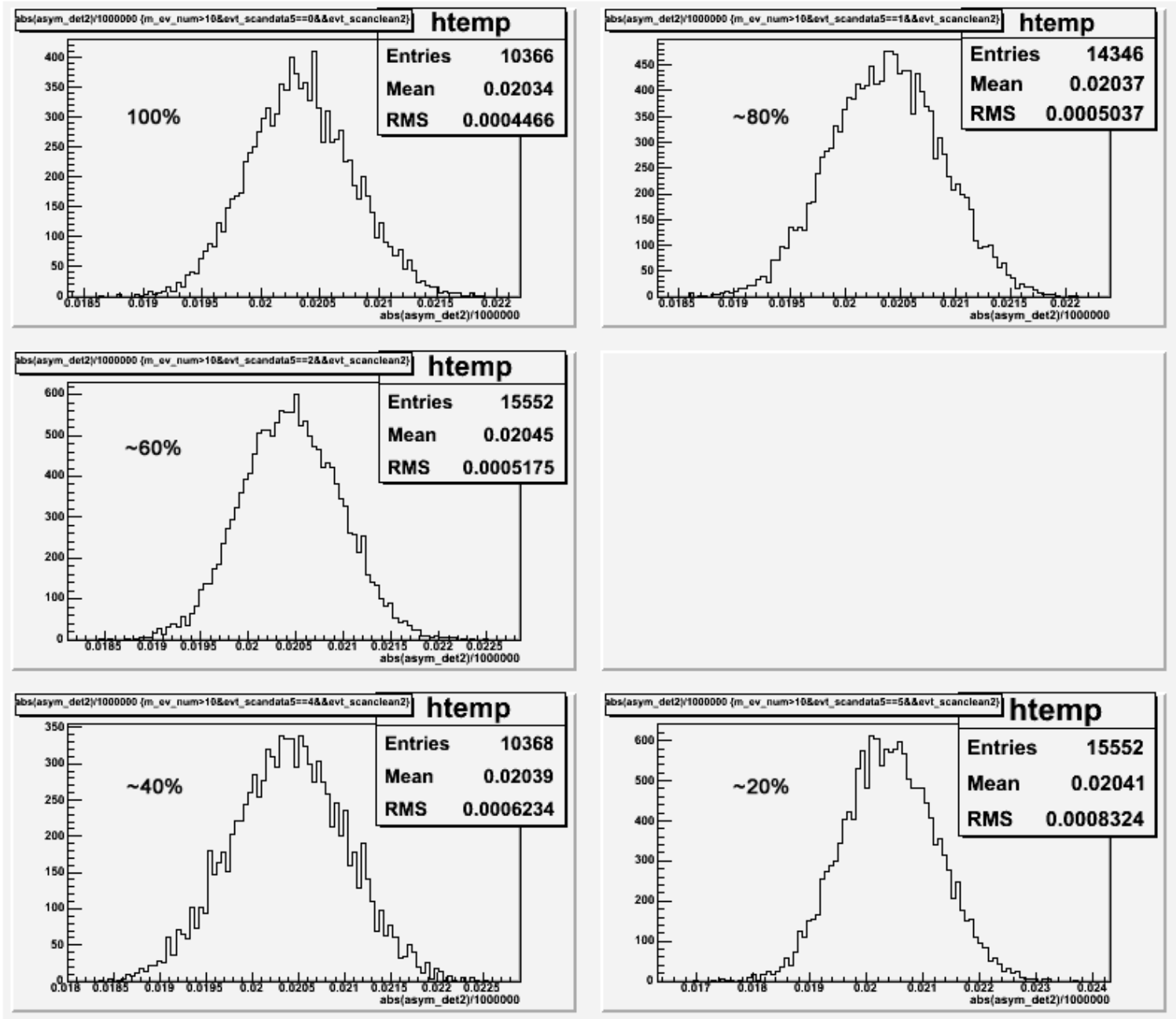

Figure 3.21: For the linearity tests, asymmetries are calculated for each filter attenuation and analyzed for any variations. 


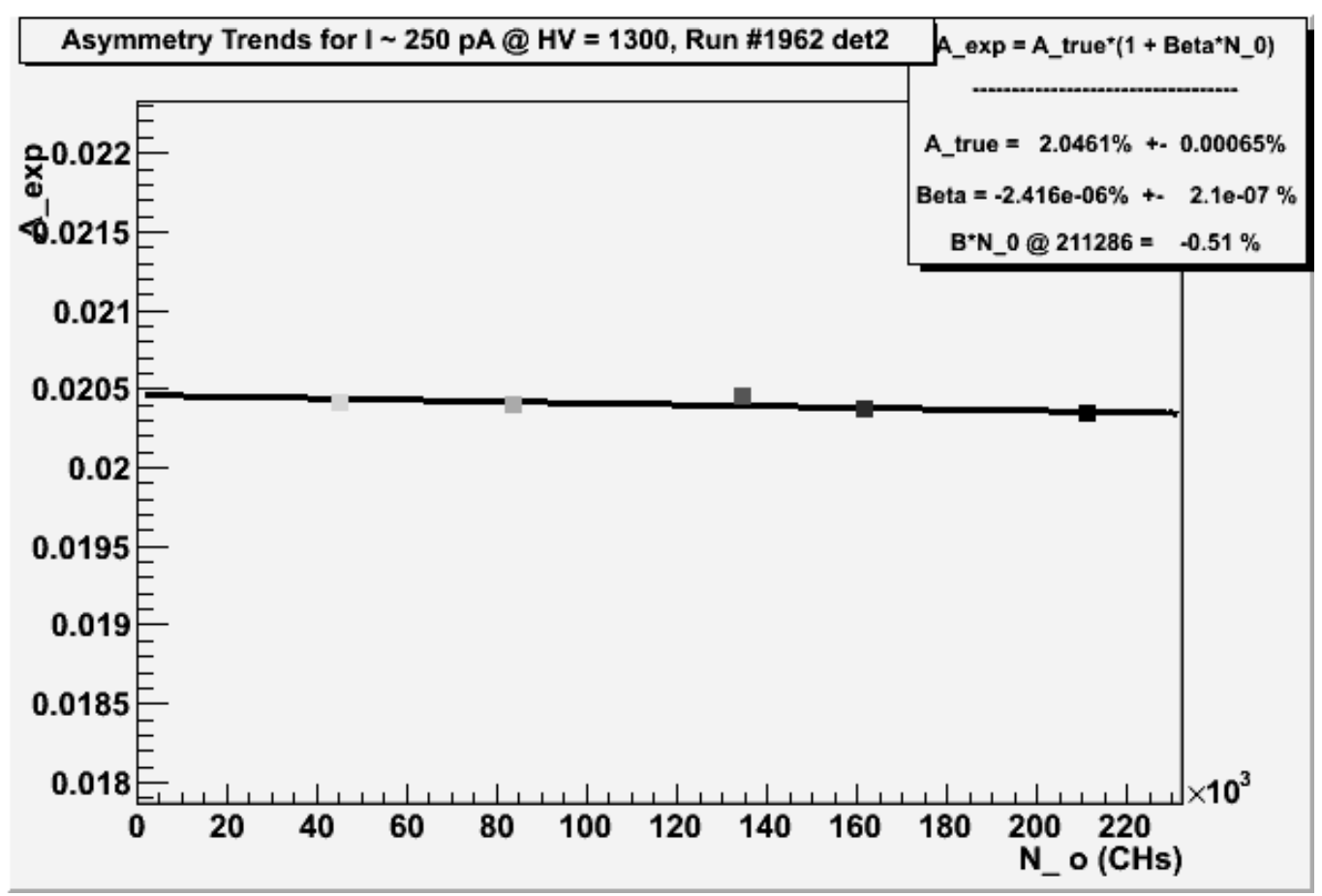

Figure 3.22: By fitting the measured asymmetries with respect to the average of $N^{+}$and $N^{-}$, one can extract a slope that represents the degree of nonlinearity for these running conditions.

Voltage values. The goal was to find the usability range of the PMT at which it was linear to better than $2 \%$. At each setting, the filter wheel sampled each transmission setting three times in a pseudo-random pattern. Figure 3.20 shows the raw signals measured during one of the linearity test runs. From this data, asymmetries are calculated for all five filter settings, as shown in Figure 3.21.

Using Equation 3.1, the non-linear effect on the raw asymmetry is found to be

$$
A_{\text {exp }}=\frac{N_{P M T}^{+}-N_{P M T}^{-}}{N_{P M T}^{+}+N_{P M T}^{-}} \approx A_{\text {true }} \times\left(1+\beta N_{0}\right)
$$

where

$$
N_{0}=\frac{N_{P M T}^{+}+N_{P M T}^{-}}{2}
$$

By plotting $A_{\text {exp }}$ vs. $N_{0}$, we can easily extract a slope equal to $A_{\text {true }} \beta$ that will determine how non-linear the phototube is for a specific light level and gain setting, dependant on the HV applied. Each test was run at least three times to verify the reproducibility of the results. 
Figure 3.22 shows how we extract the degree of nonlinearity from the data shown in Figure 3.21. This test was conducted with one of the PREx detector PMTs right before being installed in Hall A, and was designed to simulate the expected input signal during the experiment. The results show that this PMT has very good linearity for these running conditions. In Appendix A, a variety of test results will be discussed in more detail. They will show how such tests gave us an understanding about how to avoid large non-linearities with our detector and lumi PMTs.

Overall, the tests showed that as the input photocathode current of the PMT becomes much higher than 10-20 nA, it is increasingly difficult to find regions of good linearity. For low enough input currents, we found that there was a range of PMT gains (value of high voltage source) that showed linear behavior better than $2 \%$. We established that we could generally have good linear behavior for output currents of 20-40 $\mu \mathrm{A}$. For this reason, the ADCs used to read out PMT currents were specially designed to have the proper dynamic range. 


\section{CHAPTER 4}

\section{HAPPEX-III ANALYSIS}

The HAPPEx-III experiment took place in the Fall of 2009 between August 19 and October 27. After 70 days of production running, the experiment was able to collect 193.6 Coulombs of charge at a beam current of $100 \mu \mathrm{A}$, about $77 \%$ of the proposed goal. This amount of data does not include time spent doing calibration runs and other systematic studies such as linearity tests and polarization measurements.

Each production run was about an hour long and produced a maximum of 54,000 asymmetry pair calculations. Data was taken at different IHWP states, which was changed every 24-48 hours. All the data collected during each variation of the IHWP, usually about a million good pairs, is grouped into slugs. The total data collected throughout the experiment corresponds to 28 slugs.

This chapter will describe how a physically meaningful value of the parity-violating asymmetry is extracted from the data collected. To do so, the raw asymmetry must be found and corrected for beam systematics, s and polarization.

\subsection{Overview}

The asymmetry measured with the HAPPEx detectors must undergo several analysis tasks before the physics asymmetry of the parity-violating interaction can be obtained. While the detectors measure $A_{\text {raw }}$, what we are interested in is the following value

$$
A_{\text {phys }}=\frac{K}{P_{b}} \frac{\left(A_{\text {raw }}-F-T\right)-P_{b} \Sigma_{i} A_{i} f_{i}}{1-\Sigma_{i} f_{i}},
$$

where $K$ is a factor that accounts for the finite kinematic acceptance, $P_{b}$ is the degree of beam polarization, $F$ is the false beam asymmetry correction, $T$ is the transverse asymmetry correction, $f_{i}$ are the background fractions and $A_{i}$ are the asymmetries associated to the background processes. 
The raw asymmetry analysis is performed with a 'blinded' offset. This means that each asymmetry calculation has a random offset applied to it that is significantly larger than the statistical error of the expected result. All data analysis tasks are completed before this offset is removed. This offset is meant to add uncertainty to the final result until all the corrections to the raw asymmetry have been done properly, so that the final physics asymmetry is not known until the group is ready to make the result available to the public.

\subsection{Data Selection}

The analysis of the data was performed in parallel by groups at the University of Massachusetts and University of Virginia. The analysis is performed using a software package specially designed for these parity experiments known as PAN (Parity ANalyzer). PAN is written in the $\mathrm{C}++$ programming language and uses the ROOT framework for plotting. ROOT is an object oriented program and library developed by CERN (European Organization for Nuclear Research) for use in particle physics data analysis [30].

The first step in the asymmetry analysis is to determine several data quality cuts used to get rid of 'bad' data. Generally, bad data can be defined as data collected when the beam was off or when any of our electronics are saturated or malfunctioning in some other way. Another type of cut accounts for any sudden jitter in the beam monitor signals. Table 4.1 shows a summary of the threshold cuts used on the beam current and position monitors, as well as the number of helicity windows taken out before and after the bad data. Other more specific cuts that do not depend on beam parameters are also made. All the generic cuts used for the entire data set are now defined:

- Low Beam: A beam current monitor (BCM) signal below 22000, corresponding to about $75 \mu \mathrm{A}$, was a reasonable cutoff to account for beam trips and ramps. This cut also gets rid of any runs done at low currents that can make the asymmetry widths much bigger. While only 10 events are cut out before the signal drops below the cutoff, 40 windows are cut once the full signal returns. This is to avoid any instabilities or non-linearities in the monitors and detectors due to the process of beam recovery. 


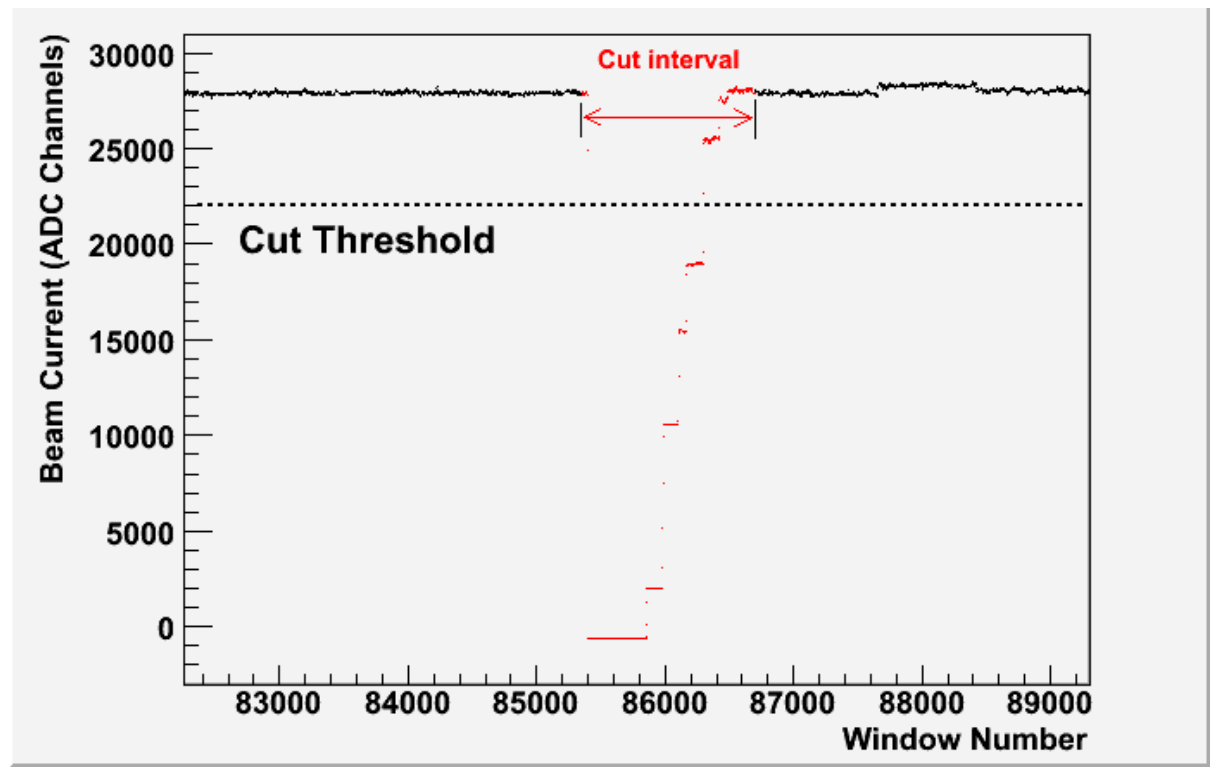

Figure 4.1: This beam current monitor (BCM) data is from a run with a beam trip. The data in black is usable data, while the red data is what was cut out before the analysis was done. All data below 22000, as well as 10 windows before and 40 windows after was marked as 'bad' data.

\begin{tabular}{|c|c|c|c|}
\hline Cut & Threshold & Extent lo & Extent hi \\
\hline bcm 1 & $2 \mu \mathrm{A}$ & 10 & 40 \\
\hline bpm4ax & $200 \mu \mathrm{m}$ & 50 & 50 \\
\hline bpm4ay & $200 \mu \mathrm{m}$ & 50 & 50 \\
\hline bpm4bx & $200 \mu \mathrm{m}$ & 50 & 50 \\
\hline bpm4by & $200 \mu \mathrm{m}$ & 50 & 50 \\
\hline bpm12x & $200 \mu \mathrm{m}$ & 50 & 50 \\
\hline
\end{tabular}

Table 4.1: Summary of threshold cuts and intervals used to remove unwanted data. Extent values refer to the number of windows that have been cut from the data before (lo) and after (hi) an unwanted event. 
- Burp Cut: This threshold cut accounts for when a beam current monitor signal changes by a certain value caused by fluctuations in beam intensity. The value shown for bcm 1 in Table 4.1 corresponds to a $2 \%$ change in the signal when beam current is $100 \%$.

- Monitor Saturation Cut: At several times during the experiment the raw signal of one of the beam position monitors, referred to as bpm12, was saturated. This was due to malfunctions in the bpm12 feedback electronics after a beam trip, allowing the signal to be at a higher gain setting than is usually used, leading to signal saturation. Any events with signal higher than 132000 channels, as well as 30 windows before and after, were purged by this cut.

- Position Monitor Burp Cut: This is the threshold cut used for the beam position monitors. Any large beam excursions can cause detector rates to fluctuate, leading to detector non-linearities. For this reason, this cut gets rid of excursions higher than $200 \mathrm{~nm}$, as well as 50 windows before and after.

- ADCX DAC Burp Cut: This cut is specific to signals from the 18-bit ADCs, referred to as ADCX. These events are corrupted by certain internal ADC errors related to large jumps in $\mathrm{DAC}$ values.

- Event Sequence: Any events that fail synchronization tests between the readout helicity and the helicity expected by the PAN software are marked as bad.

- Pair Sequence: To calculate a meaningful value, the asymmetry analysis requires that pairs of events have the opposite helicity. When this is not the case, that pair of events, as well as 25 before and after, are cut. While this cut is not very common, it is important to get rid of events before and after to not create any bias against a particular helicity state.

- ADCX Bad: Any other data that might have been corrupted by internal ADC errors on the 18-bit ADCs. These errors are extremely rare and were usually caused by miscomunication between the DAQ system and an individual electronics crate.

The cuts listed are performed by the analysis software and eliminate the data before calculating the pair-wise asymmetries. Once the whole data set has been analyzed, some extra cuts will need to be applied. One of these extra cuts is used on data 

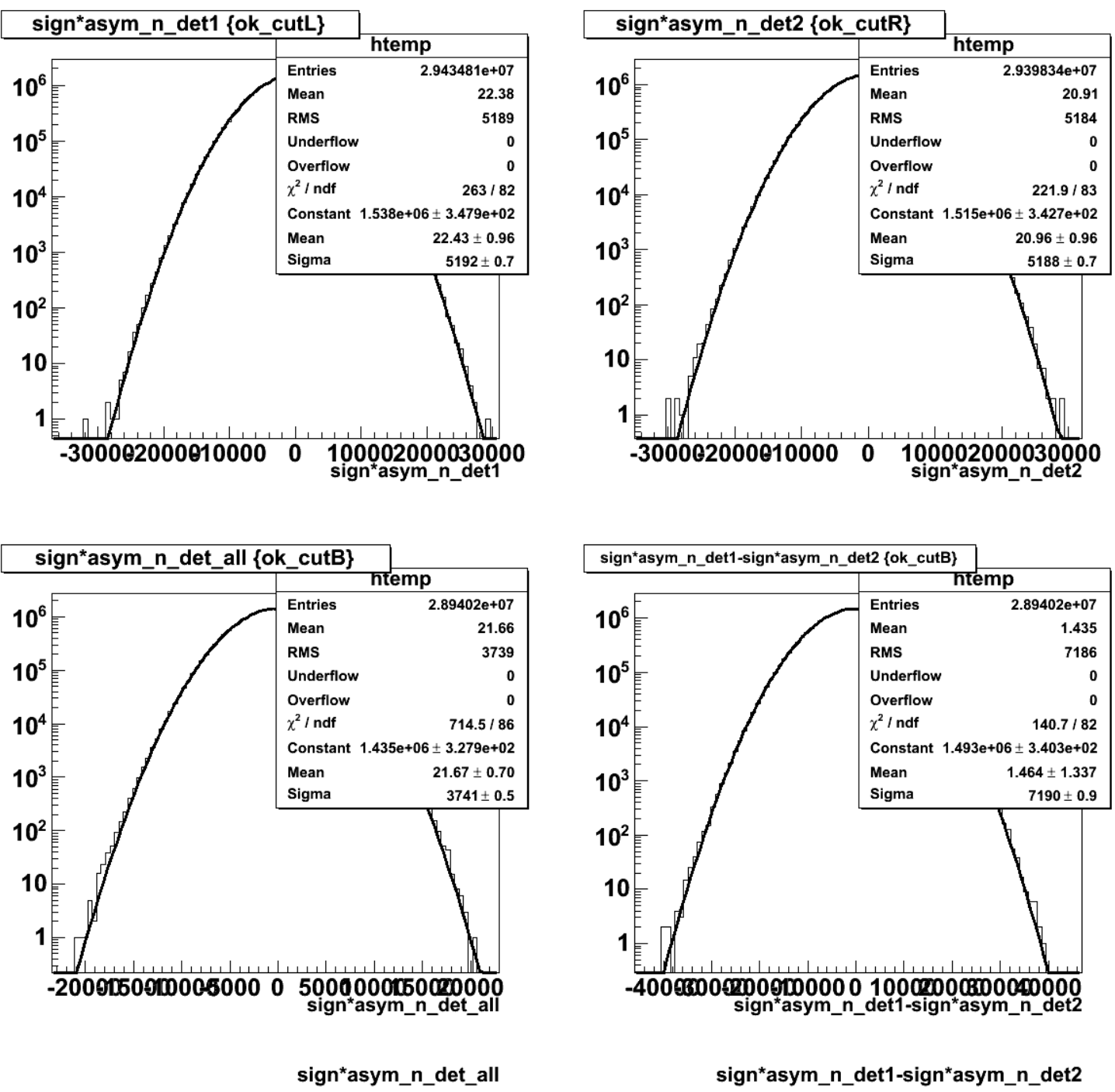

Figure 4.2: Sign-corrected asymmetries, in units of parts-per-million, calculated for all data that passed the most basic data quality cuts. Modulation data is included. 
from each individual spectrometer, dependent on whether the magnets were behaving properly or not. There were times when one of the spectrometer magnets stopped working, preventing the elastically scattered particles from reaching the detector focal plane. This data is not useful for calculating asymmetries and is therefore discarded as well.

\subsection{Raw Asymmetry}

Once the data has passed all quality cuts, the next step in the analysis is to find a raw asymmetry. This is done by finding the asymmetry of the normalized detector flux

$$
A_{\text {raw }}=\frac{D^{R} / I^{R}-D^{L} / I^{L}}{D^{R} / I^{R}+D^{L} / I^{L}},
$$

where $D$ is the integrated detector flux, $I$ is the integrated current as measured by one of the beam current monitors and $R(L)$ denotes the state of the beam helicity. Asymmetries are found for each helicity pair and the averages for each run are examined to make sure that the data has a gaussian profile.

Figure 4.2 shows histograms of the calculated raw asymmetries for both detectors. There are two main HAPPEx detectors, one in each HRS. Det1 is located in the LHRS and det2 in the RHRS. Also plotted is the combination of both detectors det all as well as their difference. The data in these plots was also corrected for the different IHWP states. There is slightly less data for the RHRS detector det2 because of bad spectrometer magnet settings. If the magnetic field is incorrect or is completely turned off, the elastic peak does not reach the focal plane of the detectors.

When calculating the detector combination det all, proper weighing of each individual detector should be used to take into account any differences in the detected flux. These differences in rate affect the width of the asymmetry distribution $\sigma_{k}=1 / \sqrt{N_{k}}$, which depends on the number of electrons incident on the detector $N_{k}$. For this reason, a weight factor of $w_{k}=1 / \sigma_{k}^{2}$ is used on each detector signal. The weight factors should also be normalized to one so that for two detectors,

$$
w_{k}^{\prime}=\frac{w_{k}}{w_{1}+w_{2}}
$$


The det all raw asymmetry is calculated by using the following expression

$$
A_{\text {det }}^{\text {all }}=\frac{\left(D_{1}^{R} w_{1}^{\prime}+D_{2}^{R} w_{2}^{\prime}\right)-\left(D_{1}^{L} w_{1}^{\prime}+D_{2}^{L} w_{2}^{\prime}\right)}{\left(D_{1}^{R} w_{1}^{\prime}+D_{2}^{R} w_{2}^{\prime}\right)+\left(D_{1}^{L} w_{1}^{\prime}+D_{2}^{L} w_{2}^{\prime}\right)} .
$$

When the two detector signals are combined to form det all, the statistical width goes down by a factor of $\sqrt{2}$. From Figure 4.2 , we see that the RMS of the Det1/Det2 asymmetry was 5192/5188 ppm. The RMS value of the det all asymmetry should be $\approx 3670 \mathrm{ppm}$. The measured RMS for det all was actually $3741 \mathrm{ppm}$, pointing to extra sources of noise of $\approx 725 \mathrm{ppm}$. The extra noise can be attributed to boiling effects and other sources of common mode noise.

\subsection{False Asymmetry Corrections}

The raw asymmetry must now be corrected for any helicity-dependent false asymmetries that are unrelated to the parity violating measurement we are interested in. The main source of these false asymmetries comes from helicity-correlated differences in the properties of the beam. Other contributions can come from electronics pickup in the DAQ signals, as well as from interactions between the target and the vertical component of the beam polarization.

For a parity violation experiment, it is essential that all the properties of the polarized beam are the same for both helicity states. As part of the analysis, a charge asymmetry and several position differences are calculated using measurements made by BCMs and BPMs

$$
A_{Q}=\frac{I^{R}-I^{L}}{I^{R}+I^{L}}, \quad \Delta x_{i}=x_{i}^{R}-x_{i}^{L}
$$

where $I$ is the current, $x_{i}$ are various position measurements and $R(L)$ stand for right and left helicity.

The correction analysis is done by using five specific beam position monitors: 4ax, 4ay, 4bx, 4by and 12x. With these measurements one can determine the position, angle and energy of the beam at the target. Corrections to the raw asymmetry can be applied by using

$$
A_{\text {corr }}=A_{\text {raw }}-\Sigma_{i=1}^{5} \beta_{i} \Delta x_{i},
$$

where $A_{\text {raw }}=A_{\text {det }}-A_{Q}, \beta_{i}$ are the detector sensitivities to motion differences $\Delta x_{i}$ measured by each of the beam position monitors. Since the detector flux is normalized 

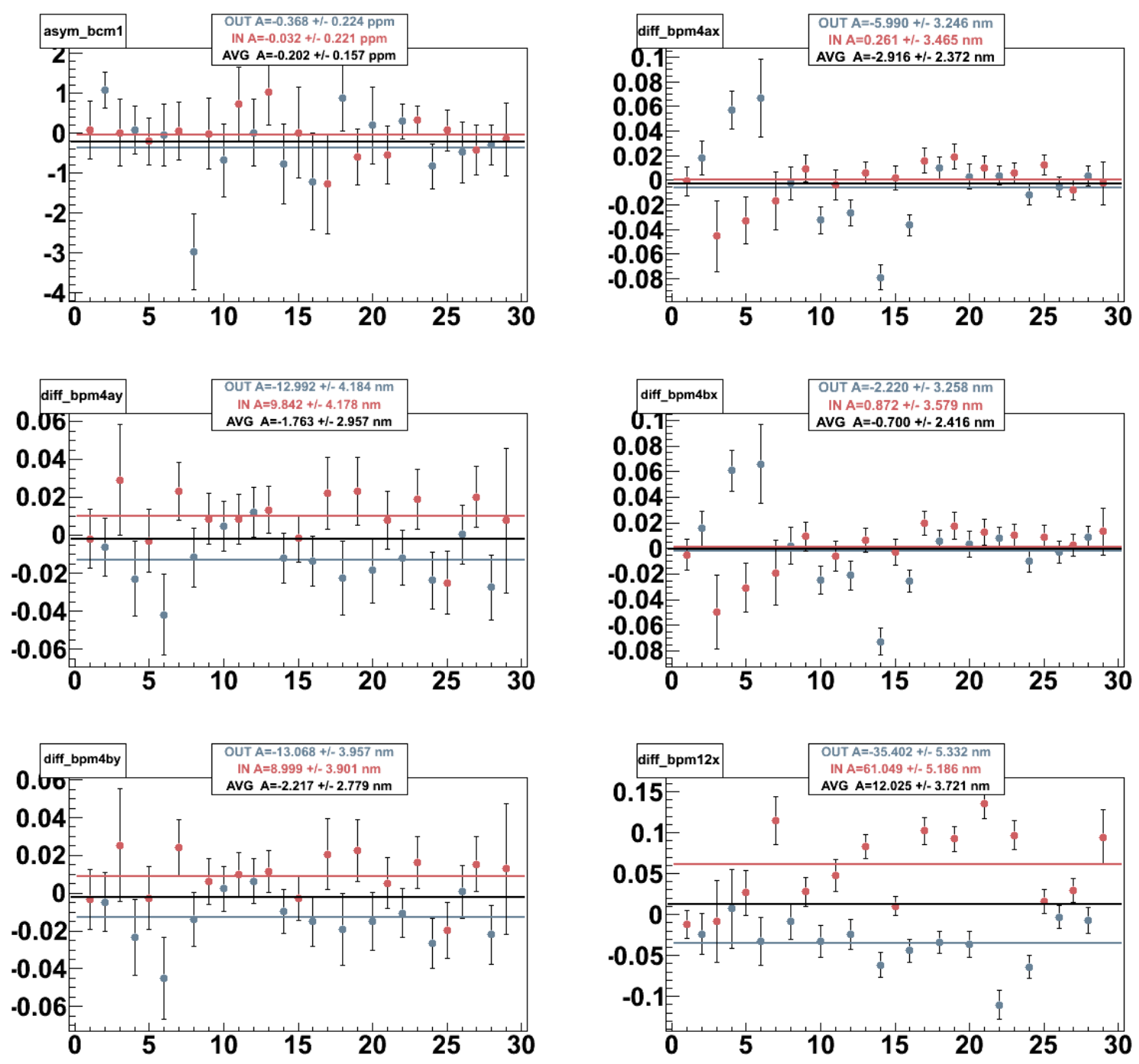

Figure 4.3: Summary plots for the main beam current and beam position monitors used in the final HAPPEx-III analysis. The data in blue was taken with IHWP OUT and the red data was taken with IHWP IN. Note that when averaging both sets of data, the position differences go to zero (black line). 


\begin{tabular}{|c|c|c|c|}
\hline & IHWP OUT & IHWP IN & IHWP BOTH \\
\hline bcm1 $(\mathrm{ppm})$ & $-0.368 \pm 0.224$ & $-0.032 \pm 0.221$ & $-0.202 \pm 0.157$ \\
\hline $\mathrm{bpm} 4 \mathrm{ax}(\mathrm{nm})$ & $-5.990 \pm 3.246$ & $0.261 \pm 3.465$ & $-2.916 \pm 2.372$ \\
\hline $\mathrm{bpm} 4 \mathrm{ay}(\mathrm{nm})$ & $-12.992 \pm 4.184$ & $9.842 \pm 4.178$ & $-1.763 \pm 2.957$ \\
\hline $\mathrm{bpm} 4 \mathrm{bx}(\mathrm{nm})$ & $-2.220 \pm 3.258$ & $0.872 \pm 3.579$ & $-0.700 \pm 2.416$ \\
\hline $\mathrm{bpm} 4 \mathrm{by}(\mathrm{nm})$ & $-13.068 \pm 3.957$ & $8.999 \pm 3.901$ & $-2.217 \pm 2.779$ \\
\hline $\mathrm{bpm} 12 \mathrm{x}(\mathrm{nm})$ & $-35.402 \pm 5.33$ & $61.049 \pm 5.186$ & $12.025 \pm 3.721$ \\
\hline
\end{tabular}

Table 4.2: Helicity Correlated charge asymmetry and position differences.

\begin{tabular}{|l|r|r|r|}
\hline & det 1 & $\operatorname{det} 2$ & det all \\
\hline bpm4ax & $-2.795 \pm 0.324$ & $0.438 \pm 0.326$ & $-1.135 \pm 0.234$ \\
\hline bpm4ay & $1.451 \pm 0.373$ & $1.266 \pm 0.376$ & $1.357 \pm 0.268$ \\
\hline bpm4bx & $-1.703 \pm 0.318$ & $1.471 \pm 0.319$ & $0.091 \pm 0.229$ \\
\hline bpm4by & $1.342 \pm 0.397$ & $0.100 \pm 0.398$ & $0.690 \pm 0.285$ \\
\hline bpm12x & $-0.590 \pm 0.064$ & $0.142 \pm 0.063$ & $-0.214 \pm 0.046$ \\
\hline
\end{tabular}

Table 4.3: Linear regression slopes

with respect to the measured beam intensity, no extra correction is needed to account for $A_{Q}$. Therefore, the charge asymmetry can only contribute a false asymmetry if there is a non-linearity in the beam current monitor or detector responses.

Ideally, the values of $\Delta x_{i}$ should be as close to zero as possible. The laser optics system that delivers the circularly polarized laser is designed to minimize such helicitycorrelated false asymmetries. Figure 4.3 shows the measurements made by the beam current monitor and all five beam position monitors listed above. The mean values for the entire data set, as well as for each IHWP state are listed in Table 4.2. These results show the usefulness of the passive helicity reversal provided by the IHWP. For example, while the $12 \mathrm{x}$ monitor measures a relatively large position difference for each IHWP state, when the entire data set is included, the effect becomes much smaller.

The detector sensitivities to each $x_{i}$ can be found by doing a linear regression analysis and are shown in Table 4.3. More accurate measurements of these sensitivities can be achieved by using beam modulation analysis. Both methods are used and the 

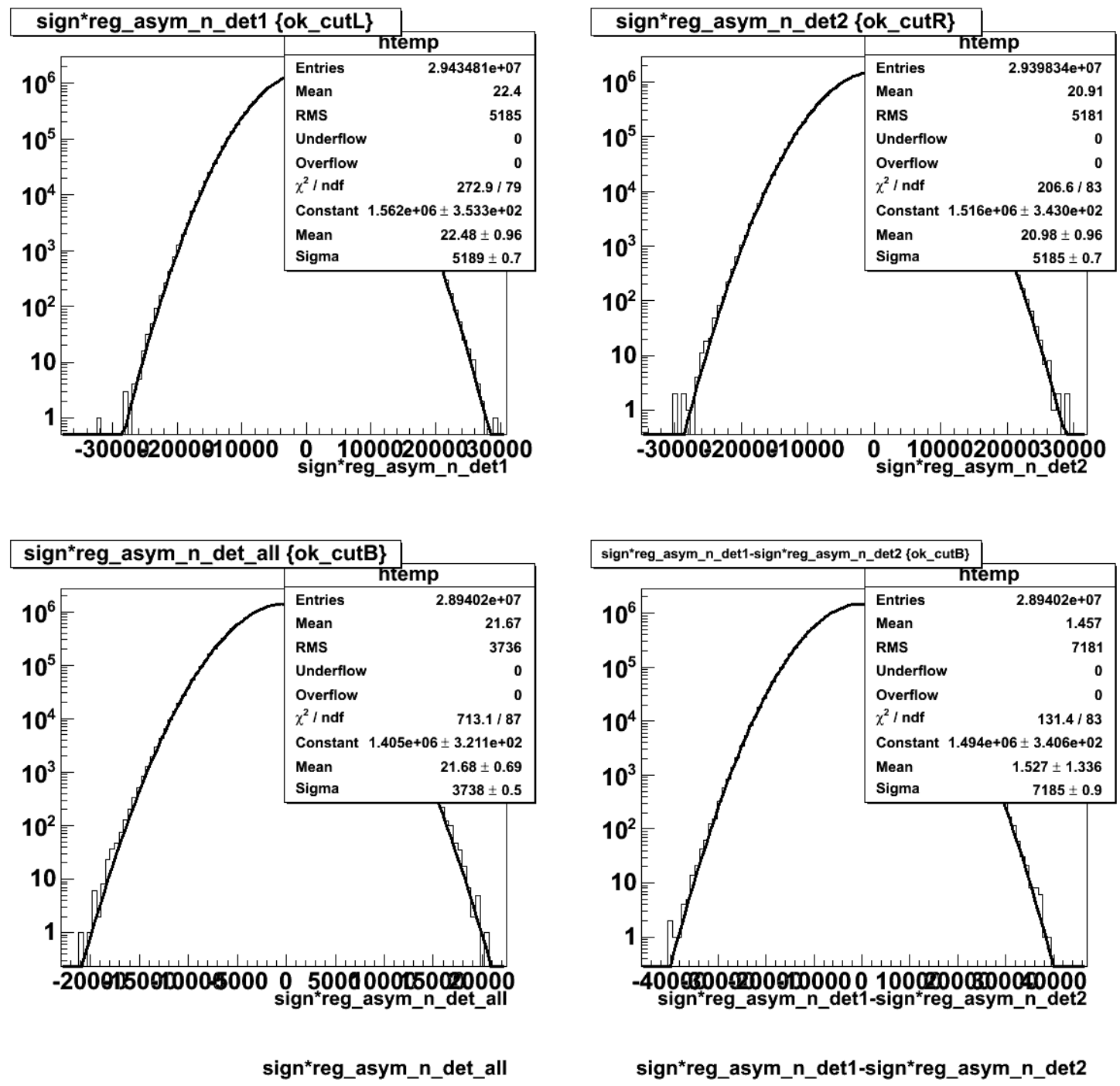

Figure 4.4: Sign-corrected regressed asymmetries, in units of parts-per-million, calculated for all data that passed all data quality cuts. 
results are compared. Ultimately, the values used are those obtained through the beam modulation method, also known as 'Dithering analysis'.

\subsubsection{Beam Modulation Analysis}

As was described in Section 2.4.3, there are several coils installed throughout the Hall A beamline used to introduce small variations in certain beam parameters. This technique is used to understand the cross-section sensitivity to small variations in beam position and energy. These sensitivities are then used to correct the raw asymmetry for any helicity-correlated false asymmetries

$$
A_{\text {corr }}=A_{\text {raw }}-\Delta A_{H C}
$$

defined as

$$
\Delta A_{H C}=\Sigma_{i=1}^{5}\left(\frac{\partial \sigma}{\partial M_{i}}\right) \Delta M_{i}
$$

where $\partial \sigma / \partial M_{i}$ is the slope that measures the sensitivity to the $i^{\text {th }}$ beam monitor and $\Delta M_{i}$ are the measured helicity-correlated beam monitor differences. By studying how each beam monitor is affected by each individual coil, defined as $\partial M_{i} / \partial C_{j}$ ( $j$ is the number of coils), the detector sensitivities are found by solving a matrix inversion problem

$$
\mathrm{S}=\mathrm{DM}^{-1}
$$

where the matrices are defined as

$$
\begin{aligned}
\mathbf{D} & =\Sigma_{j}\left(\frac{\partial \sigma}{\partial C_{j}} \frac{\partial M_{k}}{\partial C_{j}}\right) / \sigma^{2} \\
\mathbf{M} & =\Sigma_{j}\left(\frac{\partial M_{i}}{\partial C_{j}} \frac{\partial M_{k}}{\partial C_{j}}\right) / \sigma^{2} \\
\mathbf{S} & =\frac{\partial \sigma}{\partial M_{i}} .
\end{aligned}
$$

It is essential to note that in order for this problem to have a solution, the beam optics used to modulate the beam must be set so that $|\mathbf{M}| \neq 0$.

Figure 4.5 is an example of a beam modulation cycle, where red represents small shifts in the $\mathrm{x}$-direction, blue represents the $\mathrm{y}$-direction and magenta is a small energy shift. These cycles took place throughout most of the data taking process, but was not available for the first three slugs. Figure 4.6 shows how the beam position and slope 

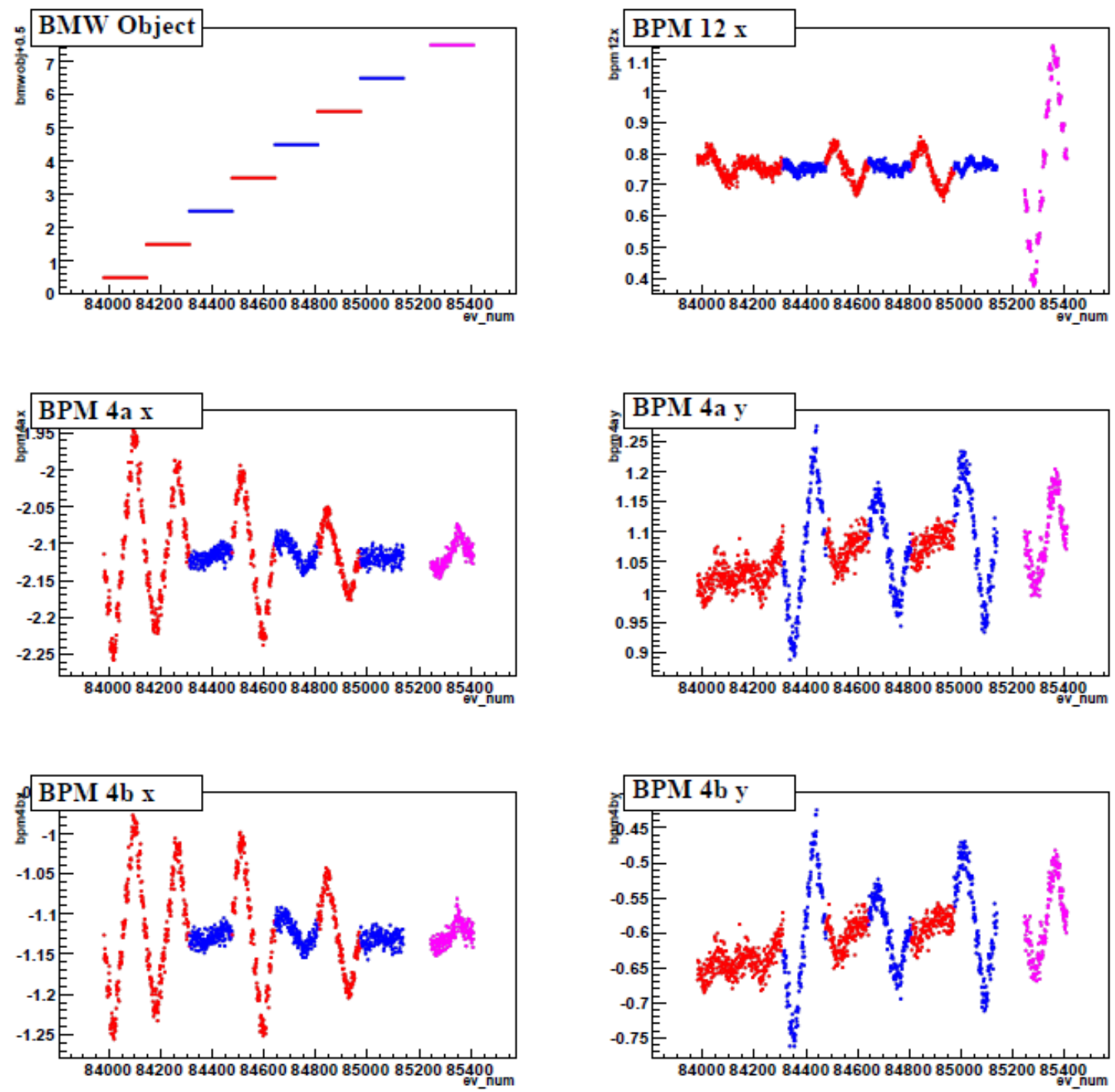

Figure 4.5: Sample plots from one dithering cycle. The red data represents kicks in the $\mathrm{x}$-direction, blue represents the $\mathrm{y}$-direction and magenta regresents small energy shifts. 

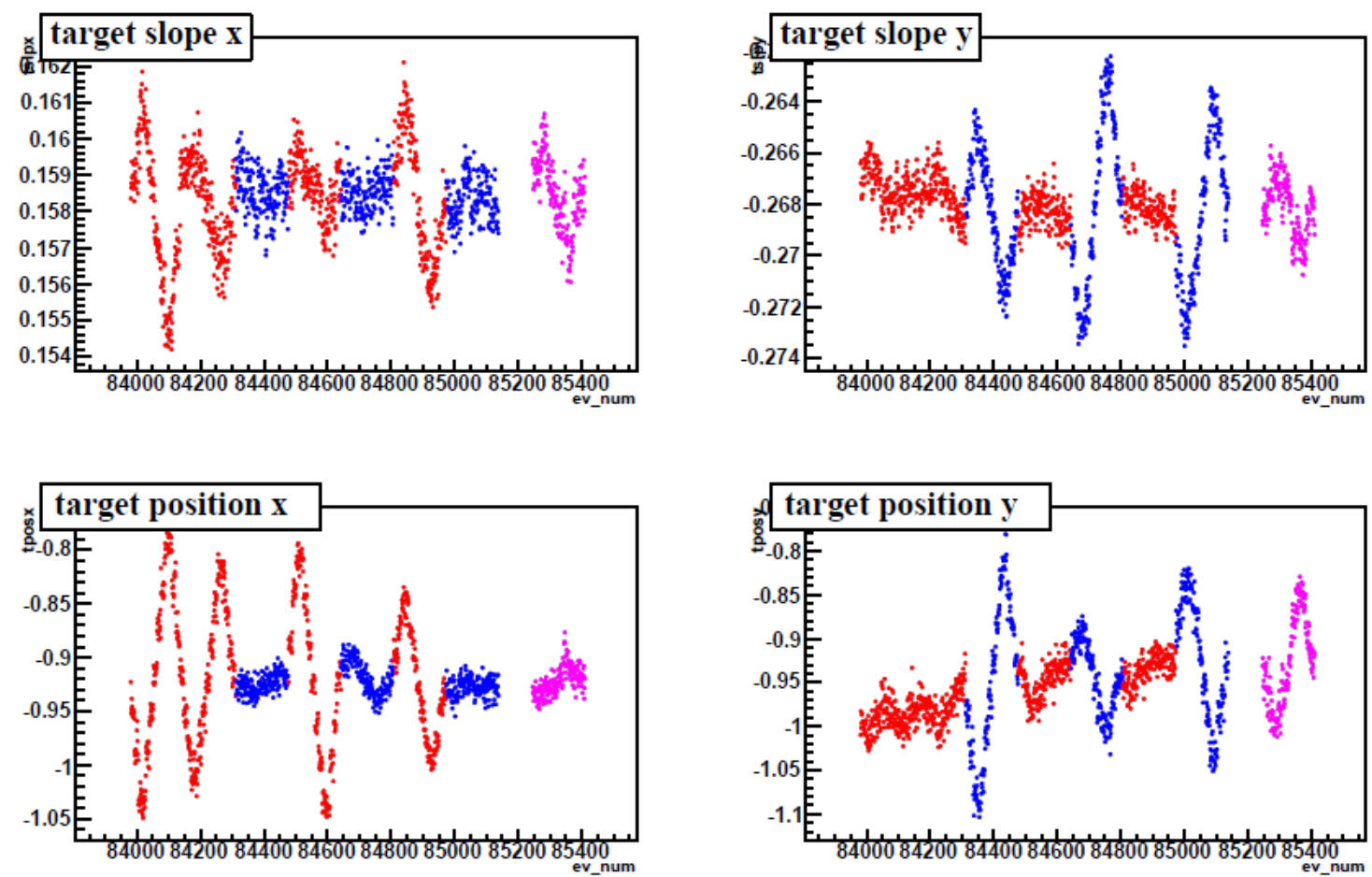

Figure 4.6: These plots show the effect of the modulation coils on the beam slope and position at the target.
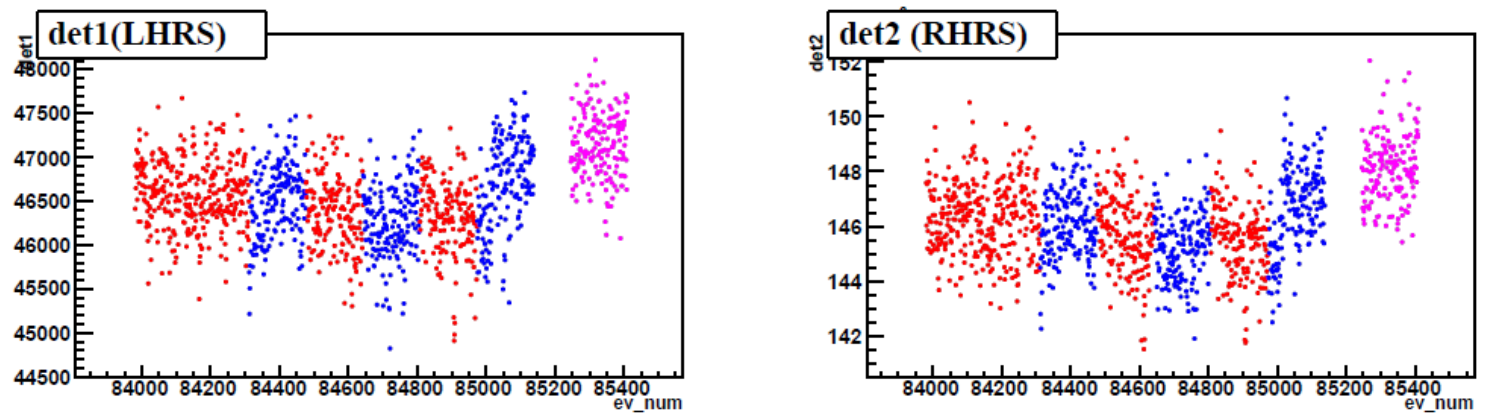

Figure 4.7: Detector signal during a dithering cycle. Note that there is no obvious effect on the detected flux, allowing for this data to be used as food production data. 


\begin{tabular}{|l|r|r|r|}
\hline & det 1 & det 2 & det all \\
\hline bpm4ax & $5.055 \pm 0.767$ & $-3.000 \pm 0.742$ & $0.991 \pm 0.688$ \\
\hline bpm4ay & $-2.678 \pm 1.266$ & $-1.316 \pm 1.214$ & $-1.918 \pm 1.134$ \\
\hline bpm4bx & $-10.047 \pm 0.836$ & $5.109 \pm 0.809$ & $-2.350 \pm 0.751$ \\
\hline bpm4by & $6.093 \pm 1.475$ & $2.969 \pm 1.418$ & $4.416 \pm 1.322$ \\
\hline bpm12x & $-0.255 \pm 0.065$ & $0.071 \pm 0.062$ & $-0.085 \pm 0.058$ \\
\hline
\end{tabular}

Table 4.4: Dithering slopes

get affected at the target by the same modulation cycle. While there is noticeable beam jitter at the target, Figure 4.7 shows that the effects on the detector signals is barely noticeable during the same period of time as the previous figures. In order to avoid degrading the main counting statistics of the detectors, ditherings slopes are averaged over many cycles so that we can understand the effects of these small fluctuations.

The sensitivity slopes, quoted in Table 4.4, were used to adjust the data set from slug 3 to slug 28. Slugs 0-2 were corrected using the regression slopes from Table 4.3. When averaged over the entire data set, the helicity-correlated contribution from the beam properties was about $0.9 \%$. This corresponds to $20 \mathrm{ppb}$ (parts per billion) in the intensity, $3 \mathrm{ppb}$ in energy and $3 \mathrm{~nm}$ in position. Figure 4.8 shows histograms of the entire data set after applying the appropriate regression and dithering slope corrections, summarized in Table 4.5. Note that the mean value of the det all asymmetry has been reduced by about 20 ppb from the mean value of the raw asymmetry in Figure 4.2.

\section{5 $Q^{2}$ Determination}

As part of finding an accurate value for the parity violating asymmetry, determining the value of the average four momentum transfer squared of the experiment is essential. The four momentum transfer of a scattered electron is defined as

$$
Q^{2}=-q^{2}=-\left(q_{i}-q_{f}\right)^{2}=2 E_{\text {beam }} E^{\prime}(1-\cos \Theta)
$$

where $q_{i}$ and $E$ are the four-momentum and energy of the incoming electron and $q_{f}, E^{\prime}$ and $\Theta$ are the four-momentum, energy and angle of the outgoing, scattered 

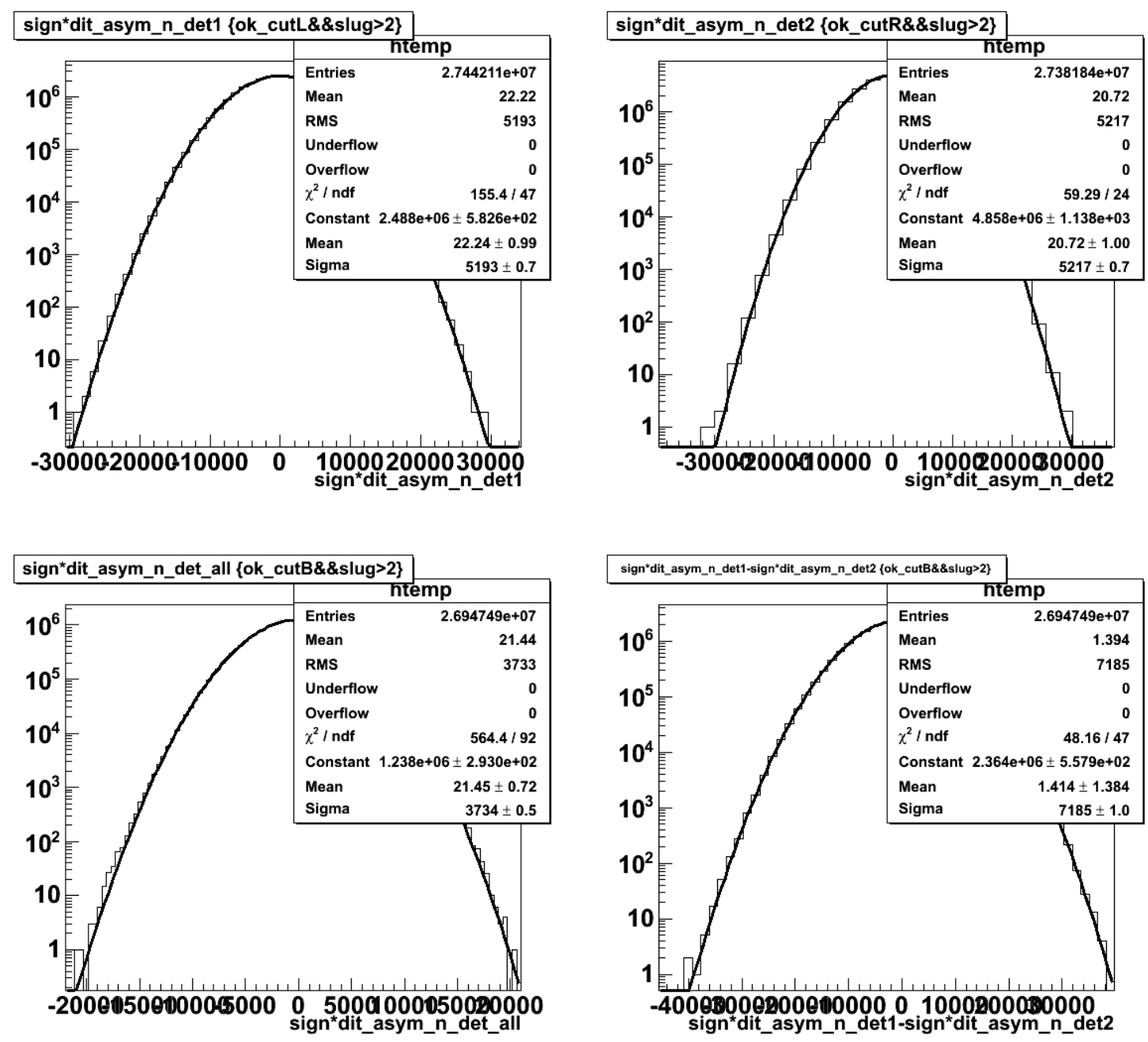

Figure 4.8: Sign-corrected, dither-adjusted asymmetries, in units of parts-permillion, calculated for all data that passed all data quality cuts. 


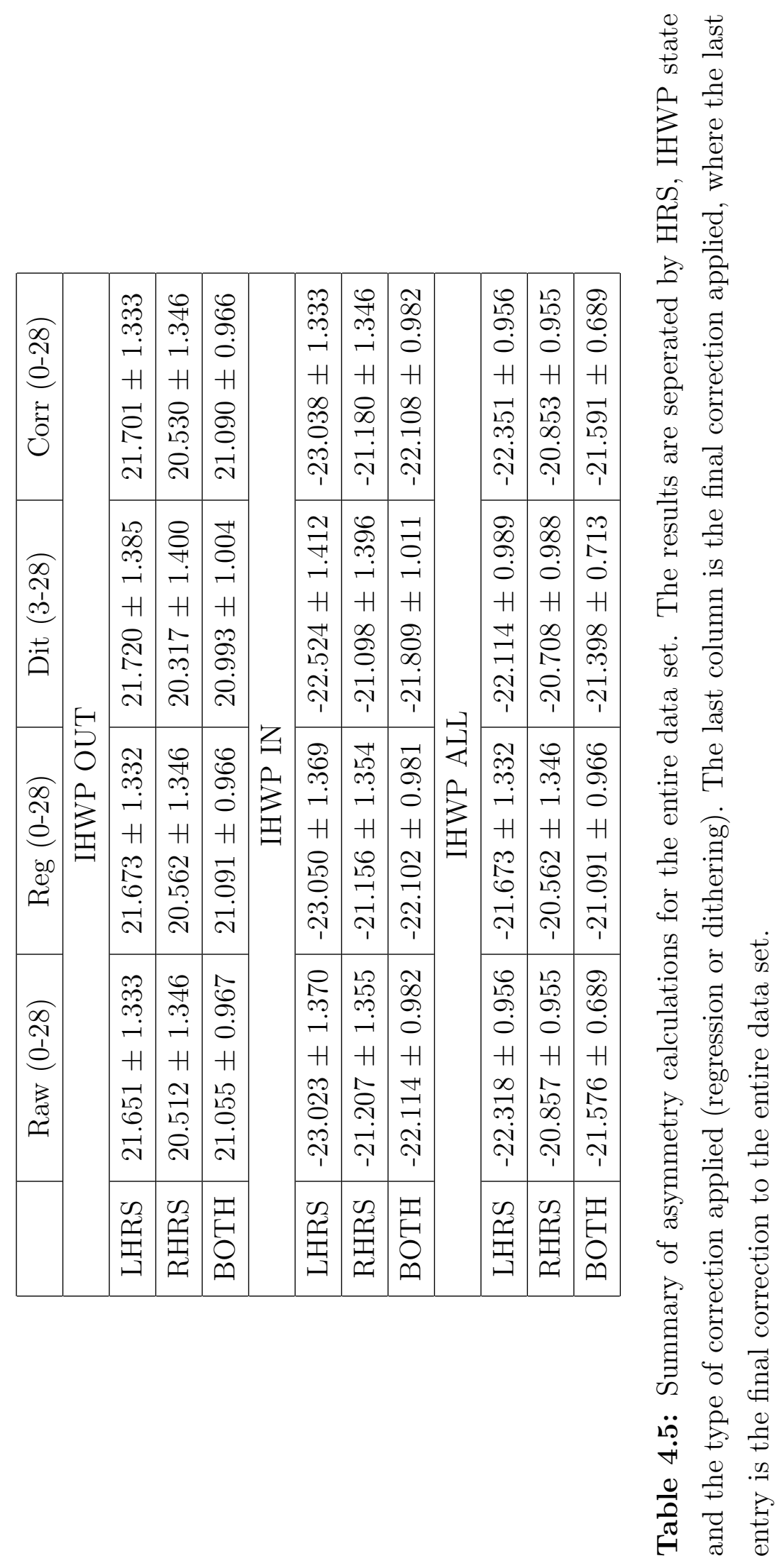



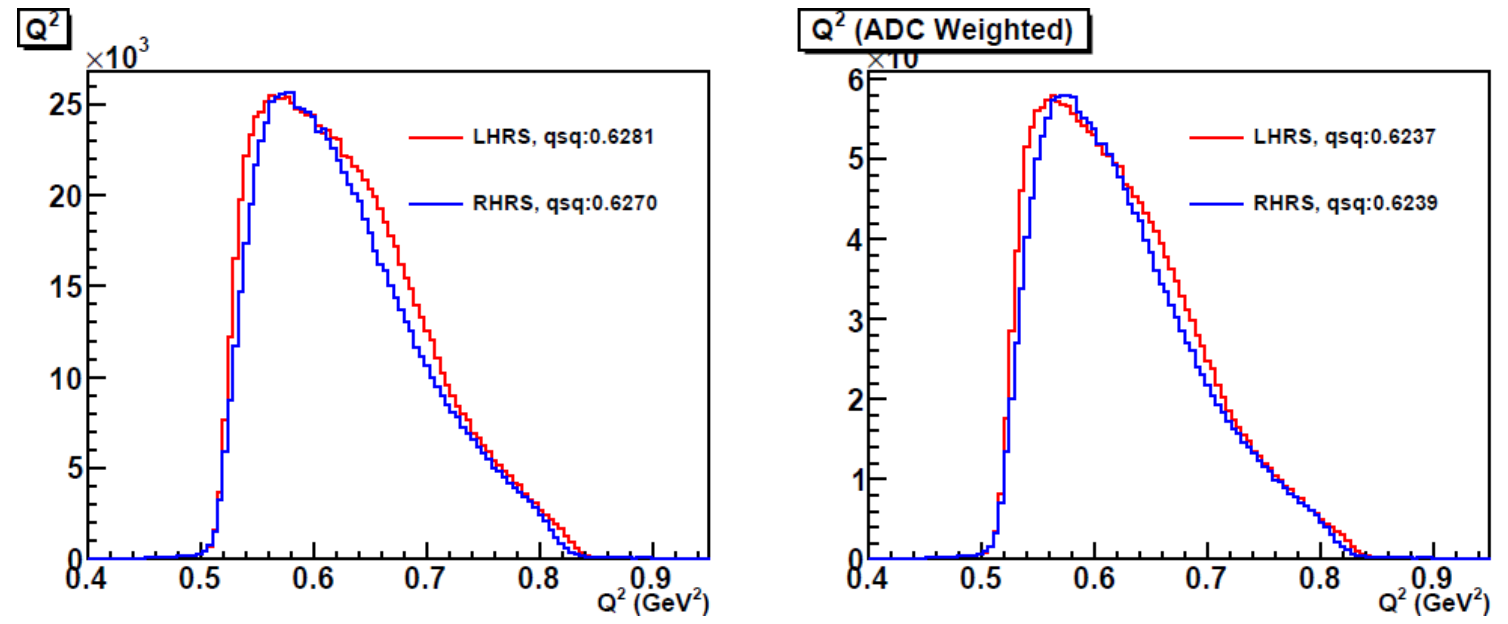

Figure 4.9: Both plots show $Q^{2}$ histograms for the Left (red) and Right (blue) HRS. The plot on the right shows the histogram after weighing, which corrects for the attenuation along the detector.

electron in the lab frame. The values of $E_{\text {beam }}, E^{\prime}$ and $\Theta$ can be measured independently to provide a redundant check of the four-momentum measurement. A detailed description of how $Q^{2}$ was determined can be found in [35]. Following is a summary of this process.

These measurements are made at low currents of about 2-5 $\mu \mathrm{A}$ every couple of weeks throughout the data taking process. For this, the standard Hall A detectors and counting DAQ are used along with the focal plane HAPPEx detectors. The VDCs are used to collect tracking information that helps us measure $\theta_{\text {det }}, \phi_{\text {det }}, x_{\text {det }}$ and $y_{\text {det }}$ at the detector, which are needed to find $\Theta$, the scattering angle in the xyz space

$$
\Theta=\cos ^{-1}\left(\frac{\cos \Theta_{0}-\phi \sin \Theta_{0}}{\sqrt{1+\theta^{2}+\phi^{2}}}\right),
$$

where $\Theta_{0}$ is the central angle of the HRS, as well as $\theta$ and $\phi$, the incoming angle at the focal plane.

The value of $E_{\text {beam }}$ used was $3.482 \mathrm{GeV}$ and it accounts for ionization energy losses inside the LH2 target. On average, the total ionization loss due to multiple scattering in a $25 \mathrm{~cm}$ long LH2 target is about $4 \mathrm{MeV}$. While the true energy of the beam is $3.484 \mathrm{GeV}$, an average ionization loss of $2 \mathrm{MeV}$ was used assuming that most of the elastic scattering occurs at the center of the target. The scattered energy $E^{\prime}$ can then 


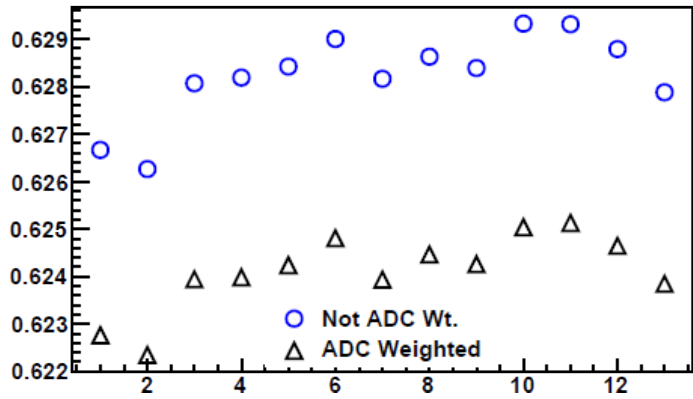

\section{Qsq RHRS}

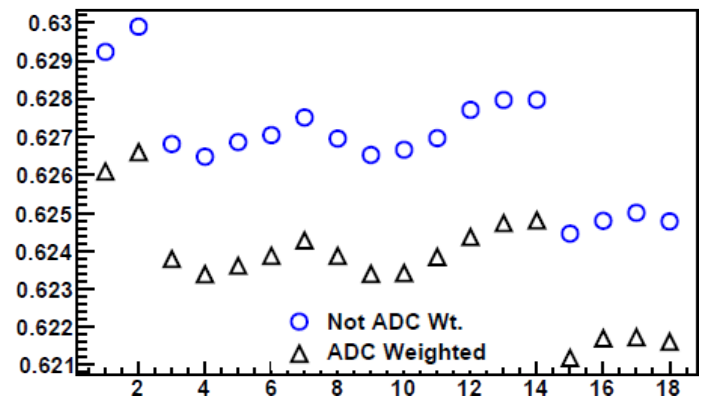

Figure 4.10: Average weighted (black triangles) and unweighted (blue circles) $Q^{2}$ values for at data taken throughout the experiment. The variations seen can be explained by differences in the beam position.

be found by using

$$
E^{\prime}=\frac{E_{\text {beam }}}{1+\frac{E_{\text {beam }}}{m_{H}}(1-\cos \Theta)},
$$

where $m_{H}=0.938 \mathrm{GeV} / \mathrm{c}^{2}$ is the proton mass.

To obtain an accurate final $Q^{2}$ calculation, they must be energy weighted to account for signal attenuation along the detectors. This is due to particles of the same energy hitting the detector at differing distances from the PMT cathode. Figure 4.9 shows sample distributions of our $Q^{2}$ calculations before and after weighting. While the distributions from each HRS are slightly different, these effects can be accounted for by considering variations in the HRS angles and the HRS acceptances.

Figure 4.10 shows all the results for $Q^{2}$ determinations throughout the HAPPExIII experiment. The full RHRS data shows fluctuations over time that can be grouped into three main sections. It was found that these variations are caused by changes in beam position at the target in the x-direction. A summary of all the $Q^{2}$ weighed averages are listed in Table 4.6 for each HRS. Final charge weighted averages are used to find a final value of $Q^{2}=0.6241 \pm 0.0032 \mathrm{GeV}^{2}$.

\subsection{Backgrounds}

When taking data using our integrating DAQ, there is no way of separating background events from those that contribute to our main elastic signal. For this reason, we must correct the value of our raw asymmetry by doing dedicated studies of such 


\begin{tabular}{|c|c|c|c|c|c|}
\hline & & & \multicolumn{3}{|c|}{ LHRS } \\
\hline Date & bpm4ax & bpm4bx & $Q^{2}$ & $Q_{w t}^{2}$ & Charge (mC) \\
\hline Sep01-Sep04 & -0.13 & -2.23 & 0.6264 & 0.6222 & 2744.48 \\
\hline Sep04-Sep24 & -0.30 & -0.80 & 0.6284 & 0.6241 & 37649.30 \\
\hline Sep24-Oct20 & -0.31 & -1.30 & 0.6281 & 0.6238 & 56048.92 \\
\hline & & & \multicolumn{3}{|c|}{ RHRS } \\
\hline Sep01-Sep04 & -0.13 & -2.23 & 0.6291 & 0.6260 & 2744.48 \\
\hline Sep04-Sep24 & -0.30 & -0.80 & 0.6272 & 0.6241 & 37649.30 \\
\hline Sep24-Oct20 & -0.31 & -1.30 & 0.6277 & 0.6245 & 56048.92 \\
\hline Oct24-Oct25 & -0.54 & -1.32 & 0.6249 & 0.6218 & 2281.79 \\
\hline
\end{tabular}

Table 4.6: Summary of average values of $Q^{2}$ over several periods of time were the beam position was slightly different. Note that there is more data from the Right HRS because during that time, one of the sprecrometer magnets was malfunctioning.

backgrounds. There were several types of backgrounds that we studied and corrected for. The main two sources of background are believed to be from inelastically scattered electrons being re-scattered off the spectrometer walls, and from scattering off the target's aluminum end caps. Following is a detailed discussion of these corrections and the systematic errors attributed to them.

\subsubsection{Inelastic Background}

The High Resolution Spectrometers used in Hall A are very effective at separating inelastic backgrounds from the elastic signal we seek. Regardless, it is possible for some of these inelastically scattered electrons to reach our detectors by rescattering off the spectrometer walls. This rescattered signal can be expressed as an integral over the scattered energies

$$
B=\int_{E_{t h r}}^{E_{\max }} d E P_{r s}(E) \times R(E)
$$

where $E_{t h r}$ is the inelastic threshold and $E_{\max }$ is the maximum energy loss. The background depends on $R(E)$, the ratio of inelastic to elastic cross sections,

$$
R(E)=\left(\frac{d \sigma}{d \Omega d E}\right)_{\text {inel }} /\left(\frac{d \sigma}{d \Omega}\right)_{\text {elastic }}
$$




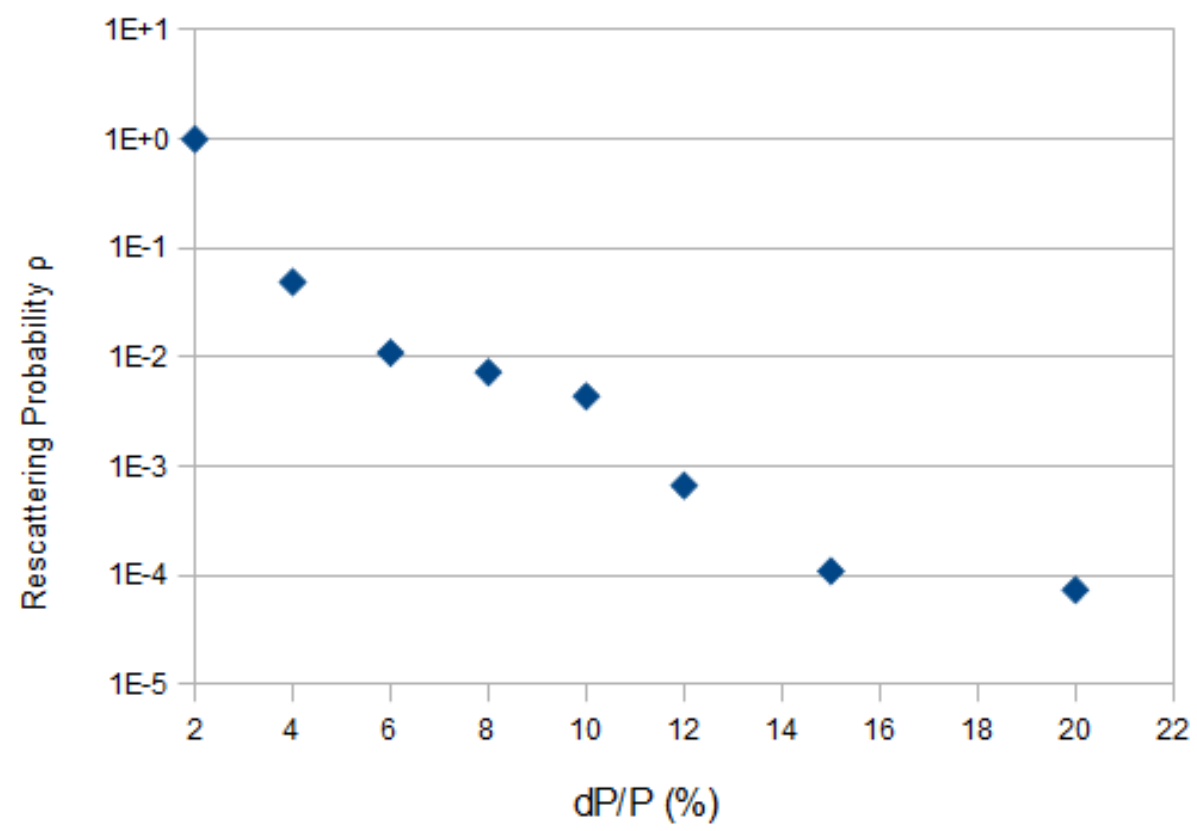

Figure 4.11: Measurements of the probability for a particle to re-scatter into the detector with respect to the percentage deviation from the nominal beam momentum.

and the rescattering function $P_{r s}$,

$$
P_{r s}=\rho \times\left(\frac{E_{d e p}}{E_{0}}\right)
$$

where $\rho$ is the rescattering probability, $E_{d e p}$ is the energy deposited and $E_{0}$ is the energy of the elastically scattered electrons. The rescattering function was measured by varying the strength of the dipole field in the spectrometers (see Fig. 4.11).

In doing so, the elastically scattered electrons are forced to follow the trajectories of the inelastic electrons and the probability of these particles to hit our detectors is measured using the spectrometer counting DAQ and the HAPPEx integrating DAQ. Since we only have discrete values for the rescattering function, dependent on the dipole settings used to bend the particle's trajectories, we use linear interpolation between each data value to increase the accuracy of the background calculation.

To obtain values for $R(E)$, the inelastic cross-sections were calculated using a parametrization of SLAC data [31]. Even though this model was used for experiments such as HAPPEx we want to cross check it with an empirical fit of inclusive electronproton cross sections done by Hall $\mathrm{C}$ at Jefferson Labs [32]. This fit covers a wider 


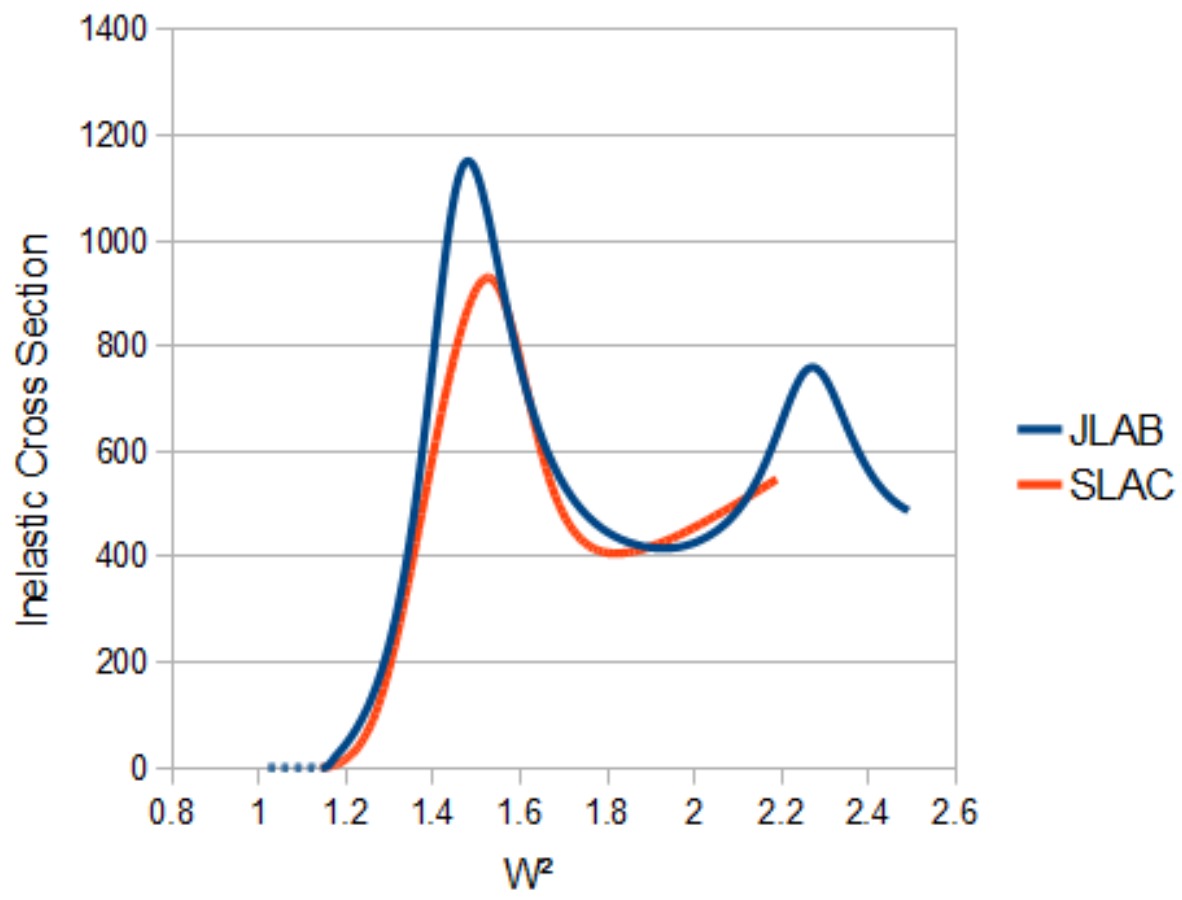

Figure 4.12: Energy Spectrum at HAPPEx III kinematics. (Red line - SLAC Model, Blue line - Hall C Model)

kinematic range and is believed to work well from very high momentum transfer values down to $Q^{2} \approx 0 \mathrm{GeV}^{2}$. Figure 4.12 shows the inelastic cross-sections at HAPPEx III kinematics calculated by using both methods, where $W$ is the invariant mass. The SLAC data clearly shows smaller cross-sections for the kinematic range we are interested in.

Using Equation 4.16 we find that the background from inelastic electrons rescattering from the spectrometer is $B=(0.32 \pm 0.05) \%$ of the total signal detected. This background is mainly due to the first peak in Figure 4.12, caused by the $\Delta$ resonance. To be able to correct our data, we must use the known parity-violating asymmetry due to the $\Delta$ resonance [36],

$$
A_{\Delta}^{P V} \approx \frac{-G_{F}\left|Q^{2}\right|}{2 \sqrt{2} \pi \alpha}\left(1-2 \sin ^{2} \theta_{W}\right)
$$

The asymmetry is calculated to be $A_{\Delta}^{P V} \approx 63 \mathrm{ppm}$ at our $Q^{2}$ with an uncertainty of $20 \%$. 


\subsubsection{Scattering off target end caps}

The liquid hydrogen target is housed inside an aluminum cell, described in Section 2.5. For this reason, corrections must be made to the integrated detector signal to account for backgrounds from electrons that interact with the aluminum. To do this, aluminum foils are used to simulate the width and separation of the front and back end caps of the cell. Measurements were also conducted using the evacuated target cell. Dedicated studies are conducted at low beam currents to measure the contribution from this background. The fraction of this background was found to be $(1.15 \pm$ $0.35) \%$. The asymmetry was estimated to be $-34.5 \mathrm{ppm}$ with an uncertainty of $30 \%$ [37].

\subsection{Finite Acceptance}

When the value of $Q^{2}$ is measured, the signal is affected by radiative losses in the target as well as the finite acceptance of the HRS spectrometer. This makes it so that the elastic peak at the detectors comes from a range of $Q^{2}$ values. For an accurate determination of $A_{P V}$, corrections must be made to get a single $Q^{2}$ value from the measured range. This is done by calculating a kinematic acceptance factor $K$ by using a simulation,

$$
K=\frac{A\left(\left\langle Q_{\text {det }}^{2}\right\rangle\right)}{\left\langle A\left(Q_{\text {vert }}^{2}\right)\right\rangle}
$$

where $A\left(\left\langle Q_{\text {det }}^{2}\right\rangle\right)$ is the asymmetry of the $Q^{2}$ measured after going throught the HRS and $\left\langle A\left(Q_{v e r t}^{2}\right)\right\rangle$ is the asymmetry of the scattering vertex.

The simulation package used, the Hall A Monte Carlo (HAMC), calculates how elastically scattered electrons are created in e-p scattering, giving rise to $\left\langle A\left(Q_{v e r t}^{2}\right)\right\rangle$. After that, it transports the electrons through the HRS magnets all the way to the detector focal plane, providing $A\left(\left\langle Q_{\text {det }}^{2}\right\rangle\right)$. Figure 4.13 shows the results of these simulations for both HRS. The plots also show how these values would be affected by a small variation $( \pm 10 \mathrm{~mm})$ in the acceptance collimator dimensions, which are $121.8 \times 62.9 \mathrm{~mm}$. The effect on the value of $K$ is very small, as seen in Table 4.7 Considering these effects, we assign a value of $K=0.995 \pm 0.002$ to the kinematic acceptance factor. 

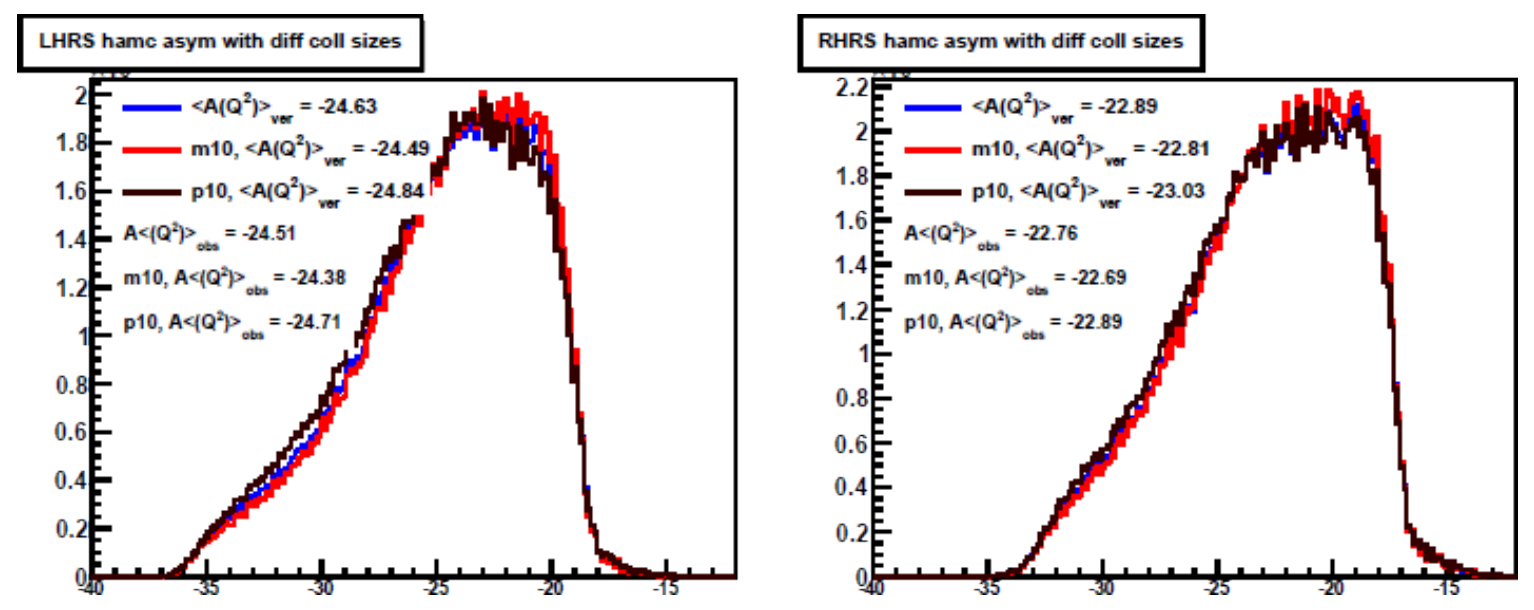

Figure 4.13: Both plots show simulation results for the vertex asymmetries for both HRS. They also show the results when varying the spectrometer acceptance by \pm 10 $\mathrm{mm}$ in both the $\mathrm{x}$ and $\mathrm{y}$ directions. These values are conpared with the asymmetries of the observed $Q^{2}$ averages.

\begin{tabular}{|c|c|c|c|c|c|c|}
\hline & \multicolumn{3}{|c|}{ LHRS } & \multicolumn{3}{c|}{ RHRS } \\
\hline & coll nom & coll m10 & coll p10 & coll nom & coll m10 & coll p10 \\
\hline$\left\langle A\left(Q_{\text {vert }}^{2}\right)\right\rangle$ & -24.63 & -24.49 & -24.84 & -22.89 & -22.81 & -23.03 \\
\hline$A\left(\left\langle Q_{\text {det }}^{2}\right\rangle\right)$ & -24.51 & -24.38 & -24.71 & -22.76 & -22.69 & -22.89 \\
\hline$K$ & 0.995 & 0.996 & 0.995 & 0.994 & 0.995 & 0.994 \\
\hline
\end{tabular}

Table 4.7: Summary of HAMC simulation results for the acceptance factor $K$. The effects of small variations $( \pm 10 \mathrm{~mm}$ in both $\mathrm{x}$ and $\mathrm{y})$ in the dimensions of the collimator acceptance are also listed and are negligible. 


\begin{tabular}{|c|c|c|}
\hline Data Set & Polarization (\%) & Error (\%) \\
\hline $\mathrm{C} 1$ & 89.11 & 0.19 \\
\hline $\mathrm{C} 2$ & 89.74 & 0.08 \\
\hline $\mathrm{C} 3$ & 90.13 & 0.11 \\
\hline $\mathrm{C} 4$ & 89.36 & 0.10 \\
\hline \hline $\mathrm{C}_{\text {tot }}$ & 89.41 & 0.861 \\
\hline $\mathrm{M}_{\text {tot }}$ & 89.22 & 1.517 \\
\hline All Data & 89.36 & 0.747 \\
\hline
\end{tabular}

Table 4.8: All beam polarization measurements are summarized along with statistical errors of the partial Compton data. The errors quoted for the full data sets include both statistical and systematic contributions.

\subsection{Beam Polarization}

Throughout data taking the experiment, the beam polarization was measured using two methods described in Chapter 2. The Moller Polarimeter was used to make 7 independent measurements, while the Compton Polarimeter made measurements during the entire time that production data was taken. The polarization value must be used to normalize the asymmetry measurement, and is the biggest correction made to find the final physics asymmetry.

During the HAPPEx-III run, there were several time periods in which the measured polarization was affected by slightly different laser tunes. These measurements are shown in Figure 4.14, where different color data points represent the different laser tunes. The regions of non-uniform polarization can be aproximated with straight lines. The mean values for the Compton measurements for the four different segments of data are summarized in Table 4.8. From this data we obtain a very accurate final value for the degree of beam polarization of $P_{b}=89.36 \pm 0.747 \%$. At the time of this measurement, it was the first ever result with such a high degree of precision. 


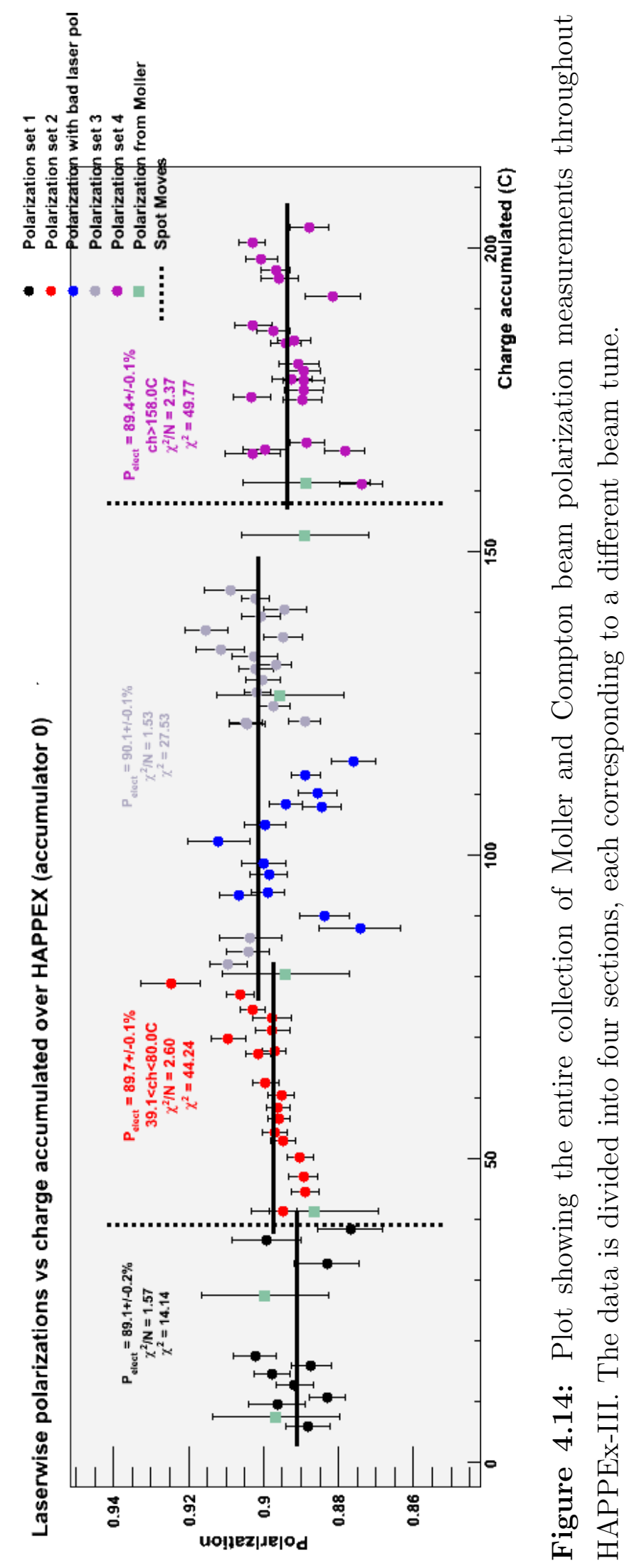




\begin{tabular}{|c|c|c|}
\hline Term & Value & Error $(\%)$ \\
\hline$K$ & 0.995 & 0.002 \\
\hline$P_{b}$ & 0.894 & 0.75 \\
\hline$f_{\text {alum }}$ & $1.15 \%$ & 0.35 \\
\hline$A_{\text {alum }}$ & $-34.5 \mathrm{ppm}$ & 30 \\
\hline$f_{\text {res }}$ & $0.29 \%$ & 0.08 \\
\hline$A_{\text {res }}$ & $-63 \mathrm{ppm}$ & 20 \\
\hline
\end{tabular}

Table 4.9: Summary of all the values used to calculate the final physics asymmetry, with their systematic errors.

\begin{tabular}{|l|r|r|}
\hline Source & Value(\%) & Error (\%) \\
\hline Detector Linearity & 0.0 & 0.5 \\
\hline Beam Asymmetries & -0.9 & 0.2 \\
\hline Backgrounds & -1.0 & 0.8 \\
\hline Acceptance & -0.5 & 0.2 \\
\hline Beam Polarization & 10.9 & 0.8 \\
\hline$Q^{2}$ & $\mathrm{n} / \mathrm{a}$ & 0.8 \\
\hline Total & 8.5 & 1.5 \\
\hline
\end{tabular}

Table 4.10: Summary of all contributions to the systematic error of $A_{P V}$. 


\subsection{Final Physics Asymmetry}

Equipped with the results of all the previous sections, the final parity-violating physics asymmetry can be found by using the expression introduced at the beginning of Chapter 2,

$$
A_{\text {phys }}=\frac{K}{P_{b}} \frac{A_{\text {corr }}-P_{b} \Sigma_{i} f_{i} A_{i}}{1-\Sigma_{i} f_{i}} .
$$

In this equation, $K$ is the acceptance factor, $L$ is linearity coefficient, $P_{b}$ is the degree of beam polarization, $f_{i}$ are the background fractions and $A_{i}$ are the asymmetries of the background processes.

All the values used to solve this equation are summarized in Table 4.9 along with their appropriate errors. Table 4.10 shows all the sources of systematic error for the final value. The final value obtained for $A_{P V}$ at $Q^{2}=0.624 \mathrm{GeV}^{2}$ was

$$
A_{P V}=-23.803 \pm 0.778(\text { stat }) \pm 0.359(\text { syst }) \text { ppm }
$$

where the first error represents a $3.28 \%$ measurement of the asymmetry. Systematic errors only add $1.5 \%$, making this experimental result statistics dominated. 


\section{CHAPTER 5}

\section{RESULTS AND CONCLUSIONS}

\subsection{Overview}

This thesis has discussed in detail work related to two parity-violating electronscattering experiments conducted at Jefferson Labs. The collaboration that worked on these experiments successfully developed new technologies to accomplish new measurements with unprecedented accuracy. These achievements will make it possible for future experiments to take place that will keep providing further knowledge about nucleon structure, neutron skin and physics beyond the Standard Model.

As for the HAPPEx-III experiment, let us now discuss how to find the strange quark contribution to the nucleon form factors from the physics asymmetry obtained at the end of Chapter 4. The implications of the new constraints set for the strange form factor contribution of all the HAPPEx measurements will be summarized.

\subsection{Determining Strange Quark Contribution}

The final physics asymmetry will now be used to find the linear combination of strange form factors $G_{E}^{s}+\eta G_{M}^{s}$ by using Equation 1.30. In this section, I will rearrange the equation in order to isolate the strange form factor contribution to the asymmetry

$$
\begin{array}{r}
A_{P V}^{e p}=\left[\frac{-G_{F} Q^{2}}{4 \pi \alpha \sqrt{2}}\right]\left\{\left(1-4 \sin ^{2} \theta_{W}\right)-\frac{\epsilon G_{E}^{p} G_{E}^{n}+\tau G_{M}^{p} G_{M}^{n}}{\epsilon\left(G_{E}^{p}\right)^{2}+\tau\left(G_{M}^{p}\right)^{2}}\right. \\
\left.-\frac{\epsilon G_{E}^{p} G_{E}^{s}+\tau G_{M}^{p} G_{M}^{s}}{\epsilon\left(G_{E}^{p}\right)^{2}+\tau\left(G_{M}^{p}\right)^{2}}-A_{A}\right\},
\end{array}
$$

where $\alpha$ is the fine structure constant, $\epsilon=\left[1+2(1+\tau) \tan ^{2}(\theta / 2)\right]^{-1}, \tau=Q^{2} /\left(4 M_{p}^{2}\right)$, $G_{F}$ is the Fermi constant and $A_{A}$ is the axial contribution to the asymmetry. Now the theoretical expectation for $A_{P V}$ becomes: $A_{P V}=A_{V}+A_{S}+A_{A}$ and the third term 


\begin{tabular}{|c|c|c|}
\hline Form factor & Value & Error \\
\hline$G_{E}^{p}$ & 0.279 & 0.005 \\
\hline$G_{M}^{p}$ & 0.789 & 0.009 \\
\hline$G_{E}^{n}$ & 0.049 & 0.006 \\
\hline$G_{M}^{n}$ & -0.555 & 0.002 \\
\hline
\end{tabular}

Table 5.1: Values used for the proton and neutron electric and magnetic form factors.

\begin{tabular}{|c|c|}
\hline Kinematic Constant & Measured Value \\
\hline$Q^{2}$ & $0.6241 \mathrm{GeV}^{2}$ \\
\hline$\theta_{l a b}$ & $13.7053^{\circ}$ \\
\hline$\tau$ & 0.1772 \\
\hline$\epsilon$ & 0.9671 \\
\hline
\end{tabular}

Table 5.2: Values used for kinematic constants determined during the experiment.

in Equation 5.1 can be referred to as $A_{S}$. This term depends only on the proton form factors and the kinematic factors $\epsilon$ and $\tau$, which give rise to $\eta=\tau G_{M}^{p} /\left(\epsilon G_{E}^{p}\right)=0.517$.

The values for the proton and neutron electromagnetic form factors used are summarized in Table 5.1, along with their corresponding errors. These were obtained by using a parametrization fit of form factor world data that includes the effects of two-photon exchange corrections [38]. The rest of the values needed to evaluate Equation 5.1 are listed in Table 5.2.

If strange quarks did not contribute to the vector form factors, the asymmetry for the $Q^{2}$ of this experiment would be expected to be $A_{N S}=A_{V}+A_{A}=-24.062 \pm 0.734$ ppm, where the main source of error is from uncertainties in the values of the nucleon electromagnetic form factors. By comparing the physics asymmetry, found to be $A_{P V}$ $=23.803 \pm 0.778$ (stat) \pm 0.359 (syst) ppm, with this value of $A_{N S}$, the strange quark contribution to the proton form factors is

$$
G_{E}^{s}+0.517 G_{M}^{s}=0.003 \pm 0.010 \text { (stat) } \pm 0.004 \text { (syst) } \pm 0.009(\mathrm{FF})
$$

Before going into a discussion about the implications of this measurement, we will first discuss past measurements of the strange vector form factors at several values of four-momentum transfer, most of which are displayed in Figure 5.1. Measurements 


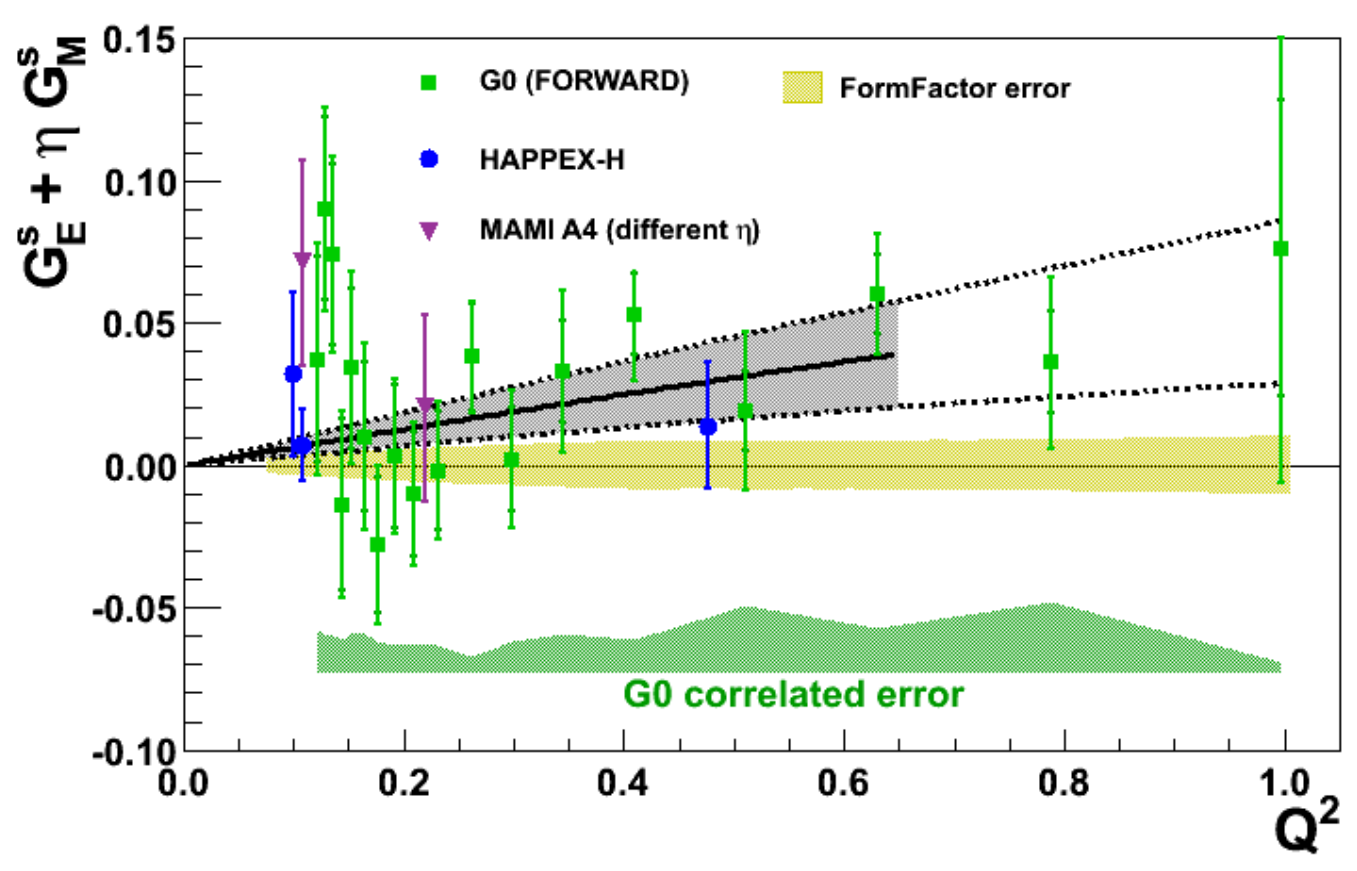

Figure 5.1: Results from A4, G0 and HAPPEx-H for strange quark vector formfactors. The green and yellow shaded regions represent G0 correlated error and form factor errors.

at the highest $Q^{2}$ values show a growing positive trend for the linear combination $G_{E}^{s}+\eta G_{M}^{s}$.

\subsection{Summary of Past Results}

In the past two decades, there have been numerous parity-violation experiments conducted to measure the strange quark contribution to the nucleon form factors. These measurements have been conducted at a wide range of momentum transfer squared, using different kinematics and targets to isolate the values of $G_{E}^{s}$ and $G_{M}^{s}$.

\subsubsection{SAMPLE}

The SAMPLE experiment was conducted in 1998-1999 at the MIT-Bates Laboratory and focused on finding the contribution of strange quarks to the proton's magnetic form factor by measuring a parity-violating asymmetry. It was designed to detect 


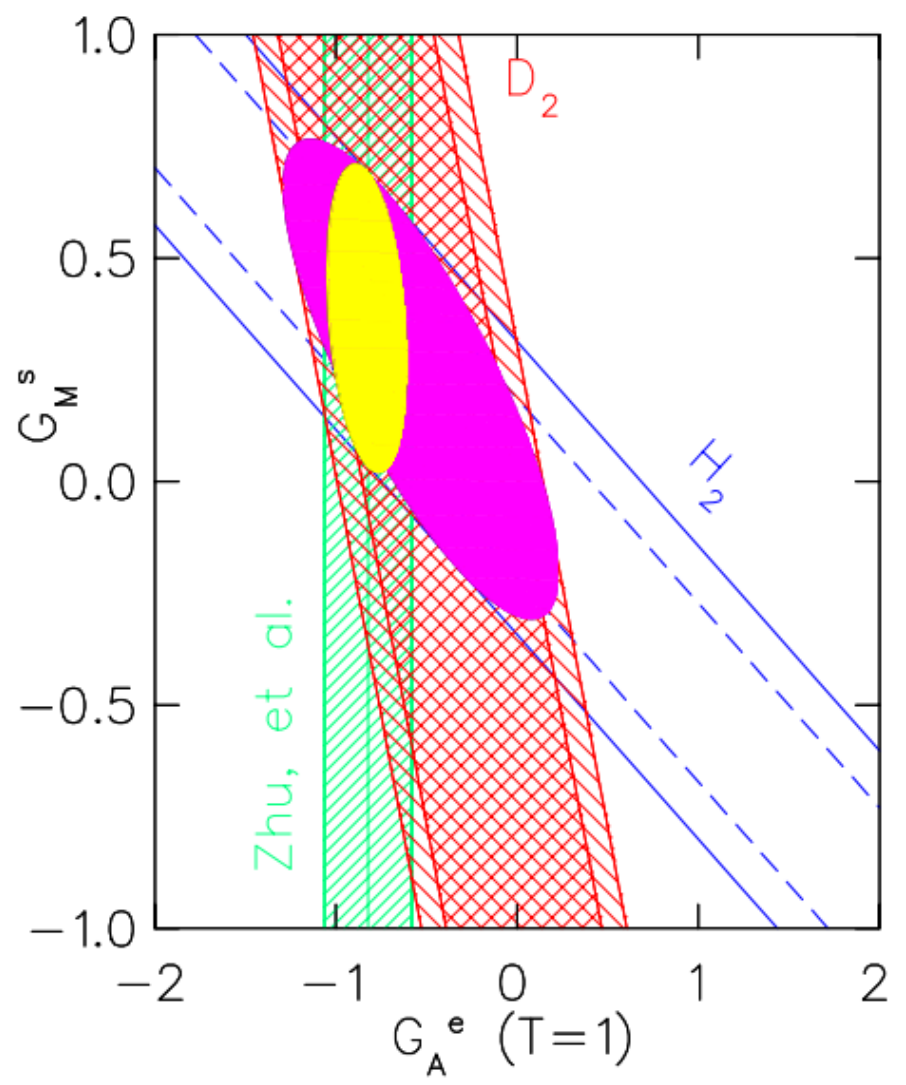

Figure 5.2: Results from the SAMPLE hydrogen (blue lines) and deuteron (red lines) data. Also shown is the theoretical expected value for $G_{A}^{e(T=1)}$ (green lines)

electrons scattered off a $40 \mathrm{~cm}$ liquid hydrogen target at backward angles and a low momentum transfer of $Q^{2}=0.1 \mathrm{GeV}^{2}$. The range of scattering angle measured was 138 to 160 and helps explore the kinematic range were the asymmetry is sensitive to $G_{M}^{s}$ and $G_{A}^{(T=1)}$.

Scattered electrons generated Cherenkov light in an air medium, which was then focused by ellipsoidal mirrors toward lead shielded 8-inch photomultiplier tubes. A detailed account of the experimental technique and data analysis can be found in $[39,40]$. By using the electron-proton scattering data, the measured asymmetry and the theoretical value of $G_{A}^{(T=1)}$ calculated in [53] gives rise to a value for the strange magnetic form factor of

$$
G_{M}^{s}\left(Q^{2}=0.1\right)=0.37 \pm 0.20(\text { stat }) \pm 0.26(\text { syst }) \pm 0.07(\mathrm{FF})
$$




\subsubsection{A4}

The A4 experiment made measurements of both forward-angle and backward-angle scattered electrons at the Mainzer Mikrotron accelerator facility (MAMI) in Mainz, Germany. For both phases, they measured a parity-violating asymmetry by scattering a $20 \mu \mathrm{A}$ polarized beam of electrons off a $10 \mathrm{~cm}$ liquid hydrogen target at low beam energies less than $1 \mathrm{GeV}$.

The forward angle measurements were conducted for two values of four momentum transfer $Q^{2}=0.108,0.23 \mathrm{GeV}^{2}$ with corresponding beam energies of $\mathrm{E}=570.4,854.3$ $\mathrm{MeV}$. The elastically scattered electrons were detected by a large acceptance fast $\mathrm{PbF}_{2}$ calorimeter that covered a range of scattering angles $30^{\circ}<\theta_{e}<40^{\circ}$ and a solid angle of $\Delta \Omega=0.62 \mathrm{sr}$. From their asymmetry measurements, the results for the strange quark form factor contributions are

$$
\begin{gathered}
Q^{2}=0.108 \mathrm{GeV}^{2}: G_{E}^{s}+0.106 G_{M}^{s}=0.071 \pm 0.036 \\
Q^{2}=0.230 \mathrm{GeV}^{2}: G_{E}^{s}+0.225 G_{M}^{s}=0.039 \pm 0.034
\end{gathered}
$$

where the quoted errors are from statistics and systematics [41, 42].

The backward angle measurement was conducted at $Q^{2}=0.22 \mathrm{GeV}^{2}$ with the same experimental apparatus and covered a range of scattering angles $140^{\circ}<\theta_{e}<150^{\circ}$ with the same solid angle. In order to get the 4-momentum transfer to agree with the forward angle measurement, the beam energy used was $\mathrm{E}=315.1 \mathrm{MeV}$. The linear combination of strange vector form factors obtained was $G_{M}^{s}+0.26 G_{E}^{s}=-0.12 \pm$ $0.011 \pm 0.11$ and can be used with the A4 forward angle measurements to disentangle the form factors [43], as shown in Figure 5.3

$$
\begin{aligned}
& G_{E}^{s}\left(Q^{2} \approx 0.22\right)=0.050 \pm 0.038(\text { stat }) \pm 0.019(\text { syst }) \\
& G_{M}^{s}\left(Q^{2} \approx 0.22\right)=-0.140 \pm 0.11(\text { stat }) \pm 0.11(\text { syst })
\end{aligned}
$$

\subsubsection{HAPPEx}

The original HAPPEx took place in 1998-1999 and aimed to make a measurement of the parity-violating asymmetry of electrons scattered off a liquid hydrogen target at a momentum transfer of $Q^{2}=0.477 \mathrm{GeV}^{2}$. It was conducted in Hall A of Jefferson Labs, where an electron beam with energy of $3.2 \mathrm{GeV}$ was aimed at a $15 \mathrm{~cm}$ long 


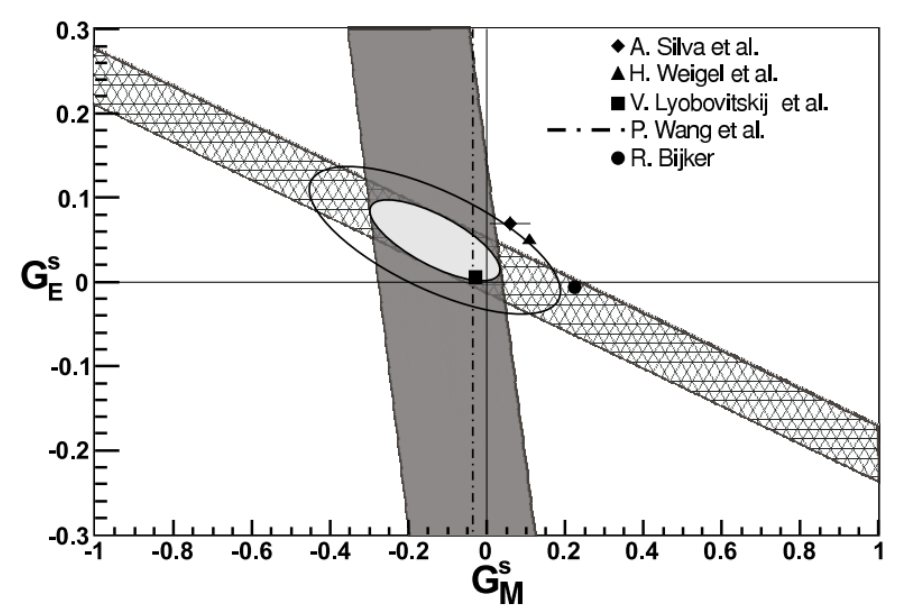

Figure 5.3: One sigma results for the linear combination $G_{M}^{s}+\eta G_{E}^{s}$ for A4 backward (solid band) and forward angle (hatched band) measurements at $Q^{2} \approx 0.22 \mathrm{GeV}^{2}$. The ellipses represent the $68 \%$ and $95 \%$ confidence levels. Also show are several theoretical predictions.

unpolarized liquid hydrogen target. Elastic electrons with a scattering angle of $12.3^{\circ}$ were guided by the HRS toward a special integrating detector and DAQ.

Data taking was split into two periods, at the end of 1998 and at the beginning of 1999. In 1998 the experiment used a $100 \mu \mathrm{A}$ beam with $38 \%$ polarization, while the data taken in 1999 used a $35 \mu \mathrm{A}$ beam current with a 70\% degree of polarization. From the measured asymmetry, the following combination of strange form factors were obtained

$$
G_{E}^{s}+0.392 G_{M}^{s}=0.014 \pm 0.020(\text { stat }) \pm 0.010(\text { syst }) .
$$

A second generation HAPPEx was conducted in 2004-2005 at a four momentum transfer value of $Q^{2} \approx 0.1 \mathrm{GeV}^{2}$. HAPPEx-II had two phases, each with a different target. They are referred to as HAPPEx-H, which used a liquid hydrogen target, and HAPPEx-He, which used a high pressure helium gas target. While they both used the same experimental apparatus as the original experiment, the scattering angle of $\approx 6^{\circ}$ required the use of a septum magnet to guide the elastic peak toward the Hall $\mathrm{A}$ high-resolution spectrometers. Because the helium target is spinless, the HAPPExHe measurement was used to isolate the contribution from the strange electric form factor $G_{E}^{s}[45]$. 
The HAPPEx-H result provided access to $G_{E}^{s}+0.09 G_{M}^{s}=0.007 \pm 0.011 \pm 0.006$ at $Q^{2}=0.109 \mathrm{GeV}^{2}$. By extrapolating the result from HAPPEx-He

$$
G_{E}^{s}\left(Q^{2}=0.077\right)=0.002 \pm 0.014 \pm 0.007
$$

up to $Q^{2}=0.1 \mathrm{GeV}^{2}$ both strange form factors can be calculated from a best fit of the entire data set:

$$
\begin{aligned}
G_{E}^{s} & =-0.005 \pm 0.019 \\
G_{M}^{s} & =0.18 \pm 0.027
\end{aligned}
$$

\subsubsection{G0}

The G0 experiment was conducted by a large collaboration in Hall C of Jefferson Lab. It consisted of using CEBAF's polarized beam of electrons to interact with liquid hydrogen and deuterium targets to measure a parity violating asymmetry. Like the A4 experiment, the measurements were done in two phases, referred to as forward-angle and backward-angle.

The first aimed at detecting elastically scattered protons that were kicked out of the target by recoil, guided by a superconducting toroidal spectrometer and detected by an array of specially designed scintillator detectors. The second phase of the experiment reversed the apparatus relative to the beam direction and aimed at detecting backward-angle scattered electrons off the liquid hydrogen target as well as quasi-elastic electrons scattered off the deuterium target.

Utilizing a $40 \mu \mathrm{A}$ polarized beam at an energy of $3.03 \mathrm{GeV}$, the spectrometer guided forward-angle recoiled protons toward different sections of the detector package, allowing for a simultaneous measurement over a wide range of 4-momentum transfer values [46]. The forward angle asymmetries measurements provided access to a linear combination of the strange form factors $G_{E}^{s}+\eta G_{M}^{s}$ for $Q^{2}=[0.12,1.0]$ $\mathrm{GeV}^{2}$, which make up most of the data points in Figure 5.1 .

The backward-angle phase of the experiment aimed at measuring the strange magnetic form factor $G_{M}^{s}$ and the axial form factor $G_{A}$ at two values of momentum transfer $Q^{2}=0.22,0.63 \mathrm{GeV}^{2}$. For these measurements, a $60 \mu \mathrm{A}$ polarized beam at two different energies $\mathrm{E}=359,684 \mathrm{MeV}$ was aimed at both the liquid hydrogen and deuterium targets. 


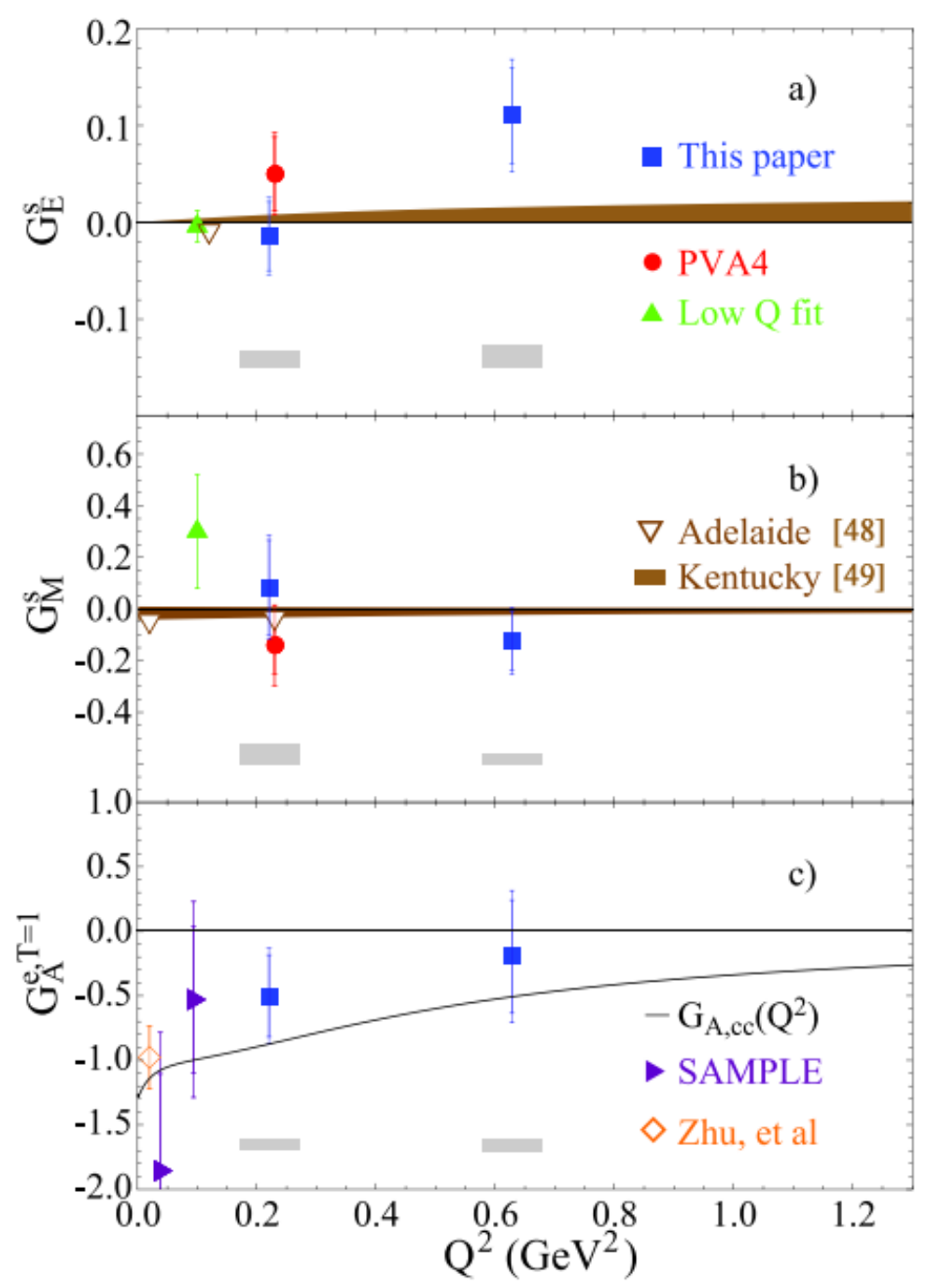

Figure 5.4: The three plots above summarize the results of the G0 forward- and backward-angle measurements of the strange and anapole form factors (blue data point). The shaded bars represent global systematic uncertainties for those points. Included are results from A4 and SAMPLE, as well as low Q fit points from [48]. Some theoretical calculations are also included $[49,50]$. 
The asymmetry measured with the liquid hydrogen target was used to isolate the magnetic strange vector form factor $G_{M}^{s}$. The deuterium measurements were used to find the axial contributions $G_{A}$ to the parity-violating asymmetry. By combining the forward and backward angle results at the corresponding values of four-momentum transfer, all three form factors were determined

$$
\begin{aligned}
G_{E}^{s}\left(Q^{2}=0.22\right) & =-0.014 \pm 0.036 \pm 0.018 \pm 0.018 \\
G_{M}^{s}\left(Q^{2}=0.22\right) & =0.083 \pm 0.183 \pm 0.085 \pm 0.078 \\
G_{A}^{e}\left(Q^{2}=0.22\right) & =-0.501 \pm 0.317 \pm 0.193 \pm 0.087 \\
G_{E}^{s}\left(Q^{2}=0.63\right) & =0.110 \pm 0.049 \pm 0.029 \pm 0.024 \\
G_{M}^{s}\left(Q^{2}=0.63\right) & =-0.124 \pm 0.110 \pm 0.061 \pm 0.032 \\
G_{A}^{e}\left(Q^{2}=0.63\right) & =-0.197 \pm 0.425 \pm 0.257 \pm 0.095
\end{aligned}
$$

where the errors are from statistics, systematics and uncertainties in the nucleon form factors, respectively.

As part of their final analysis, the G0 collaboration produced Figure 5.4, which shows values found for $G_{E}^{s}, G_{M}^{s}$ and $G_{A}^{e}$ by using the entire set of experimental world data [47]. The plots show how these values trend with $Q^{2}$ and also includes several theoretical predictions. The values used for the low $\mathrm{Q}$ fit come from a global analysis of all data at that four-momentum transfer [48], discussed in the next section.

\subsubsection{Summary of Measurements at $Q^{2} \approx 0.1 \mathrm{GeV}^{2}$}

Figure 5.5 shows the world data for $Q^{2} \approx 0.1 \mathrm{GeV}^{2}$ from SAMPLE, HAPPEx, A4 and the G0 collaborations. Each experiment was successful in measuring either a linear combination of form factors, or in the case of HAPPEx-He, the electric strange form factor itself. Each result is represented as a different colored band spread over a one-sigma range in the $\left(G_{E}^{s}, G_{M}^{s}\right)$ plane. The G0 band is a combination of the three lowest $Q^{2}$ bins, ranging from 0.122 to $0.136 \mathrm{GeV}^{2}$. The best fit analysis conducted by Liu et al. provides a new set of values for the strange quark form factor contributions $[48]$

$$
\begin{array}{llc}
G_{E}^{s} & = & -0.006 \pm 0.016 \\
G_{M}^{s} & = & 0.33 \pm 0.21 .
\end{array}
$$




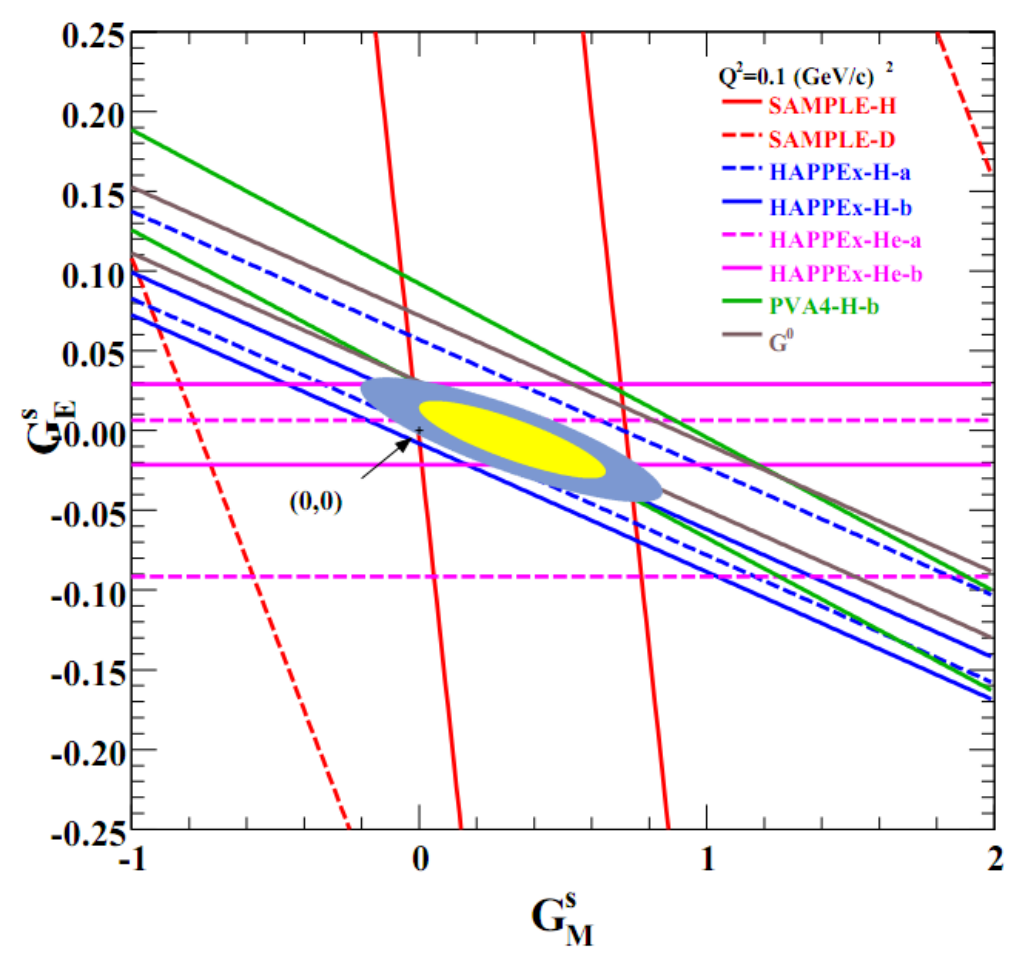

Figure 5.5: Results for all measurements of strange form factors from several parity violation experiments conducted at $Q^{2} \approx 0.1 \mathrm{GeV}^{2}$. The yellow and blue ellipses are $68 \%$ and $95 \%$ confidence level fits. Note that zero strangeness $\left(G_{E}^{s}=G_{M}^{s}=0\right)$ is at the edge of the $95 \%$ C. L. contour.

A similar world data fit was conducted by Young et al. which aimed at extracting the strange form factors as well as the anapole form factors. Their analysis was also consistent with zero strangeness and anapole contributions to the nucleon form factors [51]. They determined that at the $95 \%$ confidence level, strange quarks contribute less than $5 \%$ of the mean-square charge of the proton and less than $6 \%$ of the magnetic moment.

\subsection{Other Applications of PVES Results}

While the parity violating electron scattering experiments discussed so far have aimed at determining strangeness contributions, the asymmetry measurements can also be used to find quark weak charge couplings [52]. At small values of momentum transfer, 


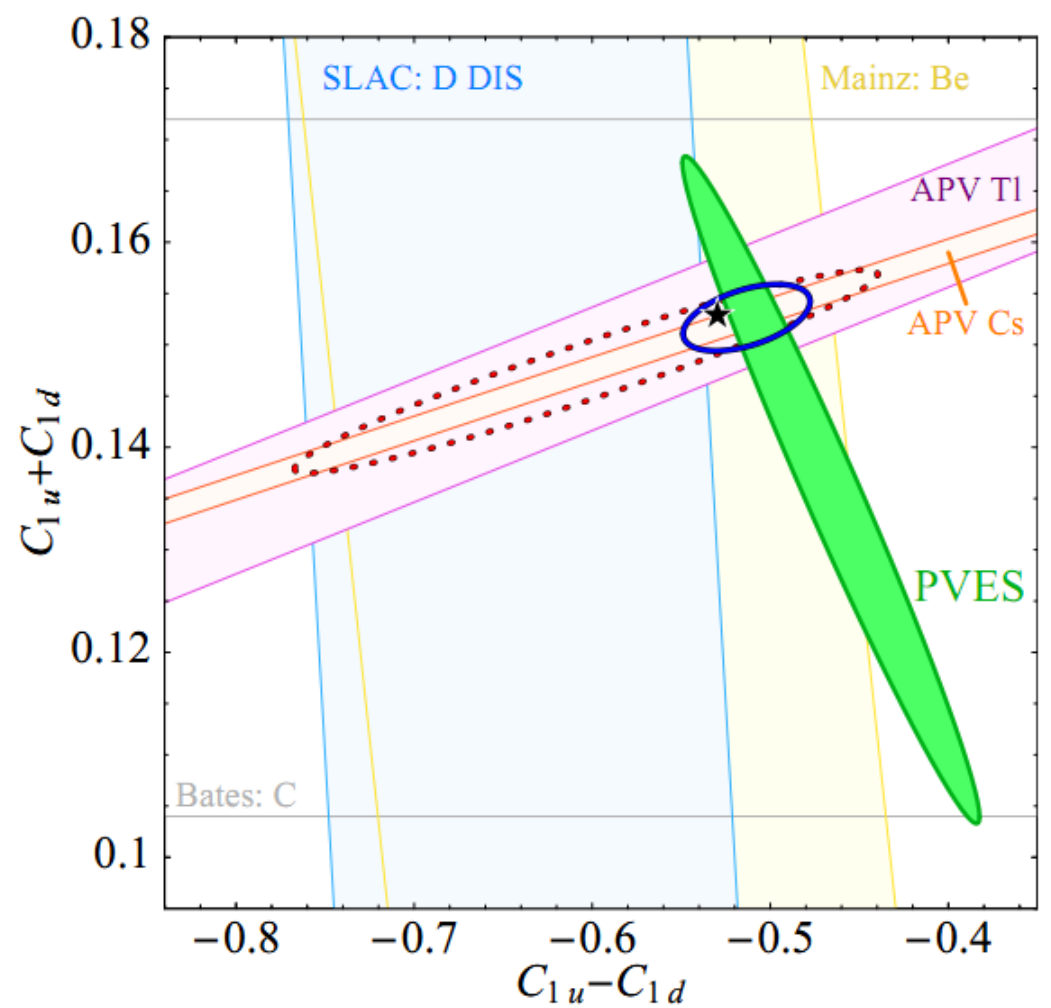

Figure 5.6: The world data of the neutral weak effective couplings. The dotted contour shows a $95 \%$ confidence level fit of all the data before including results from parity violating electron scattering experiments. The filled contour represents the new constraint provided by PVES results, while the blue contour shows the 95\% CL fit of the entire data set.

the asymmetry can be expressed as

$$
A_{L R} \approx A_{0}\left[Q_{w e a k}^{p} Q^{2}+B_{4} Q^{4}+\ldots\right]
$$

where $A_{0}=-G_{F} /(4 \pi \alpha \sqrt{2}), Q_{\text {weak }}^{p}$ is the weak charge of the proton, $Q$ is momentum transfer and $B_{4}$ is a higher-order term which accounts for hadronic structure and includes strange quarks. The weak charge of the proton can be expressed in terms of the quark weak charges as

$$
Q_{w e a k}^{p}=-2\left(2 C_{1 u}+C_{1 d}\right)
$$

$C_{1 u}$ and $C_{1 d}$ are parameters that describe how the weak force interacts with the up and down quarks in the proton when described by a four-point contact interaction. 


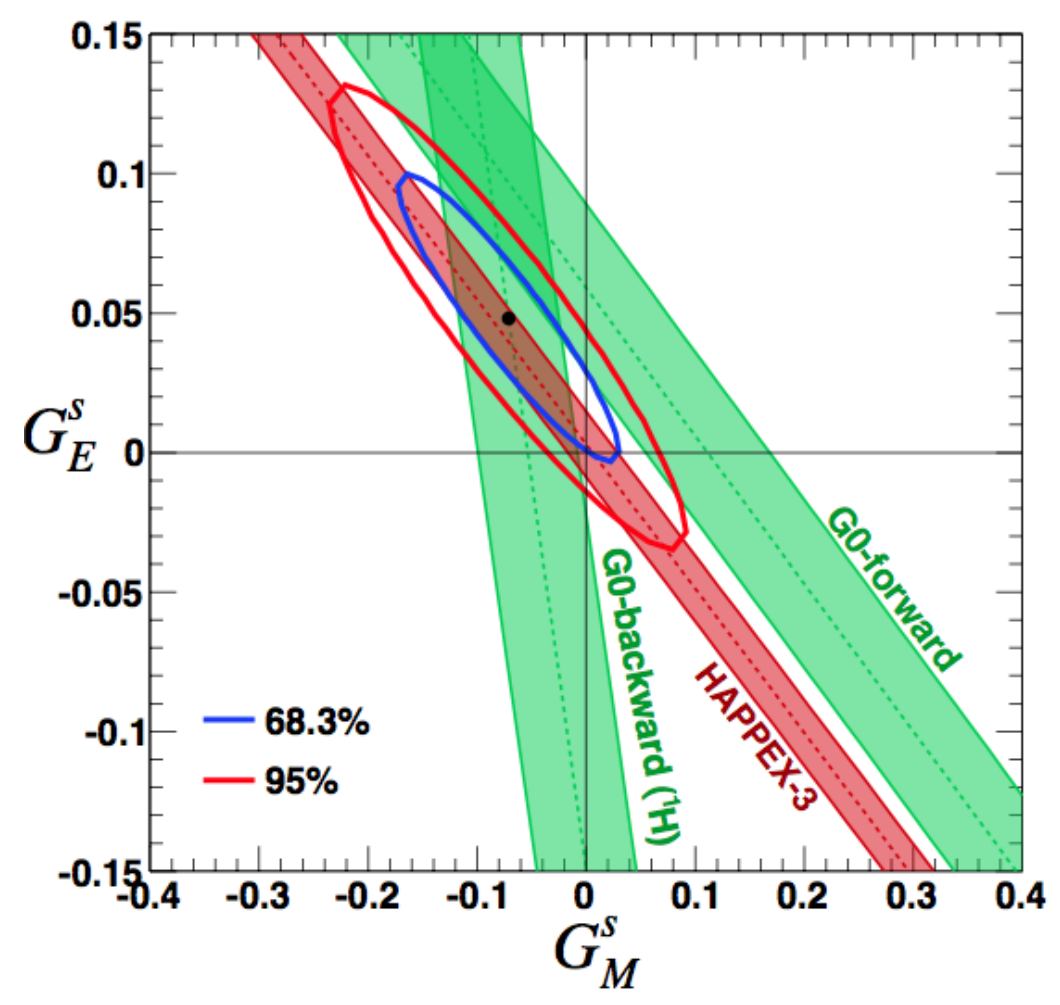

Figure 5.7: Current experimental constraints on the strange form factors when considering all the existing data at $Q^{2} \approx 0.62 \mathrm{GeV}^{2}$. These include bands from the HAPPEx-III and G0 forward- and back-angle measurements.

Figure 5.6 shows the impact of the PVES results on a global fit of the electroweak parameters $C_{1 u, d}$. By including the green ellipse to constrain the previous fit from APV and DIS data (dashed contour), the area of the new $95 \%$ confidence level fit (solid contour) is smaller by a factor of 5 .

The values obtained for $C_{1 u}+C_{1 d}$ and $C_{1 u}-C_{1 d}$ through this fit are in excellent agreement with the latest Standard model values, marked with a star in Figure 5.6. This analysis severely limits the possibilities of new physics beyond the Standard Model below a mass scale of $\approx 1-5 \mathrm{TeV}$ [52]. Future high-precision measurements such as the Q-weak experiment being conducted in Hall C of Jefferson Lab will set even further constraints on the allowed values of $C_{1 u, d}$. 


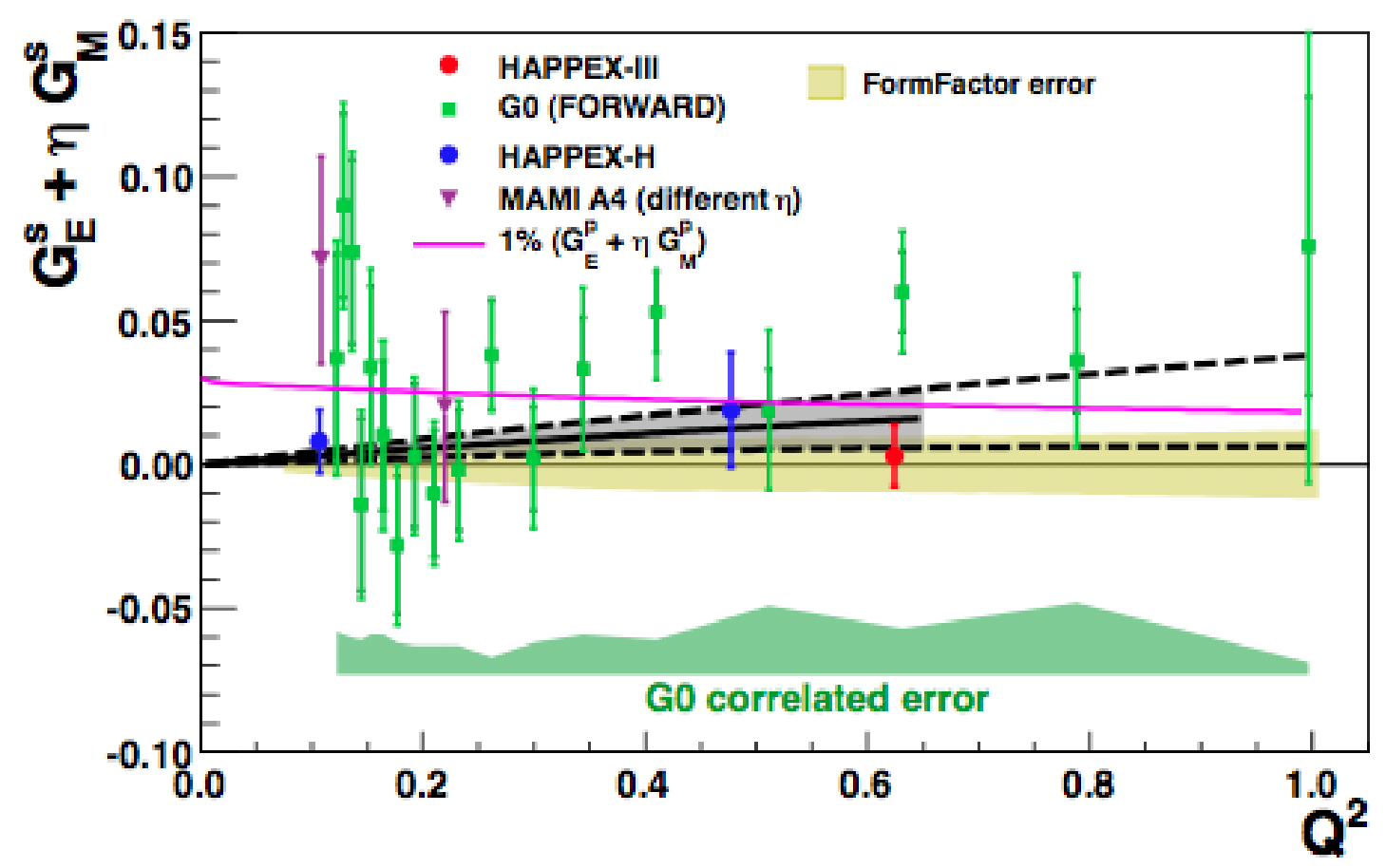

Figure 5.8: Plotted is the entire set of world data for forward-angle measurements of linear combinations of strange vector form factors. This is an updated version of Figure 1.9 and includes the HAPPEx-III measurement (red data point), which further constrains the linear fit shown, moving it closer to zero.

\subsection{Implications of HAPPEx-III Result}

The HAPPEx-III measurement was conducted at a high four momentum transfer in order to explore a growing positive trend of $G_{E}^{s}+\eta G_{M}^{s}$ at higher values of $Q^{2}$. Figure 5.7 shows all published data at $Q^{2} \approx 0.62 \mathrm{GeV}^{2}$, including G0 backward and forward angle as well as our current measurement. While the G0 results show considerable contributions from both $G_{E}^{s}$ and $G_{M}^{s}$. After combining all three measurements by adding the HAPPEx-III result and accounting for uncertainties in $A_{N S}$, new values are found for the strange vector form factors

$$
\begin{aligned}
G_{E}^{s} & =0.047 \pm 0.034 \\
G_{M}^{s} & =-0.070 \pm 0.067
\end{aligned}
$$

for which both the $68 \%$ and $95 \%$ C. L. fits are consistent with zero.

Figure 5.8 shows the entire set of world data from forward-angle experiments 


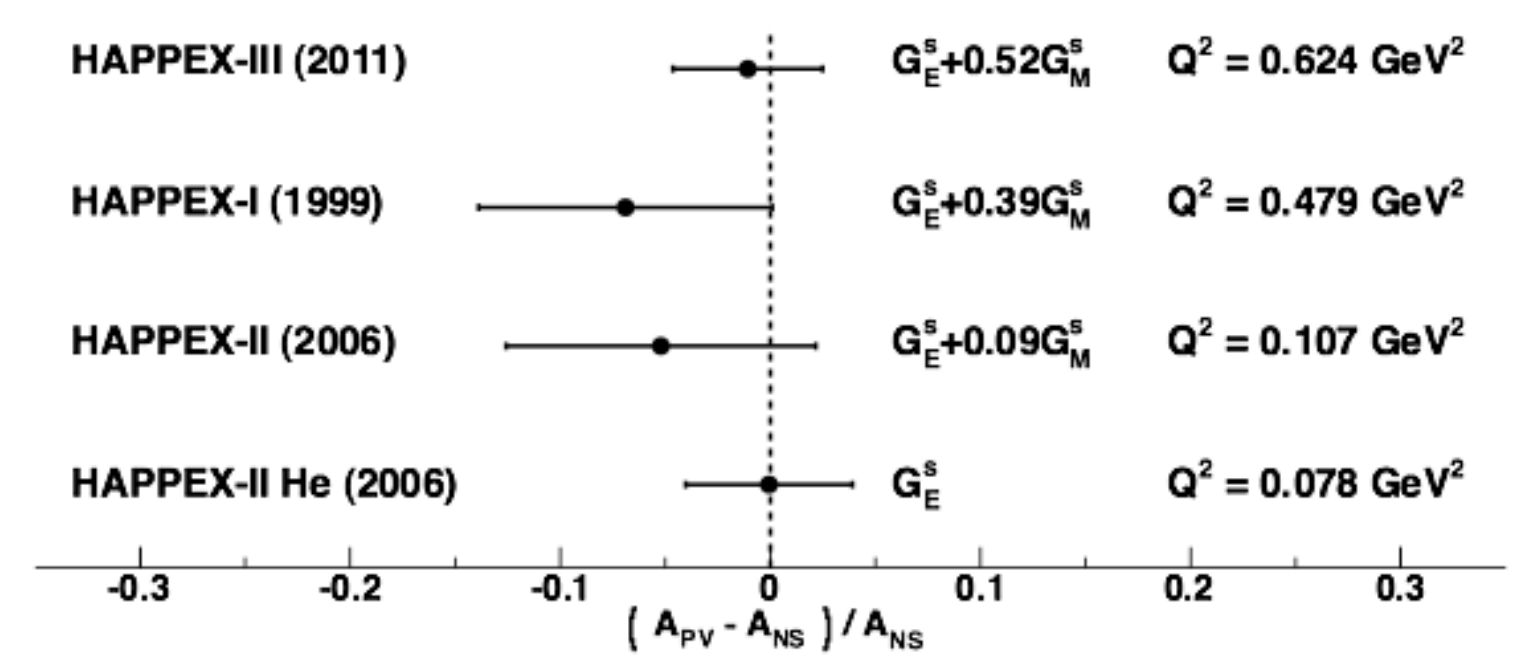

Figure 5.9: Difference error when comparing the 'no strangeness' asymmetry to the measured physics asymmetry for all HAPPEx results. All these measurements are consistent with zero strangeness.

conducted at MAMI and Jefferson Labs. By adding the HAPPEx-III result to this data, the trend of strange quark contribution is further constrained and now overlaps with the uncertainty in $A_{N S}$. The fit shown includes all published data and takes into account the correlated uncertainties in the G0 measurements. The shaded region is the one sigma error of a leading order fit in which $G_{M}^{s}$ is kept constant and $G_{E}^{s}$ is $Q^{2}$-dependent.

From the PV experiments conducted to gain information about the strangeness of the nucleon form factors, all four HAPPEx measurements are the most accurate determinations of strange quark contribution. Figure 5.9 shows the fractional deviation of the measured values from the expected asymmetry for no strangeness. Within the error bars, they are all consistent with zero strangeness. While most theories do not make accurate predictions at high momentum transfers [54], these results show that there is no anomalous $\mathrm{Q}^{2}$-dependent increase in the contribution of strangeness. 


\subsection{The Future of Parity Violation Expriments}

\subsubsection{A4-III Results and Developments}

The A4 collaboration at MAMI also conducted a PV asymmetry forward-angle measurement at a high value of four-momentum transfer. For this version of their experiment, they once again used the same setup as for their previous measurements. Using a beam energy of $\mathrm{E}=1508 \mathrm{MeV}$, the scattered flux was detected at a $35^{\circ}$ angle in order to obtain a four-momentum value of $Q^{2}=0.62 \mathrm{GeV}^{2}$. The data was taken in 2009 and analysis was completed in the Fall of 2011. The experiment collected 600 hours of asymmetry data with a beam polarization of $85 \%$ and achieved an error of about $5 \%$.

While preliminary results from A4-III were presented by Sebastian Baunack at a recent workshop in Italy [56], a final result is still in the works. The value presented for the linear combination of strange vector form factors is $G_{E}^{s}+0.628 G_{M}^{s}=0.067 \pm 0.030$. Once the final result is released, further analysis must be conducted with the high $Q^{2}$ data to put further constraints on the values of $G_{E}^{s}$ and $G_{M}^{s}$.

The A4 collaboration also began taking data on a new set of kinematics in August of 2011. Similar to the SAMPLE experiment, the A4-IV measurement takes place at a backward scattering angle with a beam energy of $\mathrm{E}=210 \mathrm{MeV}$ and a fourmomentum transfer of $Q^{2}=0.1 \mathrm{GeV}^{2}$. The result will put further constraints on the current values of $G_{E}^{s}$ and $G_{M}^{s}$ discussed in Section 5.3.5.

\subsubsection{PREX-II}

A proposal for a followup to PREx was submitted and approved to be conducted in Hall A of Jefferson labs. PREx-II will take advantage of all the knowledge gained about the experimental apparatus during the first run in order to decrease the current statistitical error on the asymmetry of $9 \%$.

Due to several technical problems, PREx was only able to collect about $15 \%$ of the planned statistics. This was mostly due to the failure of a component in the vacuum coupling of the scattering chamber. There was a large radiation dose inside the hall that caused certain systems to malfunction. While there were also some problems with the lead target, these were mainly addressed throughout the production run and 


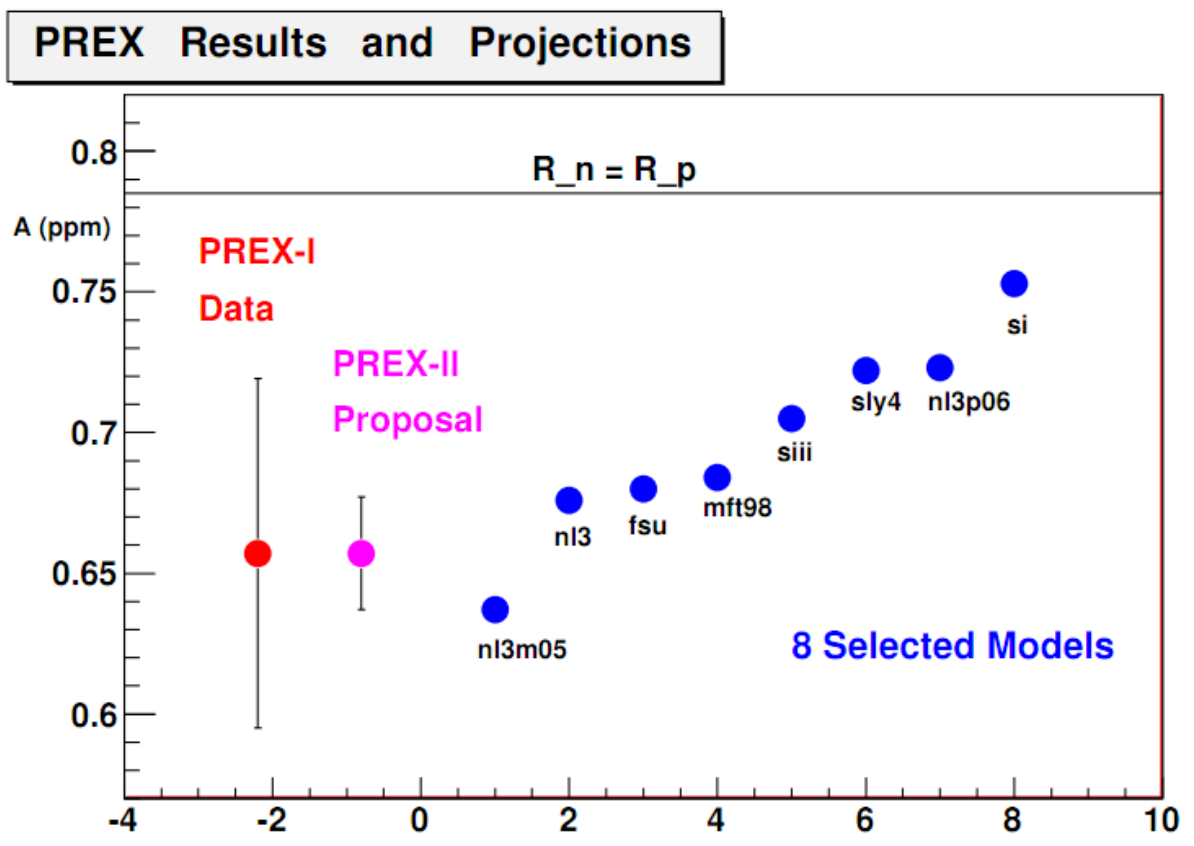

Figure 5.10: The red data point shows the final asymmetry result of the PREx experiment. The pink data point shows the proposed error for a PREx-II result. Also shown are theoretical values from 8 selected models. The $R_{n}=R_{p}$ line represents the asymmetry if there was no neutron skin.

the collaboration is certain the target design is robust.

To address the damaging radiation, plans are underway to add proper shielding to certain electronics, as well as to the collimators. Several options are currently available and are discussed in detail in the PREx-II proposal document [55]. With 25 more days of efficient data taking, PREx-II will gather enough statistics to reach the original proposed error goal of $1 \%$ for $R_{N}$, the RMS radius of the Lead-208 nucleus. Figure 5.10 shows how this new result would help put further constraints on several model predictions.

\subsection{3 $12 \mathrm{GeV}$ MOLLER}

The MOLLER (Measurement Of a Lepton Lepton Electroweak Reaction) experiment was proposed in 2010 at Jefferson Lab. It will take advantage of the upcoming $12 \mathrm{GeV}$ upgrade to make an ultra precise measurement of the weak mixing angle. The experiment will measure the parity violating asymmetry in polarized electron- 


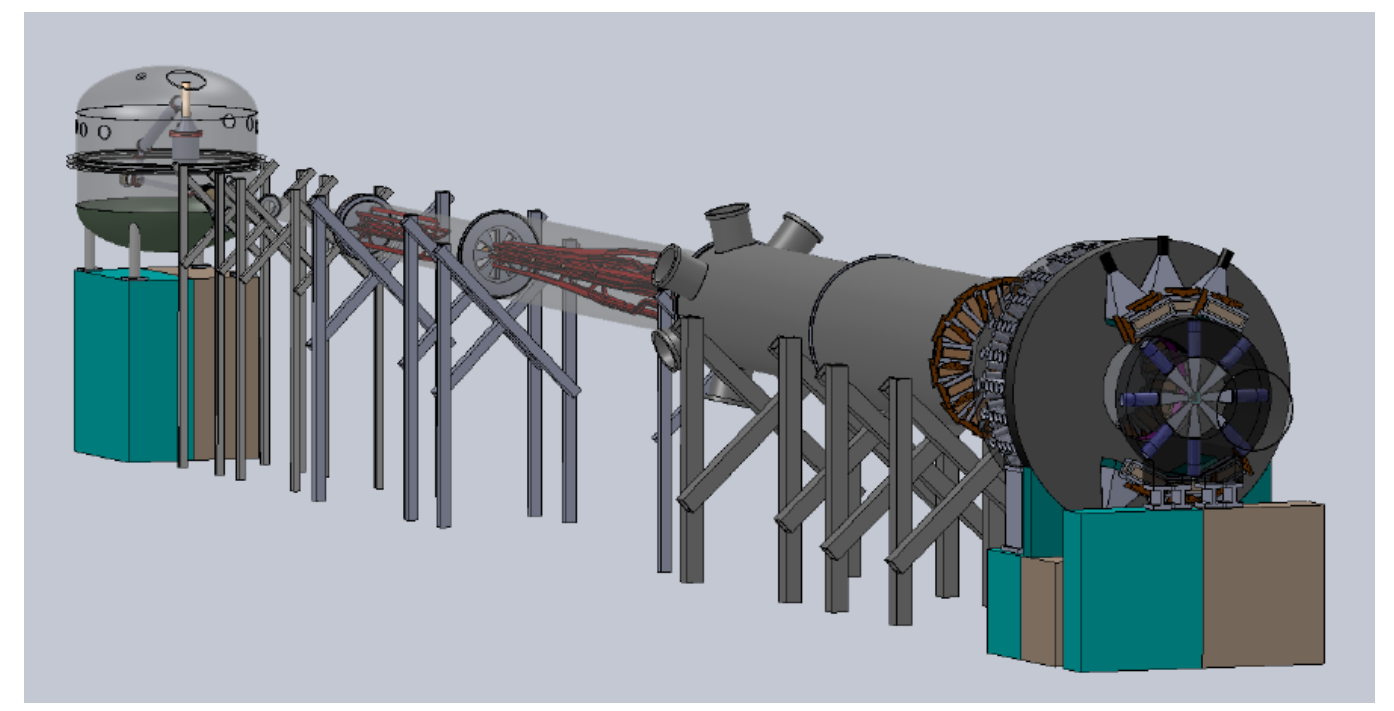

Figure 5.11: Layout of the MOLLER experiment, to be conducted in Hall A at Jefferson Labs. From left to right, it shows the target chamber, toroidal spectrometer and detector package.

electron (Moller) scattering with a precision of $0.73 \mathrm{ppb}$ at the proposed kinematics. Figure 5.11 shows a 3 -D schematic of the proposed layout of the experiment, from the target chamber on the left to the detector package on the right.

The experiment will run in Hall A using a $11 \mathrm{GeV}$ polarized beam incident on a $1.5 \mathrm{~m}$ long unpolarized liquid hydrogen target. Elastically scattered electrons will be guided by two back-to-back toroid magnets toward a ring of detectors that cover the entire azimuthal range of angles. A set of primary acceptance collimators located before the first toroid will make sure that only one of the scattered electrons from the Moller reaction will reach the detectors. There is an odd number of collimator petals so that the asymmetric configuration will allow for this kind of single acceptance.

The toroidal spectrometer is specially designed so that (a) the electrons that clear the acceptance collimators are spread to cover the full azimuthal range and (b) are focused into thin ring of detectors $\approx 28 \mathrm{~m}$ from the target center, where there will be clear separation from the elastic e-p peak. These specifications will allow for a relatively background free measurement of the e-e elastic peak.

As with previous PV experiments conducted at Jefferson Labs, being able to measure this sub-ppm level asymmetry will depend on accurate calibration and monitoring of beam properties is essential. With the proper controls on systematic errors, the 
proposed result for this experiment will provide a measurement of the weak charge of the electron to a fractional accuracy of $2.3 \%$, lower than any such measurement proposed to date.

\subsection{Conclusion}

The HAPPEx-III measurement was successful in ruling out large contributions from strange quarks to the nucleon form factors at a four-momentum transfer of $Q^{2} \approx 0.62$ $\mathrm{GeV}^{2}$. This result shows that the linear combination of strange vector form factors is consistent with zero withing the experimental uncertainty. In order to conduct experiments with higher precision, considerable work must be done to understand the nucleon electromagnetic form factors, which contribute considerable uncertainty to our results. Also, further theoretical calculations through Lattice QCD need to be refined.

All the new technologies created by the HAPPEx collaboration to achieve the systematic error goal of $2 \%$ will be very helpful to the experiments discussed in the previous section. The upgrades done to the Compton polarimeter and the source optics are already being used by the Qweak experiment in Hall C of Jefferson Labs to measure the weak charge of the proton. 


\section{APPENDIX A}

\section{LINEARITY OF PHOTOMULTIPLIER TUBES}

In Chapter 3, the concept of detector linearity was introduced. Before a PMT can be used as a physics detector, it is essential to make sure that its output is understood. For this purpose, an experimental test setup was created and numerous tests were conducted at a wide range of input currents and gain levels (HV values). This Appendix summarizes the results for one of the many 2-inch PMTs used as part of the luminosity monitor and the PREx detector package.

PMTs can have very large non-linearities for very high input currents because of saturation effects. This behavior can be seen in the linearity test results shown in Figures A.1, A.2 and A.3. The three tests were conducted at different input currents while the PMT gain was kept at the same value of $\mathrm{HV}=700$ (gain factor approx . While the PMT is fairly linear for the $\mathrm{I}=20 \mathrm{nA}$ input current $(\approx 4 \%)$, the results get worse as the current is increased.

Throughout our tests, we found that such non-linearities could also occur at very low and very high gain levels. We showed that for each input current, there is a range of $\mathrm{HV}$ values in which the detector output is linear to better than $2 \%$. Tables A.1, A.2 and A.3 summarize the results of about 100 tests conducted with a single PMT at three different input photocathode currents. The range of input currents were chosen to closely resemble the high luminosity levels expected when the PMT is being used as a luminosity monitor.

The data in Table A.1 was collected at the same input photocathode current of $10 \mathrm{nA}$. It shows that the PMT meets our linearity requirements for a range of high voltage settings of $\mathrm{HV}=[500,1000](-\mathrm{V})$, which create a PMT output current of $\mathrm{I} \approx$ $[1.6,43] \mu \mathrm{A}$. As the input current is decreased, the PMT is linear at a higher range of voltage values but a similar range of output currents. For an input current of $5 \mathrm{nA}$, the linear range of output current was $\mathrm{I} \approx[0.8,49] \mu \mathrm{A}$, while for an input current 


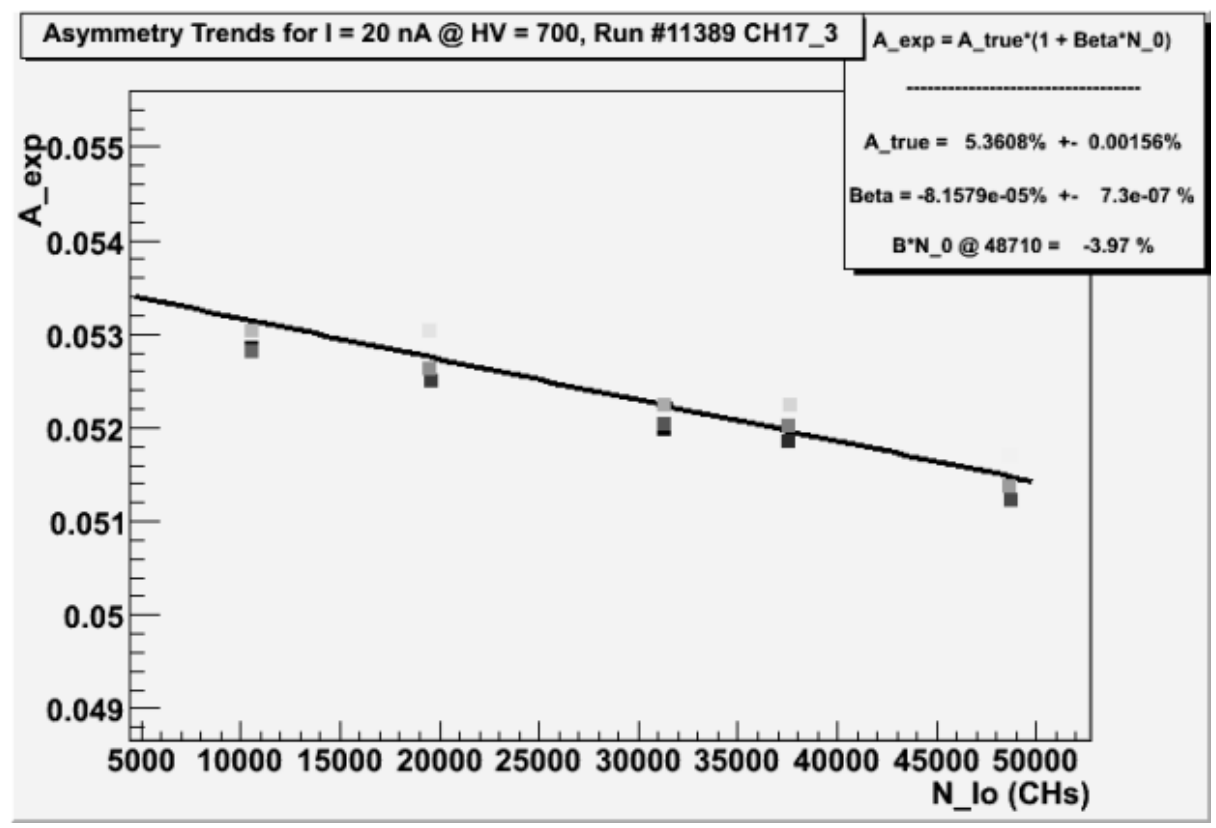

Figure A.1: Linearity test conducted at $\mathrm{HV}=700$ for an input current of $\approx 20 \mathrm{nA}$.

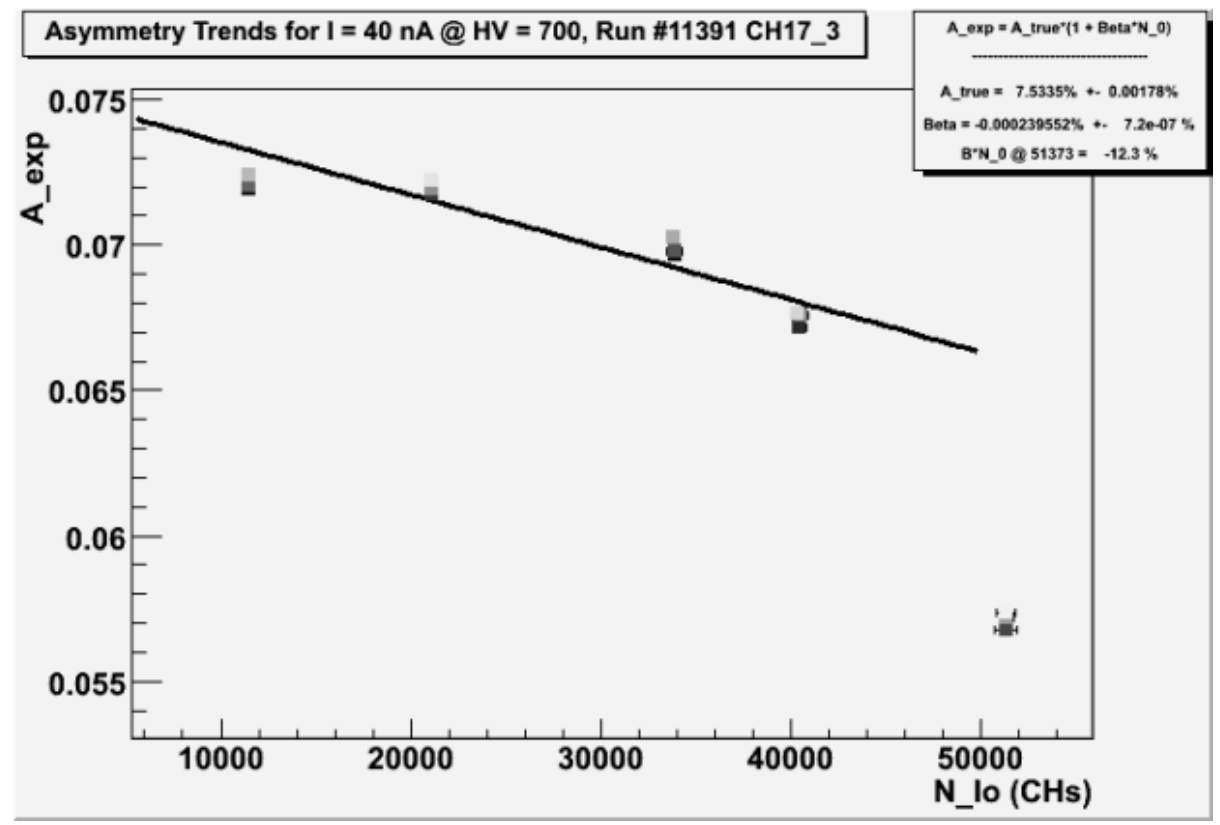

Figure A.2: Linearity test conducted at $\mathrm{HV}=700$ for an input current of $\approx 40 \mathrm{nA}$. 


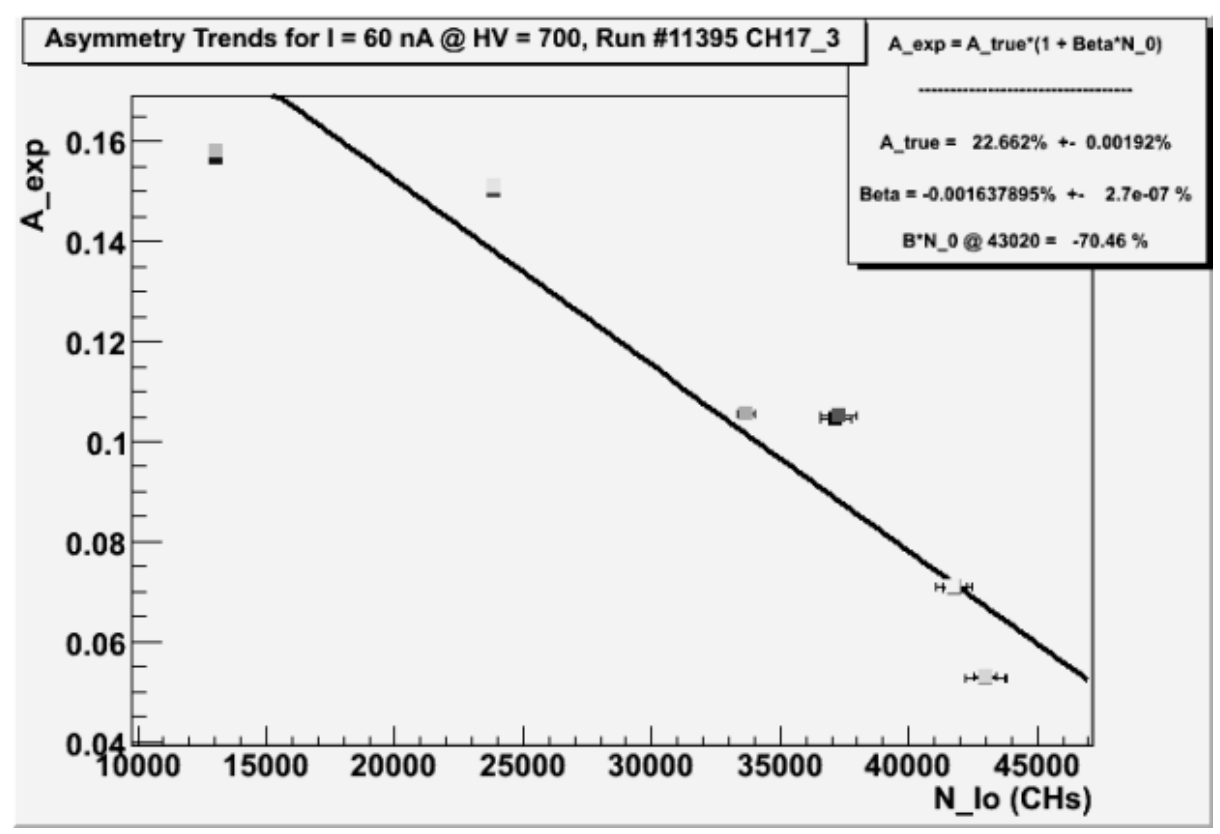

Figure A.3: Linearity test conducted at $\mathrm{HV}=700$ for an input current of $\approx 60 \mathrm{nA}$.

of $1 \mathrm{nA}$, the linear range is $\mathrm{I} \approx[1.4,34] \mu \mathrm{A}$, as is shown in Tables A.2 and A.3. Considering that the method by which we measured PMT gain levels was off at times by a factor of 2 , these PMTs seem to be sufficiently linear for gain factors of 100-4000.

This set of results was shown to be reproducible for the rest of the 2-inch PMTs that were tested. For this reason, we concluded that the best way to assure good PMT linearity was to adjust the PMT gain setting (HV value) so that the output current was about 25-30 $\mu \mathrm{A}$. To facilitate this, the ADCs used to read out the luminosity monitor signals were modified to have a similar maximum range. 


\begin{tabular}{|c|c|c|}
\hline $\mathrm{HV}(-\mathrm{V})$ & $\mathrm{I}(\mu \mathrm{A})$ & $\beta * N_{0}(\%)$ \\
\hline 400 & 0.54 & -5.5 \\
\hline \hline 500 & 1.56 & -0.9 \\
\hline 600 & 3.7 & -0.48 \\
\hline 700 & 7.8 & -0.54 \\
\hline 800 & 15 & 0.28 \\
\hline 900 & 26 & 0.78 \\
\hline 1000 & 43 & 1.32 \\
\hline \hline 1100 & 68 & 2.52 \\
\hline 1200 & 104 & 3.48 \\
\hline 1300 & 155 & 5.2 \\
\hline
\end{tabular}

Table A.1: Sample data set for PMT linearity tests at a photocathode input current of $10 \mathrm{nA}$. The double lines mark the acceptable linearity range.

\begin{tabular}{|c|c|c|}
\hline $\mathrm{HV}(-\mathrm{V})$ & $\mathrm{I}(\mu \mathrm{A})$ & $\beta * N_{0}(\%)$ \\
\hline 400 & 0.26 & -13.85 \\
\hline \hline 500 & 0.76 & -1.96 \\
\hline 600 & 1.8 & -0.39 \\
\hline 700 & 3.8 & -0.34 \\
\hline 800 & 7.2 & -0.21 \\
\hline 900 & 12.6 & -0.26 \\
\hline 1000 & 20.7 & 0.11 \\
\hline 1100 & 32.4 & 0.82 \\
\hline 1200 & 48.5 & 1.29 \\
\hline \hline 1300 & 70 & 2.41 \\
\hline
\end{tabular}

Table A.2: Sample data set for PMT linearity tests at a photocathode input current of $5 \mathrm{nA}$. The double lines mark the acceptable linearity range. 


\begin{tabular}{|c|c|c|}
\hline $\mathrm{HV}(-\mathrm{V})$ & $\mathrm{I}(\mu \mathrm{A})$ & $\beta * N_{0}(\%)$ \\
\hline 700 & 0.75 & -3.15 \\
\hline \hline 800 & 1.4 & -0.075 \\
\hline 900 & 2.5 & 0.23 \\
\hline 1000 & 4.1 & 0.93 \\
\hline 1100 & 6.3 & 0.79 \\
\hline 1200 & 9.4 & 0.51 \\
\hline 1300 & 13.5 & 1.03 \\
\hline 1400 & 18.8 & 0.88 \\
\hline 1500 & 26.3 & 1.27 \\
\hline 1600 & 34 & 1.98 \\
\hline \hline 1700 & 44 & 2.79 \\
\hline
\end{tabular}

Table A.3: Sample data set for PMT linearity tests at a photocathode input current of $1 \mathrm{nA}$. The double lines mark the acceptable linearity range. 


\section{APPENDIX B}

\section{STUDYING NOISE CONTRIBUTIONS WITH LUMIS}

During several test runs conducted in the first half of 2008, the luminosity monitors were installed and used to establish a lower limit on the electronics noise of the experimental setup in Hall A. For these purposes, several configurations of the eight individual luminosity monitors and the ADCs used to read out their signals were attempted. Different neutral density filters $(10 \%, 25 \%$ and $33 \%$ ) were also tried on different lumis. The main configuration had ND filters installed on half of the luminosity monitors because of large input currents. The $10 \%$ filters were useful in avoiding bad linearity for higher gain PMTs which can saturate at lower currents than the rest.

Data was taken with beam of several currents on different targets, including Carbon and different thicknesses of Lead. Current ramps were included to make sure the signals were linear. At very high currents, data taken with a thick Lead target is grossly non-linear, as shown in Figure B.1. While the attenuated lumi signals are fairly linear, the other four get so saturated the signal drops with increasing current. Figure B.2 shows how such non-linearities behave with several other targets. In order to avoid these non-linear regions, it is essential to have proper gain settings for both the PMT and the ADC used to read out its signal.

Once running conditions were established with the main configuration to make sure all the data was sufficiently linear, a few changes were made. Two of the lumis that had a $10 \%$ filter installed, lumis 4 and 8, were blinded in order to completely block the PMT photocathode from the Cherenkov light. This final lumi configuration is shown in Figure B.3. About a week after this change, shielding blocks were installed in front of the three bottom lumis (4,5 and 6). Data from the shielded and unshielded blind lumis was used to understand sources of background affecting the lumi signals. 

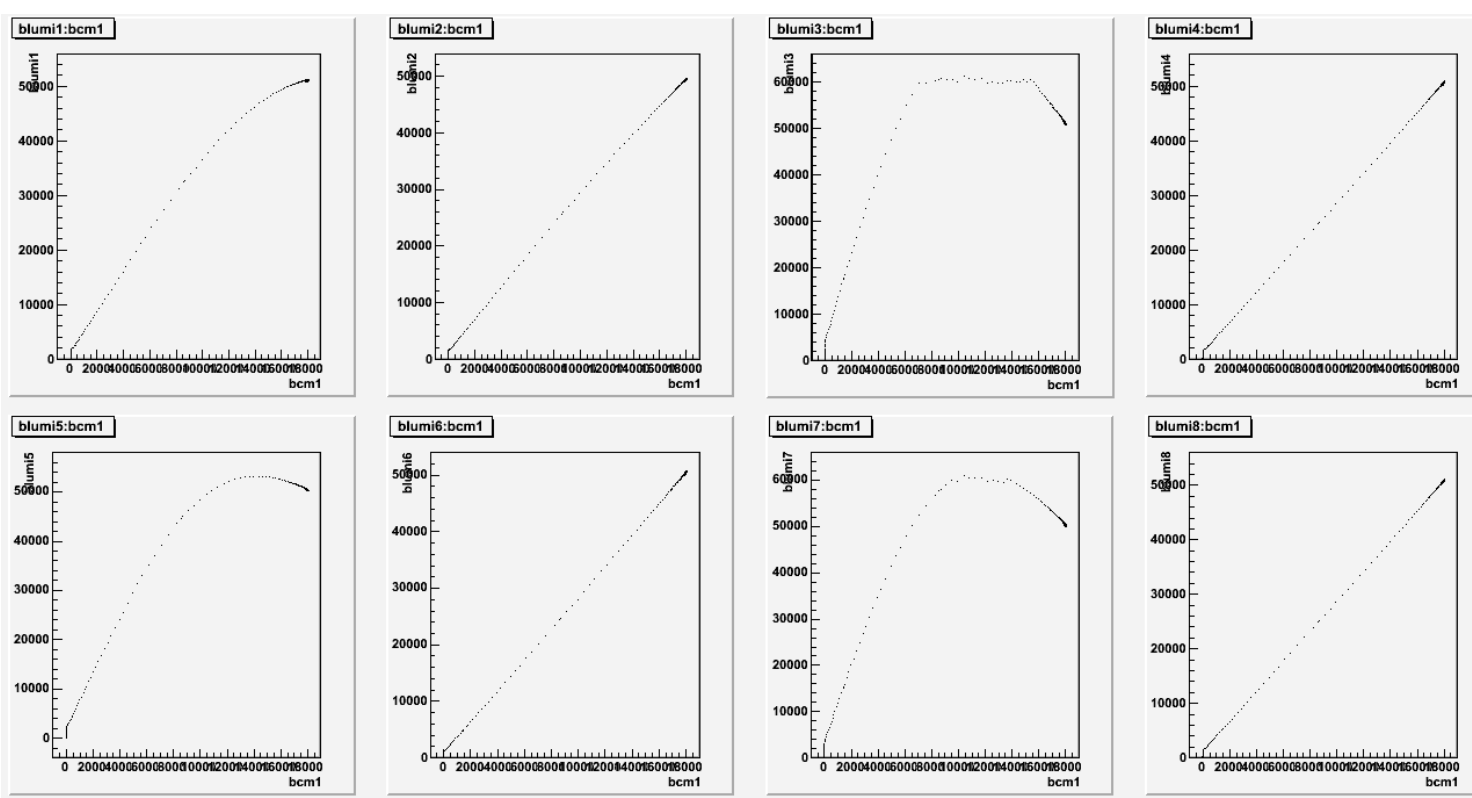

Figure B.1: All eight lumi signals with respect to a beam current monitor signal. For the odd numbered lumis, which had no ND filters installed, the signals are extremely nonlinear and actually drop when the current is increased.
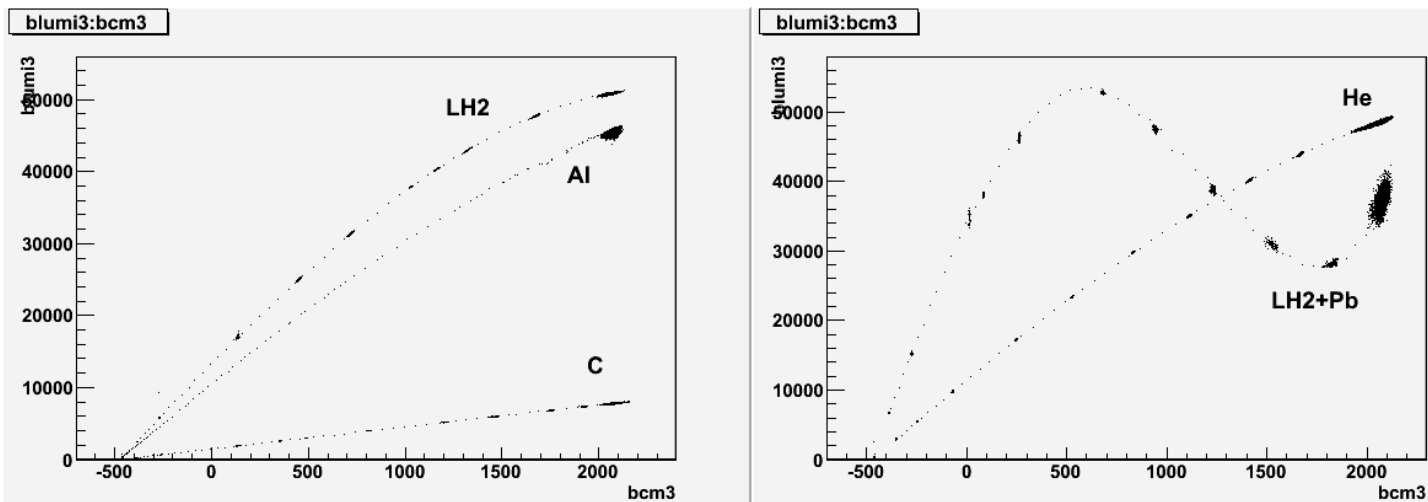

Figure B.2: Both plots show data taken with one of the luminosity monitors during commissioning. When plotted against one of the BCM signals, certain targets give better linear responses than others. 


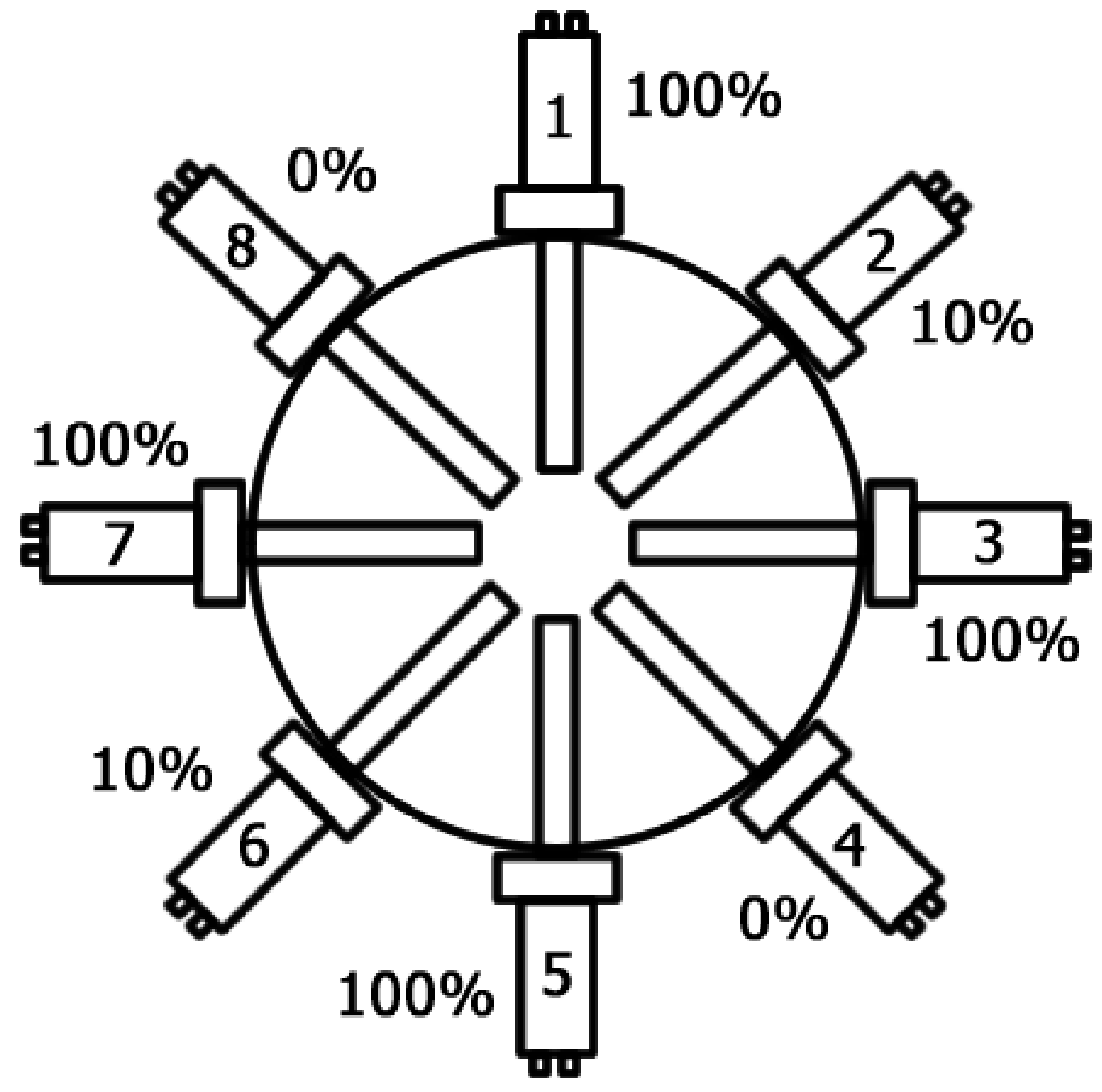

Figure B.3: Luminosity Monitor configuration for the noise level tests. Originally, Lumi 4 had a 10\% filter. Eventually, it was blinded, and later lead bricks were place in front of Lumis 4, 5 and 6 in an attempt to minimize background contributions. 

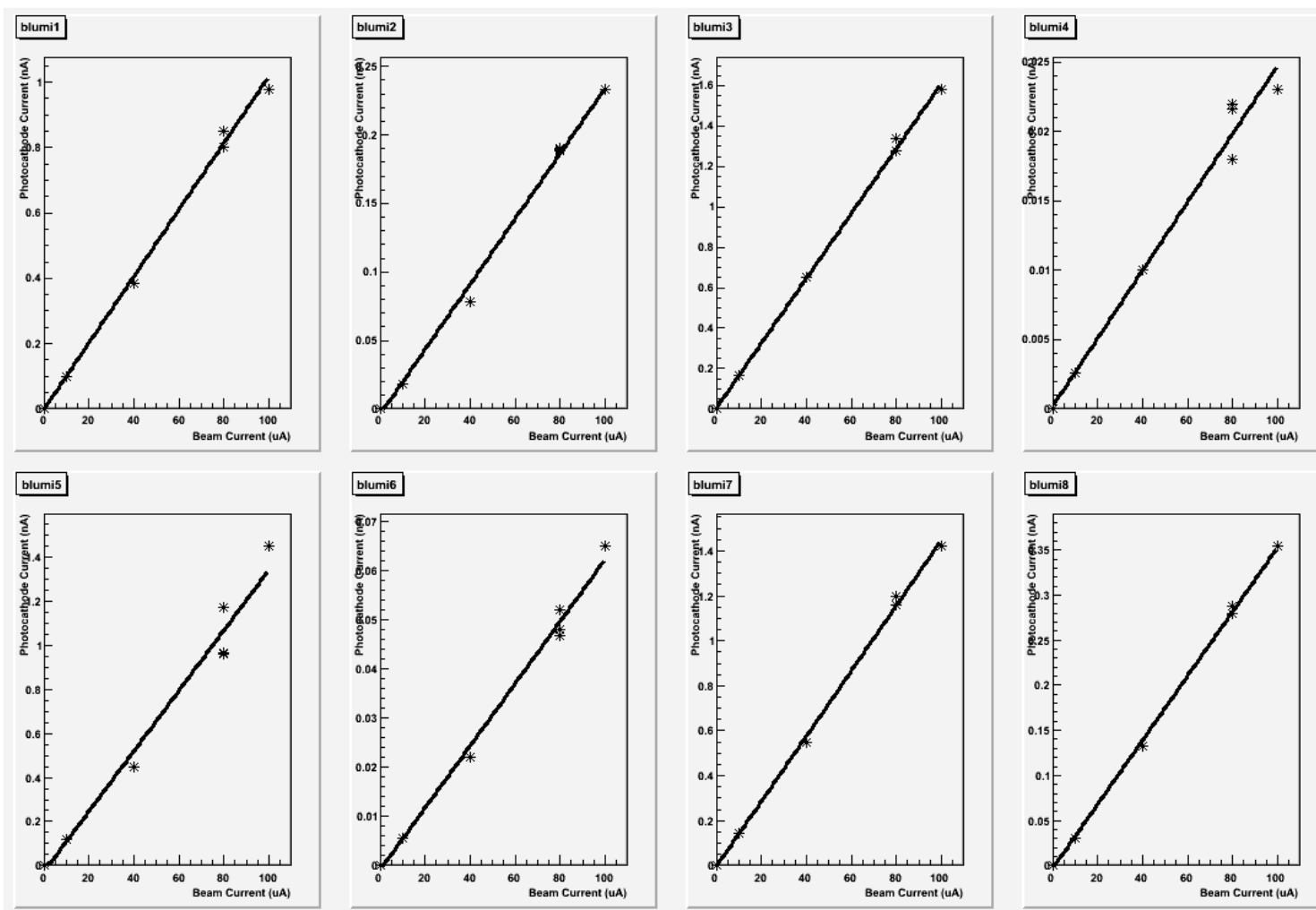

Figure B.4: Summary of luminosity monitor data taken with a carbon target at several beam currents. The lumi signals (y-axis) go up with beam current (x-axis), as expected.

There were several steps in the analysis of the lumi data. The initial analysis was done to make sure that the data made sense. For this, we plotted lumi signals and noise levels with relation to the beam current. Also, by using gain curves developed for every PMT in use, the amount of charge produced by the photocathode was estimated. When working properly, the noise should go down statistically like $\sigma \propto 1 / \sqrt{I_{\text {beam }}}$ as the beam current is increased. Figures B.4 and B.5 show data taken at several beam currents on a Carbon target. Overall, this data shows that the luminosity monitor signals behave as expected.

In order to determine a limit on the electronics noise, data was taken at a high beam current of $60 \mu \mathrm{A}$ with a thin lead target. PMT and ADC gains were set up to get a large signal ( $\approx 50000$ channels) in the 16-bit ADC range. This data, taken with the final configuration shown in Figure B.3 with and without shielding blocks, was used to set new limits on detector noise and to understand the contributions from a 

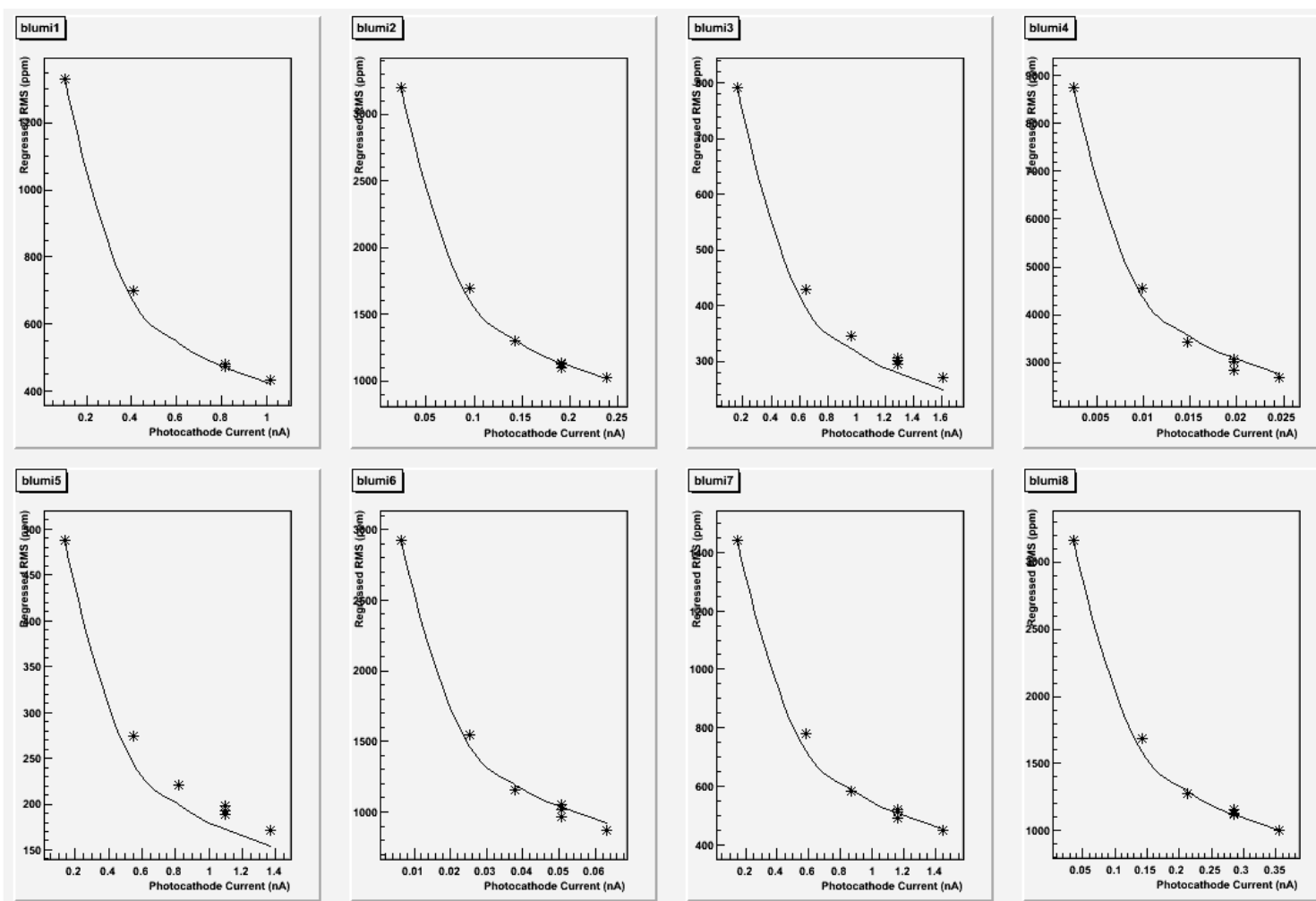

Figure B.5: Summary of noise levels for all the luminosity monitor data taken with carbon. The fits show how the RMS values (y-axis) should decrease as beam current goes up (x-axis).

variety of sources. Tables B.1 and B.2 show a summary of the analysis done for two specific runs, before and after shielding.

The analysis showed that, after taking into account noise from statistics, backgrounds, pedestals and correlations between signals, the noise levels were understood by assuming an extra contribution of about $100 \mathrm{ppm}$. The column labeled 'Blind/Unsh' was calculated by using ratios of measured current with respect to lumi4 before it was shielded (lumi4 had RMS $=442.2 \mathrm{ppm}$ before shielding). The column labeled 'Blind-Sh' was found the same way but by using lumi4 after shielding (lumi 4 had $\mathrm{RMS}=816.3 \mathrm{ppm})$.

Contributions from the BCM signal were found by studying the noise from the pedestal of the ADC channels used to mmeasure them. The pedestal noise contribution of about 20-30 ppm was the lowest such level ever measured using 16-bit ADCs. The correlated noise comes from an observed relationship between the pedestal noise 


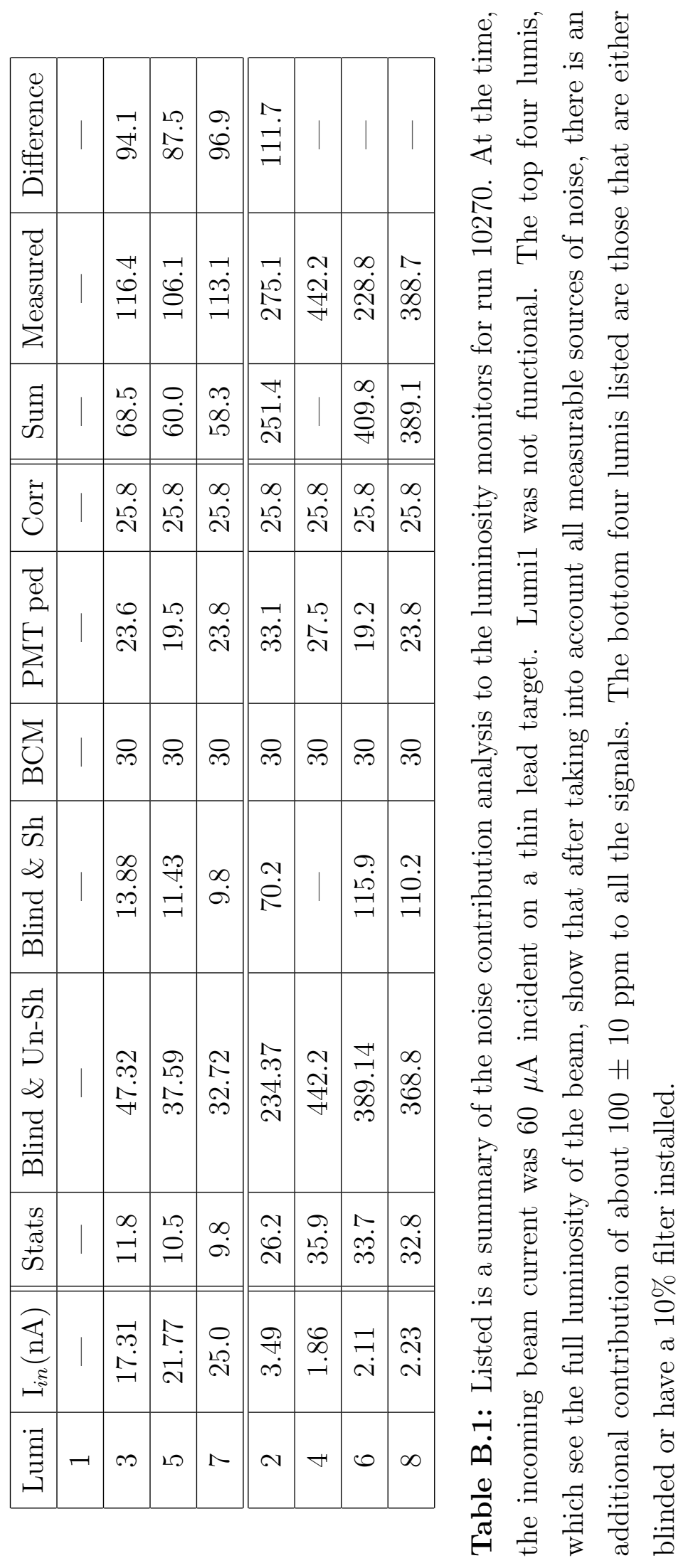




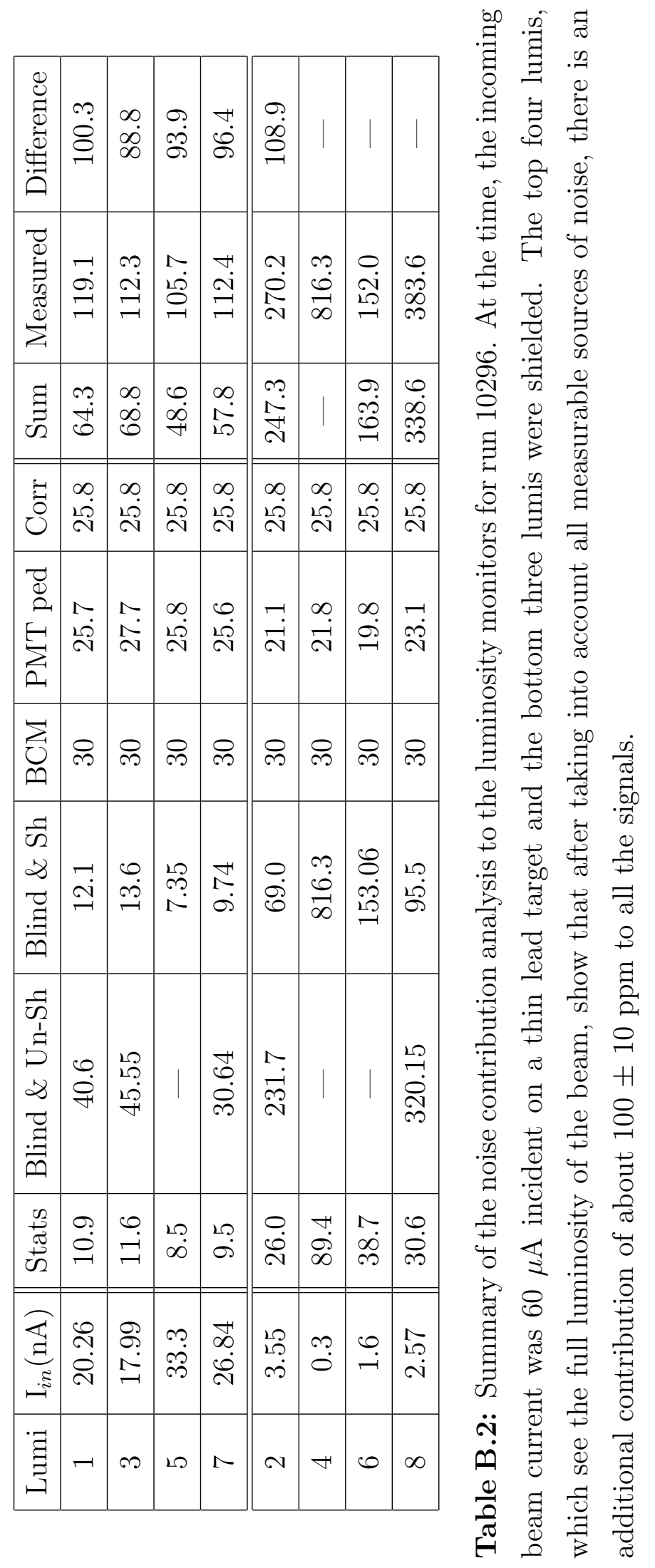




\begin{tabular}{|c|c|c|}
\hline Lumi \# & Asym RMS (ppm) & Cathode Current (nA) \\
\hline 1 & 119.1 & 20.26 \\
\hline 3 & 112.4 & 17.99 \\
\hline 5 & 105.7 & 33.3 \\
\hline 7 & 112.4 & 26.84 \\
\hline V & 55.7 & - \\
\hline H & 62.8 & - \\
\hline C & 51.7 & - \\
\hline \hline 2 & 270.2 & 3.55 \\
\hline 4 & 816.3 & 0.3 \\
\hline 6 & 152.0 & 1.6 \\
\hline 8 & 383.6 & 2.57 \\
\hline D1 & 149.6 & - \\
\hline D2 & 451.2 & - \\
\hline X & 241.1 & - \\
\hline SUM & 126.3 & - \\
\hline
\end{tabular}

Table B.3: Asymmetry RMS values for luminosity monitor signals. Data was taken with a $60 \mu \mathrm{A}$ beam current and a thin lead target. The values of photocathode current were estimated by using gain curves for all the PMTs used.

of different ADC channels. An essential result is that the sum of noise contributions for lumi8 is almost exactly the same as the measured RMS value for the unshielded data run.

By studying how the noise levels evolve when combining lumi signals, one can conclude that the extra source of noise is from position. As more lumis are combined, the noise should go down as $1 / \sqrt{N}$, where $N$ is the number of lumis. Table B.2 shows the RMS of pair-wise asymmetries measured with each luminosity monitor for run 10296. As expected, the lumis that have no ND filters see the highest signal with the lowest noise levels. Also listed are the RMS values for the combinations of lumis, were $\mathrm{H}$ is $1+5, \mathrm{~V}$ is $3+7, \mathrm{D} 1$ is $2+6, \mathrm{D} 2$ is $4+8, \mathrm{C}$ is $\mathrm{H}+\mathrm{V}, \mathrm{X}$ is $\mathrm{D} 1+\mathrm{D} 2$ and $\mathrm{SUM}$ is all eight lumis.

When the even numbered lumis are combined, any noise from position should 
vanish. This means that when lumi pairs are formed, the measured noise will decrease more than the $1 / \sqrt{N}$ expectation, which is what was observed in this data. By combining all four even lumis, the noise once again does not decrease as expected because we have reached the minimum noise level that can be measured with this experimental setup. This is what we refer to as the electronics noise floor level, which was measured to be about $50 \mathrm{ppm}$. This noise floor can be explained by adding in quadrature contributions from correlations and pedestals.

Since these tests took place, the luminosity monitors have been used by numerous experiments that have taken place in Hall A. They are a great tool for monitoring density fluctuations of both cryotargets and solid targets such as Carbon and Lead. The current configuration has no filters on any of the lumis. As will be discussed in Appendix D, the lumis were used to monitor for 'boiling' of the liquid hydrogen target. Such effects can add unwanted extra noise to pair-wise asymmetry measurements, increasing the required data to meet the experiment's error budget. 


\section{APPENDIX C}

\section{ENSURING QUALITY OF DATA WITH SYNC MONITOR}

\section{C.1 Synchronization Checks}

The data acquisition system reads out signals from several different electronics crates located all around the lab. While there are systems in place to make sure all the electronics are triggered and read out at the same time, redundant checks are desired. A synchronization monitor system that was developed for the second generation HAPPEx experiment by Bryan Moffit [57] was revived for this experiment, with some new features.

This system is designed to make sure that the integration gates controlled by the HAPPEx timing board in each crate are triggered simultaneously. To do this, two complimentary and pseudo-random frequency signals are sent to each crate, where they are recorded by the DAQ and analyzed to gain information about variations in start time between adjacent helicity windows.

The two synchronization signals were controlled by the MPS signal that triggers the helicity change at the polarized source. A pseudo-random variation was introduced to these signals using the digital-to-analog (DAC) output of the HAPPEx timing board in the Counting House crate. The MPS signal was routed to a gate generator, where the start of the gate was delayed so that it was in the middle of the $500 \mu \mathrm{s}$ 'settle time', as seen in Figures C.1 and C.2. The DAC signal was routed through a TRIUMF voltage-to-frequency converter and fanned out to a logic module. The GATE signal and its compliment $(\overline{\mathrm{GATE}})$ are also sent to the logic module, where the logical AND between them and the frequency signal is then integrated by a scaler channel in the HAPPEx DAQ. A schematic of this setup is shown in Figure C.3. 


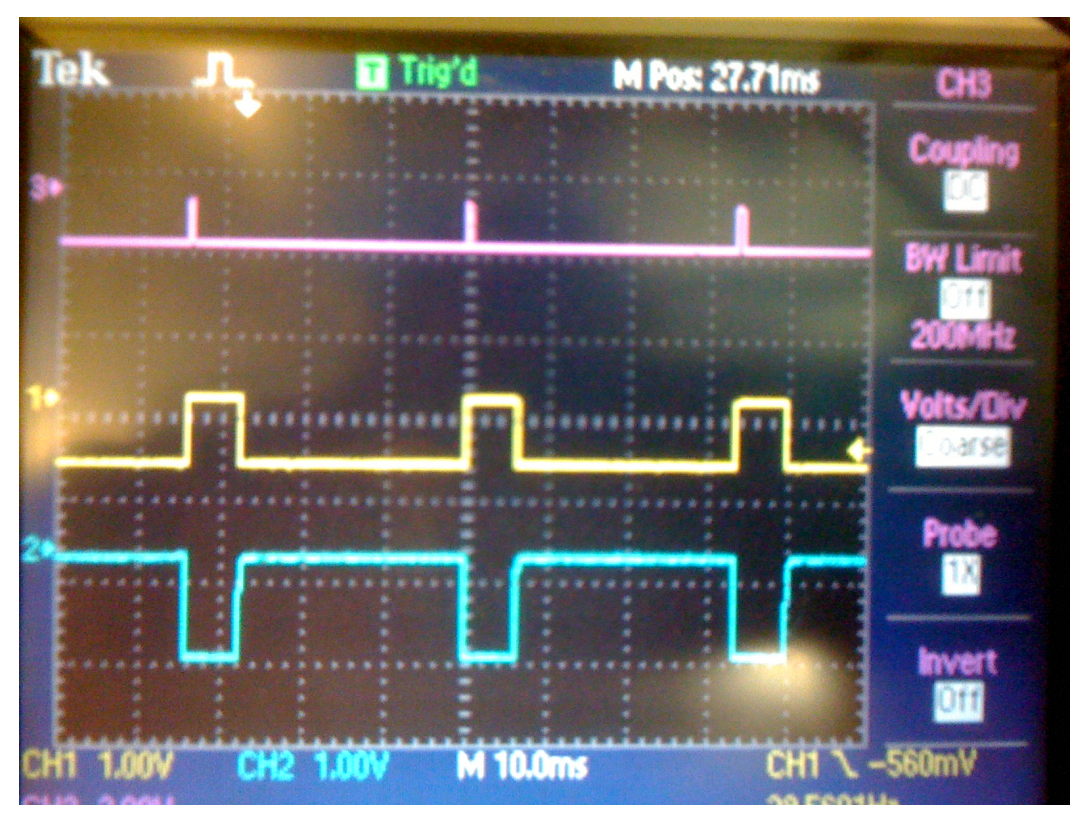

Figure C.1: Scope trace of main Synchronization Monitor signals. Shown are the MPS signal (pink), GATE (yellow) and its compliment (blue).

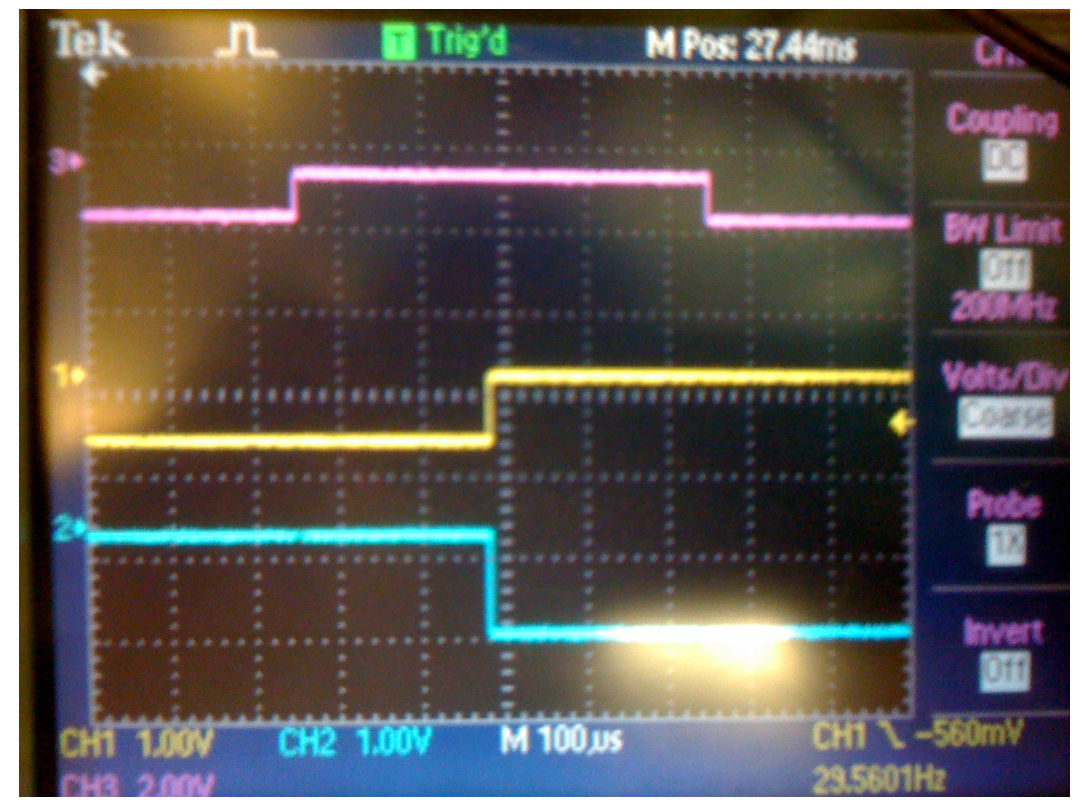

Figure C.2: Zoomed in version of Figure C.1. Note that the beginning of the gate starts in the middle of the MPS 'settle time'. 


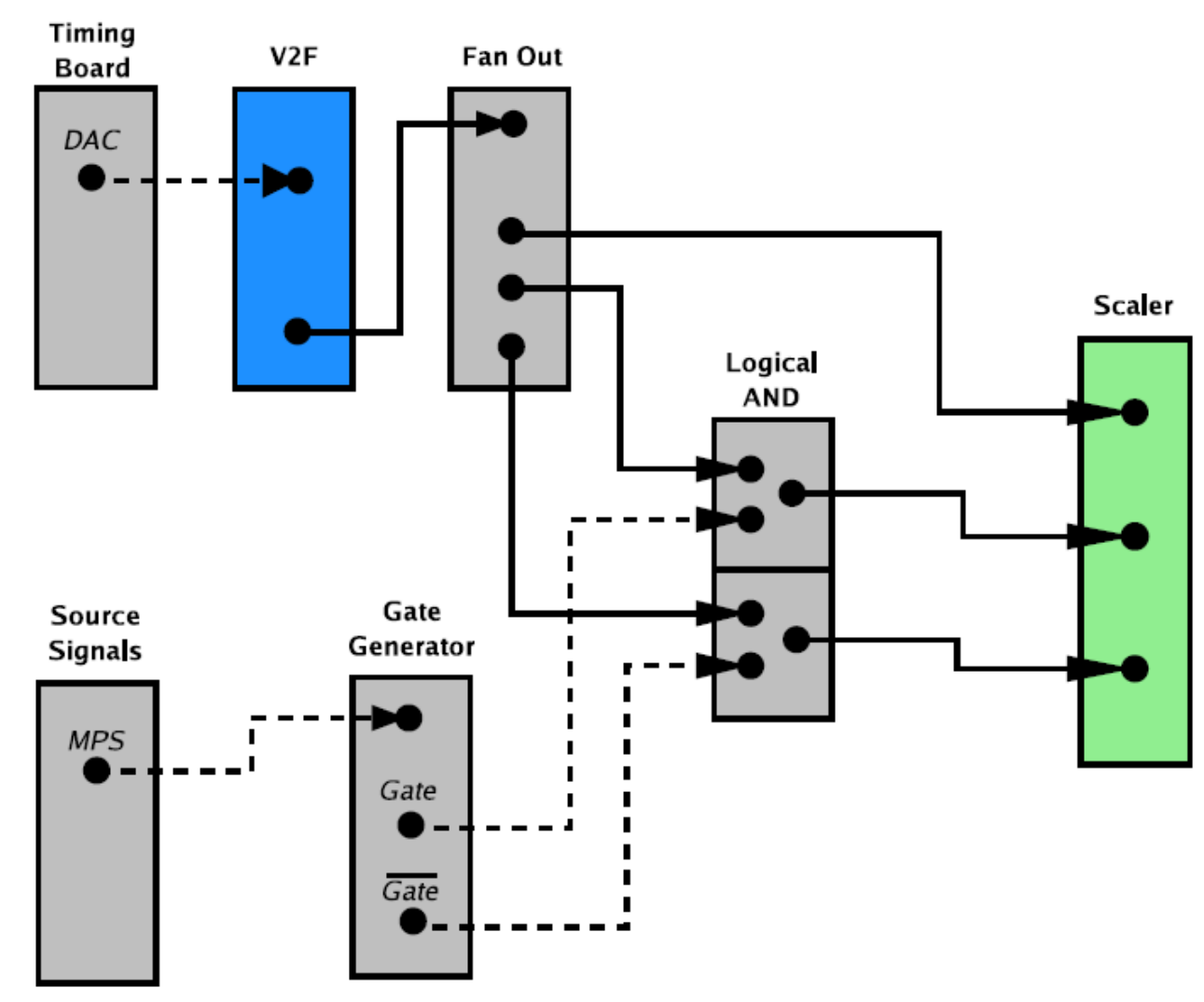

Figure C.3: Schematic of all the electronic elements used for the main Synchronization Monitor [57].

The gated frequency signals $f_{1}$ and $f_{2}$ were supplied to each electronics crate, located at the Counting House, Injector and the Left and Right Spectrometers. A third frequency signal $f_{r}$, un-gated by the logic module, served as a reference and was only connected to a scaler in the Counting House. It should be noted that the two signals received at the Injector were provided by an optical frequency output of the HAPPEx timing board and were sent via optical fibers. For this reason, the magnitude of the frequency signals at the Injector were not the same as for the other crates, but will still be proportional to them.

An extra check was added to the synchronization monitor for HAPPEx-III that did not rely on the scaler readouts. To do so, the same time-varying DAC signal was fanned out directly to a Voltage-mode 18-bit ADC channel in the Counting House and the Right Spectrometer DAQs. This was mainly done because we encountered some small readout problems with the Counting House scalers during the commisioning 
of the experiment. Since the reference frequency signal $f_{r}$ is only read in by these faulty scalers, this extra check provided extra confidence that the DAQ crates were synchronized throughout the entire experiment.

\section{C.1.1 Sync Monitor Analysis}

The most basic check to make sure that the signals in each crate are synchronized is to plot their correlation. The GATE and GATE signals at each electronics crate should be perfectly correlated with each other. Figure C.4 shows all such correlations for three of the electronics crates. The signals are all perfectly correlated, as is expected when there is perfect synchronization.

One can also figure out how good these correlation plots really are by plotting the difference of the signals divided by the reference signal, as seen in Figure C.5. As expected, these plots show some data at the value ' 1 ' and the rest converging at higher gating frequencies. Note that the plots should converge at '0' but an artificial offset has been added for plotting purposes.

Figure C.6 shows some more detail about the correlation check between the GATE signals at the Right Spectrometer and the Counting House crates. The top left plot shows what the GATE signal looks like for the Right Spectrometer with respect to time. This shows that the signal is indeed random through a certain range. The top right and bottom left plots show the correlation at each crate of the GATE signal and the DAC signal provided by the Counting House timing board. Finally, the bottom right plot shows the effective synchronization for the whole run. For perfect synchronization, all the events should be at zero. The events seen at \pm 1 occur from single missed frequency pulses that lie too close to the beginning or end of the integration gate. This is the same data that is shown converging in Figure C.5 These are expected and do not imply bad synchronization.

During the experiment, alerts were created using online data analysis that would let shift workers know that there was a synchronization problem. Figure C.7 shows one of the plots that the shift crew had to check throughout the experiment. If these plots became anything other than a clear linear correlation, a DAQ expert was called to check the source of the problem and fix it. 


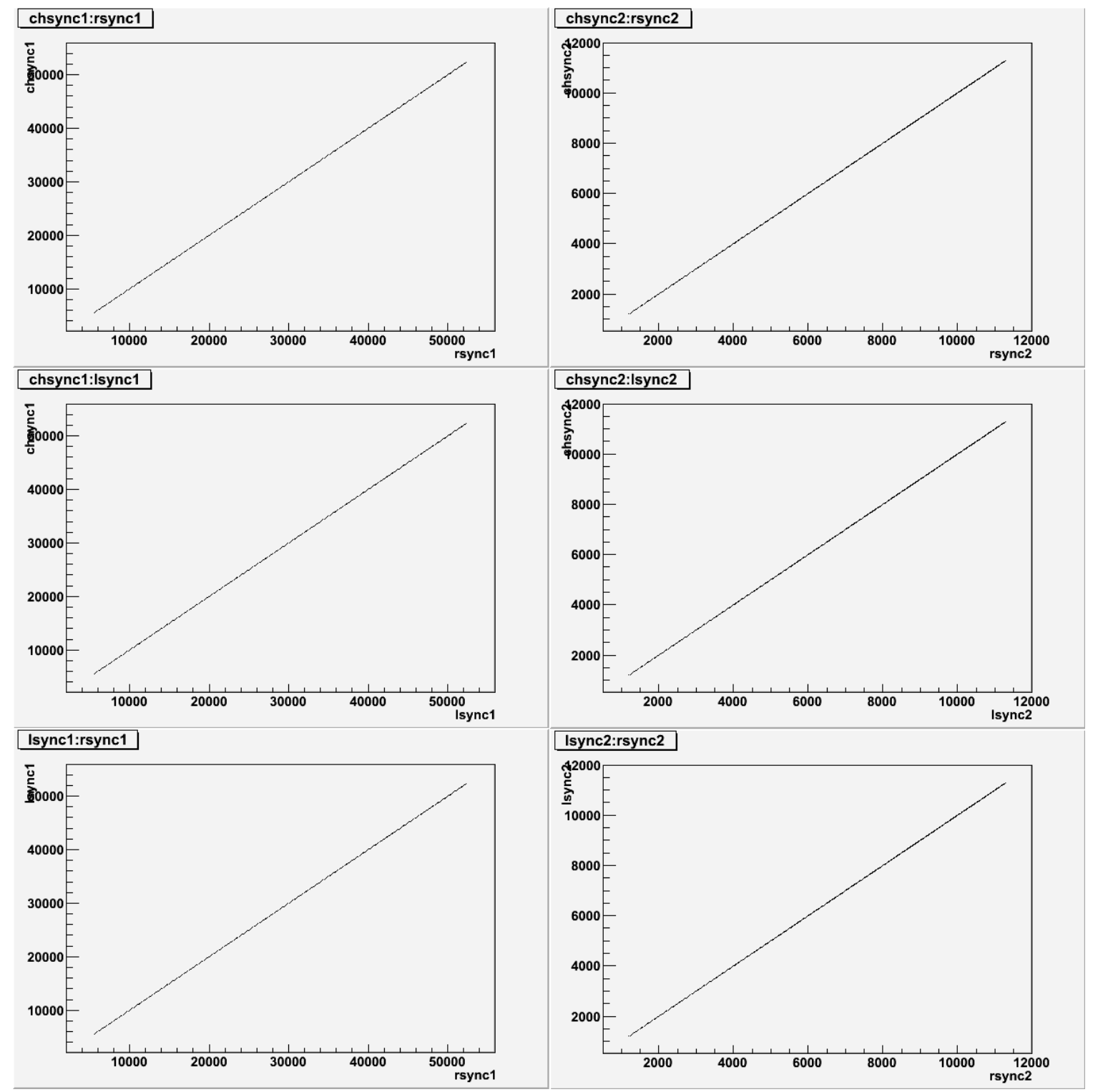

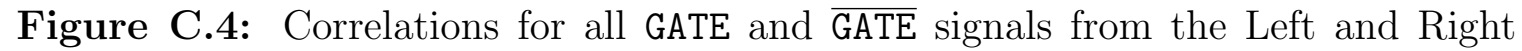
Spectrometers and the Counting House DAQ. The column on the left shows all the GATE correlations. The column on the right shows all the GATE correlations. 


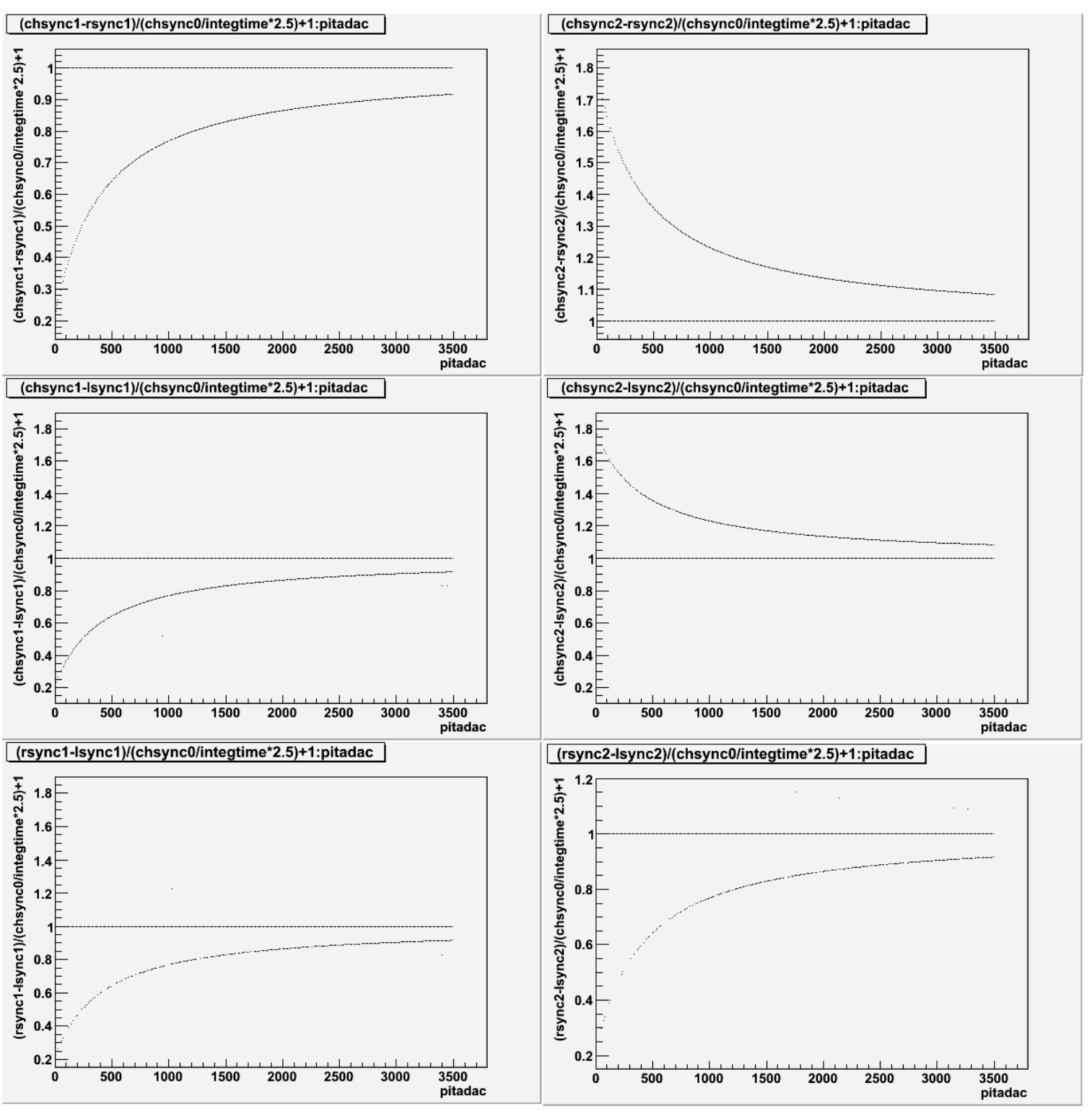

Figure C.5: This set of plots shows another method of making sure all the electronics are synchronized, by studying how well correlated the plots in Figure C.4 really are. As expected, most of the data is at ' 1 ' and the rest converges as the frequency signal is increased. 

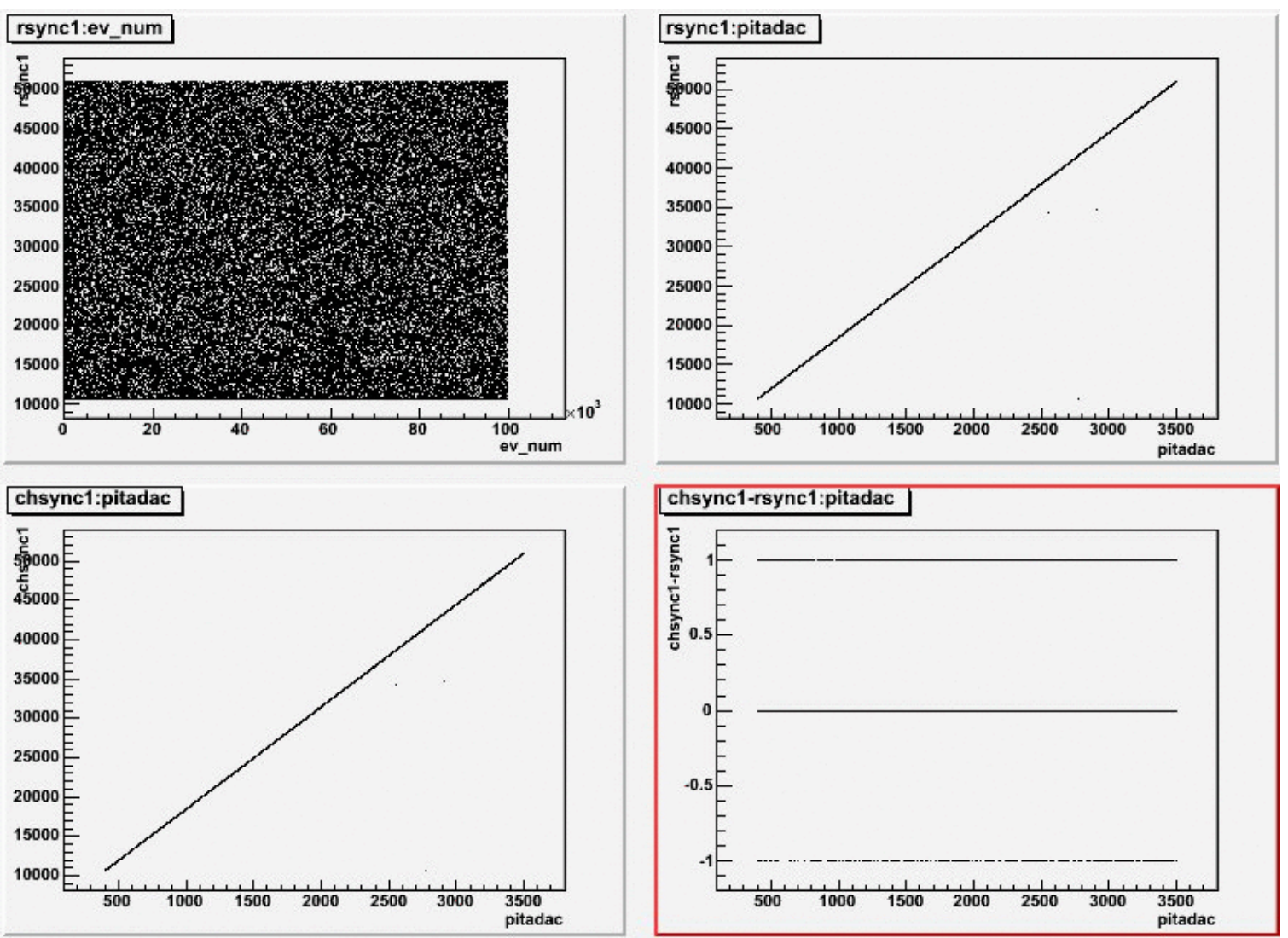

Figure C.6: Sample set of plots used to determine the synchronization between the Right Spectrometer and the Counting House crates. 


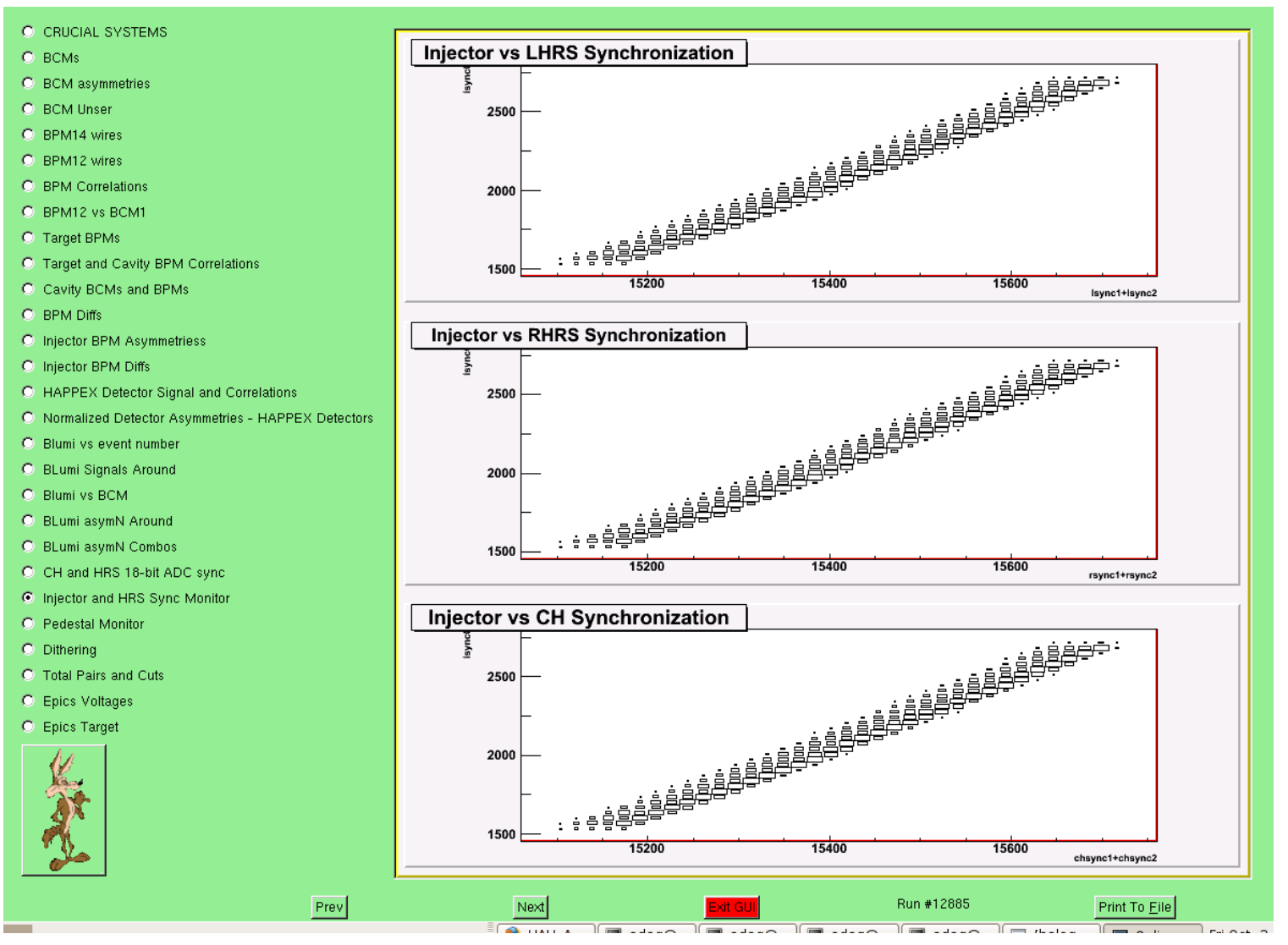

Figure C.7: Example of plots monitored by the shift crew to make sure synchronization between the electronics crates was good. 


\section{APPENDIX D}

\section{MINIMIZING BOILING EFFECTS WITH CRYOTARGETS}

The liquid hydrogen cryotargets used in the HAPPEx-III experiment can suffer from beam induced density fluctuations, leading to an effect referred to as 'target boiling.' By using the luminosity monitors installed downstream of the target, such effects can be studied and minimized by making adjustments to such things as the raster size and fan speed.

During the commissioning period of the experiment, tests on the $25 \mathrm{~cm}$ liquid hydrogen cryotarget were carried out in which beam current, raster size and fan speed were varied incrementally to determine the best conditions for running with minimal target boiling effects. Such effects are manifested as the onset of non-statistical fluctuations in the width of the detector's pair-wise asymmetry measurement. Any small changes in the target density between adjacent helicity windows will cause fluctuations in the scattering rate that degrade the asymmetry beyond Poisson counting statistics.

For HAPPEx-III the expected counting rate for each detector was about $1.1 \mathrm{MHz}$ at a beam current of $100 \mu \mathrm{A}$. This means that the statistical width of the asymmetry distribution for each detector, after taking radiative corrections into effect, should be about 5000 ppm (parts per million). This can be seen in Figure D.1, where the red curve represents how the detector noise should decrease with increasing current, according to Poisson counting statistics

$$
\sigma_{\text {det }} \propto 1 / \sqrt{I_{\text {beam }}}
$$

The blue data curve is the noise measured with Detector 1 at several beam currents. The difference between the two curves can be accounted for by the extra fluctuations caused by target boiling. 


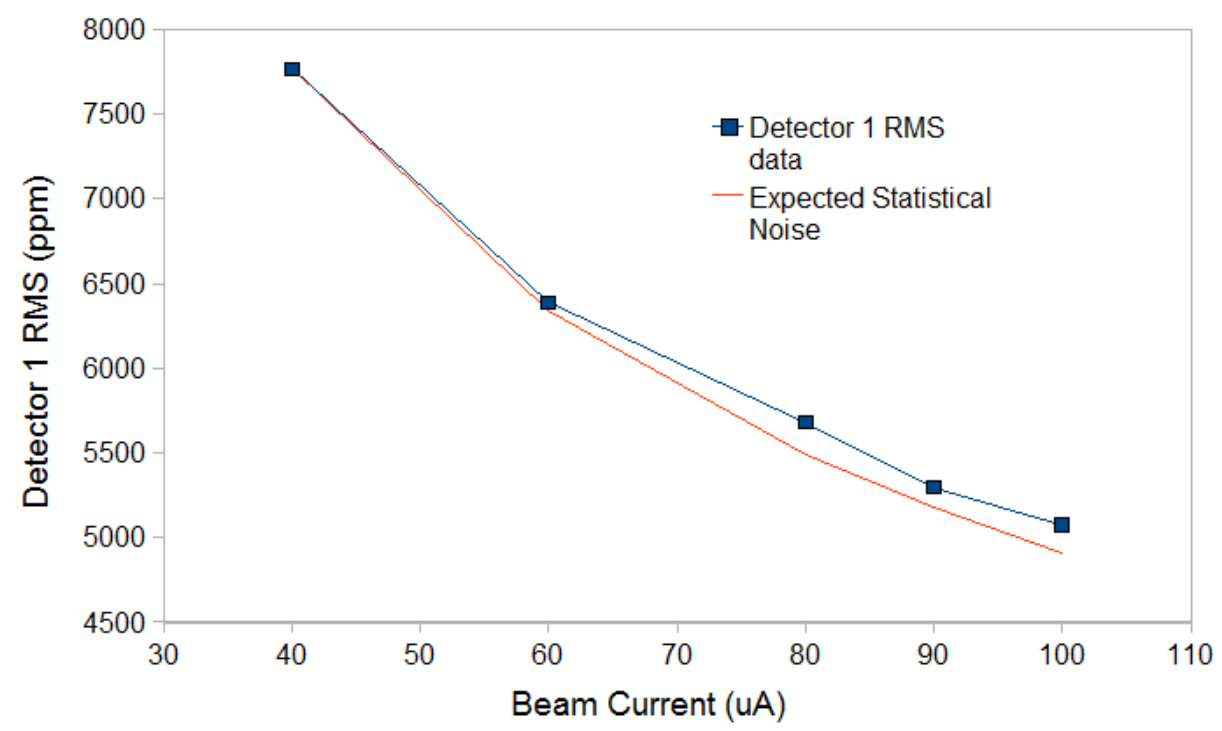

Figure D.1: RMS width of asymmetries as measured by Detector 1, located in the L-HRS. The blue curve represents data taken at several different currents. The red curve is the expected statistical width according to Poisson statistics. The difference in these curves is caused by target 'boiling' fluctuations.

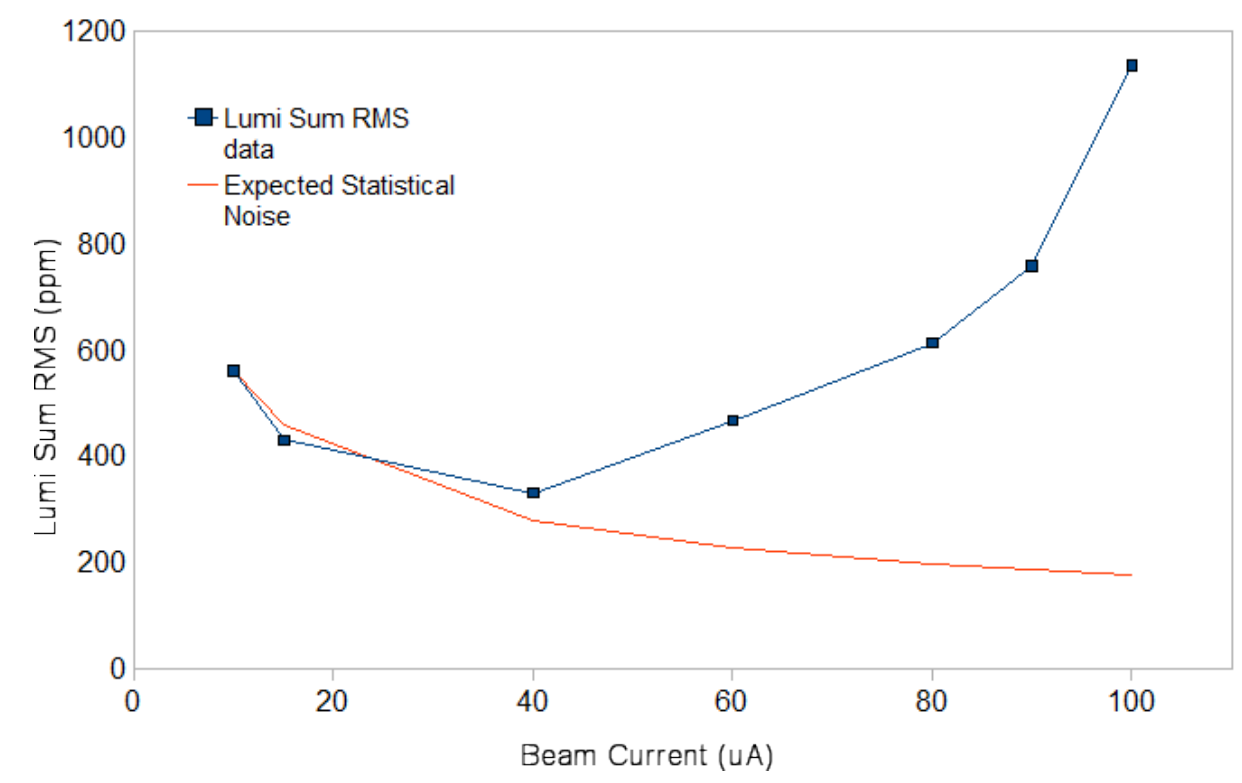

Figure D.2: RMS width of the sum of all eight lumi signals. Assuming dominance of statistics at $10 \mathrm{nA}$, data points at very low current behave properly (red curve), but at higher currents, target density fluctuations add non-statistical noise to the distribution. 


\begin{tabular}{|c|c|c|}
\hline Fan Speed $(\mathrm{Hz})$ & Detector1 RMS $(\mathrm{ppm})$ & Lumi Sum RMS $(\mathrm{ppm})$ \\
\hline 63 & 6448 & 875 \\
\hline 68 & 6470 & 790 \\
\hline 72 & 6437 & 676 \\
\hline
\end{tabular}

Table D.1: Summary of fan speed boiling test. The data shows that the effects of the fan speed on the detector widths is barely noticeable. For this reason, HAPPEx-III ran with a fan speed of $72 \mathrm{~Hz}$

Figure D.2 shows the usefulness of the Luminosity Monitor in measuring such contributions. The data shown is the noise of the combination of all eight luminosity monitors. Using the 'lumi sum' helps get rid of systematic errors that might depend on the position of each individual lumi. The trend seen in this plot shows where the target boiling fluctuations begin to be a problem; approximately at beam currents higher than $\approx 40 \mu \mathrm{A}$. At this point, the noise of the lumi sum asymmetry distribution blows up in a non-statistical way. By subtracting this noise in quadrature from the measured detector noise, we obtain values very close to the expected statistical noise, proving the accuracy of this technique.

The results of this test showed that at $100 \mu \mathrm{A}$, target boiling contributes about $1300 \mathrm{ppm}$ of extra noise to the detector asymmetries. This contribution adds $2.8 \%$ of noise to the detector distribution. If the boiling noise were to get any worse than $2000 \mathrm{ppm}$, the noise would get worse by about $8 \%$.

During the experiment's data taking period, DAQ alarms were created to let shift crews know when the lumi widths got worse than $1200 \mathrm{ppm}$. At least one time during the run, the lumi sum noise became about $2500 \mathrm{ppm}$, which would degrade the asymmetry distributions by $12 \%$. If unaccounted for, this effect would have increased the necessary data taking period by $24 \%$ in order to achieve the proposed precision.

Two other tests were conducted to minimize target density fluctuations. For one, data was taken at three different fan speeds at a beam current of $60 \mu \mathrm{A}$. For the other test, conducted at $70 \mu \mathrm{A}$ and a fan speed oof $72 \mathrm{~Hz}$, we studied the fluctuations caused by using different raster sizes. The results for the fan speed test are summarized in Table D.1. We found that the fluctuations did not vary much with different fan speeds. This was not the case for different raster sizes. 


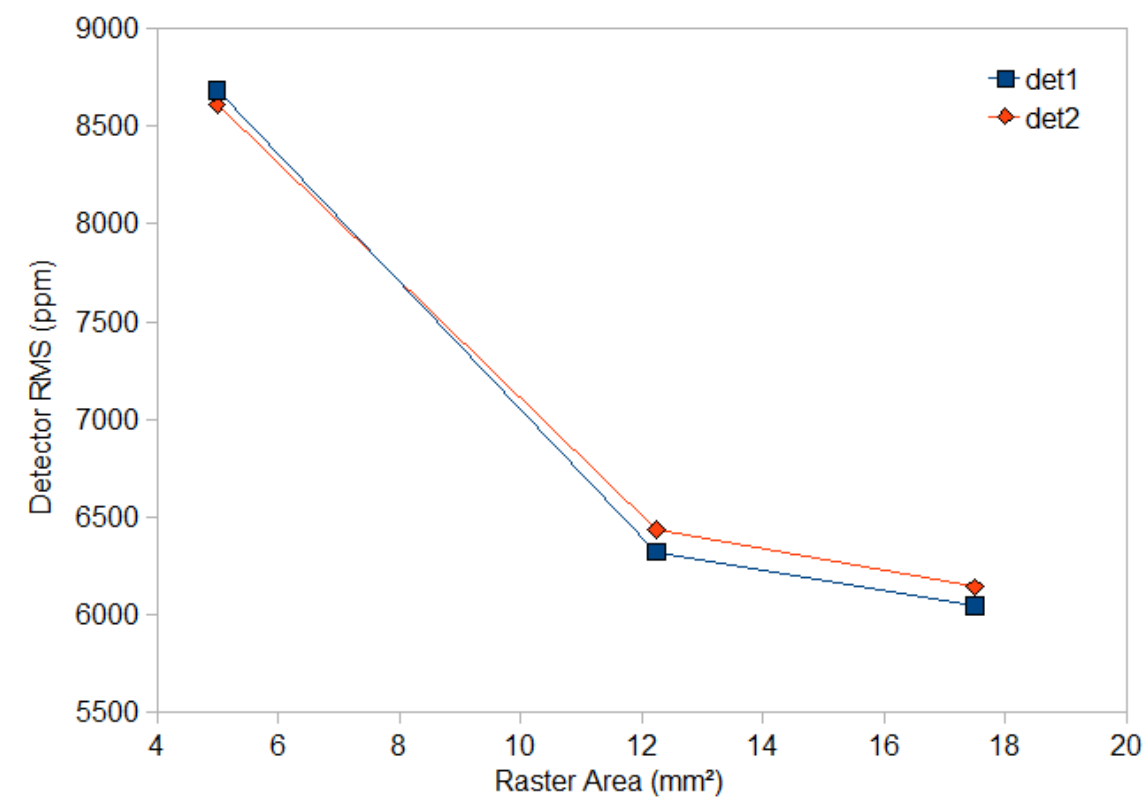

Figure D.3: RMS width of asymmetries as measured by both detectors with different raster sizes while running at $70 \mu \mathrm{A}$ with a fan speed of $72 \mathrm{~Hz}$.

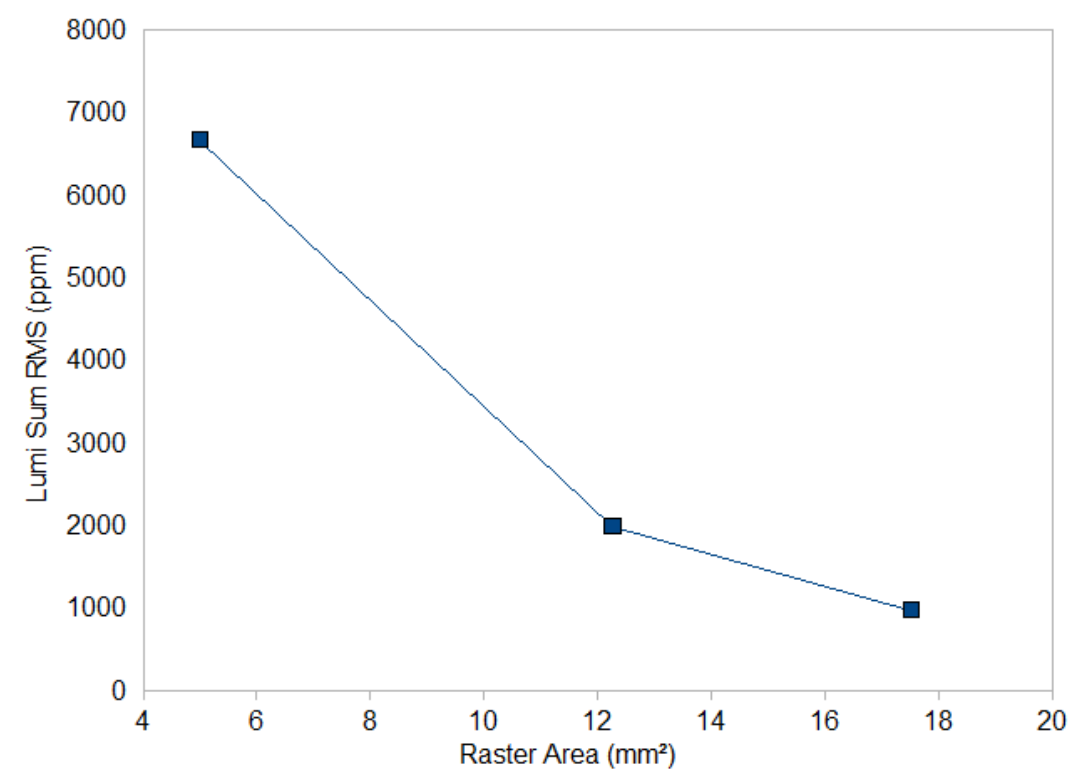

Figure D.4: RMS width of the sum of all eight lumi monitor signals with different raster sizes while running at $70 \mu \mathrm{A}$ with a fan speed of $72 \mathrm{~Hz}$. 
The data plotted in Figures D.3 and D.4 show a very strong dependence of target density fluctuations with the raster size. The raster sizes used for this study were $2.0 \times 2.5,3.5 \times 3.5$ and $3.5 \times 5.0 \mathrm{~mm}$ and correspond accordingly to the three areas used in the plot. As the area of the raster increases, the fluctuations decrease dramatically. For this reason, HAPPEx-III ran with the $3.5 \times 5.0 \mathrm{~mm}$ raster. In fact, this was also the raster size used for the current ramp boiling test summarized at the beginning of this section. 


\section{REFERENCES}

[1] B. Pohv et al., Particles and Nuclei: An Introduction to the Physical Concepts (2006).

[2] R. Hofstadter. The electron-scattering method and its application to the structure of nuclei and nucleons. Nobel Lectures (1964).

[3] AESCIMED/Science Photo Library. Visualization of quark structure of carbon. http://www.sciencephoto.com/media/2108/view\#.

[4] E. Noether. Invariiante Variationsprobleme. Nachr. v. d. Ges. d. Wiss. zu Gottingen, 235-257 (1918).

[5] T. D. Lee and C. N. Yang. Question of Parity Conservation in Weak interactions. Phys. Rev. 104, 254 (1956).

[6] J. Ashman et al. [European Muon Collaboration], Phys. Lett. B 206, 364 (1988).

[7] D. B. Kaplan and A. Manohar, Nucl. Phys. B310, 527 (1988).

[8] M. N. Rosenbluth. Phys. Rev. D, 79:615, 1950.

[9] T. W. Donnelly, J. Dubach and Ingo Sick, Nuc. Phys. A 503 (1989) 589.

[10] C. J. Horowitz and J. Piekarewicz, Phys. Rev. C 64, (2001) 062802.

[11] C. J. Horowitz and J. Piekarewicz, Phys. Rev. Lett. 86, 5647 (2001).

[12] P. Ring et al., Nucl. Phys. A 624, 349 (1997).

[13] R. J. Furnstahl, Nucl. Phys. A 706, 85 (2002).

[14] K. S. Kumar and P. A. Souder. Strange Quarks and Parity Violation. Prog. Part. Nucl. Phys., 45:S333-S395, 2000.

[15] J. Alcorn, et al., Nucl. Instrum. Meth. A522, 294-346 (2004).

[16] K. D. Paschke, Eur. Phys. J. A32, 549-553 (2007).

[17] T. Maruyama et al., Appl. Phys. Lett. 85, 2640 (2004).

[18] W. Barry et al., Beam position measurement in the CEBAF recirculating linacs by use of pseudorandom pulse sequences. JLab-TN-90-246 (1990).

[19] K. Unser, IEEE Trans. Nucl. Sci. NS 28 (1981).

[20] A. Acha, et al., Phys. Rev. Lett. 98, (2007) 032301.

[21] K. Nakamura et al., J. Phys. G 37, (2010) 075021.

[22] D. Meekins, Hall A Target Configuration: HAPPEx-III and PVDIS. Unpublished tech note. (2009).

[23] R. Silwal, Ph. D. Thesis, University of Virginia, 2012. 
[24] Grady Wilson Miller IV, Ph.D. thesis, Princeton University, 2001, Parityviolation in Forward Angle Elastic Electron-proton Scattering.

[25] B. Hahn et al., Tests of the PREX 18-bit ADCs. Unpublished tech note. (2008).

[26] M. Friend et al., Upgraded photon calorimeter with integrating readout for Hall A Compton Polarimeter at Jefferson Lab (2011).

[27] D. W. Higinbotham, AIP Conf. Proc. 1149, 751754 (2009), 0901.4484.

[28] R. Michaels, D. Meekins and P. Adderly, Target Info for PREX. Unpublished tech note. 062310.

[29] D. McNulty, Results from Jan2008 PREx Detector Beamtest. Unpublished tech note. (2008).

[30] Rene Brun and Fons Rademakers, ROOT - An Object Oriented Data Analysis Framework, Proceedings AIHENP'96 Workshop, Lausanne, Sep. 1996, Nucl. Inst. \& Meth. in Phys. Res. A 389 (1997) 81-86. See also http://root.cern.ch/.

[31] L.W. Whitlow et al., Phys. Lett. B 282 (1992) 475; Phys. Lett. B 250 (1990) 193.

[32] M.E. Christy, P.E. Bosted, Phys.Rev.C 81, 055213 (2010).

[33] N. Falletto et al. Compton Scattering off polarized electron with a high-finesse Fabry-Perot Cavity in Jab. Nucl. Inst. \& Meth. in Phys. Res. A 459 412-425 (2001).

[34] D. Parno et al. Preliminary Results from Integrating Compton Photon Polarimetry in Hall A of Jefferson Lab. (2011).

[35] K. Paschke et al., HAPPEX III Qsq Summary. (2011) 012811.

[36] M.J. Musolf et al., Phys. Rep., 239:1, (1994).

[37] T.W. Donnelly and M.J. Musolf. Nucl. Phys. A, 550:509, (1992).

[38] J. Arrington, I. Sick, Phys. Rev. Lett. C76, (2007) 035201.

[39] E. J. Beise, M. L. Pitt, D. T. Spayde, Prog. in Part. and Nucl. Phys. 54 (2004).

[40] D. T. Spayde et al., Phys. Lett. B 583 (2004) 79.

[41] F. E. Maas et al., Phys. Rev. Lett. 93 (2004) 022002.

[42] F. E. Maas et al., Phys. Rev. Lett. 94 (2005) 152001.

[43] S. Baunack et al., Phys. Rev. Lett. 102 (2009) 151803.

[44] K. A. Aniol et al., Phys. Lett. C 69 (2004) 065501.

[45] A. Acha et al., Phys. Lett. 98032301 (2007).

[46] D. S. Armstrong et al., Phys. Rev. Lett. 95 (2005) 092001.

[47] D. Androic et al., Phys. Rev. Lett. 104 (2010) 012001. 
[48] J. Liu, R. D. McKeown, and M. J. Ramsey-Musolf, Phys. Rev. C 76, 025202 (2007).

[49] D. Leinweber et al., Phys. Rev. Lett. 94, 212001 (2006); D . Leinweber et al., Phys. Rev. Lett. 97, 022001 (2006); P. Wang et al., Phys. Rev. C 79, 065202 (2009).

[50] T. Doi et al., Phys. Rev. D 80, 094503 (2009).

[51] R. D. Young et al., Phys. Rev. Lett. 97, 102002 (2006).

[52] R. D. Young et al., Phys. Rev. Lett. 99, 122003 (2007).

[53] S. -L. Zhu, S. J. Puglia, B. R. Holstein, M. J. Ramsey-Musolf, Phys. Rev. Lett. D62, 033008 (2000).

[54] R. Lewis et al., Phys. Rev. D 67, 013003 (2003).

[55] P. A. Souder et al., Prex-II: Precision Parity-Violating Measurement of the Neutron Skin of Lead. (2011).

[56] S. Baunack, Parity violating electron scattering. Talk given at 'Meson Production at Intermediate and High Energies' International Workshop (2011).

[57] Bryan Moffit, Ph.D. thesis, College of William and Mary, 2004, Elastic Scattering of Longitudinally Polarized Electrons from ${ }^{4} H e$ : A measurement of $G_{E}^{s}$ at $Q^{2}=0.1(\mathrm{GeV} / \mathrm{c})^{2}$. 